\title{
Cochrane
}

Library

Cochrane Database of Systematic Reviews

\section{Preventive lipid-based nutrient supplements given with complementary foods to infants and young children 6 to 23 months of age for health, nutrition, and developmental outcomes (Review)}

Das JK, Salam RA, Hadi YB, Sadiq Sheikh S, Bhutta AZ, Weise Prinzo Z, Bhutta ZA

Das JK, Salam RA, Hadi YB, Sadiq Sheikh S, Bhutta AZ, Weise Prinzo Z, Bhutta ZA.

Preventive lipid-based nutrient supplements given with complementary foods to infants and young children 6 to 23 months of age for health, nutrition, and developmental outcomes.

Cochrane Database of Systematic Reviews 2019, Issue 5. Art. No.: CD012611.

DOI: 10.1002/14651858.CD012611.pub3.

www.cochranelibrary.com

Preventive lipid-based nutrient supplements given with complementary foods to infants and young children 6 to 23 months of age for health, nutrition, and developmental outcomes (Review)

Copyright @ 2019 The Cochrane Collaboration. Published by John Wiley \& Sons, Ltd. 
TABLE OF CONTENTS

HEADER

ABSTRACT

PLAIN LANGUAGE SUMMARY

SUMMARY OF FINDINGS

BACKGROUND

OBJECTIVES

METHODS

RESULTS

Figure 1.

Figure 2.

Figure 3.

Figure 4.

Figure 5.

DISCUSSION

AUTHORS' CONCLUSIONS

ACKNOWLEDGEMENTS

REFERENCES

CHARACTERISTICS OF STUDIES

DATA AND ANALYSES

Analysis 1.1. Comparison 1 LNS versus no intervention, Outcome 1 Moderate stunting.

Analysis 1.2. Comparison 1 LNS versus no intervention, Outcome 2 Severe stunting.

Analysis 1.3. Comparison 1 LNS versus no intervention, Outcome 3 Moderate stunting: Sensitivity analysis.

Analysis 1.4. Comparison 1 LNS versus no intervention, Outcome 4 Severe stunting: Sensitivity analysis.

Analysis 1.5. Comparison 1 LNS versus no intervention, Outcome 5 Moderate wasting.

Analysis 1.6. Comparison 1 LNS versus no intervention, Outcome 6 Severe wasting.

Analysis 1.7. Comparison 1 LNS versus no intervention, Outcome 7 Moderate wasting: Sensitivity analysis.

Analysis 1.8. Comparison 1 LNS versus no intervention, Outcome 8 Severe wasting: Sensitivity analysis.

Analysis 1.9. Comparison 1 LNS versus no intervention, Outcome 9 Moderate underweight.

Analysis 1.10. Comparison 1 LNS versus no intervention, Outcome 10 Severe underweight.

Analysis 1.11. Comparison 1 LNS versus no intervention, Outcome 11 Moderate underweight: Sensitivity analysis. ..................

Analysis 1.12. Comparison 1 LNS versus no intervention, Outcome 12 Severe underweight: Sensitivity analysis.

Analysis 1.13. Comparison 1 LNS versus no intervention, Outcome 13 Anaemia.

Analysis 1.14. Comparison 1 LNS versus no intervention, Outcome 14 Adverse effects.

Analysis 1.15. Comparison 1 LNS versus no intervention, Outcome 15 Adverse effects: Sensitivity analysis.

Analysis 1.16. Comparison 1 LNS versus no intervention, Outcome 16 Mid-upper arm circumference (MUAC).

Analysis 1.17. Comparison 1 LNS versus no intervention, Outcome 17 Serum haemoglobin (g/L).

Analysis 1.18. Comparison 1 LNS versus no intervention, Outcome 18 Mortality.

Analysis 1.19. Comparison 1 LNS versus no intervention, Outcome $19 \mathrm{HAZ}$.

Analysis 1.20. Comparison 1 LNS versus no intervention, Outcome 20 WAZ.

Analysis 1.21. Comparison 1 LNS versus no intervention, Outcome $21 \mathrm{WHZ}$.

Analysis 2.1. Comparison 2 LNS versus no intervention: Subgroup analysis by energy content/formulation of product provided, Outcome 1 Severe stunting.

Analysis 2.2. Comparison 2 LNS versus no intervention: Subgroup analysis by energy content/formulation of product provided, Outcome 2 Moderate stunting.

Analysis 2.3. Comparison 2 LNS versus no intervention: Subgroup analysis by energy content/formulation of product provided, Outcome 3 Moderate wasting.

Analysis 2.4. Comparison 2 LNS versus no intervention: Subgroup analysis by energy content/formulation of product provided, Outcome 4 Severe wasting.

Analysis 2.5. Comparison 2 LNS versus no intervention: Subgroup analysis by energy content/formulation of product provided, Outcome 5 Moderate underweight.

Analysis 2.6. Comparison 2 LNS versus no intervention: Subgroup analysis by energy content/formulation of product provided, Outcome 6 Severe underweight.

Preventive lipid-based nutrient supplements given with complementary foods to infants and young children 6 to 23 months of age for health, nutrition, and developmental outcomes (Review)

Copyright $\odot 2019$ The Cochrane Collaboration. Published by John Wiley \& Sons, Ltd. 
Analysis 2.7. Comparison 2 LNS versus no intervention: Subgroup analysis by energy content/formulation of product provided, Outcome 7 Anaemia.

Analysis 2.8. Comparison 2 LNS versus no intervention: Subgroup analysis by energy content/formulation of product provided, Outcome 8 Adverse effects.

Analysis 2.9. Comparison 2 LNS versus no intervention: Subgroup analysis by energy content/formulation of product provided, Outcome 9 Mid-upper arm circumference (MUAC).

Analysis 2.10. Comparison 2 LNS versus no intervention: Subgroup analysis by energy content/formulation of product provided, Outcome 10 Serum haemoglobin $(\mathrm{g} / \mathrm{L})$.

Analysis 2.11. Comparison 2 LNS versus no intervention: Subgroup analysis by energy content/formulation of product provided, Outcome 11 Mortality.

Analysis 2.12. Comparison 2 LNS versus no intervention: Subgroup analysis by energy content/formulation of product provided, Outcome $12 \mathrm{HAZ}$.

Analysis 2.13. Comparison 2 LNS versus no intervention: Subgroup analysis by energy content/formulation of product provided, Outcome $13 \mathrm{WHZ}$.

Analysis 2.14. Comparison 2 LNS versus no intervention: Subgroup analysis by energy content/formulation of product provided, Outcome 14 WAZ.

Analysis 3.1. Comparison 3 LNS versus no intervention: Subgroup analysis by duration of intervention, Outcome 1 Moderate stunting.

Analysis 3.2. Comparison 3 LNS versus no intervention: Subgroup analysis by duration of intervention, Outcome 2 Severe stunting.

Analysis 3.3. Comparison 3 LNS versus no intervention: Subgroup analysis by duration of intervention, Outcome 3 Moderate wasting.

Analysis 3.4. Comparison 3 LNS versus no intervention: Subgroup analysis by duration of intervention, Outcome 4 Severe wasting: 6 to 12 months.

Analysis 3.5. Comparison 3 LNS versus no intervention: Subgroup analysis by duration of intervention, Outcome 5 Moderate underweight.

Analysis 3.6. Comparison 3 LNS versus no intervention: Subgroup analysis by duration of intervention, Outcome 6 Severe underweight: 6 to 12 months.

Analysis 3.7. Comparison 3 LNS versus no intervention: Subgroup analysis by duration of intervention, Outcome 7 Anaemia. .. Analysis 3.8. Comparison 3 LNS versus no intervention: Subgroup analysis by duration of intervention, Outcome 8 Adverse effects.

Analysis 3.9. Comparison 3 LNS versus no intervention: Subgroup analysis by duration of intervention, Outcome 9 Mid-upper arm circumference (MUAC).

Analysis 3.10. Comparison 3 LNS versus no intervention: Subgroup analysis by duration of intervention, Outcome 10 Serum haemoglobin (g/L).

Analysis 3.11. Comparison 3 LNS versus no intervention: Subgroup analysis by duration of intervention, Outcome 11 Mortality.

Analysis 3.12. Comparison 3 LNS versus no intervention: Subgroup analysis by duration of intervention, Outcome 12 HAZ. .... Analysis 3.13. Comparison 3 LNS versus no intervention: Subgroup analysis by duration of intervention, Outcome 13 WAZ. .... Analysis 3.14. Comparison 3 LNS versus no intervention: Subgroup analysis by duration of intervention, Outcome 14 WHZ. ... Analysis 4.1. Comparison 4 LNS versus no intervention: Subgroup analysis by age at follow-up, Outcome 1 Moderate stunting. . Analysis 4.2. Comparison 4 LNS versus no intervention: Subgroup analysis by age at follow-up, Outcome 2 Severe stunting. ... Analysis 4.3. Comparison 4 LNS versus no intervention: Subgroup analysis by age at follow-up, Outcome 3 Moderate wasting. . Analysis 4.4. Comparison 4 LNS versus no intervention: Subgroup analysis by age at follow-up, Outcome 4 Severe wasting. ... Analysis 4.5. Comparison 4 LNS versus no intervention: Subgroup analysis by age at follow-up, Outcome 5 Moderate underweight.

Analysis 4.6. Comparison 4 LNS versus no intervention: Subgroup analysis by age at follow-up, Outcome 6 Severe underweight: at 18 months.

Analysis 4.7. Comparison 4 LNS versus no intervention: Subgroup analysis by age at follow-up, Outcome 7 Anaemia. ............ Analysis 4.8. Comparison 4 LNS versus no intervention: Subgroup analysis by age at follow-up, Outcome 8 Adverse effects: At 18 months.

Analysis 4.9. Comparison 4 LNS versus no intervention: Subgroup analysis by age at follow-up, Outcome 9 Mid-upper arm circumference (MUAC).

Analysis 4.10. Comparison 4 LNS versus no intervention: Subgroup analysis by age at follow-up, Outcome 10 Serum haemoglobin (g/L).

Analysis 4.11. Comparison 4 LNS versus no intervention: Subgroup analysis by age at follow-up, Outcome 11 Mortality. ......... 
Analysis 4.12. Comparison 4 LNS versus no intervention: Subgroup analysis by age at follow-up, Outcome 12 HAZ.

Analysis 4.13. Comparison 4 LNS versus no intervention: Subgroup analysis by age at follow-up, Outcome 13 WAZ.

Analysis 4.14. Comparison 4 LNS versus no intervention: Subgroup analysis by age at follow-up, Outcome 14 WHZ.

Analysis 5.1. Comparison 5 LNS versus fortified blended food (FBF), Outcome 1 Moderate stunting.

Analysis 5.2. Comparison 5 LNS versus fortified blended food (FBF), Outcome 2 Severe stunting.

Analysis 5.3. Comparison 5 LNS versus fortified blended food (FBF), Outcome 3 Moderate wasting.

Analysis 5.4. Comparison 5 LNS versus fortified blended food (FBF), Outcome 4 Severe wasting.

Analysis 5.5. Comparison 5 LNS versus fortified blended food (FBF), Outcome 5 Moderate underweight.

Analysis 5.6. Comparison 5 LNS versus fortified blended food (FBF), Outcome 6 Severe underweight.

Analysis 5.7. Comparison 5 LNS versus fortified blended food (FBF), Outcome 7 MUAC.

Analysis 5.8. Comparison 5 LNS versus fortified blended food (FBF), Outcome 8 Haemoglobin (g/L).

Analysis 5.9. Comparison 5 LNS versus fortified blended food (FBF), Outcome $9 \mathrm{HAZ}$.

Analysis 5.10. Comparison 5 LNS versus fortified blended food (FBF), Outcome 10 WAZ.

Analysis 5.11. Comparison 5 LNS versus fortified blended food (FBF), Outcome 11 WHZ.

Analysis 6.1. Comparison 6 LNS versus micronutrient powders (MNP), Outcome 1 Moderate stunting.

Analysis 6.2. Comparison 6 LNS versus micronutrient powders (MNP), Outcome 2 Moderate wasting.

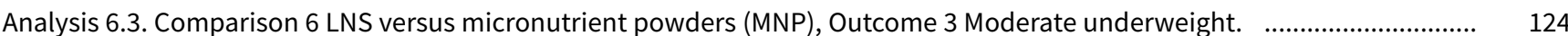

Analysis 6.4. Comparison 6 LNS versus micronutrient powders (MNP), Outcome 4 Anaemia.

Analysis 6.5. Comparison 6 LNS versus micronutrient powders (MNP), Outcome 5 Anaemia: Sensitivity analysis. ....................... 125

Analysis 6.6. Comparison 6 LNS versus micronutrient powders (MNP), Outcome 6 Serum haemoglobin (g/L). ........................... 125

Analysis 6.7. Comparison 6 LNS versus micronutrient powders (MNP), Outcome 7 Serum haemoglobin (g/L): Sensitivity 126 analysis.

Analysis 6.8. Comparison 6 LNS versus micronutrient powders (MNP), Outcome $8 \mathrm{HAZ}$.

Analysis 6.9. Comparison 6 LNS versus micronutrient powders (MNP), Outcome 9 HAZ: Sensitivity analysis. ..........................

Analysis 6.10. Comparison 6 LNS versus micronutrient powders (MNP), Outcome 10 WAZ.

Analysis 6.11. Comparison 6 LNS versus micronutrient powders (MNP), Outcome 11 WAZ: Sensitivity analysis.

Analysis 6.12. Comparison 6 LNS versus micronutrient powders (MNP), Outcome 12 WHZ.

Analysis 6.13. Comparison 6 LNS versus micronutrient powders (MNP), Outcome 13 WHZ: Sensitivity analysis. 
[Intervention Review]

\title{
Preventive lipid-based nutrient supplements given with complementary foods to infants and young children 6 to 23 months of age for health, nutrition, and developmental outcomes
}

\author{
Jai K Das ${ }^{1}$, Rehana A Salam¹, Yousaf Bashir Hadi², Sana Sadiq Sheikh¹, Afsah Z Bhutta ${ }^{3}$, Zita Weise Prinzo ${ }^{4}$, Zulfiqar A Bhutta5,6 \\ 1Division of Women and Child Health, Aga Khan University Hospital, Karachi, Pakistan. 2Department of Internal Medicine, West Virginia \\ University, Morgantown, West Virginia, USA. ${ }^{3}$ Dow University of Health Sciences, Karachi, Pakistan. ${ }^{4}$ Department of Nutrition for Health \\ and Development, World Health Organization, Geneva, Switzerland. ${ }^{5}$ Centre for Global Child Health, The Hospital for Sick Children, \\ Toronto, Canada. ${ }^{6}$ Center for Excellence in Women and Child Health, Aga Khan University Hospital, Karachi, Pakistan
}

Contact address: Zulfiqar A Bhutta, Center for Excellence in Women and Child Health, Aga Khan University Hospital, Karachi, Pakistan. Zulfiqar.bhutta@sickkids.ca,zulfiqar.bhutta@aku.edu.

Editorial group: Cochrane Developmental, Psychosocial and Learning Problems Group.

Publication status and date: Edited (no change to conclusions), published in Issue 5, 2019.

Citation: Das JK, Salam RA, Hadi YB, Sadiq Sheikh S, Bhutta AZ, Weise Prinzo Z, Bhutta ZA. Preventive lipid-based nutrient supplements given with complementary foods to infants and young children 6 to 23 months of age for health, nutrition, and developmental outcomes. Cochrane Database of Systematic Reviews 2019, Issue 5. Art. No.: CD012611. DOI: 10.1002/14651858.CD012611.pub3.

Copyright ( 2019 The Cochrane Collaboration. Published by John Wiley \& Sons, Ltd.

\section{A B S T R A C T}

\section{Background}

One nutritional intervention advocated to prevent malnutrition among children is lipid-based nutrient supplements (LNS). LNS provide a range of vitamins and minerals, but unlike most other micronutrient supplements, LNS also provide energy, protein and essential fatty acids. Alternative recipes and formulations to LNS include fortified blended foods (FBF), which are foods fortified with vitamins and minerals, and micronutrient powders (MNP), which are a combination of vitamins and minerals,

\section{Objectives}

To assess the effects and safety of preventive LNS given with complementary foods on health, nutrition and developmental outcomes of non-hospitalised infants and children six to 23 months of age, and whether or not they are more effective than other foods (including FBF or MNP).

This review did not assess the effects of LNS as supplementary foods or therapeutic foods in the management of moderate and severe acute malnutrition.

\section{Search methods}

In October 2018, we searched CENTRAL, MEDLINE, Embase, 21 other databases and two trials registers for relevant studies. We also checked the reference lists of included studies and relevant reviews and contacted the authors of studies and other experts in the area for any ongoing and unpublished studies.

\section{Selection criteria}

Randomised controlled trials (RCTs) and quasi-RCTs that evaluated the impact of LNS plus complementary foods given at point-of-use (for any dose, frequency, duration) to non-hospitalised infants and young children aged six to 23 months in stable or emergency settings and compared to no intervention, other supplementary foods (i.e. FBF), nutrition counselling or multiple micronutrient supplements or powders for point-of-use fortification of complementary foods. 


\section{Data collection and analysis}

Two review authors independently screened studies for relevance and, for those studies included in the review, extracted data, assessed risk of bias and rated the quality of the evidence using the GRADE approach. We carried out statistical analysis using Review Manager software. We used a random-effects meta-analysis for combining data as the interventions differed significantly. We set out the main findings of the review in 'Summary of findings' tables,.

\section{Main results}

Our search identified a total of 8124 records, from which we included 17 studies ( 54 papers) with 23,200 children in the review. The included studies reported on one or more of the pre-specified primary outcomes, and five studies included multiple comparison groups.

Overall, the majority of trials were at low risk of bias for random sequence generation, allocation concealment, blinding of outcome assessment, incomplete outcome data, selective reporting and other sources of bias, but at high risk of bias for blinding of participants and personnel due to the nature of the intervention. Using the GRADE approach, we judged the quality of the evidence for most outcomes as low or moderate.

LNS+complementary feeding compared with no intervention Thirteen studies compared LNS plus complementary feeding with no intervention. LNS plus complementary feeding reduced the prevalence of moderate stunting by $7 \%$ (risk ratio (RR) $0.93,95 \%$ confidence interval ( $\mathrm{Cl}) 0.88$ to 0.98 ; nine studies, 13,372 participants; moderate-quality evidence), severe stunting by $15 \%(\mathrm{RR} 0.85,95 \% \mathrm{Cl} 0.74$ to 0.98 ; five studies, 6151 participants; moderate-quality evidence), moderate wasting by $18 \%$ (RR $0.82,95 \% \mathrm{Cl} 0.74$ to 0.91 ; eight studies; 13,172 participants; moderate-quality evidence), moderate underweight by $15 \%$ (RR $0.85,95 \% \mathrm{Cl} 0.80$ to 0.91 ; eight studies, 13,073 participants; moderate-quality evidence), and anaemia by $21 \%$ (RR $0.79,95 \% \mathrm{Cl} 0.69$ to 0.90 ; five studies, 2332 participants; low-quality evidence). There was no impact of LNS plus complementary feeding on severe wasting (RR 1.27, $95 \% \mathrm{Cl} 0.66$ to 2.46; three studies, 2329 participants) and severe underweight (RR $0.78,95 \% \mathrm{Cl} 0.54$ to 1.13 ; two studies, 1729 participants). Adverse effects did not differ between the groups (RR $0.86,95 \% \mathrm{Cl} 0.74$ to 1.01 ; three studies, 3382 participants).

LNS+complementary feeding compared with FBF Five studies compared LNS plus complementary feeding with other FBF, including corn soy blend and UNIMIX. We pooled four of the five studies in meta-analyses and found that, when compared to other FBF, LNS plus complementary feeding significantly reduced the prevalence of moderate stunting ( $\mathrm{RR} 0.89,95 \% \mathrm{Cl} 0.82$ to 0.97 ; three studies, 2828 participants; moderate-quality evidence), moderate wasting (RR $0.79,95 \% \mathrm{Cl} 0.65$ to 0.97 ; two studies, 2290 participants; moderatequality evidence), and moderate underweight (RR $0.81,95 \% \mathrm{Cl} 0.73$ to 0.91 ; two studies, 2280 participants; moderate-quality evidence). We found no difference between LNS plus complementary feeding and FBF for severe stunting (RR $0.41,95 \% \mathrm{Cl} 0.12$ to 1.42 ; two studies, 729 participants; low-quality evidence), severe wasting (RR $0.64,95 \% \mathrm{Cl} 0.19$ to 2.81 ; two studies, 735 participants; moderate-quality evidence), and severe underweight (RR $1.23,95 \% \mathrm{Cl} 0.67$ to 2.25 ; one study, 173 participants; low-quality evidence).

LNS+complementary feeding compared with MNP Four studies compared LNS plus complementary feeding with MNP. We pooled data from three of the four studies in meta-analyses and found that compared to MNP, LNS plus complementary feeding significantly reduced the prevalence of moderate underweight ( $\mathrm{RR} 0.88,95 \% \mathrm{Cl} 0.78$ to 0.99; two studies, 2004 participants; moderate-quality evidence) and anaemia (RR $0.38,95 \% \mathrm{Cl} 0.21$ to 0.68 ; two studies, 557 participants; low-quality evidence). There was no difference between LNS plus complementary feeding and MNP for moderate stunting (RR $0.92,95 \% \mathrm{Cl} 0.82$ to 1.02; three studies, 2365 participants) and moderate wasting (RR $0.97,95 \% \mathrm{Cl} 0.77$ to 1.23 ; two studies, 2004 participants).

\section{Authors' conclusions}

The findings of this review suggest that LNS plus complementary feeding compared to no intervention is effective at improving growth outcomes and anaemia without adverse effects among children aged six to 23 months in low- and middle-income countries (LMIC) in Asia and Africa, and more effective if provided over a longer duration of time (over 12 months). Limited evidence also suggests that LNS plus complementary feeding is more effective than FBF and MNP at improving growth outcomes.

\section{PLAIN LANGUAGE SUMMARY}

\section{Effect of lipid-based nutrient supplementation on infants and young children}

\section{Review question}

What is the impact of lipid-based nutrient supplements (LNS) plus complementary foods on health, nutrition and developmental outcomes among infants and young children?

\section{Background}

LNS are food products which contain energy, minerals and vitamins that can improve growth in children. LNS provide vitamins, minerals and energy in the form of protein and essential fatty acids. We sought to assess the effect of LNS given jointly with complementary feeding, compared to no intervention, micronutrient powders (MNP; a mixture of vitamins and minerals that is sprinkled onto food) and other fortified blended food (FBF) products in healthy children. 


\section{Study characteristics}

This review includes 17 studies (from 54 reports) with 23,200 children. Four of the included studies were conducted in Malawi, three in Bangladesh, two in Ghana and one each in Burkina Faso, Haiti, Honduras, Chad, Congo, Kenya, Niger, Peru, Guatemala, and Indonesia. Four included studies enrolled pregnant women and provided LNS plus complementary feeding during pregnancy and post-partum, followed by infant supplementation starting at six months of age. The other studies provided LNS plus complementary feeding to children after six months of age. None of the included studies were conducted in emergency settings.

\section{Key results}

Findings of this review suggest that LNS plus complementary feeding is probably an effective intervention for improving growth outcomes and reducing the occurrence of children who are of short stature for their age (stunting), have low weight for their age (moderate underweight), have low weight for their height (moderate wasting) and anaemia. Additionally, LNS plus complementary feeding probably improves height and weight for age as well as mid-upper arm circumference without adverse effects among children aged six to 23 months. The intervention seems to be more effective if provided for a duration longer than 12 months.

Evidence also suggests that LNS plus complementary probably reduces moderate stunting, moderate wasting and moderate underweight, compared to other FBF.

Furthermore, LNS plus complementary feeding is probably more effective than MNP at reducing moderate underweight and improving height and weight.

\section{Quality of evidence}

Overall, we considered most studies to be at high risk of bias for blinding of participants and personnel due to the nature of intervention. We rated the quality of the evidence for most outcomes as either low or moderate.

\section{Currentness of evidence}

The evidence is current to October 2018. 
SUMMARY OF FINDINGS

Summary of findings for the main comparison.

LNS plus complementary feeding compared with no intervention

Patient or population: children aged 6 to 23 months

Settings: community

Intervention: LNS plus complementary feeding

Comparison: no intervention

\begin{tabular}{|c|c|c|c|c|c|c|}
\hline \multirow[t]{3}{*}{ Outcomes } & \multicolumn{2}{|c|}{$\begin{array}{l}\text { Illustrative comparative risks* } \\
(95 \% \mathrm{Cl})\end{array}$} & \multirow[t]{3}{*}{$\begin{array}{l}\text { Relative effect } \\
(95 \% \mathrm{CI})\end{array}$} & \multirow{3}{*}{$\begin{array}{l}\text { Number of par- } \\
\text { ticipants } \\
\text { (studies) }\end{array}$} & \multirow{3}{*}{$\begin{array}{l}\text { Quality of the } \\
\text { evidence } \\
\text { (GRADE) }\end{array}$} & \multirow[t]{3}{*}{ Comments } \\
\hline & Assumed risk & $\begin{array}{l}\text { Corresponding } \\
\text { risk }\end{array}$ & & & & \\
\hline & $\begin{array}{l}\text { No interven- } \\
\text { tion }\end{array}$ & $\begin{array}{l}\text { LNS plus com- } \\
\text { plementary } \\
\text { feeding }\end{array}$ & & & & \\
\hline \multicolumn{7}{|l|}{ Stunting } \\
\hline $\begin{array}{l}\text { Moderate stunting } \\
\text { Measured as height-for-age } z \text { score }<-2 \\
\text { SD }\end{array}$ & $2618 / 7137$ & $2353 / 7060$ & $\begin{array}{l}\mathbf{R R} 0.93 \text { (0.88 to } \\
0.98 \text { ) }\end{array}$ & $\begin{array}{l}13,372 \\
\text { (9 studies) }\end{array}$ & $\begin{array}{l}\oplus \oplus \oplus \ominus \\
\text { Moderate }^{a}\end{array}$ & $\begin{array}{l}2 \text { studies, Kumwenda } 2014 \text { and } \\
\text { Mangani } 2015 \text {, contributed data } \\
\text { to multiple comparisons; total } \\
\text { number of comparisons = } 13\end{array}$ \\
\hline \multicolumn{7}{|l|}{ Measured at 12,18 and 24 months of age } \\
\hline \multicolumn{7}{|l|}{ Measured at 12,18 and 24 months of age } \\
\hline \multicolumn{7}{|l|}{ Wasting } \\
\hline $\begin{array}{l}\text { Moderate wasting } \\
\text { Measured as weight-for-height z score < } \\
-2 \text { SD }\end{array}$ & $695 / 6213$ & $624 / 6959$ & $\begin{array}{l}\mathbf{R R} 0.82(0.74 \text { to } \\
0.91)\end{array}$ & $\begin{array}{l}13,172 \\
\text { (8 studies) }\end{array}$ & $\begin{array}{l}\oplus \oplus \oplus \ominus \\
\text { Moderate } a\end{array}$ & $\begin{array}{l}1 \text { study, Kumwenda 2014, con- } \\
\text { tributed data to } 2 \text { comparisons; } \\
\text { total number of comparisons = } \\
11\end{array}$ \\
\hline
\end{tabular}




\begin{tabular}{|c|c|c|c|c|c|c|c|}
\hline & Measured at 12,18 and 24 months of age & & & & & & \\
\hline 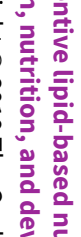 & $\begin{array}{l}\text { Severe wasting } \\
\text { Measured as weight-for-height } z \text { score }< \\
-3 \text { SD } \\
\text { Measured at } 12 \text { and } 18 \text { months of age }\end{array}$ & $18 / 1636$ & $21 / 1663$ & $\begin{array}{l}\text { RR } 1.27 \text { ( } 0.66 \text { to } \\
2.46)\end{array}$ & $\begin{array}{l}2329 \\
\text { (3 studies) }\end{array}$ & $\begin{array}{l}\oplus \oplus \oplus \oplus \\
\text { Moderateb }\end{array}$ & $\begin{array}{l}1 \text { study, Kumwenda 2014, con- } \\
\text { tributed data to } 4 \text { comparisons; } \\
\text { total number of comparisons = } \\
6\end{array}$ \\
\hline & Underweight & & & & & & \\
\hline 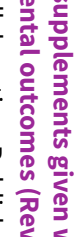 & $\begin{array}{l}\text { Moderate underweight } \\
\text { Measured as weight-for-age } z \text { score }<-2 \\
\text { SD } \\
\text { Measured at } 12,18 \text { and } 24 \text { months of age }\end{array}$ & $1723 / 7013$ & $1525 / 6861$ & $\begin{array}{l}\mathbf{R R} \mathbf{0 . 8 5} \text { (0.80 to } \\
0.91)\end{array}$ & $\begin{array}{l}13,073 \\
\text { (8 studies) }\end{array}$ & $\begin{array}{l}\oplus \oplus \oplus \ominus \\
\text { Moderate }^{a}\end{array}$ & $\begin{array}{l}1 \text { study, Kumwenda 2014, con- } \\
\text { tributed data to } 4 \text { comparisons; } \\
\text { total number of comparisons = } \\
11\end{array}$ \\
\hline 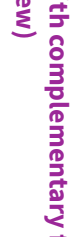 & $\begin{array}{l}\text { Severe underweight } \\
\text { Measured as weight-for-age } z \text { score }<-3 \\
\text { SD } \\
\text { Measured at } 12 \text { and } 18 \text { months of age }\end{array}$ & $62 / 1224$ & $50 / 1258$ & $\begin{array}{l}\text { RR } 0.78 \text { (0.54 to } \\
1.13)\end{array}$ & $\begin{array}{l}1729 \\
\text { (2 studies) }\end{array}$ & $\begin{array}{l}\oplus \oplus \oplus \oplus \\
\text { Moderate }\end{array}$ & $\begin{array}{l}1 \text { study, Kumwenda 2014, con- } \\
\text { tributed data to } 4 \text { comparisons; } \\
\text { total number of comparisons = } \\
5\end{array}$ \\
\hline 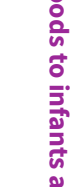 & $\begin{array}{l}\text { Anaemia } \\
\text { Measured as haemoglobin }<10 \mathrm{~g} / \mathrm{dL} \\
\text { Measured at } 12,18 \text { and } 24 \text { months of age }\end{array}$ & $697 / 1359$ & $558 / 973$ & $\begin{array}{l}\text { RR } 0.79 \text { (0.69 to } \\
0.90)\end{array}$ & $\begin{array}{l}2332 \\
\text { (5 studies) }\end{array}$ & $\begin{array}{l}\oplus \oplus \ominus \ominus \\
\text { Low }^{\mathbf{a}, \mathbf{c}}\end{array}$ & - \\
\hline 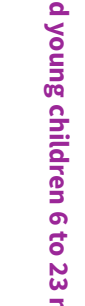 & $\begin{array}{l}\text { Adverse effects } \\
\text { Defined as deaths, hospitalisations, con- } \\
\text { genital abnormalities and life-threaten- } \\
\text { ing conditions requiring an immediate } \\
\text { hospital visit } \\
\text { Assessed at } 12 \text { and } 18 \text { months of age }\end{array}$ & $314 / 1369$ & $401 / 2333$ & $\begin{array}{l}\mathbf{R R} 0.86 \text { (0.74 to } \\
1.01)\end{array}$ & $\begin{array}{l}3382 \\
\text { (3 studies) }\end{array}$ & $\begin{array}{l}\oplus \oplus \oplus \ominus \\
\text { Moderate } a\end{array}$ & $\begin{array}{l}1 \text { study, Kumwenda 2014, con- } \\
\text { tributed data to } 2 \text { comparisons; } \\
\text { total number of comparisons = } \\
4\end{array}$ \\
\hline 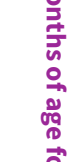 & $\begin{array}{l}\text { *The basis for the assumed risk (e.g. the } n \\
\text { sumed risk in the comparison group and t } \\
\text { Cl: confidence interval; LNS: lipid-based n }\end{array}$ & $\begin{array}{l}\text { hedian cont } \\
\text { he relative } \\
\text { utrient sup }\end{array}$ & $\begin{array}{l}p \text { risk across } \\
f \text { the interven } \\
\text {; RR: risk rati }\end{array}$ & $\begin{array}{l}\text { is provided in fo } \\
\text { hd its } 95 \% \mathrm{Cl}) \text {. } \\
\text { tandard deviatio }\end{array}$ & notes. The & onding risk & ts $95 \% \mathrm{Cl}$ ) is based on the as- \\
\hline
\end{tabular}

GRADE Working Group grades of evidence

High quality: Further research is very unlikely to change our confidence in the estimate of effect.

Moderate quality: Further research is likely to have an important impact on our confidence in the estimate of effect and may change the estimate. 


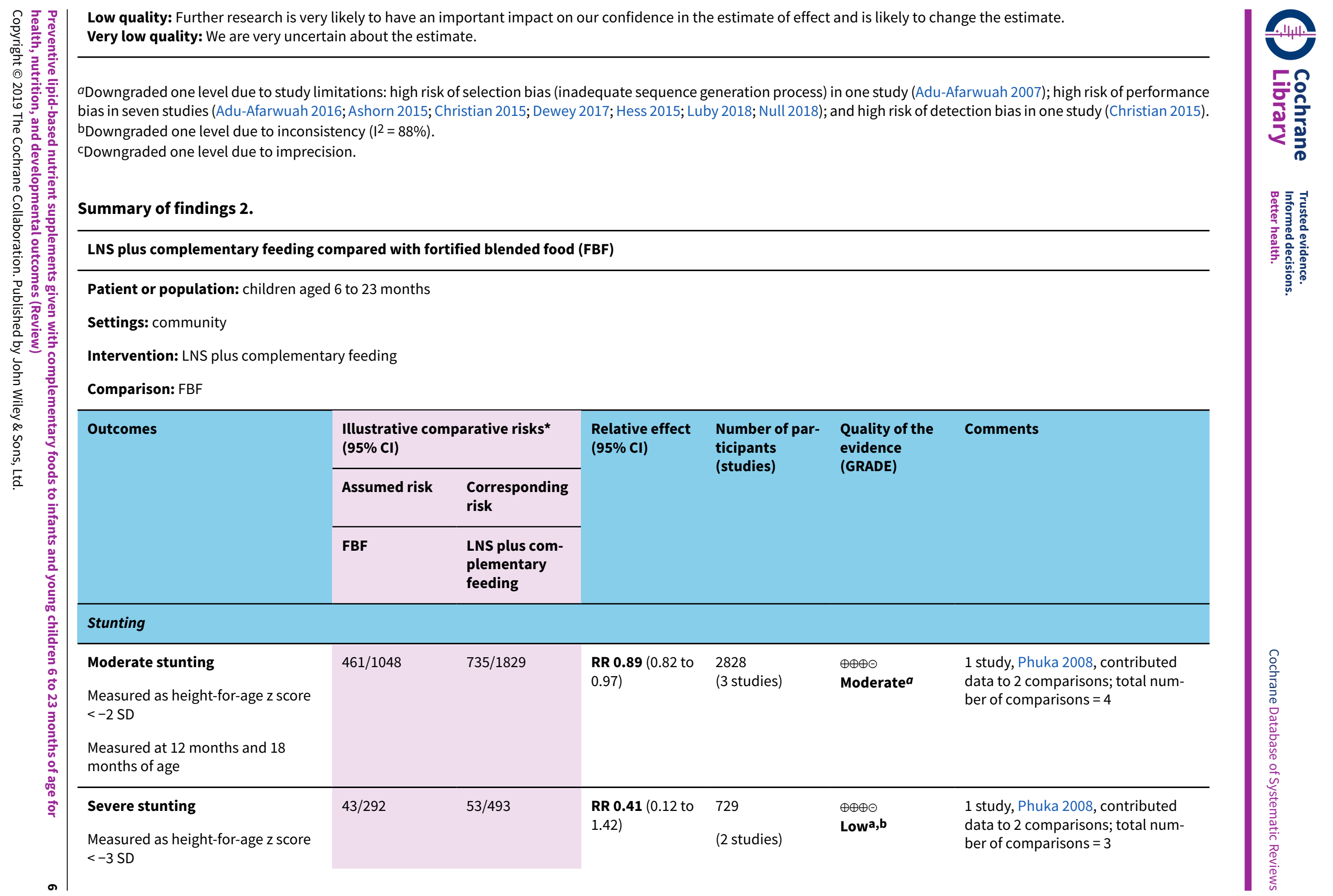




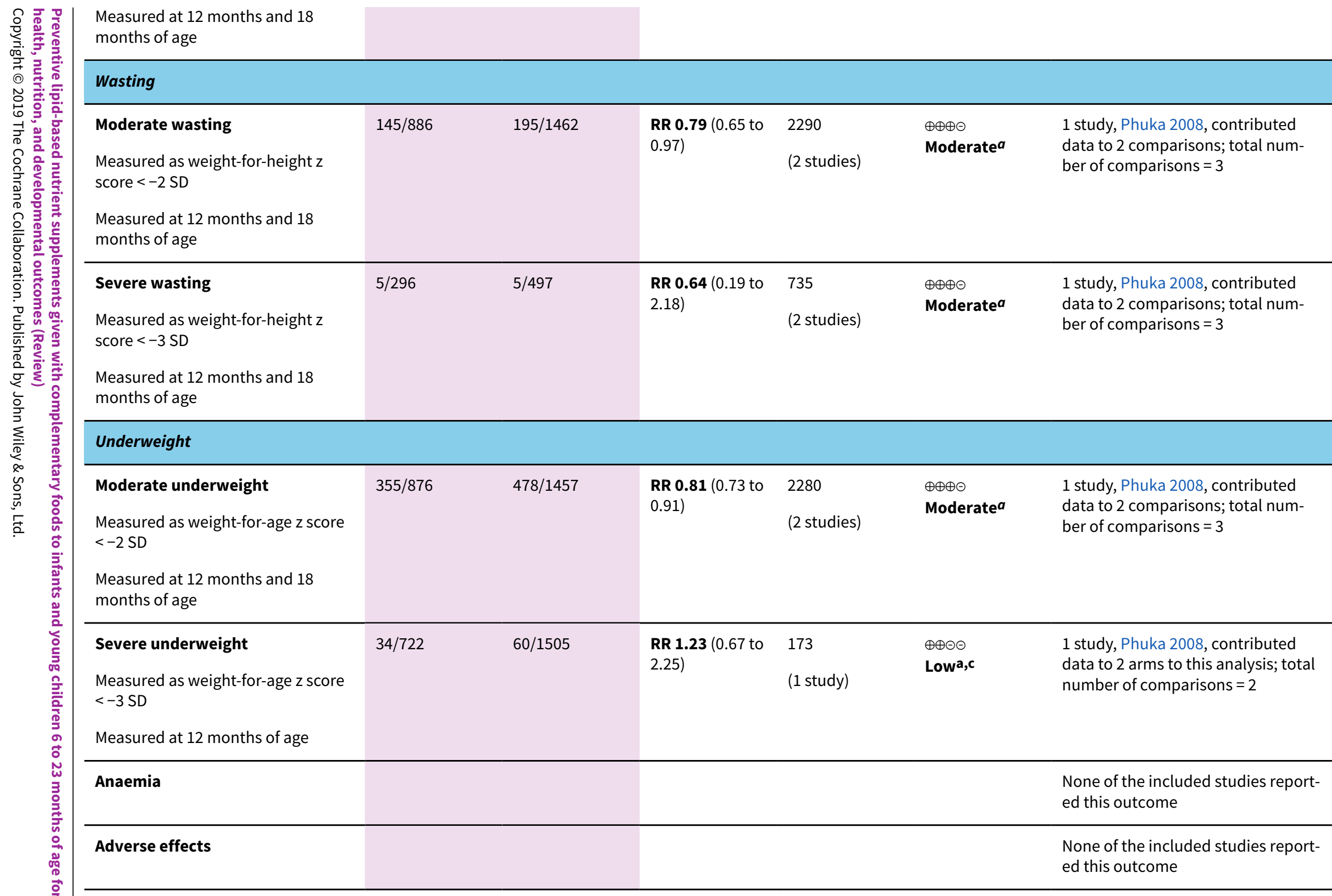

*The basis for the assumed risk (e.g. the median control group risk across studies) is provided in footnotes. The corresponding risk (and its $95 \% \mathrm{Cl}$ ) is based on the assumed risk in the comparison group and the relative effect of the intervention (and its $95 \% \mathrm{Cl}$ ). 
GRADE Working Group grades of evidence

High quality: further research is very unlikely to change our confidence in the estimate of effect

Moderate quality: further research is likely to have an important impact on our confidence in the estimate of effect and may change the estimate

Low quality: further research is very likely to have an important impact on our confidence in the estimate of effect and is likely to change the estimate

Very low quality: we are very uncertain about the estimate

aDowngraded one level due to study limitations: high risk of performance bias in two studies (Christian 2015; Phuka 2008).

bDowngraded one level due to imprecision (high heterogeneity; $\left.\right|^{2}=57 \%$ ).

cDowngraded one level due to small sample size.

\section{Summary of findings 3.}

\section{LNS plus complementary feeding compared with micronutrient powders (MNP)}

Patient or population: children aged 6 to 23 months

Settings: community

Intervention: LNS plus complementary feeding

Comparison: MNP

\begin{tabular}{|c|c|c|c|c|c|c|}
\hline \multirow[t]{3}{*}{ Outcomes } & \multicolumn{2}{|c|}{$\begin{array}{l}\text { Illustrative comparative risks* } \\
(95 \% \mathrm{Cl})\end{array}$} & \multirow[t]{3}{*}{$\begin{array}{l}\text { Relative effect } \\
(95 \% \mathrm{CI})\end{array}$} & \multirow{3}{*}{$\begin{array}{l}\text { Number of par- } \\
\text { ticipants } \\
\text { (studies) }\end{array}$} & \multirow{3}{*}{$\begin{array}{l}\text { Quality of the } \\
\text { evidence } \\
\text { (GRADE) }\end{array}$} & \multirow[t]{3}{*}{ Comments } \\
\hline & Assumed risk & $\begin{array}{l}\text { Corresponding } \\
\text { risk }\end{array}$ & & & & \\
\hline & MNP & $\begin{array}{l}\text { LNS plus com- } \\
\text { plementary } \\
\text { feeding }\end{array}$ & & & & \\
\hline $\begin{array}{l}\text { Moderate stunting } \\
\text { Measured as height-for-age z score }<-2 \text { SD }\end{array}$ & $421 / 1059$ & $336 / 945$ & $\begin{array}{l}\text { RR } 0.92 \text { ( } 0.82 \text { to } \\
1.02)\end{array}$ & $\begin{array}{l}2365 \\
\text { (3 studies) }\end{array}$ & $\begin{array}{l}\oplus \oplus \oplus \ominus \\
\text { Moderate } a\end{array}$ & - \\
\hline Measured at 12 months and 24 months of age & & & & & & \\
\hline $\begin{array}{l}\text { Moderate wasting } \\
\text { Measured as weight-for-height } z \text { score }<-2 \text { SD }\end{array}$ & $133 / 1059$ & $115 / 945$ & $\begin{array}{l}\text { RR } 0.97 \text { (0.77 to } \\
1.23 \text { ) }\end{array}$ & $\begin{array}{l}2004 \\
\text { (2 studies) }\end{array}$ & $\begin{array}{l}\oplus \oplus \oplus \ominus \\
\text { Moderate }^{a}\end{array}$ & - \\
\hline
\end{tabular}

Measured as weight-for-height z score $<-2$ SD 
Measured at 12 months and 24 months of age

\section{Moderate underweight}

$376 / 1059$

$292 / 945$

RR 0.88 (0.78 to

2004

( 2 studies)

$\oplus \oplus \oplus \odot$

Moderate $a$

Measured as weight-for-age $z$ score $<-2$ SD

Measured at 12 months and 24 months of age

\section{Anaemia}

$18 / 98$

$10 / 98$

RR 0.38 ( 0.21 to

557

Measured as haemoglobin $<10 \mathrm{~g} / \mathrm{dL}$

0.68 )

(2 studies)

$\oplus \oplus \ominus \ominus$

Lowa,b

Measured at 12 months of age

\section{Adverse effects}

None of the in-

cluded studies

reported this

outcome

*The basis for the assumed risk (e.g. the median control group risk across studies) is provided in footnotes. The corresponding risk (and its $95 \% \mathrm{Cl}$ ) is based on the assumed risk in the comparison group and the relative effect of the intervention (and its $95 \% \mathrm{Cl}$ ).

CI: Confidence interval; LNS: Lipid-based nutrient supplements; RR: Risk ratio; SD: Standard deviation.

GRADE Working Group grades of evidence

High quality: further research is very unlikely to change our confidence in the estimate of effect

Moderate quality: further research is likely to have an important impact on our confidence in the estimate of effect and may change the estimate

Low quality: further research is very likely to have an important impact on our confidence in the estimate of effect and is likely to change the estimate

Very low quality: we are very uncertain about the estimate

aDowngraded one level due to study limitations: high risk of performance bias in two studies (Adu-Afarwuah 2016; Dewey 2017).

bDowngraded one level due to small sample size. 


\section{B A C K G R O U N D}

\section{Description of the condition}

Each year, malnutrition - including fetal growth restriction, stunting, wasting and micronutrient deficiencies -and suboptimum breastfeeding underlie nearly 3.1 million deaths of children under the age of five years worldwide, accounting for $45 \%$ of all deaths in this age group (Liu 2012). Globally in 2011, at least 165 million children were stunted (below -2 standard deviations (SD) from median height for age of reference population) and 52 million were wasted (below - 2 SD from median weight for height of reference population). Although the prevalence of stunting has decreased during the past two decades, it remains higher in South Asia (27\%) and Sub-Saharan Africa (36\%) compared to high-income countries (7\%) (Black 2013; WHO 2014). Micronutrient deficiencies are also prevalent in children and these deficiencies are associated with learning disability, impaired work capacity and increased morbidity and mortality in adulthood (Black 2013). Undernutrition among children has also been associated with delayed or compromised motor and cognitive development, decreased school achievement, and consequently, reduced economic productivity (The World Bank 2006).

Disruption and displacement of populations in emergency situations pose an added threat to the existing situation of malnutrition among children. Women and children represented over three-quarters of the estimated 80 million people in need of humanitarian assistance in 2014, and many countries with high maternal, newborn and child mortality rates are affected by humanitarian emergencies (UNICEF 2014). Malnutrition has been recorded as either a direct or an underlying cause of child mortality in emergencies (UNICEF 2014).

In order for countries to meet global targets for improved maternal, infant and child nutrition, there is a need to emphasise early prevention to address general deprivation and inequity for sustainable reductions in malnutrition (WHO 2014). Prevention is emphasised because the extent to which the effects of early damage are reversible is a complex area of ongoing research (Victora 2008). The concept of a preventive role of nutrition interventions is complex in settings with existing widespread and chronic undernutrition. However, the preventive approach aims to improve the daily nutritional intake by providing required calories, minerals and vitamins, and hence preventing undernutrition among children in vulnerable settings.

\section{Description of the intervention}

Ideally, infants are breastfed for two years or longer, with complementary food introduced at six months of age (WHO 2014). Diets of infants and young children aged six to 23 months need to include a variety of nutrient-dense foods, preferably from local sources, to ensure their nutrient needs are met (WHO 2014). However, children's diets are likely to be deficient in macronutrients and micronutrients, specifically essential fatty acids, when nutrient-rich diets are not available to them in resource-poor settings (Arimond 2015). Various interventions are recommended, or have been used, to improve child malnutrition, including improved maternal nutrition, promotion of breast feeding, appropriate complementary feeding and prophylactic vitamin $\mathrm{A}$ and zinc supplementation in children, along with other indirect interventions, including agricultural and financial interventions (Bhutta 2013).

Supplementary feeding is a strategy that includes provision of extra food to children beyond the normal ration of their home diets and is aimed at improving the nutritional status or preventing the nutritional deterioration of the target population. One of the nutritional interventions advocated to address malnutrition among children is lipid-based nutrient supplements (LNS). LNS are a family of products designed to deliver nutrients to vulnerable people. They are considered 'lipid-based' because most of the energy provided by these products is from lipids (fats). All LNS provide a range of vitamins and minerals, but unlike most other micronutrient supplements, LNS also provide energy, protein and essential fatty acids (Chaparro 2010; Ilins 2015). LNS recipes can include a variety of ingredients, but typically have included vegetable fat, peanut or groundnut paste, milk powder and sugar. Based on the energy content, LNS can be small quantity (SQ LNS) providing 110 to $120 \mathrm{kcal} /$ day (20 g dose), medium quantity (MQ LNS) providing 250 to $500 \mathrm{kcal} /$ day (45 g to $90 \mathrm{~g}$ dose) or largequantity (LQ LNS) providing more than $280 \mathrm{kcal} /$ day (> $90 \mathrm{~g}$ dose) (WHO 2012; WHO 2013). LNS are nutrient dense, require no cooking before use, and can be stored for months even in warm conditions (Phuka 2008).

Alternative recipes and formulations, other than LNS, are currently being explored using cereals mixed with other ingredients, including whey, soy protein isolate, dried skimmed milk, and sesame, cashew and chickpea paste, among others (Pee 2008). These are fortified with vitamins and minerals and are commonly called fortified blended foods (FBF). An example of a commonly used FBF is corn soy blend plus (CSB ++), which is a cooked blend of milled, heat-treated corn and soybeans that is fortified with a vitamin and mineral premix. Multiple micronutrient powders (MNP) are also an alternative way of providing micronutrients. These are single-dose packets of vitamins and minerals in powder form that can be sprinkled onto any ready to eat semi-solid food consumed at home, school or any other point of use. The World Health Organization (WHO) recommends home fortification of foods with multiple MNP to improve iron status and reduce anaemia among infants and children aged six to 23 months of age where the prevalence of anaemia in children under five years of age is $20 \%$ or higher (WHO 2011). Besides complementary and supplementary feeding interventions, nutrition education and counselling provided to caregivers on the feeding of young children also have the potential to improve the nutritional status of children in developing countries (Lassi 2013). Nutrition education alone for improving complementary feeding practices in both food-secure and food-insecure populations has been shown to improve feeding practices and improve growth and anthropometric measures (Lassi 2013).

Though LNS or any other fortified food is not a replacement for breast milk or a diverse diet of local foods, the use of LNS for pointof-use fortification of complementary foods in infants aged six to 23 months has been proposed as a promising intervention for the prevention of malnutrition in vulnerable settings. LNS products are specifically designed to ensure nutrient adequacy (energy, protein and essential fatty acids), while simultaneously upholding other complementary feeding practices such as breastfeeding and dietary diversity. 


\section{How the intervention might work}

The scope of this review is limited to assessing the effects of LNS for the prevention of malnutrition when given to children aged six to 23 months in addition to complementary foods. We did not assess the role of LNS as supplementary foods or therapeutic foods for the management of moderate and severe acute malnutrition.

LNS work by supplementing children with the required nutrients, mainly from lipids, and include energy, protein, essential fatty acids, and micronutrients in addition to the normal home diet. They provide calories as well as micronutrients. The doses and formulations of LNS can be modified according to the needs of the specific target group and, to date, there is no standard formulation (Dewey 2012). The supplements can be modified by adjusting the macronutrient content to maximise palatability and texture, and adding flavours according to regional taste preferences. It is further suggested that LNS are provided in single-serving sachets to encourage thinking of it as a condiment, a medicine or a special food for a special group. Serving LNS in a single serving also prevents interfamily sharing since programmatic findings from studies suggest that there are issues with redistribution of the supplement within the family when feeding is homedelivered, and much less leakage when delivered in day care centres (Kristjansson 2015). During manufacture, international guidelines need to be followed to prevent faecal contamination and fat oxidation to enhance shelf life (WHO 2012). The most commonly used formulations of fortified complementary food supplementation are Nutributter ${ }^{\circledR}(20 \mathrm{~g}$ or $108 \mathrm{kcal}$ per day) and Plumpy'doz ${ }^{\circledR}$ (46 g or $246 \mathrm{kcal}$ per day), both of which are produced by Nutriset, Malaunay, France, and 'fortified spreads' $(25 \mathrm{~g}$ to $75 \mathrm{~g}$ or 128 to $384 \mathrm{kcal}$ per day).

Studies have also explored the acceptability of LNS among infants (Adu-Afarwuah 2011; Arimond 2015; Hess 2011), and suggest that mothers found it convenient to use, as it could be mixed with any food they preferred, and that the use of LNS could be made simpler by packaging the supplement in convenient daily doses. This shows that acceptability of LNS is similar to that of MNP, but that LNS can potentially also address general calorie deficit.

\section{Why it is important to do this review}

Recent research on smaller doses of LNS for the prevention of malnutrition has created interest in their potential use to ensure a nutritionally adequate ration for the most vulnerable groups, including children between six and 23 months of age (Chaparro 2010; Dewey 2012). Studies have shown mixed results for the impact of LNS on growth and development in infants and young children (Huybregts 2012; lannotti 2013; Maleta 2015; Mangani 2013; Mangani 2015; Prado 2016; Thakwalakwa 2012; Thakwalakwa 2015). Furthermore, there could be potential concerns relating to LNS safety in areas where infections are common (De-Regil 2013). A study from Malawi suggested that LNS containing iron did not increase morbidity in children and also did not affect guardian-reported illness episodes, but may have increased malaria-related non-scheduled visits in one of the intervention groups (Bendabenda 2016). Other studies have suggested perceived benefits of LNS by care providers, including acceptability, adherence and willingness to pay (Iuel-Brockdorf 2015; Segrè 2015). Several countries are implementing largescale projects that involve the provision of LNS as part of the interventions. An implementation survey of these interventions identified 20 projects providing LNS interventions, mostly in Sub-Saharan Africa (UNICEF 2013). Of these, 17 were currently distributing LNS in 13 countries and three were planning to start distribution within the next 12 months. More than half (around $53 \%$ ) of the implemented projects providing LNS aimed to improve complementary feeding or to prevent and treat moderate acute malnutrition (MAM), while $41 \%$ had an objective to prevent and control micronutrient malnutrition and 35\% aimed to reduce stunting. Most of the planned LNS interventions that aimed to improve complementary feeding were integrated with existing infant and young child feeding programs, micronutrient prevention and control programs, humanitarian response programs and programs designed to prevent MAM (UNICEF 2013).

Reviews have evaluated supplementary feeding for children in the form of added meals, drinks or snacks, and suggest some benefit on child growth and psychosocial outcomes (Kristjansson 2015; Kristjansson 2016; Sguassero 2012). These reviews have assessed the effectiveness of community-based supplementary feeding interventions among children under five years of age in disadvantaged population groups. Findings from one review on supplementary feeding suggested that the key for successful feeding programs for young children in low- and middle-income countries (LMIC) is good implementation (Kristjansson 2015). To date, the benefits and harms of preventive LNS in infants and young children aged six to 23 months have not been systematically assessed. LNS products are more expensive to produce, transport and store compared to routine complementary food because of their composition, weight, and size. Hence, research is needed to determine the added benefit of LNS products for improved health and functional outcomes (UNICEF 2013), and studies are also needed to compare its effectiveness against natural local food sources. The retrieval, summary and assessment of the evidence for LNS compared to other supplements will assist international organisations and countries to make informed decisions about the benefits and harms of LNS in infants and young children when given with complementary foods. We have also developed a companion review on the effectiveness and safety of LNS when given to women during pregnancy on maternal, birth and infant outcomes (Das 2018), which will also guide policy makers in making informed decisions about the effectiveness and safety of LNS in pregnant women.

\section{O B JECTIVES}

To assess the effects and safety of preventive lipid-based nutrient supplements (LNS) given with complementary foods on health, nutrition and developmental outcomes of non-hospitalised infants and children six to 23 months of age, and whether or not LNS are more effective than other foods (including fortified blended foods (FBF) or multiple micronutrient powders (MNP)).

This review did not assess the effects of LNS as supplementary foods or therapeutic foods in the management of moderate and severe acute malnutrition.

\section{METHODS}

\section{Criteria for considering studies for this review}

\section{Types of studies}

Randomised controlled trials (RCTs) and quasi-RCTs. 


\section{Types of participants}

All non-hospitalised infants and young children aged six to 23 months of age in stable (i.e. not in any emergency-affected country or emergency settings according to WHO definition (Wisner 2002). We did not include infants under six months of age, as exclusive breastfeeding is recommended from birth to six months. We included studies of apparently healthy children* from the general population, although some might be at risk of having highly prevalent diseases such as malaria, diarrhoea or even malnutrition. We did not exclude studies with infants and children with HIV infection, unless they were hospitalised or had a clinical condition.

*Apparently health children are children who were described by the study authors as being healthy. We did not include studies specifically undertaken with diseased or undernourished populations.

\section{Types of interventions}

All infants and young children who were not wasted and who were given LNS with complementary food at point-of-use for any dose, frequency and duration compared to no intervention, placebo, or compared with other foods/supplements or nutrition intervention. Specifically, we made the following comparisons.

1. Provision of LNS versus no intervention or placebo

2. Provision of LNS versus other supplementary foods (i.e. FBF)

3. Provision of LNS versus nutritional counselling (counselling to mothers and caregivers for appropriate feeding of infants and young children)

4. Provision of LNS versus provision of multiple micronutrient supplements or powders for point-of-use fortification of complementary foods

We included interventions that combined provision of LNS with co-interventions, such as education or other approaches, if the other co-interventions were the same in both the intervention and comparison groups.

\section{Types of outcome measures}

\section{Primary outcomes}

1. Stunting (moderate: height/length-for-age $(\mathrm{HFA})<-2$ standard deviations (SD); severe: HFA <-3 SD)

2. Wasting (moderate: weight-for-height/length $(W F H)<-2$ SD; severe: $\mathrm{WFH}<-3 \mathrm{SD}$ )

3. Underweight (moderate: weight-for-age $(W F A)<-2$ SD; severe: WFA $<-3$ SD)

4. Anaemia (as defined by trialists)

5. Psychomotor development outcomes (as defined by trialists)

6. Neuro-developmental outcomes (as defined by trialists)

7. Any adverse effects, including allergic reactions, as diagnosed by clinical assessment (atopic dermatitis, urticaria, oedema (oral), ophthalmic pruritus, allergic rhinitis, asthma, anaphylaxis)

\section{Secondary outcomes}

1. Mid-upper arm circumference (MUAC; the circumference of the left upper arm, measured at the mid-point between the tip of the shoulder and the tip of the elbow)

2. Haemoglobin (g/L)
3. Morbidity (incidence of diarrhoea, acute respiratory illness (ARI) and fever, as defined by trialists)

4. Mortality

Explanatory secondary outcomes

1. Height/length-for-age $z$ score (HAZ)

2. Weight-for-age z score (WAZ)

3. Weight-for-height/length z score (WHZ)

\section{Search methods for identification of studies}

\section{Electronic searches}

We searched the sources listed below for intervention studies in March 2017, June 2018 and October 2018. On 5 March 2019, shortly before publication, we searched MEDLINE, Embase and the Retraction Watch Database retractiondatabase.org/ RetractionSearch.aspx? to identify any retraction statements or errata relating to the included studies. These searches are reported in Appendix 1

\section{International databases}

1. Cochrane Central Register of Controlled Trials (CENTRAL; 2017, Issue 2) in the Cochrane Library, and which includes the Cochrane Developmental, Psychosocial and Learning Problems Specialised Register (searched 16 October 2018).

2. MEDLINE Ovid (1946 to 15 October 2018).

3. MEDLINE In-Process and Other Non-Indexed Citations Ovid (searched 15 October 2018).

4. MEDLINE E-pub ahead of print Ovid (searched 15 October 2018).

5. Embase Ovid (1974 to 2018 week 42).

6. CINAHL EBSCOhost (Cumulative Index to Nursing and Allied Health Literature; 1937 to 16 October 2018).

7. Science Citation Index Web of Science (SCl; 1970 to 16 October 2018).

8. Social Sciences Citation Index Web of Science (SSCI; 1970 to 16 October 2018).

9. Conference Proceedings Citation Index - Science Web of Science (CPCl-S; 1990 to 16 October 2018).

10.Conference Proceedings Citation Index - Social Science \& Humanities Web of Science (CPCI-SS\&H; 1990 to 16 October 2018).

11.Cochrane Database of Systematic Reviews (CDSR; 2018, Issue 10), part of the Cochrane Library.

12.Database of Abstracts of Reviews of Effect (DARE; 2015, Issue 2) in the Cochrane Library. DARE ceased publication in 2015 (searched on 23 March 2017).

13.Epistemonikos (epistemonikos.org; searched 20 October 2018). 14.POPLINE (www.popline.org; searched 20 October 2018).

15.ClinicalTrials.gov (clinicaltrials.gov; searched 20 October 2018)

16. World Health Organization International Clinical Trials Registry Platform (WHO ICTRP; who.int/trialsearch; searched 20 October 2018).

\section{Regional databases}

1. IBECS (Índice Bibliográfico Español en Ciencias de la Salud;ibecs.isciii.es/cgi-bin/wxislind.exe/iah/online/? IsisScript=iah/iah.xis\&base=IBECS\&lang=p\&form=F; searched 19 October 2018).

Preventive lipid-based nutrient supplements given with complementary foods to infants and young children 6 to 23 months of age for 
2. SCiELO (Scientific Electronic Library Online; www.scielo.br; searched 19 October 2018).

3. AIM (Africa Global Index Medicus; search.bvsalud.org/ ghl/?lang=en\&submit=Search\&where=REGIONAL; searched 19 October 2018).

4. IMEMR (Index Medicus for the Eastern Mediterranean Region Global Index Medicus ; search.bvsalud.org/ ghl/?lang=en\&submit=Search\&where=REGIONAL; searched 19 October 2018).

5. LILACS (Latin American and Caribbean Health Science Information database; lilacs.bvsalud.org/en; searched 19 October 2018).

6. PAHO/WHO Institutional Repository for Information Sharing (iris.paho.org/xmlui; searched 19 October 2018).

7. WHOLIS Global Index Medicus (WHO Library Database; search.bvsalud.org/ghl/?

lang=en\&submit=Search\&where=REGIONAL; searched 19 October 2018).

8. WPRIM Global Index Medicus(Western Pacific Index Medicus; search.bvsalud.org/ghl/?

lang=en\&submit=Search\&where=REGIONAL; searched 19 October 2018).

9. IMSEAR Global Index Medicus (Index Medicus for the South-East Asian Region; search.bvsalud.org/ghl/? lang=en\&submit=Search\&where=REGIONAL; searched 19 October 2018).

10.IndMED (indmed.nic.in/indmed.html; searched 19 October 2018).

11.Native Health Research Database (hscssl.unm.edu/nhd; searched 19 October 2018).

We searched using both keywords and controlled vocabulary (when available), using the search strategies in Appendix 2. We did not apply language or date restrictions for any source. If we identified studies written in a language other than English, we commissioned their translation into English. If necessary, we would have recorded any such studies as 'Studies awaiting classification' until a translation became available.

\section{Searching other resources}

We checked the reference lists of included studies and relevant reviews for further studies. We contacted authors of eligible studies and other relevant persons for information about ongoing or unpublished studies we might have missed or, where necessary, to provide missing data (Dewey 2016 [pers comm]; Dewey 2017 [pers comm]; Stewart 2017 [pers comm]).

\section{Data collection and analysis}

\section{Selection of studies}

Two review authors independently assessed for inclusion all records generated by the search strategy. First, they screened titles and abstracts of all records retrieved, and short-listed those deemed relevant. Next, they obtained and assessed the full texts of all potentially relevant records, assessing each one against the inclusion criteria (Criteria for considering studies for this review), before deciding on the final list of studies to be included in the review. Both review authors resolved any disagreements regarding eligibility at each stage of the selection process through discussion or, if required, in consultation with a third author. We recorded our decisions in a PRISMA diagram (Moher 2009).

\section{Data extraction and management}

We designed a data extraction form specifically for this review (Appendix 3). Two review authors used the form to extract data on study methods, participants, intervention, control, reported outcomes, source of funding and potential conflict of interest statements from all included studies. If studies reported outcomes at multiple time points, we extracted data for each time point and pooled studies reporting similar outcomes at similar time points. When information was unclear, we attempted to contact the authors of the original report to request they provide further details. We used these details, which are presented in the Characteristics of included studies tables, to explore and make inferences for the results.

Two review authors entered the data into Review Manager 5 (RevMan 5) (Review Manager 2014), and a third review author checked the data entry for accuracy by entering the data into a separate file and comparing the results.

We resolved discrepancies at all stages through discussion or, if required, through consultation with a third review author.

\section{Assessment of risk of bias in included studies}

\section{Randomised studies}

Two review authors independently assessed the risk of bias of each included study using the criteria outlined in the Cochrane Handbook for Systematic Reviews of Interventions (Higgins 2011a) and set out in Appendix 4. We rated each study at high, low or unclear risk of bias, across each of the following domains: random sequence generation; allocation concealment; blinding of participants and personnel; blinding of outcome assessment; incomplete outcome data; selective outcome reporting; and other potential sources of bias. Both review authors resolved any disagreement by discussion or by involving a third review author.

\section{Overall risk of bias}

We summarised the overall risk of bias at two levels: within studies (across domains), and across studies using the GRADE approach (Balshem 2010, GRADEpro GDT 2015). The GRADE findings are summarised in Summary of findings for the main comparison, Summary of findings 2 and Summary of findings 3.

We assessed the likely magnitude and direction of bias in each of the above-mentioned domains, if they were likely to impact the findings. We considered studies at high risk of overall bias if they were at high risk of bias for sequence generation, allocation concealment and blinding of outcome assessment. We considered studies at low risk of bias if they were at low risk of bias for sequence generation, allocation concealment and blinding of outcome assessment. We explored the impact of the level of bias through a Sensitivity analysis.

\section{Measures of treatment effect}

\section{Dichotomous data}

For dichotomous data, we presented results as a risk ratio (RR) with $95 \%$ confidence intervals $(\mathrm{Cl})$. 


\section{Continuous data}

For continuous data, we used the mean difference (MD) with $95 \%$ $\mathrm{Cl}$ if outcomes were measured in the same way between studies. We used the standardised mean difference (SMD) with $95 \% \mathrm{Cl}$ to combine studies that measured the same outcome but used different measurement methods.

When some studies reported endpoint data and others reported change from baseline data (with errors), we combined these in the meta-analysis if the outcomes were reported using the same scale.

Please refer to our protocol, Das 2017, and Table 1 for methodology for rate data, archived for use in future updates of this review.

\section{Unit of analysis issues}

\section{Cluster-randomised trials}

All included cluster-randomised trials reported cluster-adjusted estimates. We combined the results from the individuallyrandomised and cluster-randomised trials. We considered it reasonable to combine the results from both when there was little heterogeneity between the study designs and the interaction between the effect of intervention and the choice of randomisation unit was considered unlikely.

\section{Trials with more than two treatment groups}

For trials with more than two intervention groups (multi-arm trials), we included the directly relevant arms only. If we identified trials with various relevant arms, we combined the groups to form a single pair-wise comparison (Higgins 2011b), and included the disaggregated data in the corresponding subgroup category. If the control group was shared by two or more study arms, we divided the control group (events and total population) over the number of relevant subgroup categories to avoid double counting the participants. We noted the details of all the intervention and control arms in the Characteristics of included studies tables.

\section{Dealing with missing data}

We attempted to obtain missing data from the study investigators. If this was not possible, we reported the data as missing and did not attempt to impute values.

We described missing data, including dropouts (attrition), in the 'Risk of bias' tables. Differential dropout rates can lead to biased estimates of the effect size, and bias may arise if the reasons for dropping out differ across groups. We reported the reasons for dropout, where available. If data were missing for some cases, or if the reasons for dropping out were not reported, we contacted the study authors and documented if the authors could not be contacted or did not respond.

For all outcomes, we carried out analyses, as far as possible, on an intention-to-treat basis (i.e. we attempted to include all participants randomised to each group in the analyses, and all participants were analysed in the group to which they were allocated, regardless of whether or not they received the allocated intervention). The denominator for each outcome in each trial was the number randomised minus any participants whose outcomes were known to be missing.

\section{Assessment of heterogeneity}

We assessed methodological heterogeneity by examining the methodological characteristics and risk of bias of the included trials, and clinical heterogeneity by examining the similarity between the types of participants, interventions and outcomes.

For statistical heterogeneity, we examined the forest plots from meta-analyses to look for heterogeneity among trials, and used the $1^{2}$ statistic, Tau $^{2}$ and $\mathrm{Chi}^{2}$ test to quantify the level of heterogeneity among the trials in each analysis. If we identified moderate or substantial heterogeneity, we explored it by pre-specified subgroup analysis (see Subgroup analysis and investigation of heterogeneity).

We advise caution in the interpretation of analyses with high degrees of heterogeneity $\left(\mathrm{I}^{2}>50 \%\right)$. Where applicable, we have downgraded the quality of the evidence based on heterogeneity.

\section{Assessment of reporting biases}

We did not find 10 or more studies reporting on a single similar outcome, hence we could not assess for reporting bias. For the methodology to assess reporting bias in future updates of this review, please refer to our protocol, Das 2017, and Table 1.

\section{Data synthesis}

We carried out statistical analysis using RevMan 5 (Review Manager 2014). We used a random-effects model as our primary analysis for combining data, considering the differences in the intervention, and tested the robustness of this decision by re-analysing the data using a fixed-effect model and comparing the results (see Sensitivity analysis). We used the generic inverse-variance method for analyses that included cluster-randomised trials; for those that did not, we used the Mantel-Haenszel method.

In the Effects of interventions section, we list the primary outcomes for each comparison with estimates of relative effects, along with the number of participants and trials contributing data for each outcome. We present results as the average treatment effect with $95 \% \mathrm{Cls}$, and estimates of $\mathrm{I}^{2}$ (Deeks 2011).

We treated the random-effects summary as the average range of possible treatment effects and we discussed the clinical implications of treatment effects differing between trials in the Discussion section (Summary of main results; Overall completeness and applicability of evidence).

\section{'Summary of findings'}

For the assessment across included trials, we set out the main findings of the review in 'Summary of findings' tables, prepared using GRADE software (GRADEpro GDT 2015). For each comparison (as mentioned in the Types of interventions section), we listed the primary outcomes (stunting, wasting, underweight, anaemia, adverse effects), assessed at 12, 18 and/or 24 months of age, with estimates of relative effects, along with the number of participants and trials contributing data for each outcome.

For each individual outcome, we assessed the quality of the evidence using the GRADE approach (Balshem 2010). This involves consideration of within-study risk of bias (methodological quality), directness of evidence, heterogeneity, precision of effect estimates 
and risk of publication bias, and results in one of four quality ratings (high, moderate, low or very low).

\section{Subgroup analysis and investigation of heterogeneity}

We conducted the following exploratory subgroup analyses on the primary outcomes (where possible), irrespective of heterogeneity, and when there were more than three studies contributing data.

1. Energy content/formulation of product provided (SQ LNS providing 110 to $120 \mathrm{kcal}$ per day; MQ LNS providing 250 to 280 kcal per day; LQ LNS providing $>280$ kcal per day)

2. Duration of the intervention (up to six months versus six to 12 months versus more than 12 months duration)

3. Age at follow-up (at 12 months versus at 18 months versus at 24 months versus at 36 months of age)

We assessed differences between subgroups by inspection of the subgroups' $\mathrm{Cl}$; a non-overlapping $\mathrm{Cl}$ indicated a statistically significant difference in treatment effect between the subgroups. We also applied interaction tests, when possible.

\section{Sensitivity analysis}

We carried out sensitivity analyses to determine:
1. the effects of removing trials at high risk of bias (trials with poor or unclear allocation concealment and either blinding or high or imbalanced loss to follow-up) from the analysis;

2. the effects of removing non-randomised studies from the analysis;

3. the robustness of the results when using a fixed-effect model;

4. the effects of different intraclass correlation coefficients (ICCS) for cluster-randomised trials (where these were included); and

5. the effects of removing trials that also supplemented pregnant women with LNS in addition to children.

\section{RES U LTS}

\section{Description of studies}

\section{Results of the search}

Our searches identified a total of 14,390 potentially relevant titles from the electronic searches and 67 records from searching other sources . After removing duplicates, we screened 8124 records for eligibility and excluded 8036 on the basis of title and abstract. We obtained the full-text reports of the remaining 88 records, and of these, excluded 29 reports (26 studies) and included 54 reports (17 studies) in the review. We also identified five ongoing studies. Figure 1 depicts the search flow diagram. 
Figure 1. Study flow diagram.

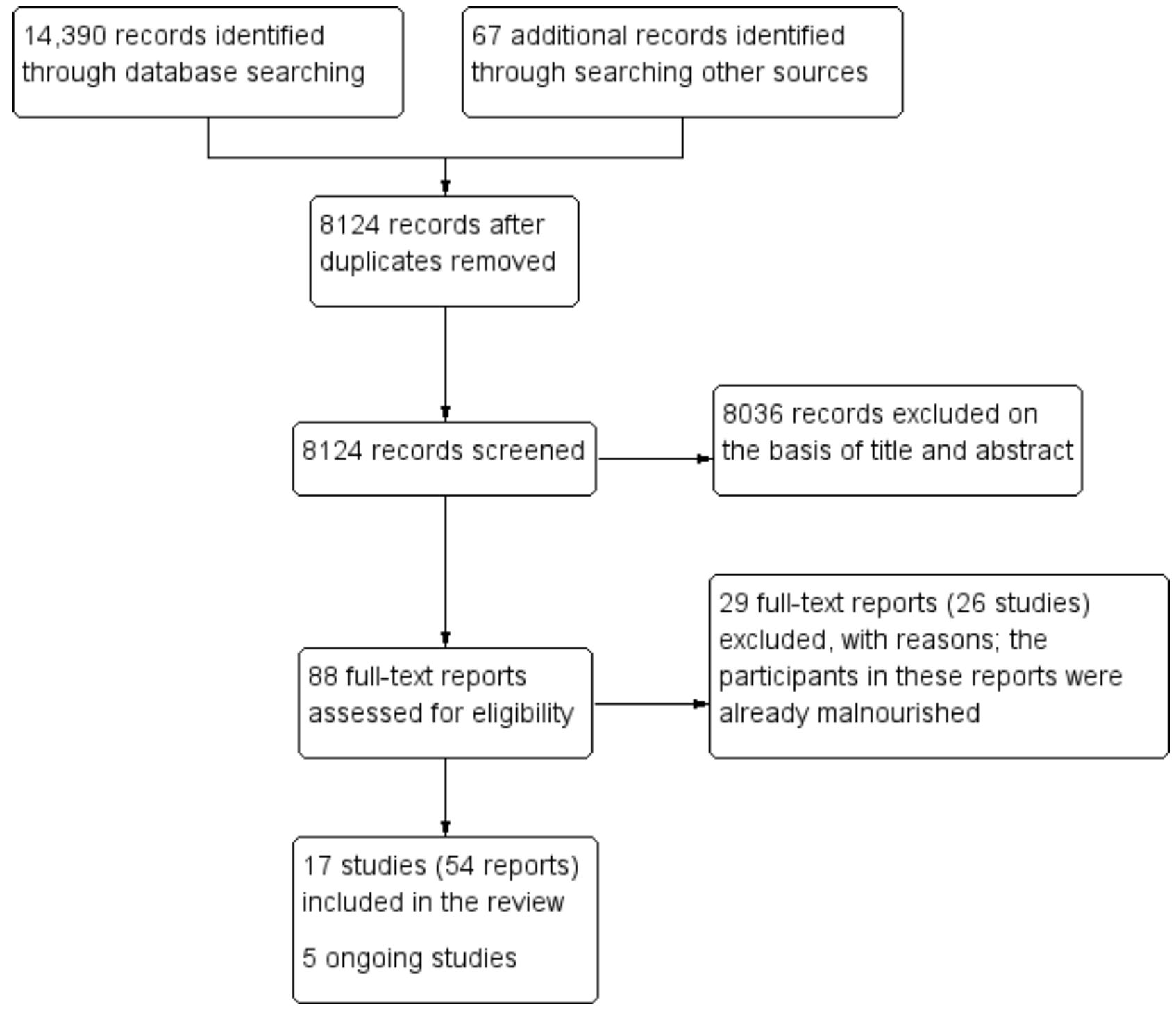

\section{Included studies}

This review includes 17 studies (from 54 reports) with 23,200 children (Adu-Afarwuah 2007; Adu-Afarwuah 2016; Ashorn 2015; Bisimwa 2012; Christian 2015; Dewey 2017; Hess 2015; Huybregts 2012; Iannotti 2014; Kumwenda 2014; Luby 2018; Mangani 2015; Matias 2017; Null 2018; Olney 2018; Phuka 2008; Siega-Riz 2014). All included studies were randomised controlled trials (RCTs) and published after the year 2007. See Characteristics of included studies tables.

\section{Settings}

Four studies were conducted in Malawi (Ashorn 2015; Kumwenda 2014; Mangani 2015; Phuka 2008), three in Bangladesh (Christian 2015; Dewey 2017; Luby 2018), two in Ghana (Adu-Afarwuah 2007; Adu-Afarwuah 2016), and one each in Burkina Faso (Hess 2015), Chad (Huybregts 2012), Congo (Bisimwa 2012), Guatemala (Olney 2018), Haiti (lannotti 2014), Honduras (Siega-Riz 2014), Kenya (Null 2018) and Peru (Matias 2017).
None of the included studies were conducted in emergency settings.

\section{Participants}

Most studies included children aged six months to 18 months (Adu-Afarwuah 2007; Adu-Afarwuah 2016; Ashorn 2015; Bisimwa 2012; Christian 2015; Hess 2015; Iannotti 2014; Kumwenda 2014; Mangani 2015; Matias 2017; Phuka 2008; Siega-Riz 2014). Four studies included children aged six to 24 months (Dewey 2017; Olney 2018; Luby 2018; Null 2018) and one study included children aged six to 36 months (Huybregts 2012).

Four included studies enrolled pregnant women and provided lipid-based nutrient supplements (LNS) plus complementary feeding during pregnancy and post-partum, followed by infant supplementation at six months of age (Adu-Afarwuah 2016; Ashorn 2015; Dewey 2017; Olney 2018). However, Dewey 2017 had an intervention arm in which only children were supplemented, hence we only used the data from that arm in the analysis in this review. 
The other studies provided LNS plus complementary feeding to children after six months of age.

\section{Interventions}

Ten studies provided small quantity lipid-based nutrient supplements (SQ LNS) (Adu-Afarwuah 2007; Adu-Afarwuah 2016; Ashorn 2015; Dewey 2017; Hess 2015; Iannotti 2014; Luby 2018; Matias 2017; Null 2018; Olney 2018). Four studies provided MQ LNS (Bisimwa 2012; Huybregts 2012; Mangani 2015; Siega-Riz 2014). Two studies provided both SQ LNS and MQ LNS (Kumwenda 2014; Phuka 2008), and one study provided SQ LNS to children aged six to 12 months and MQ LNS to children aged 12 to 18 months (Christian 2015). Table 2 provides further details on the composition of the LNS used in the included studies.

Five studies provided other co-interventions along with the LNS: Christian 2015 provided nutrition education and counselling; Hess 2015 provided malaria and diarrhoea treatment; Huybregts 2012 and Olney 2018 provided general food distribution; and Siega-Riz 2014 provided food vouchers and nutrition education.

The duration of the intervention ranged from a minimum of three months (lannotti 2014) to a maximum of 18 months (Dewey 2017; Luby 2018; Null 2018; Olney 2018).

\section{Comparisons}

Five of the 17 studies contributed data into multiple comparison groups (Adu-Afarwuah 2007; Christian 2015; Dewey 2017; Mangani 2015; Olney 2018)

Thirteen studies compared LNS plus complementary feeding with no intervention (Adu-Afarwuah 2007; Adu-Afarwuah 2016; Ashorn 2015; Christian 2015; Dewey 2017; Hess 2015; Huybregts 2012; Iannotti 2014; Kumwenda 2014; Luby 2018; Mangani 2015; Null 2018; Siega-Riz 2014). Four studies compared LNS plus complementary feeding with micronutrient powders (MNP) (AduAfarwuah 2007; Dewey 2017; Matias 2017; Olney 2018). Five studies compared LNS plus complementary feeding with fortified blended foods (FBF), including corn soy blend and UNIMIX (Bisimwa 2012; Christian 2015; Mangani 2015; Olney 2018; Phuka 2008).

We did not find any study comparing LNS plus complementary feeding with nutritional counselling.

\section{Outcome}

One or more of the pre-specified primary and secondary outcomes were reported by the studies included in the review.

Among the primary outcomes, nine studies reported stunting and wasting (Adu-Afarwuah 2016; Ashorn 2015; Christian 2015; Dewey 2017; Hess 2015; Kumwenda 2014; Luby 2018; Mangani 2015; Null 2018); eight studies reported underweight (Adu-Afarwuah 2016; Ashorn 2015; Christian 2015; Dewey 2017; Hess 2015; Kumwenda 2014; Luby 2018; Null 2018); five studies reported anaemia (AduAfarwuah 2007; Dewey 2017; Hess 2015; Huybregts 2012; Siega-Riz 2014), and three studies reported adverse events (Adu-Afarwuah 2016; Ashorn 2015; Kumwenda 2014). Although 11 studies reported on psychomotor and/or neuro-developmental outcomes (AduAfarwuah 2007; Adu-Afarwuah 2016; Ashorn 2015; Dewey 2017; Hess 2015; Kumwenda 2014; Luby 2018; Mangani 2015; Matias 2017; Null 2018; Phuka 2008), we were unable to combine the data from these studies in meta-analyses, due to variances in the comparisons and reported outcomes.

Among the secondary outcomes, six studies reported mid-upper arm circumference (MUAC) (Adu-Afarwuah 2016; Ashorn 2015; Dewey 2017; Hess 2015; Huybregts 2012; Kumwenda 2014); four studies reported haemoglobin levels (Adu-Afarwuah 2007; Hess 2015; Huybregts 2012; Siega-Riz 2014); Seven studies reported on morbidity (Christian 2015; Hess 2015; Huybregts 2012; Kumwenda 2014; Mangani 2015; Luby 2018; Null 2018); however, we were unable to combine the data from these studies in meta-analyses, due to variances in the comparisons and reported outcomes; and three studies reported mortality (Adu-Afarwuah 2016; Ashorn 2015; Kumwenda 2014). Twelve studies also reported on our explanatory secondary outcomes of height-for-age $z$ score (HAZ), weight-forage $z$ score (WAZ) and weight-for-height/length z score (WHZ) (AduAfarwuah 2007; Adu-Afarwuah 2016; Ashorn 2015; Christian 2015; Dewey 2017; Hess 2015; Huybregts 2012; Iannotti 2014; Kumwenda 2014; Luby 2018; Mangani 2015; Null 2018).

Most studies ( $\mathrm{n}=14$ ) measured outcomes at 12 months (nine studies: Adu-Afarwuah 2007; Bisimwa 2012; Iannotti 2014; Kumwenda 2014; Luby 2018; Matias 2017; Null 2018; Phuka 2008; Siega-Riz 2014), and 18 months of age (five studies: Adu-Afarwuah 2016; Ashorn 2015; Christian 2015; Hess 2015; Mangani 2015). Four studies measured outcomes at 24 months of age (Dewey 2017; Luby 2018; Null 2018; Olney 2018).

\section{Funding sources}

Most studies ( $n=13$ ) were funded non-commercially, through not-for-profit organisations, university grants and ministries (AduAfarwuah 2016; Ashorn 2015; Bisimwa 2012; Dewey 2017; Hess 2015; Huybregts 2012; Iannotti 2014; Kumwenda 2014; Luby 2018; Mangani 2015; Null 2018; Olney 2018; Siega-Riz 2014). A further two studies received funds from non-commercial entities but the LNS was provided by commercial entities (Christian 2015; Matias 2017). Two studies, Adu-Afarwuah 2007 and Phuka 2008, were funded by commercial entity.

Further details on funding sources and the specific funding agencies are specified in the Characteristics of included studies tables.

\section{Excluded studies}

We excluded 26 studies (from 29 reports) (Ackatia-Armah 2015; Adams 2017; Adams 2018; Ahmed 2014; Arimond 2017; Cercamondi 2013; Defourney 2009; Flax 2010; Flax 2013; Heidkamp 2012; Isanaka 2009; Iuel-Brockdorf 2015; Kuusipalo 2006; LaGrone 2012; Langendorf 2014; Maleta 2004; Maryam 2015; Muslihah 2016; Rantesalu 2017; Schlossman 2017; Style 2017; Thakwalakwa 2010; Thakwalakwa 2012; Thakwalakwa 2015; Unger 2017; VargasVásquez 2015). We excluded most studies because participants were malnourished at the time of recruitment. The key reasons for exclusion of studies are described in the Characteristics of excluded studies tables.

\section{Ongoing studies}

We included five ongoing studies (Borg 2017; Fernald 2016; Huybregts 2017; ISRCTN94319790; SHINE trial 2015), which could potentially be included in the future update of this review. All five studies are RCTs. 
1. The Borg 2017 study compares LNS with corn soy blend plus $(\mathrm{CSB}++)$, Sprinkles or no intervention. The main outcome of interest is anthropometric status (i.e. HAZ, WHZ and WAZ).

2. The MAHAY study (Fernald 2016) is a multi-arm trial comparing: an existing program with monthly growth monitoring and nutritional/hygiene education; home visits for intensive nutrition counselling within a behavior change framework; LNS for children aged six to 18 months; LNS supplementation of pregnant/lactating women; and an intensive home visiting program to support child development. The primary outcomes include growth and child development (mental, motor and social development), while the secondary outcomes include caregiver-reported child morbidity, household food security and diet diversity, micro-nutrient status, maternal knowledge of child care and feeding practices, and home stimulation practices.

3. The Prevention of Childhood Malnutrition (PROMIS) multicountry study evaluates the prevention (through age-stratified behaviour change communication targeted to the caregivers of the beneficiary children and the distribution of preventive doses of SQ LNS), screening, and referral of cases of acute malnutrition (Huybregts 2017). The primary outcomes include the incidence of acute malnutrition, monthly acute malnutrition screening coverage, and acute malnutrition treatment compliance.
4. The ISRCTN94319790 study is a community-based, clustercontrolled trial to evaluate the effectiveness of LNS and the proportional contribution of multisectoral interventions in the prevention of stunting among children under five years of age in Kurram Agency, Pakistan.

5. The Sanitation Hygiene Infant Nutrition Efficacy study is a $2 \times 2$ factorial, cluster-randomised, community-based trial conducted in two rural districts of Zimbabwe (SHINE trial 2015). The study comprises of four arms: water, sanitation, and hygiene (WASH) intervention; infant and young child feeding (IYCF) intervention; sanitation/hygiene and nutrition (WASH + IYCF); and standard care. The primary outcomes are HAZ and haemoglobin level at 18 months of age.

For further detail, see Characteristics of ongoing studies tables.

\section{Risk of bias in included studies}

A graphical summary of the results of the 'Risk of bias' assessment is provided in Figure 2 and Figure 3. Overall, most trials were at low risk of bias for random sequence generation, allocation concealment, blinding of outcome assessment, incomplete outcome data, selective reporting and other sources of bias. We considered most trials to be at high risk of bias for blinding of participants and personnel due to the nature of the intervention.

Figure 2. 'Risk of bias' graph: review authors' judgements about each risk of bias item presented as percentages across all included studies.

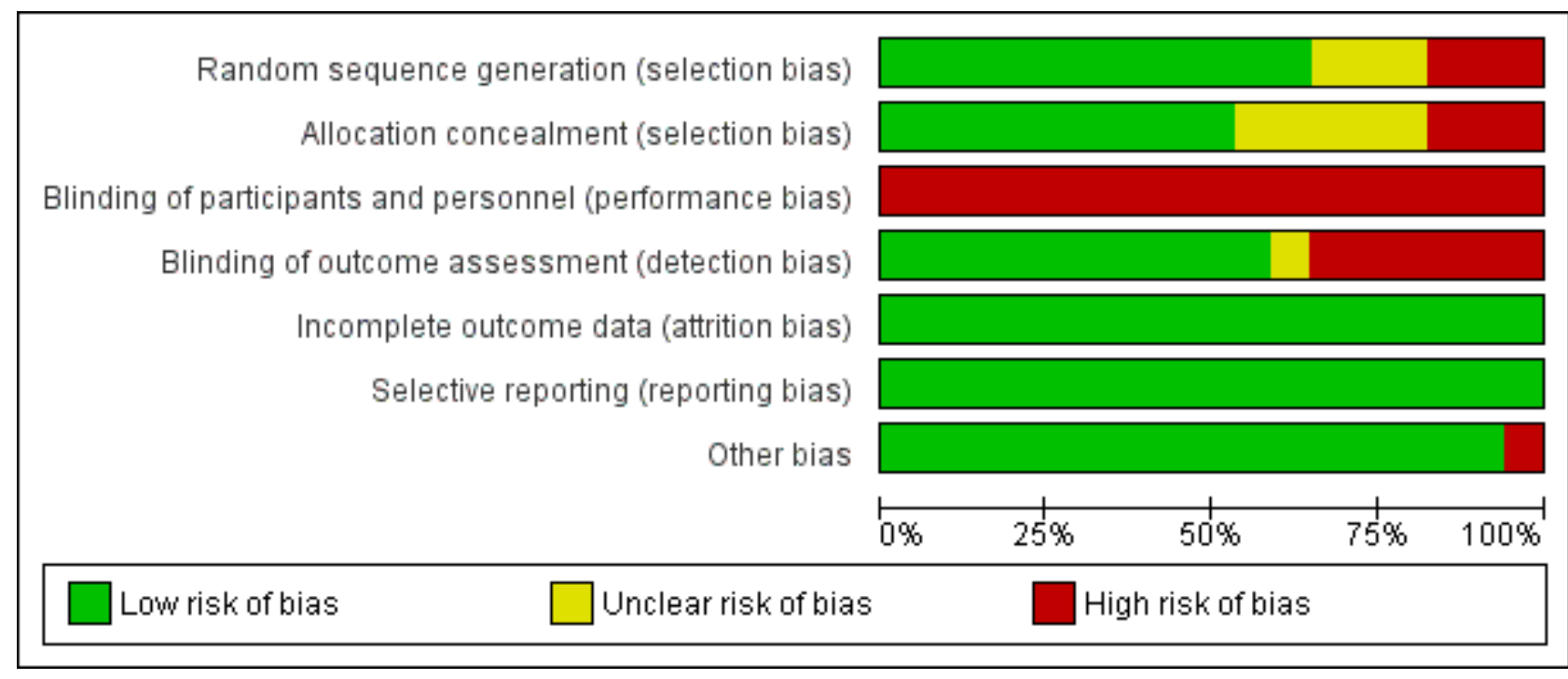


Figure 3. 'Risk of bias' summary: review authors' judgements about each risk of bias item for each included study.

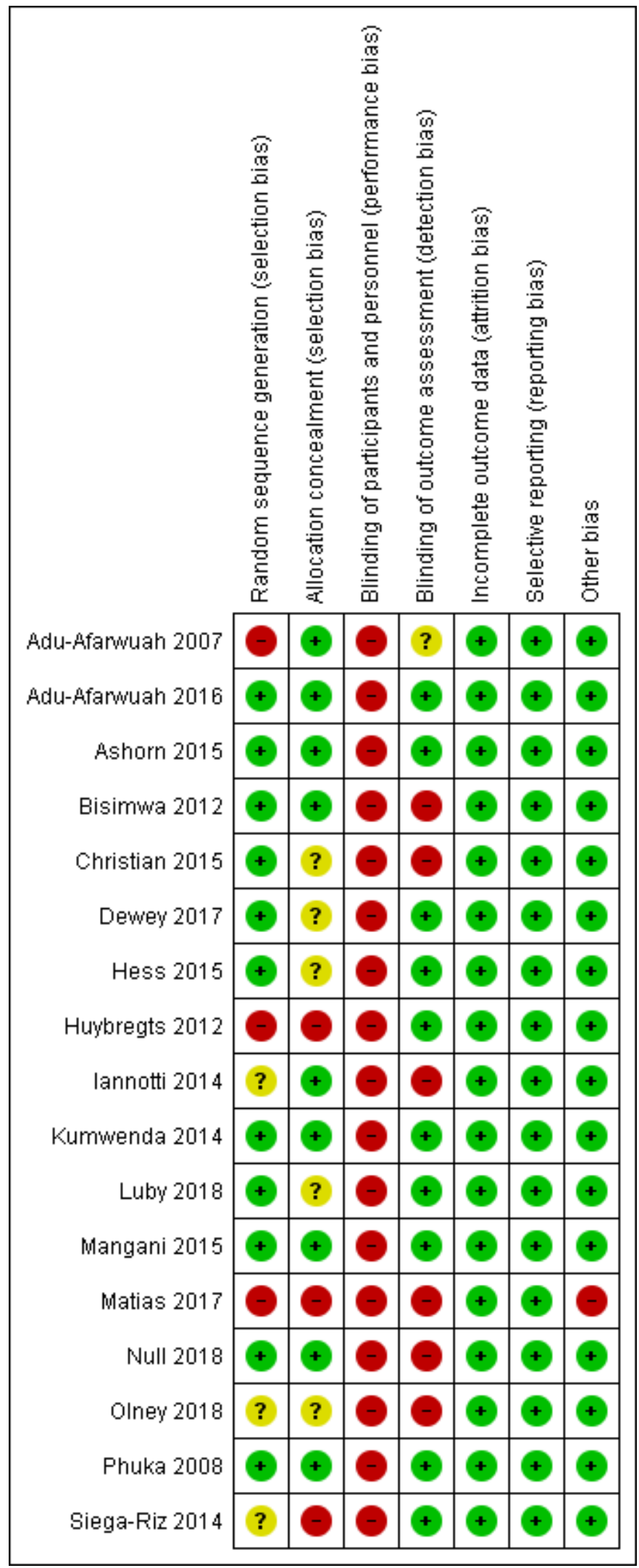

Preventive lipid-based nutrient supplements given with complementary foods to infants and young children 6 to 23 months of age for 


\section{Allocation}

\section{Random sequence generation}

For this domain, we rated three studies at high risk of bias since the methods used to sequence generation were not adequate (AduAfarwuah 2007; Huybregts 2012; Matias 2017); three studies at unclear risk of bias since they did not specify the actual methods used for random sequence generation (Iannotti 2014; Olney 2018; Siega-Riz 2014), and the remaining 11 studies at low risk of bias as they used adequate mechanisms for random sequence generation (Adu-Afarwuah 2016; Ashorn 2015; Bisimwa 2012; Christian 2015; Dewey 2017; Hess 2015; Kumwenda 2014; Luby 2018; Mangani 2015; Null 2018; Phuka 2008).

\section{Allocation concealment}

Three studies did not conceal the allocation so we rated them at high risk of bias (Huybregts 2012; Matias 2017; Siega-Riz 2014). We rated a further five studies at unclear risk of bias since they did not specify clearly the methods for allocation concealment (Christian 2015; Dewey 2017; Hess 2015; Luby 2018; Olney 2018). We considered the remaining nine trials to be at low risk of bias since the allocation was adequately concealed (Adu-Afarwuah 2007; Adu-Afarwuah 2016; Ashorn 2015; Bisimwa 2012; Iannotti 2014; Kumwenda 2014; Mangani 2015; Null 2018; Phuka 2008).

\section{Blinding}

\section{Blinding of participants and personnel}

We judged all 17 included studies to be at high risk of performance bias since participants and personnel were not blinded (AduAfarwuah 2007; Adu-Afarwuah 2016; Ashorn 2015; Bisimwa 2012; Christian 2015; Dewey 2017; Hess 2015; Huybregts 2012; lannotti 2014; Kumwenda 2014; Luby 2018; Mangani 2015; Matias 2017; Null 2018; Olney 2018; Phuka 2008; Siega-Riz 2014).

\section{Blinding of outcome assessment}

We rated 10 studies at low risk of detection bias as outcome assessors were adequately blinded to the allocated intervention (Adu-Afarwuah 2016; Ashorn 2015; Dewey 2017; Hess 2015; Huybregts 2012; Kumwenda 2014; Luby 2018; Mangani 2015; Phuka 2008; Siega-Riz 2014); six studies at high risk of detection bias as the outcome assessors were not blinded to the intervention allocation (Bisimwa 2012; Christian 2015; lannotti 2014; Matias 2017; Null 2018; Olney 2018), and one study at unclear risk of detection bias as it did not clearly specify whether the outcome assessor was blinded to the intervention allocation (Adu-Afarwuah 2007).

\section{Incomplete outcome data}

All included studies provided reasons for loss to follow-up.

We rated all 17 studies to be at low risk of attrition bias because they did not have high rates of loss to follow-up (Adu-Afarwuah 2007; Adu-Afarwuah 2016; Ashorn 2015; Bisimwa 2012; Christian 2015; Dewey 2017; Hess 2015; Huybregts 2012; Iannotti 2014; Kumwenda 2014; Luby 2018; Mangani 2015; Matias 2017; Null 2018; Olney 2018; Phuka 2008; Siega-Riz 2014).

\section{Selective reporting}

We looked for published protocols and trial registration records to assess for selective reporting. With the exception of one study (Christian 2015), we found published protocols or trial registration records for all included studies. We judged all 17 included studies to be at low risk of reporting bias since the outcomes prespecified in the protocols were reported (Adu-Afarwuah 2007; AduAfarwuah 2016; Ashorn 2015; Bisimwa 2012; Dewey 2017; Hess 2015; Huybregts 2012; Iannotti 2014; Kumwenda 2014; Luby 2018; Mangani 2015; Matias 2017; Null 2018; Olney 2018; Phuka 2008; Siega-Riz 2014). For Christian 2015, although we did not find the published protocol or trial registration record, the outcomes specified in the methodology section were reported in the paper. Hence, we judged Christian 2015 at low risk of reporting bias also.

\section{Other potential sources of bias}

We judged one study, Matias 2017, at high risk of other bias since it specified that the supplement was unavailable for a period of two months during the study, and during that time the probability of being enrolled in each group differed, but that those already enrolled in the LNS group continued receiving their monthly LNS supply. None of the other included studies reported any other potential sources of bias, so we rated those at low risk of other bias (Adu-Afarwuah 2007; Adu-Afarwuah 2016; Ashorn 2015; Bisimwa 2012; Christian 2015; Dewey 2017; Hess 2015; Huybregts 2012; lannotti 2014; Kumwenda 2014; Luby 2018; Mangani 2015; Null 2018; Olney 2018; Phuka 2008; Siega-Riz 2014).

\section{Effects of interventions}

See: Summary of findings for the main comparison; Summary of findings 2; Summary of findings 3

\section{Comparison 1: LNS versus no intervention}

Thirteen studies compared LNS plus complementary feeding with no intervention (Adu-Afarwuah 2007; Adu-Afarwuah 2016; Ashorn 2015; Christian 2015; Dewey 2017; Hess 2015; Huybregts 2012; Iannotti 2014; Kumwenda 2014; Luby 2018; Mangani 2015; Null 2018; Siega-Riz 2014). The included studies reported on one or more of the primary outcomes.

We rated the quality of the evidence for all outcomes as low or moderate, as per GRADE criteria. We downgraded the quality of the evidence due to study limitations, inconsistency and imprecision. See Summary of findings for the main comparison.

\section{Primary outcomes}

\section{Stunting: pooled study results}

Compared to no intervention, LNS plus complementary feeding reduced the prevalence of moderate stunting by $7 \%$ (risk ratio (RR) $0.93,95 \%$ confidence interval (Cl) 0.88 to 0.98 ; nine studies; 13 comparisons, 13,372 participants; $\mathrm{I}^{2}=25 \%$; $\mathrm{Tau}^{2}=0.00 ; \mathrm{Chi}^{2}=$ 15.90; moderate-quality evidence; Analysis 1.1; Figure 4) and severe stunting by $15 \%$ (RR $0.85,95 \% \mathrm{Cl} 0.74$ to 0.98 ; five studies (two of which contributed data to multiple comparisons; total number of comparisons = nine), 6151 participants, $\mathrm{I}^{2}=0 \%$; $\mathrm{Tau}^{2}=0.00 ; \mathrm{Chi}^{2}=$ 3.70; moderate-quality evidence; Analysis 1.2). 
Figure 4. Forest plot of comparison: 1 LNS versus no intervention, outcome: 1.1 Moderate stunting.

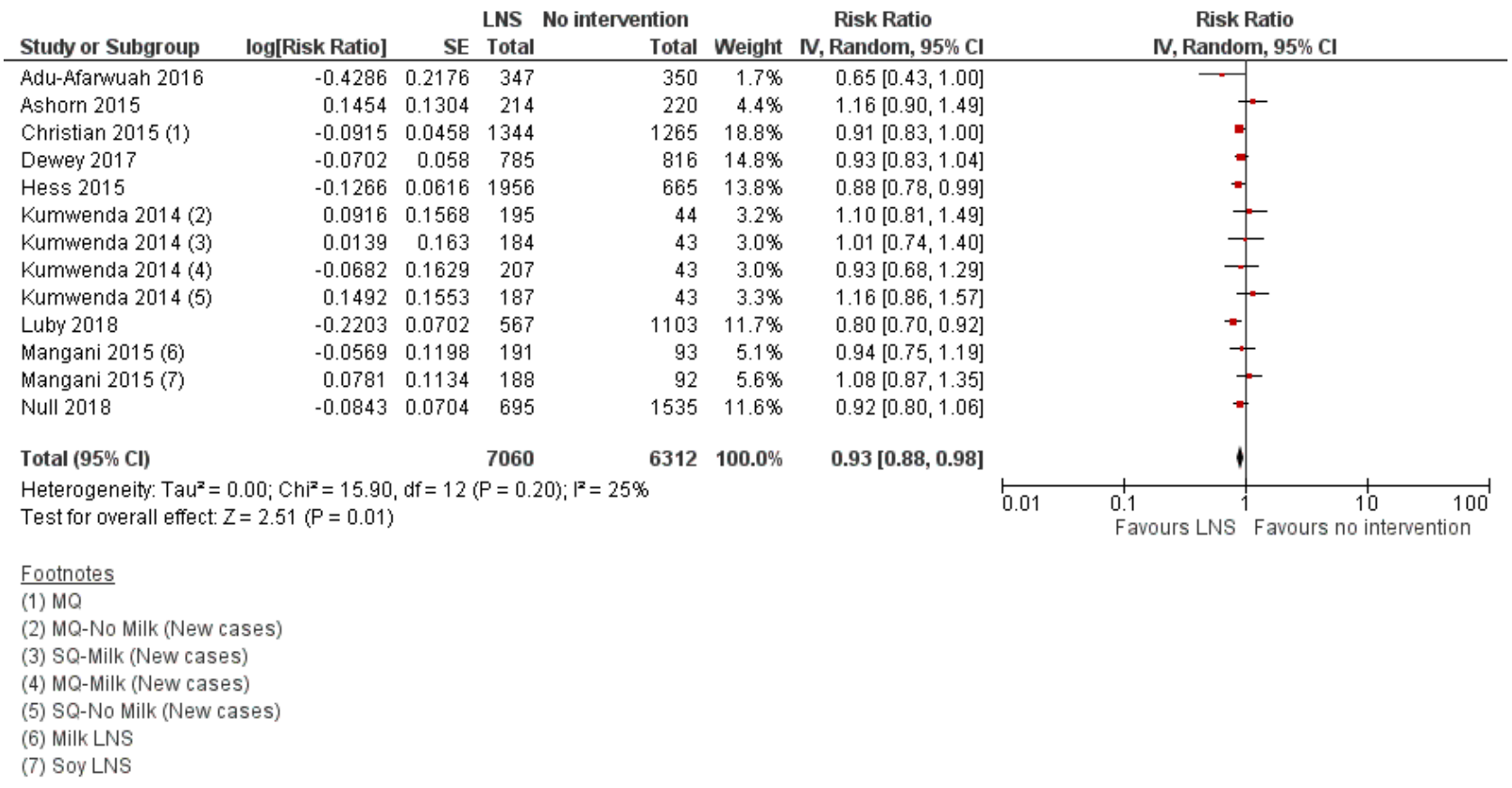

We conducted a sensitivity analysis by removing trials that also supplemented pregnant women with LNS in addition to children (Adu-Afarwuah 2016; Ashorn 2015), and found no significant difference in the outcome (Analysis 1.3; Analysis 1.4).

\section{Wasting: pooled study results}

Compared to no intervention, LNS plus complementary feeding reduced the prevalence of moderate wasting by $18 \%$ (RR $0.82,95 \%$
$\mathrm{Cl} 0.74$ to 0.91 ; eight studies; 11 comparisons), 13,172 participants; $\mathrm{I}^{2}=0 \% ; \mathrm{Tau}^{2}=0.00 ; \mathrm{Chi}^{2}=4.22 ;$ moderate-quality evidence; Analysis 1.5; Figure 5), but had no effect on the prevalence of severe wasting (RR $1.27,95 \% \mathrm{Cl} 0.66$ to 2.46 ; three studies (two of which contributed data to multiple comparisons; total number of comparisons = seven), 2329 participants; $\mathrm{I}^{2}=0 \% ; \mathrm{Tau}^{2}=0.00 ; \mathrm{Chi}^{2}$ = 4.52; moderate-quality evidence; Analysis 1.6).

Figure 5. Forest plot of comparison: 1 LNS versus no intervention, outcome: 1.5 Moderate wasting.

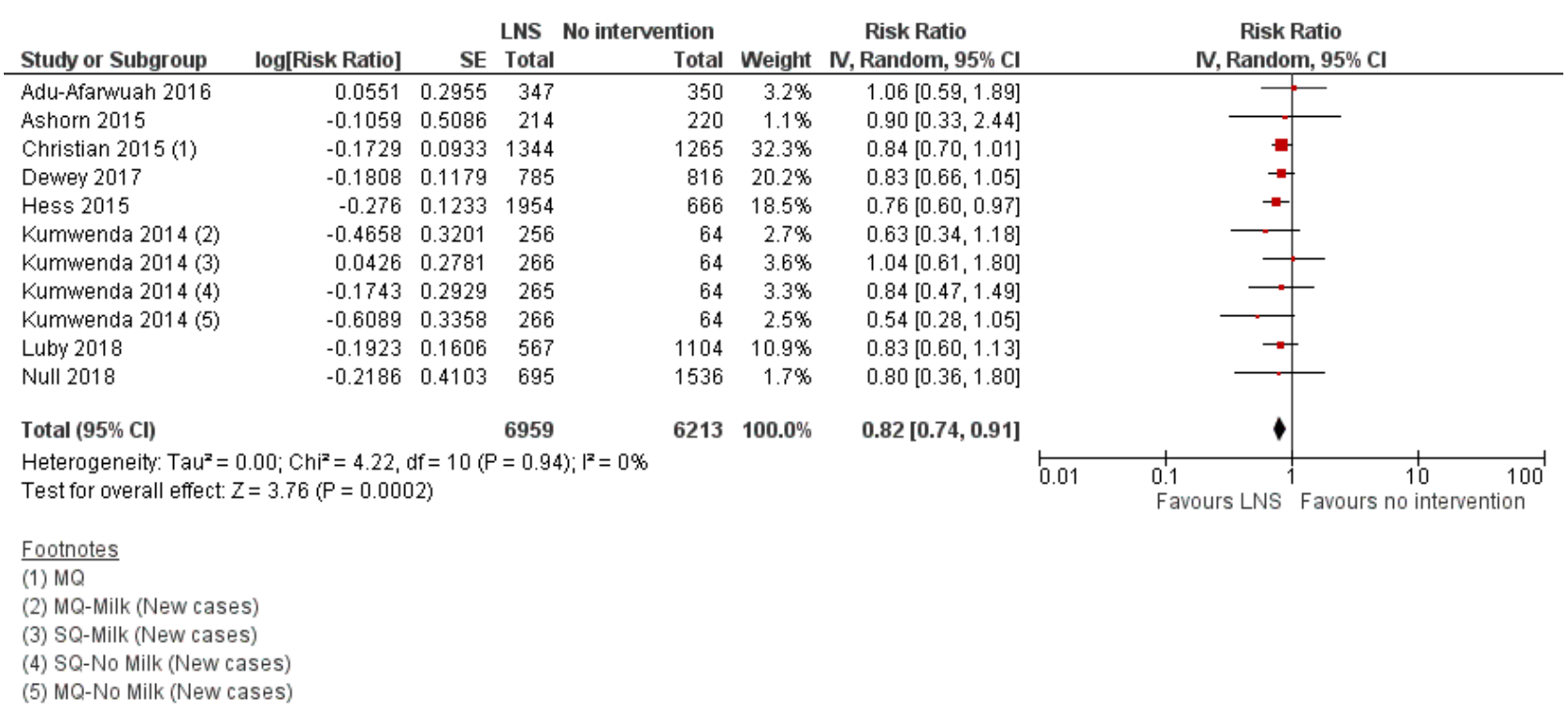

We conducted sensitivity analysis after removing trials that also supplemented pregnant women with LNS in addition to children
(Adu-Afarwuah 2016; Ashorn 2015), and found no significant difference in the outcome (Analysis 1.7; Analysis 1.8). 


\section{Underweight: pooled study results}

Compared to no intervention, LNS plus complementary feeding significantly reduced the prevalence of moderate underweight by $15 \%$ (RR $0.85,95 \% \mathrm{Cl} 0.80$ to 0.91 ; eight studies; 11 comparisons), 13,073 participants; $\mathrm{I}^{2}=0 \%$; $\mathrm{Tau}^{2}=0.00 ; \mathrm{Chi}^{2}=9.44$; moderatequality evidence; Analysis 1.9), but had no effect on the prevalence of severe underweight (RR $0.78,95 \% \mathrm{Cl} 0.54$ to 1.13 ; two studies (one of which contributed data to four comparisons; total number of comparisons = five), 1729 participants; $12=0 \%$; Tau $^{2}=0.00 ;$ Chi $^{2}$ $=0.97$; moderate-quality evidence; Analysis 1.10 ).

We conducted sensitivity analysis after removing trials that also supplemented pregnant women with LNS in addition to children (Adu-Afarwuah 2016; Ashorn 2015) and found no significant difference in the outcome (Analysis 1.11; Analysis 1.12).

\section{Anaemia: pooled study results}

Compared to no intervention, LNS plus complementary feeding significantly reduced anaemia by $21 \%(\mathrm{RR} 0.79,95 \% \mathrm{Cl} 0.69$ to 0.90 ; five studies, 2332 participants; $\mathrm{I}^{2}=66 \%$; $\mathrm{Tau}^{2}=0.01 ; \mathrm{Chi}^{2}=11.75$; low-quality evidence; Analysis 1.13).

\section{Psychomotor development and neuro-developmental outcomes: single study results}

Nine studies reported data on psychomotor and neurodevelopmental outcomes (Adu-Afarwuah 2007; Adu-Afarwuah 2016; Ashorn 2015; Dewey 2017; Hess 2015; Kumwenda 2014; Luby 2018; Mangani 2015; Null 2018). Most of the studies reported data on gross motor development; however, we were unable to combine these data in a meta-analysis due to variations in definitions and measurements. Hence, we have provided a narrative description of the findings for these outcomes directly below.

Adu-Afarwuah 2007 reported that children in the LNS plus complementary feeding group "walked independently at 12 months" significantly earlier than the children in the nointervention group (RR $3.4,95 \% \mathrm{Cl} 1.67$ to 6.43 ), while there was no difference between the groups for "standing alone at 12 months".

Adu-Afarwuah 2016 reported a significantly higher proportion of children "walking alone" in the LNS plus complementary feeding group at 12 months of age than in the no-intervention group (RR $1.23,95 \% \mathrm{Cl} 1.02$ to 1.49 ). However, there were no differences between the groups for the other motor, language, socio-emotional and executive function outcomes at 18 months of age.

Ashorn 2015 reported that children in the LNS plus complementary feeding group "walked alone" (B (beta) $0.53,95 \% \mathrm{Cl} 0.11$ to 0.94 ) and "waved goodbye" (B $0.60,95 \% \mathrm{Cl} 0.12$ to 1.08 ) earlier than children in the no-intervention group. At 12 months age, more children in the LNS plus complementary feeding group "walked alone" than in the no-intervention group. There were no differences between the groups for any of the other outcomes, including motor, language, socio-emotional and executive function skills, at 18 months of age.

Dewey 2017 reported improved motor development (using the Developmental Milestones Checklist II (DMC-II) with minor adaptations) in the LNS plus complementary feeding group compared to the no intervention group at 18 and 24 months of age. Receptive language scores were also reported to be higher for the LNS plus complementary feeding group at 18 and 24 months of age. There were no differences between the groups for expressive language scores (using a vocabulary inventory based on the principles of the MacArthur Communicative Development Inventories (CDI), personal-social development scores (assessed by DMC-II with minor adaptations) and executive function scores (with the use of the A-not-B task) at 18 or 24 months of age.

Hess 2015 reported that children in the LNS plus complementary feeding group had better motor (mean difference (MD) 0.34 points, $95 \% \mathrm{Cl} 0.21$ to 0.46 ), language (MD 0.30 points, $95 \% \mathrm{Cl} 0.15$ to 0.44 ), and personal social development scores (MD 0.32 points, $95 \%$ $\mathrm{Cl} 0.16$ to 0.48 ) than children in the no-intervention group at 18 months of age (all assessed by DMC-II), with no impact on caregiverchild interaction.

Kumwenda 2014 and Mangani 2015 did not find any significant impact of LNS plus complementary feeding on any of the psychomotor and neuro-developmental outcomes; including motor development, language development, socio-emotional development and executive function development measured at 18 months of age.

Luby 2018 reported improved motor milestone attainment for 'walking alone' in the nutrition group compared to the control group after one year of intervention (hazard ratio (HR): $1 \cdot 32 ; 95 \%$ $\mathrm{Cl} 1.07$ to 1.62). After two years of intervention, nutrition group had improved communication Z score (MD: $0 \cdot 19 ; 95 \% \mathrm{Cl} 0 \cdot 10$ to $0 \cdot 28$ ); gross motor Z score (MD: 0.19; $95 \%$ Cl 0.08 to 0.30); personal social Z score (MD: 0.22; 95\% Cl: 0.11 to 0.33); combined Z score (MD: 0.28; $95 \% \mathrm{Cl} 0 \cdot 18$ to $0 \cdot 37$ ); comprehension score (MD: 0.19; $95 \% \mathrm{Cl} 0.08$ to $0 \cdot 29$ ); and expressive language score (MD: $0.18 ; 95 \% \mathrm{Cl} 0.07$ to $0 \cdot 29)$ compared to the control group. There was no effect on any other motor milestone attainment after one year of intervention and executive function scores after two years of intervention.

Null 2018 reported no impact on any of the motor milestone attainment, communication, gross motor, personal social, and combined scales of the Extended Ages and Stages

Questionnaire in the nutrition group compared to the control group.

\section{Any adverse effects: pooled study results}

The authors of the three studies reporting on adverse effects defined adverse effects as a cumulative number of serious adverse events that included deaths, hospitalisations, congenital abnormalities and life-threatening conditions requiring an immediate hospital visit (Adu-Afarwuah 2016; Ashorn 2015; Kumwenda 2014). We found no differences in adverse effects reported in the LNS plus complementary feeding group compared to the no-intervention group ( $\mathrm{RR} 0.86,95 \% \mathrm{Cl} 0.74$ to 1.01 ; three studies; 3382 participants; moderate-quality evidence; $12=30 \%$; $\mathrm{Tau}^{2}=0.01 ; \mathrm{Chi}^{2}=4.30 ;$ Analysis 1.14).

We conducted sensitivity analysis after removing trials that also supplemented pregnant women with LNS in addition to children (Adu-Afarwuah 2016; Ashorn 2015), and found significantly reduced adverse events in the LNS group plus complementary feeding group compared to the no-intervention group (RR 0.76, 95\% Cl 0.60 to 0.95 ; one study (two comparisons; total number of comparisons = 2); 1772 participants; $\mathrm{I}^{2}=17 \%$; $\mathrm{Tau}^{2}=0.00 ; \mathrm{Chi}^{2}=1.21$; Analysis 1.15).

None of the included trials reported allergies as an adverse effect. 


\section{Secondary outcomes}

\section{MUAC: pooled study results}

Compared to no intervention, LNS plus complementary feeding significantly improved MUAC (standardised mean difference (SMD) $0.13,95 \% \mathrm{Cl} 0.05$ to 0.22 ; six studies (one of which contributed data to four comparisons; total number of comparisons = nine), 8187 participants; $\mathrm{I}^{2}=70 \% ; \mathrm{Tau}^{2}=0.01 ; \mathrm{Chi}^{2}=26.47 ; \mathrm{I}^{2}=70 \%$; low-quality evidence; Analysis 1.16).

\section{Haemoglobin: pooled study results}

Compared to no intervention, LNS plus complementary feeding significantly improved serum haemoglobin (MD $5.78 \mathrm{~g} / \mathrm{L}, 95 \% \mathrm{Cl}$ 2.27 to 9.30; four studies, 4518 participants; $\mathrm{I}^{2}=96 \%$; $\mathrm{Tau}^{2}=11.66$; $\mathrm{Chi}^{2}=78.77$; low-quality evidence; Analysis 1.17 ).

\section{Morbidity: single study results}

Seven studies reported on morbidity (Christian 2015; Hess 2015; Huybregts 2012; Kumwenda 2014; Mangani 2015; Luby 2018; Null 2018); however, we could not pool the outcomes in a meta-analysis, since they were not consistently reported across these seven studies.

Christian 2015 reported no differences in the incidence of pneumonia (RR $0.98,95 \% \mathrm{Cl} 0.80$ to1.20), diarrhoea (RR 0.97, 95\% $\mathrm{Cl} 0.85$ to 1.10 ) and diarrhoeal dysentery (RR $1.03,95 \% \mathrm{Cl} 0.90$ to $1.19)$ in the intervention and control groups.

Hess 2015 reported no differences in the incidence of diarrhoea ( $P=$ $0.59)$ and malaria $(P=0.88)$ in the intervention and control groups.

Huybregts 2012 reported decreased reported episodes of diarrhoea (incidence rate ratio $0.71,95 \% \mathrm{Cl} 0.63$ to 0.80 ) and fever (incidence rate ratio $0.77,95 \% \mathrm{Cl} 0.70$ to 0.86 ) in the LNS plus complementary feeding group compared to the no intervention group.

Kumwenda 2014 reported no differences between the intervention and control groups for guardian-reported illnesses (incidence rate ratio $1.05,95 \% \mathrm{Cl} 0.93$ to 1.19 ); however, malaria-related, nonscheduled visits were reported to be higher in the MQ LNS group (incidence rate ratio $1.21,95 \% \mathrm{Cl} 1.00$ to 1.46 ).

Mangani 2015 reported no differences between children in the LNS plus complementary feeding and no-intervention groups for malaria (incidence per child year of 0.51 in the LNS plus complementary feeding group compared to 0.64 in the nointervention group) and clinical pneumonia (incidence per child year of 0.91 in the LNS plus complementary feeding group compared to 0.96 in the no-intervention group).

Luby 2018 reported reduced prevalence of diarrhoea in the nutrition group compared to the control group (prevalence ratio: $0.64 ; 95 \% \mathrm{Cl} 0.49$ to 0.85 ).

Null 2018 reported no difference in diarrhoea prevalence between the nutrition and the control group (prevalence ratio: $0.96 ; 95 \% \mathrm{Cl}$ : 0.86 to 1.08$)$.

\section{Mortality: pooled study results}

Compared to no intervention, LNS plus complementary feeding did not have any impact on mortality ( RR $0.93,95 \% \mathrm{Cl} 0.63$ to 1.37 ; three studies (one of which contributed data to two comparisons; total number of comparisons = four), 3321 participants; $\left.\right|^{2}=10 \% ;$ Tau $^{2}=$ $0.02 ; \mathrm{Chi}^{2}=3.32$; moderate-quality evidence; Analysis 1.18).

\section{Explanatory secondary outcomes}

\section{HAZ: pooled study results}

Compared to no intervention, LNS plus complementary feeding improved HAZ (SMD $0.11,95 \% \mathrm{Cl} 0.05$ to $0.16 ; 12$ studies; 15,795 participants; $I^{2}=65 \% ;$ Tau $^{2}=0.01 ;$ Chi $^{2}=43.43 ;$ low-quality evidence; Analysis 1.19).

\section{WAZ: pooled study results}

Compared to no intervention, LNS plus complementary feeding improved WAZ (SMD 0.09, 95\% Cl 0.02 to $0.16 ; 10$ studies, 12,188 participants; $\mathrm{I}^{2}=65 \% ; \mathrm{Tau}^{2}=0.01 ; \mathrm{Chi}^{2}=37.11 ;$ low-quality evidence; Analysis 1.20).

\section{WHZ: pooled study results}

Compared to no intervention, LNS plus complementary feeding improved WHZ (SMD 0.08, 95\% Cl 0.04 to $0.13 ; 10$ studies, 12,894 participants; $\mathrm{I}^{2}=40 \% ; \mathrm{Tau}^{2}=0.00 ; \mathrm{Chi}^{2}=21.83$; moderate-quality evidence; Analysis 1.21).

\section{Subgroup analysis}

\section{Energy content/formulation of product provided}

None of the studies included in this comparison provided LQ LNS.

\section{Primary outcomes}

A subgroup analysis by energy content suggested a marginally statistically significant impact of SQ LNS on the prevalence of severe stunting (RR $0.83,95 \% \mathrm{Cl} 0.70$ to 0.99 ; four studies; 5 comparisons), 4956 participants), while the impact of MQ LNS was not significant (RR $0.88,95 \% \mathrm{Cl} 0.69$ to 1.12 ; two studies; 4 comparisons ), 1195 participants). See Analysis 2.1.

There were no differences between SQ LNS and MQ LNS for the other primary outcomes: moderate stunting (Analysis 2.2); moderate wasting (Analysis 2.3) and severe wasting (Analysis 2.4); moderate underweight (Analysis 2.5) and severe underweight (Analysis 2.6); anaemia (Analysis 2.7); and adverse effects (Analysis 2.8).

\section{Secondary outcomes}

A subgroup analysis by energy content suggested an impact of MQ LNS on MUAC (SMD $0.17,95 \% \mathrm{Cl} 0.08$ to 0.26 ; two studies; 3 comparisons); 1641 participants; Analysis 2.9), and a marginal impact for SQ LNS (SMD 0.12, 95\% Cl -0.00 to 0.24 ; five studies; 6 comparisons); 6546 participants; Analysis 2.9).

There were no differences between SQ LNS and MQ LNS for serum haemoglobin and mortality ( Analysis 2.10; Analysis 2.11).

\section{Explanatory secondary outcomes}

There was no difference between SQ LNS and MQ LNS on HAZ (Analysis 2.12) and WHZ (Analysis 2.13). There was a significant impact of SQ LNS on WAZ (SMD: $0.11,95 \% \mathrm{Cl}: 0.02$ to 0.19 ; nine studies; ten comparisons; 10,959 participants; Analysis 2.14) while the impact of MQ LNS was not significant (SMD: 0.05; 95\% Cl -0.04 to 0.14 ; two studies; four comparisons; 1229 participants; Analysis 2.14). 
Duration of intervention (up to six months; six to 12 months; more than 12 months)

\section{Primary outcomes}

A subgroup analysis by duration of intervention suggested that a duration of more than 12 months reduced the prevalence of moderate stunting ( $\mathrm{RR} 0.89,95 \% \mathrm{Cl} 0.81$ to 0.97 ; three studies, 5501 participants; Analysis 3.1) and severe stunting (RR 0.80, 95\% Cl 0.64 to 0.99; two studies, 3900 participants; Analysis 3.2), while a duration of six to 12 months had no impact on both moderate (RR 0.96, 95\% Cl 0.89 to 1.03; six studies; 10 comparisons); 7871 participants; Analysis 3.1) and severe stunting (RR 0.89, 95\% Cl 0.74 to 1.07; three studies; 7 comparisons); 2251 participants; Analysis 3.2).

There was no difference between duration of six to 12 months and more than 12 months on the prevalence of moderate wasting (Analysis 3.3).. All studies reporting on severe wasting provided intervention for six to 12 months only; there was no impact on severe wasting (RR 1.27, 95\% C: 0.66 to 2.46; three studies; 7 comparisons); 2329 participants; Analysis 3.4).

There was no difference in the impact of six to 12 months supplementation and more than 12 months supplementation for any of the other primary outcomes: moderate underweight (Analysis 3.5) and severe underweight (Analysis 3.6); anaemia (Analysis 3.7); and adverse effects (Analysis 3.8).

\section{Secondary outcomes}

A subgroup analysis by duration of intervention suggested that a duration of six to 12 months supplementation improved MUAC compared to a duration of less than six months and more than 12 months (SMD $0.18,95 \% \mathrm{Cl} 0.10$ to 0.26 ; three studies; 6 comparisons); 5114 participants; Analysis 3.9).

There was no difference in the impact of up to six months and 6 to 12 months supplementation for haemoglobin (Analysis 3.10).

All the studies reporting mortality provided intervention for six to 12 months suggesting no impact on mortality (RR 0.93 , 95\% $\mathrm{Cl} 0.63$ to 1.37; three studies (one of which contributed data to two comparisons; total number of comparisons = four), 3321 participants; Analysis 3.11).

\section{Explanatory secondary outcomes}

An intervention duration of up to six months (SMD $0.19,95 \% \mathrm{Cl} 0.01$ to 0.36; three studies; 1510 participants; Analysis 3.12) and more than 12 months improved HAZ (SMD: 0.14 ; 95\% Cl: 0.03 to 0.25 ; three studies; 5501 participants; Analysis 3.12), but had no impact on $\mathrm{HAZ}$ at six to 12 months.

An intervention duration of more than 12 months improved WAZ (SMD 0.13, 95\% Cl 0.03 to 0.24; three studies; 5541 participants; Analysis 3.13), but had no impact on WAZ at up to six months and six to 12 months.

An intervention duration of more than 12 months improved $\mathrm{WHZ}$ (SMD: 0.09 ; $95 \% \mathrm{Cl} 0.01$ to 0.17 ; three studies; 5503 participants; Analysis 3.14) while there was no impact of an intervention duration of up to six months and six to 12 months on WHZ (Analysis 3.14).
Age at follow-up (at 12 months; at 18 months; at 24 months; at 36 months)

\section{Primary outcomes}

A subgroup analysis by age at follow-up suggested a significant impact at 24 months of age on the prevalence of moderate stunting (RR 0.89, 95\% Cl 0.81 to 0.97, three studies, 5501 participants; Analysis 4.1) and severe stunting (RR $0.80,95 \% \mathrm{Cl} 0.64$ to 0.99 ; two studies, 3900 participants; Analysis 4.2), while there was no impact at the 12- and 18-month follow-up periods.

There was no difference between follow-up at 18 months of age and follow-up at 12 months for moderate wasting(Analysis 4.3) compared to follow-up at 12 months. There was no difference at 12 and 18 months of follow-up for severe wasting (Analysis 4.4).

There were no differences at 18 and 24 months of follow-up for moderate underweight (Analysis 4.5), or at 18 months follow-up for severe underweight (Analysis 4.6).

The impact on anaemia was also significant at follow-up at 18 months of age (RR $0.80,95 \% \mathrm{Cl} 0.71$, 0.91; three studies, 1156 participants; Analysis 4.7) compared to follow-up at 12 months of age.

There were no differences for adverse effects at 18 months followup (Analysis 4.8).

\section{Secondary outcomes}

A subgroup analysis by age at follow-up suggested significant impact of LNS plus complementary feeding on MUAC at 12month follow-up (SMD $0.15,95 \% \mathrm{Cl} 0.06$ to 0.24 ; one study; four comparisons); 1193 participants; Analysis 4.9), but not at the 18- or 24-month follow-up periods.

There was no difference at 12 months, 18 months and 36 months for haemoglobin (Analysis 4.10). There was no effect on mortality in any of the follow-up subgroups (Analysis 4.11).

\section{Explanatory secondary outcomes}

There was a significant impact on HAZ at 24 months follow-up (SMD: 0.14; 95\% Cl: 0.03 to 0.25; three studies; 5501 participants; Analysis 4.12) and at 36 months follow-up (SMD 0.27, 95\% Cl 0.10 to 0.44 ; one study, 1038 participants; Analysis 4.12), but no impact at 12 to 15 and 18 months follow-up.

There was a significant impact on WAZ at 24 months follow-up (SMD $0.13,95 \% \mathrm{Cl} 0.03$ to 0.23 ; three studies; 5541 participants; Analysis 4.13), but no impact at 12 to 15 months and 18 months follow-up.

There was a significant impact on WHZ at 24 months follow-up (SMD: $0.09 ; 95 \% \mathrm{Cl} 0.01$ to 0.17 ; three studies; 5503 participants; Analysis 4.14). There was no impact on WHZ at 12 to 15,18 or 36 months follow-up (Analysis 4.14).

\section{Comparison 2: LNS versus FBF}

Five studies with 12,135 participants compared LNS plus complementary feeding with FBF, including corn soy blend and UNIMIX (Bisimwa 2012; Christian 2015; Mangani 2015; Olney 2018; Phuka 2008). Of these, Olney 2018 did not contribute data towards meta-analysis, as it only reported outcomes according to age groups. 
We rated the quality of the evidence for all outcomes as low or moderate, as per GRADE criteria. We downgraded the quality of the evidence due to study limitations, imprecision and small sample size. See Summary of findings 2.

We did not conduct any subgroup analyses for this comparison due to insufficient data.

\section{Primary outcomes}

\section{Stunting: pooled study results}

Compared to FBF, LNS plus complementary feeding significantly reduced the prevalence of moderate stunting ( $\mathrm{RR} 0.89,95 \% \mathrm{Cl} 0.82$ to 0.97 ; three studies; 4 comparisons), 2828 participants; $1^{2}=0 \%$; $\mathrm{Tau}^{2}=0.00 ; \mathrm{Chi}^{2}=2.01 ;$ moderate-quality evidence; Analysis 5.1).

There was no difference between LNS plus complementary feeding and FBF for severe stunting (RR $0.41,95 \% \mathrm{Cl} 0.12$ to 1.42 ; two studies; 3 comparisons), 729 participants; $\mathrm{I}^{2}=57 \%$; $\mathrm{Tau}^{2}=0.68 ; \mathrm{Chi}^{2}$ = 4.68; low-quality evidence; Analysis 5.2).

\section{Wasting: pooled study results}

When compared to FBF, LNS plus complementary feeding significantly reduced the prevalence of moderate wasting (RR 0.79 , $95 \% \mathrm{Cl} 0.65$ to 0.97 ; two studies; 3 comparisons), 2290 participants; $\mathrm{I}^{2}=0 \% ; \mathrm{Tau}^{2}=0.00 ; \mathrm{Chi}^{2}=1.09 ;$ moderate-quality evidence; Analysis 5.3).

There was no difference between LNS plus complementary feeding and $\mathrm{FBF}$ for severe wasting (RR $0.64,95 \% \mathrm{Cl} 0.19$ to 2.18; two studies; 3 comparisons), 735 participants; $1^{2}=0 \%$; Tau $^{2}=0.00 ; \mathrm{Chi}^{2}$ $=0.32$; moderate-quality evidence; Analysis 5.4).

\section{Underweight}

\section{Pooled study results}

Compared to FBF, LNS plus complementary feeding significantly reduced the prevalence of moderate underweight (RR $0.81,95 \% \mathrm{Cl}$ 0.73 to 0.91 ; two studies; 3 comparisons), 2280 participants; $12=0 \%$; $\mathrm{Tau}^{2}=0.00 ; \mathrm{Chi}^{2}=0.00 ;$ moderate-quality evidence; Analysis 5.5).

\section{Single study results}

One study with two arms, Phuka 2008, reported no difference between LNS plus complementary feeding and FBF for severe underweight (RR $1.11,95 \% \mathrm{Cl} 0.46$ to $2.66 ; 173$ participants; lowquality evidence; Analysis 5.6).

\section{Psychomotor and neuro-developmental outcomes: single study results}

One study, Phuka 2008, reported comparable developmental outcomes in children given LNS plus complementary feeding and those given FBF and the outcome was rated to be of low quality.

None of the included studies reported data on other primary outcomes: anaemia and adverse effects.

\section{Secondary outcomes}

\section{MUAC: pooled study results}

There was no difference between LNS plus complementary feeding and FBF for MUAC (SMD $0.02,95 \% \mathrm{Cl}-0.08$ to 0.12 ; two studies; 3 comparisons), 1512 participants; $\mathrm{I}^{2}=0 \% ; \mathrm{Tau}^{2}=0.00 ; \mathrm{Chi}^{2}=0.07$; moderate-quality evidence; Analysis 5.7).

\section{Haemoglobin: single study results}

One study, Phuka 2008, which contributed data to two comparisons, found no difference between LNS plus complementary feeding and FBF for serum haemoglobin (MD $0.29,95 \% \mathrm{Cl}-6.00$ to $6.59 ; 182$ participants; low-quality outcome; Analysis 5.8).

None of the included studies reported data on our other secondary outcomes: morbidity and mortality.

\section{Explanatory secondary outcomes \\ HAZ: pooled study results}

There was no difference between LNS plus complementary feeding and FBF for HAZ (SMD $0.06,95 \% \mathrm{Cl} 0.00$ to 0.13 ; four studies; 5 comparisons), 4047 participants; $\mathrm{I}^{2}=2 \%$; $\mathrm{Tau}^{2}=0.00 ; \mathrm{Chi}^{2}=4.07$; moderate-quality evidence; Analysis 5.9).

\section{WAZ: pooled study results}

There was no difference between LNS plus complementary feeding and FBF for WAZ (MD $0.05,95 \% \mathrm{Cl}-0.04$ to 0.14 ; three studies; 4 comparisons), 1933 participants; $\left.\right|^{2}=5 \%$; Tau $^{2}=0.00 ;$ Chi $^{2}=3.17 ;$; moderate-quality evidence; Analysis 5.10).

\section{WHZ: pooled study results}

There was no difference between LNS plus complementary feeding and FBF for WHZ (SMD $0.07,95 \% \mathrm{Cl}-0.02$ to 0.16 ; three studies; 4 comparisons), 1933 participants; $\mathrm{I}^{2}=0 \%$; $\mathrm{Tau}^{2}=0.00 ; \mathrm{Chi}^{2}=2.17$; moderate-quality evidence; Analysis 5.11).

\section{Comparison 3: LNS versus nutritional counselling}

We did not find any study comparing LNS with nutritional counselling.

\section{Comparison 4: LNS versus micronutrient powders (MNP)}

Four studies with 9036 participants compared LNS plus complementary feeding with MNP (Adu-Afarwuah 2007; Dewey 2017; Matias 2017; Olney 2018). Of these, one study did not contribute data towards the meta-analysis, as it only reported outcomes according to age groups (Olney 2018).

We rated the quality of the evidence for all outcomes as low or moderate, as per GRADE criteria. We downgraded the quality of the evidence due to study limitations and small sample size. See Summary of findings 3.

We did not conduct any subgroup analyses for this comparison due to insufficient data.

\section{Primary outcomes}

\section{Stunting: pooled study results}

There was no difference between LNS plus complementary feeding and MNP on the prevalence of moderate stunting (RR $0.92,95 \% \mathrm{Cl}$ 0.82 to 1.02 ; three studies, 2365 participants; $1^{2}=0 \%$; $\mathrm{Tau}^{2}=0.00$; $\mathrm{Chi}^{2}=1.58$; moderate-quality evidence; Analysis 6.1). 


\section{Wasting: pooled study results}

There was no difference between LNS plus complementary feeding and MNP on the prevalence of moderate wasting (RR $0.97,95 \% \mathrm{Cl}$ 0.77 to 1.23 ; two studies, 2004 participants; $\mathrm{I}^{2}=0 \%$; $\mathrm{Tau}^{2}=0.00 ; \mathrm{Chi}^{2}$ = 0.58; moderate-quality evidence; Analysis 6.2).

\section{Underweight: pooled study results}

Compared to MNP, LNS plus complementary feeding significantly reduced the prevalence of moderate underweight (RR $0.88,95 \% \mathrm{Cl}$ 0.78 to 0.99 ; two studies, 2004 participants; $\mathrm{I}^{2}=0 \%$; $\mathrm{Tau}^{2}=0.00 ; \mathrm{Chi}^{2}$ $=0.31$; moderate-quality evidence; Analysis 6.3).

\section{Anaemia: pooled study results}

Two studies (557 participants) assessed this outcome (AduAfarwuah 2007; Matias 2017). We combined the data in a meta-analysis and found that, compared to MNP, LNS plus complementary feeding significantly reduced the prevalence of anaemia (RR 0.38, 95\% Cl 0.21 to $0.68 ; \mathrm{I}^{2}=53 \%$; $\mathrm{Tau}^{2}=0.10 ; \mathrm{Chi}^{2}=$ 2.14; low-quality evidence; Analysis 6.4).

We conducted a sensitivity analysis by removing Matias 2017 due to high risk of bias. We found that the effect on anaemia was no longer significant (RR $0.56,95 \% \mathrm{Cl} 0.27$ to 1.14; Analysis 6.5); however, the evidence is from one study only.

\section{Psychomotor development neuro-developmental outcomes: single} study results

One study with 422 participants (Matias 2017), reported on this outcome and found that, compared to MNP, the provision of LNS plus complementary feeding was associated with higher problemsolving task scores $(P=0.04)$ and increased odds of achieving this cognitive task (OR 1.6, $95 \% \mathrm{Cl} 1.0$ to 2.7). They observed no significant differences on receptive language or gross motor development.

\section{Any adverse effects}

None of the included studies reported data on adverse effects.

\section{Secondary outcomes}

\section{Haemoglobin: pooled study results}

Two studies (557 participants) reported data on haemoglobin (AduAfarwuah 2007; Matias 2017). We combined the data in a metaanalysis and found evidence of significantly higher haemoglobin in the LNS plus complementary feeding group compared to the MNP group (MD $5.13 \mathrm{~g} / \mathrm{L}, 95 \% \mathrm{Cl} 2.00$ to 8.26; $\mathrm{I}^{2}=25 \%$; $\mathrm{Tau}^{2}=1.28$; $\mathrm{Chi}^{2}$ $=1.33$; low-quality evidence; Analysis 6.6).

We conducted a sensitivity analysis by removing Matias 2017 due to high risk of bias. The effect on haemoglobin was no longer significant (MD 3.60 g/L, $95 \% \mathrm{Cl}-0.13$ to 7.33; Analysis 6.7); however, the evidence is from only one study.

None of the included studies reported data on our other secondary outcomes: MUAC; morbidity and mortality.

\section{Explanatory secondary outcomes}

\section{HAZ: pooled study results}

There was no difference between LNS plus complementary feeding and MNP for HAZ (SMD 0.10, 95\% Cl-0.08 to 0.27; three studies, 2362 participants; $I^{2}=63 \% ;$ Tau $^{2}=0.02 ;$ Chi $^{2}=5.47 ;$ moderate-quality evidence; Analysis 6.8).

We conducted a sensitivity analysis by removing Matias 2017 due to high risk of bias and found that the effect on HAZ became significant $\left(\mathrm{SMD} 0.16,95 \% \mathrm{Cl} 0.03\right.$ to $0.28 ; \mathrm{I}^{2}=19 \%$; $\mathrm{Tau}^{2}=0.00 ; \mathrm{Chi}^{2}=1.23$; Analysis 6.9).

\section{WAZ: pooled study results}

Compared to MNP, LNS plus complementary feeding improved WAZ (SMD 0.12, 95\% $\mathrm{Cl} 0.02$ to 0.21 ; three studies, 2362 participants; 12 = $10 \% ; \mathrm{Tau}^{2}=0.00 ; \mathrm{Chi}^{2}=2.23 ;$ moderate-quality evidence; Analysis 6.10).

We conducted a sensitivity analysis by removing Matias 2017 due to high risk of bias and found that the effect on WAZ remained significant (SMD 0.14, 95\% Cl 0.05 to $0.23 ; 1^{2}=0 \%$; $\mathrm{Tau}^{2}=0.00 ; \mathrm{Chi}^{2}$ =0.73; Analysis 6.11).

\section{WHZ: pooled study results}

There was no difference between LNS plus complementary feeding and MNP for WHZ (SMD 0.05, 95\% Cl -0.06 to 0.17 ; three studies, 2362 participants; $\mathrm{I}^{2}=29 \% ; \mathrm{Tau}^{2}=0.00 ; \mathrm{Chi}^{2}=2.80$; moderatequality evidence; Analysis 6.12).

We conducted a sensitivity analysis by removing Matias 2017 due to high risk of bias and found that the effect on WHZ became significant (SMD 0.10, $95 \% \mathrm{Cl} 0.01$ to $0.18 ; \mathrm{I}^{2}=0 \%$; $\mathrm{Tau}^{2}=0.00 ; \mathrm{Chi}^{2}$ =0.09; Analysis 6.13).

\section{DISCUSSION}

\section{Summary of main results}

This review summarises findings from 17 studies with 23,200 children. Each included study provided data on at least one of the primary outcomes.

Thirteen studies compared lipid-based nutrient supplements. (LNS) plus complementary feeding with no intervention. We included all 13 studies in meta-analyses We found evidence to suggest that LNS plus complementary feeding is effective in improving growth outcomes among children aged six to 23 months of age compared to complementary feeding alone. LNS plus complementary feeding significantly reduced the prevalence of moderate stunting by $7 \%$ ( $2 \%$ to $12 \%$ ), severe stunting by $15 \%$ ( $2 \%$ to $26 \%$ ), moderate wasting by $18 \%$ (9\% to $26 \%$ ), moderate underweight by $15 \%(9 \%$ to $20 \%)$ and anaemia by $21 \%(10 \%$ to $31 \%$ ). LNS plus complementary feeding also improved midupper arm circumference (MUAC), serum haemoglobin, height-forage $z$ score (HAZ), weight-for-age $z$ score (WAZ) and weight-forheight/length $z$ score $(\mathrm{WHZ})$. We found no impact of LNS plus complementary feeding on severe wasting, severe underweight and mortality. We rated the quality of the evidence as moderate to low for most outcomes, largely downgrading for lack of blinding, although this was expected due to the nature of the intervention. Adverse effects did not differ between the groups, suggesting that LNS plus complementary feeding is safe; however, studies did not report data on allergic reactions (including atopic dermatitis, urticaria, oedema, ophthalmic pruritus, allergic rhinitis, asthma, anaphylaxis). Although the data on psychomotor and neuro-developmental outcomes are scarce, a few studies 
found a positive impact of LNS plus complementary feeding on psychomotor outcomes (walking independently) compared to no intervention. We conducted subgroup analyses to explore the impact of energy content, duration of intervention and age at follow-up on the results. The subgroup analysis by energy content has limited clinical significance due to limited number of studies in each subgroup. The subgroup analysis by duration of intervention suggested that an intervention duration of more than 12 months reduced moderate stunting and severe stunting, while the subgroup analysis by age at follow-up suggested a significant impact on moderate stunting and severe stunting at 24 months follow-up and on moderate wasting at 18 months follow-up.

Four studies compared LNS plus complementary feeding with micronutrient powders (MNP). We combined data from three of these studies in meta-analyses. We found that LNS plus complementary feeding significantly reduced moderate underweight and anaemia and improved haemoglobin and WAZ. We found no difference between LNS plus complementary feeding and MNP for any of the other primary outcomes, including stunting, wasting and adverse effects. We rated the quality of the evidence for most outcomes as moderate to low, largely downgrading for lack of blinding. One study reported on psychomotor and neuro-developmental outcomes, suggesting that provision of LNS plus complementary feeding was associated with higher problemsolving task scores and increased odds of achieving this cognitive task compared to MNP; however, there was no difference in receptive language or gross motor development. other fortified blended foods (FBF), including corn soy blend and UNIMIX (UNICEF's supplementary feeding food). We were able to include four of these studies in meta-analyses. We found that LNS plus complementary feeding reduced moderate stunting, moderate wasting and moderate underweight. We found no difference between LNS plus complementary feeding and other FBF for severe stunting, severe wasting and severe underweight. We rated the quality of the evidence for most outcomes as moderate to low, largely downgrading for lack of blinding. One study reported comparable developmental outcomes in the LNS plus complementary feeding group compared to the FBF group.

We did not find any study comparing LNS alone with nutritional counselling alone to mothers and caregivers for appropriate feeding of infants and young children.

\section{Overall completeness and applicability of evidence}

This review summarises findings from 17 studies. All studies were published recently with the oldest one published in 2007. All studies were conducted in low- and middle-income countries (LMIC) in Sub-Saharan Africa and South Asia; none were conducted in emergency settings. Most studies compared LNS plus complementary feeding with no intervention, though some included MNP and other FBF as comparison groups. We did not find any study comparing LNS alone with nutritional counselling alone to mothers and caregivers for appropriate feeding of infants and young children. Studies assessing the impact of LNS plus complementary feeding on psychomotor and neurodevelopmental outcomes used various outcome definitions and measurement tools and hence could not be pooled together. Most studies used small quantity lipid-based nutrient supplements (SQ LNS), though a few studies also used medium quantity (MQ
Five studies compared LNS plus complementary feeding with

LNS) (MQ LNS). Four of the 17 included studies provided LNS plus complementary feeding to pregnant women and their infants (Adu-Afarwuah 2016; Ashorn 2015; Dewey 2017; Olney 2018). However, in one of these studies, there was also a group in which pregnant women did not receive LNS plus complementary feeding but the children did (Dewey 2017); we used the data from the group in which only children were provided with LNS plus complementary feeding compared to controls. We conducted a sensitivity analysis by removing studies in which pregnant women were also supplemented and found no difference in the estimates. We also conduced sensitivity analyses to assess the effects of removing trials at high risk of bias and the robustness of the results when using a fixed-effect model; there were no major changes in the estimates.

The findings of this review are generalisable to apparently healthy and non-hospitalised children in LMIC settings in Asia and Africa, although some children may be at risk of having highly prevalent diseases such as malaria, diarrhoea or even malnutrition. Use of LNS in the studies included in this review is limited for preventive purposes, and hence this review does not evaluate their effectiveness in treating any form of malnutrition.

\section{Quality of the evidence}

Overall, we considered most studies to be at low risk of bias for random sequence generation, allocation concealment, blinding of outcome assessment, incomplete outcome data and selective reporting, however, given inconsistency and imprecision of results, high risk of detection bias and lack of blinding the resulting judgements made using the Grade tool were that the quality of the evidence for those outcomes reported in the 'Summary of findings' tables was either low or moderate.

\section{Potential biases in the review process}

We were aware of the possibility of introducing bias at every stage of the reviewing process. In this review, we tried to minimise bias in a number of ways; two review authors independently assessed study eligibility for inclusion, carried out data extraction and assessed risk of bias and the quality of the evidence. While we attempted to be as inclusive as possible in our searches, the literature we identified was predominantly written in English. Although we attempted to assess reporting bias, this assessment relied largely on information available in the published studies and thus, reporting bias was not usually apparent.

\section{Agreements and disagreements with other studies or reviews}

To our knowledge, this is the first review assessing the impact of preventive LNS plus complementary feeding on children. The Kristjansson 2015 and Kristjansson 2016 review assessed the effectiveness of supplementary feeding interventions, alone or with a co-intervention, for improving the physical and psychosocial health of disadvantaged children aged three months to five years and suggested some positive impact of feeding on growth and moderate positive effects on psychomotor development. The review by Sguassero 2012 evaluated the effectiveness of community-based supplementary feeding for promoting the physical growth of children under five years of age in LMIC, highlighting the scarcity of evidence in reaching any firm conclusions. The review by Larson 2017 on nutrition interventions on mental development of children under two years of age in

Preventive lipid-based nutrient supplements given with complementary foods to infants and young children 6 to 23 months of age for 27 health, nutrition, and developmental outcomes (Review)

Copyright (c) 2019 The Cochrane Collaboration. Published by John Wiley \& Sons, Ltd. 
LMIC suggested small effects of nutrition interventions on mental development.

\section{AUTHORS' CONCLUSIONS}

\section{Implications for practice}

The findings of this review suggest improved growth outcomes when lipid-based nutrient supplements (LNS) are provided with complementary feeding to apparently healthy, non-hospitalised children aged six to 23 months in low- and middle-income countries (LMIC) settings. LNS plus complementary feeding reduced the prevalence of moderate and severe stunting, moderate wasting and moderate underweight, and improved mid-upper arm circumference (MUAC), serum haemoglobin, height-for-age $z$ score (HAZ), weight-for-age z score (WAZ) and weight-for-height/ length $\mathrm{z}$ score (WHZ). LNS plus complementary feeding was more effective when the duration of the intervention was longer than 12 months. Subgroup analyses according to energy content were not clinically significant due to the limited number of studies in each subgroup. Data comparing LNS plus complementary feeding with other products are limited; however, when compared to micronutrient powders (MNP), LNS plus complementary feeding significantly reduced moderate underweight and improved WAZ. When compared to other fortified blended foods (FBF), including corn soy blend and (UNICEF's supplementary feeding food (UNIMIX), LNS plus complementary feeding reduced moderate stunting, moderate wasting and moderate underweight. These findings are applicable to other Asian and African countries with similar prevalences for undernutrition, food insecurity, and where short-term food supplementation is required for the prevention of stunting and wasting.

Findings from this review suggest that LNS plus complementary feeding may prevent undernutrition and improve growth in children aged six to 23 months in LMIC; although we did not find any study comparing LNS alone with nutritional counselling alone to mothers and caregivers for appropriate feeding of infants and young children. Measurement of dietary intake at baseline and during the intervention could provide information on average energy intake, macronutrient and micronutrient composition of baseline diets or energy received from LNS plus complementary feeding compared to household foods during the intervention, to inform whether the supplement displaced usual intake.

\section{Implications for research}

The results of our review provide a number of implications for future research. First, there are no existing data on the impact of preventive LNS plus complementary feeding in emergency settings, yet LNS are already being used for children in emergency settings. Second, there is a scarcity of data on the impact of LNS plus complementary feeding on psychomotor and neurodevelopmental outcomes. Future studies should measure all relevant child development domains in a standardised manner so that they can be pooled in a meta-analysis. Further research is needed to evaluate the relative effectiveness of LNS provision in addition to complementary feeding compared to nutrition education alone and to other products. There is a need to evaluate the preventive impact of LNS plus complementary feeding for longer durations and at longer follow-up periods to capture the long-term impact of LNS plus complementary feeding interventions in infants and young children.

\section{ACKNOWLEDGEMENTS}

We are grateful to the Cochrane Developmental, Psychosocial and Learning Problems editorial team for their support in the preparation of this review. We are also thankful to the four peers (a content editor, a statistical editor, and two reviewers who are external to the editorial team) who commented on the review.

The review was partially developed during the World Health Organization/Cochrane/Cornell University Summer Institute for Systematic Reviews in Nutrition Global Policy Making, hosted at the Division of Nutritional Sciences, Cornell University, Ithaca, USA, from 25 July to 5 August 2016.

We would like to acknowledge Nida Ashraf for assisting with the preliminary data extraction and analysis.

We acknowledge Kathryn Dewey (KD) who reviewed the draft meticulously and provided critical input, especially on the technical aspects of the intervention and methodological aspects of the studies of which she was Principal Investigator. KD had no role in finalising the findings and conclusions of the review. 


\section{R E F E R E N C E S}

\section{References to studies included in this review}

\section{Adu-Afarwuah 2007 \{published data only\}}

Adu-Afarwuah S, Lartey A, Brown KH, Zlotkin S, Briend A, Dewey KG. Home fortification of complementary foods with micronutrient supplements is well accepted and has positive effects on infant iron status in Ghana. American Journal of Clinical Nutrition 2008;87(4):929-38. [DOI: 10.1093/ ajcn/87.4.929; PUBMED: 18400716]

* Adu-Afarwuah S, Lartey A, Brown KH, Zlotkin S, Briend A, Dewey KG. Randomized comparison of 3 types of micronutrient supplements for home fortification of complementary foods in Ghana: effects on growth and motor development. American Journal of Clinical Nutrition 2007;86(2):412-20. [DOI: 10.1093/ ajcn/86.2.412; PUBMED: 17684213]

\section{Adu-Afarwuah 2016 \{published data only\}}

Adams KP, Lybbert TJ, Vosti SA, Ayifah E, Arimond M, AduAfarwuah S, et al. Unintended effects of a targeted maternal and child nutrition intervention on household expenditures, labor income, and the nutritional status of non-targeted siblings in Ghana. World Development 2018;107:138-50. [DOI: 10.1016/ j.worlddev.2018.02.025; PMC5917415; PUBMED: 29970953]

Adams KP, Okronipa H, Adu-Afarwuah S, Arimond M, Kumordzie S, Oaks BM, et al. Ghanaian parents' perceptions of pre and postnatal nutrient supplements and their effects. Maternal \& Child Nutrition 2018;14(4):e12608. [DOI: 10.1111/ mcn.12608; PUBMED: 29656569]

Adu-Afarwuah S, Lartey A, Okronipa H, Ashorn P, Ashorn U, Zeilani M, et al. Maternal supplementation with small-quantity lipid-based nutrient supplements compared with multiple micronutrients, but not with iron and folic acid, reduces the prevalence of low gestational weight gain in semi-urban Ghana: a randomized controlled trial. Journal of Nutrition 2017;147(4):697-705. [DOI: 10.3945/jn.116.242909; PMC5368579; PUBMED: 28275100]

* Adu-Afarwuah S, Lartey A, Okronipa H, Ashorn P, Peerson JM, Arimond $\mathrm{M}$, et al. Small-quantity, lipid-based nutrient supplements provided to women during pregnancy and $6 \mathrm{mo}$ postpartum and to their infants from 6 mo of age increase the mean attained length of 18 -mo-old children in semiurban Ghana: a randomized controlled trial. American Journal of Clinical Nutrition 2016;104(3):797-808. [DOI: 10.3945/ ajcn.116.134692; PMC4997301; PUBMED: 27534634]

Adu-Afarwuah S, Young RT, Lartey A, Okronipa H, Ashorn P, Ashorn U, et al. Supplementation during pregnancy with small-quantity lipid-based nutrient supplements or multiple micronutrients, compared with iron and folic acid, increases women's urinary iodine concentration in semiurban Ghana: a randomized controlled trial. Maternal \& Child Nutrition 2018;14(2):e12570. [DOI: 10.1111/mcn.12570; PMC5900724; PUBMED: 29210520]

Okronipa H, Adu-Afarwuah S, Lartey A, Ashorn P, Vosti SA, Young RR, et al. Maternal supplementation with small-quantity lipid-based nutrient supplements during pregnancy and lactation does not reduce depressive symptoms at 6 months postpartum in Ghanaian women: a randomized controlled trial. Archives of Women's Mental Health 2018;21(1):55-63. [DOI: 10.1007/s00737-017-0752-7; PMC5762799; PUBMED: 28698916]

Prado EL, Adu-Afarwuah S, Lartey A, Ocansey M, Ashorn P, Vosti SA, et al. Effects of pre- and post-natal lipid-based nutrient supplements on infant development in a randomized trial in Ghana. Early Human Development 2016;99:43-51. [DOI: 10.1016/ j.earlhumdev.2016.05.011; PUBMED: 27391572]

\section{Ashorn 2015 \{published data only\}}

* Ashorn P, Alho L, Ashorn U, Cheung YB, Dewey KG, Gondwe A, et al. Supplementation of maternal diets during pregnancy and for 6 months postpartum and infant diets thereafter with smallquantity lipid-based nutrient supplements does not promote child growth by 18 months of age in rural Malawi: a randomized controlled trial. Journal of Nutrition 2015;145(6):1345-53. [DOI: 10.3945/jn.114.207225; PUBMED: 25926413]

Ashorn P, Alho L, Ashorn U, Cheung YB, Dewey KG, Harjunmaa $U$, et al. The impact of lipid-based nutrient supplement provision to pregnant women on newborn size in rural Malawi: a randomized controlled trial. American Journal of Clinical Nutrition 2015;101(2):387-97. [DOI: 10.3945/ ajcn.114.088617; PUBMED: 25646337]

Barua P, Chandrasiri UP, Beeson JG, Dewey KG, Maleta K, Ashorn $P$, et al. Effect of nutrient supplementation on the acquisition of humoral immunity to Plasmodium falciparum in young Malawian children. Malaria Journal 2018;17(1):74. [DOI: 10.1186/s12936-018-2224-6; PMC5804088; PUBMED: 29415730]

Jorgensen JM, Arnold C, Ashorn P, Ashorn U, Chaima D, Cheung YB, et al. Lipid-based nutrient supplements during pregnancy and lactation did not affect human milk oligosaccharides and bioactive proteins in a randomized trial. Journal of Nutrition 2017;147(10):1867-74. [DOI: 10.3945/ jn.117.252981; PMC5610548; PUBMED: 28794206]

Prado EL, Ashorn U, Phuka J, Maleta K, Sadalaki J, Oaks BM, et al. Associations of maternal nutrition during pregnancy and post-partum with maternal cognition and caregiving. Maternal \& Child Nutrition 2018;14(2):e12546. [DOI: 10.1111/mcn.12546; PMC5901033; PUBMED: 29098783]

Prado EL, Maleta K, Ashorn P, Ashorn U, Vosti SA, Sadalaki J, et al. Effects of maternal and child lipid-based nutrient supplements on infant development: a randomized trial in Malawi. American Journal of Clinical Nutrition 2016;103(3):784-93. [DOI: 10.3945/ajcn.115.114579; PUBMED: 26843155]

\section{Bisimwa 2012 \{published data only\}}

Bisimwa G, Owino VO, Bahwere P, Dramaix M, Donnen P, Dibari $F$, et al. Randomized controlled trial of the effectiveness of a soybean-maize-sorghum-based ready-to-use complementary food paste on infant growth in South Kivu, Democratic Republic of Congo. American Journal of Clinical Nutrition 2012;95(5):1157-64. [DOI: 10.3945/ajcn.111.028704; PUBMED: 22492382] 


\section{Christian 2015 \{published data only\}}

Christian P, Shaikh S, Shamim AA, Mehra S, Wu L, Mitra M, et al. Effect of fortified complementary food supplementation on child growth in rural Bangladesh: a cluster-randomized trial. International Journal of Epidemiology 2015;44(6):1862-76. [DOI: 10.1093/ije/dyv155; PMC4689999; PUBMED: 26275453]

\section{Dewey 2017 \{published data only\}}

* Dewey KG, Mridha MK, Matias SL, Arnold CD, Cummins JR, Khan MS, et al. Lipid-based nutrient supplementation in the first $1000 \mathrm{~d}$ improves child growth in Bangladesh: a clusterrandomized effectiveness trial. American Journal of Clinical Nutrition 2017;105(4):944-57. [DOI: 10.3945/ajcn.116.147942; PUBMED: 28275125]

Matias SL, Mridha MK, Tofail F, Arnold CD, Khan MS, Siddiqui Z, et al. Home fortification during the first $1000 \mathrm{~d}$ improves child development in Bangladesh: a cluster-randomized effectiveness trial. American Journal of Clinical Nutrition 2017;105(4):958-69. [DOI: 10.3945/ajcn.116.150318; PUBMED: 28275128]

Matias SL, Mridha MK, Young RT, Khan MS, Siddiqui Z, Ullah MB, et al. Prenatal and postnatal supplementation with lipid-based nutrient supplements reduces anemia and iron deficiency in 18-month-old Bangladeshi children: a cluster-randomized effectiveness trial. Journal of Nutrition 2018;148(7):1167-76. [DOI: 10.1093/jn/nxy078; PUBMED: 29901736]

\section{Hess 2015 \{published data only\}}

Abbeddou S, Jimenez EY, Somé JW, Ouédraogo JB, Brown KH, Hess SY. Small-quantity lipid-based nutrient supplements containing different amounts of zinc along with diarrhea and malaria treatment increase iron and vitamin A status and reduce anemia prevalence, but do not affect zinc status in young Burkinabe children: a cluster-randomized trial. BMC pediatrics 2017;17(1):46. [DOI: 10.1186/s12887-016-0765-9; PMC5288861; PUBMED: 28152989]

Hess SY, Abbeddou S, Jimenez EY, Ouédraogo JB, Brown KH. lodine status of young Burkinabe children receiving smallquantity lipid-based nutrient supplements and iodised salt: a cluster-randomised trial. British Journal of Nutrition 2015;114(11):1829-37. [DOI: 10.1017/S0007114515003554; PUBMED: 26411504$]$

* Hess SY, Abbeddou S, Jimenez EY, Somé JW, Vosti SA, Ouédraogo ZP, et al. Small-quantity lipid-based nutrient supplements, regardless of their zinc content, increase growth and reduce the prevalence of stunting and wasting in young Burkinabe children: a cluster-randomized trial. PLOS One 2015;10(3):e0122242. [DOI: 10.1371/journal.pone.0122242; PMC4376671; PUBMED: 25816354]

Prado EL, Abbeddou S, Jimenez EY, Somé JW, Ouédraogo ZP, Vosti SA, et al. Lipid-based nutrient supplements plus malaria and diarrhea treatment increase infant development scores in a cluster-randomized trial in Burkina Faso. Journal of Nutrition 2016;146(4):814-22. [DOI: 10.3945/jn.115.225524; PUBMED: 26962193]

Somé JW, Abbeddou S, Jimenez EY, Hess SY, Ouédraogo ZP, Guissou RM, et al. Effect of zinc added to a daily small-quantity lipid-based nutrient supplement on diarrhoea, malaria, fever and respiratory infections in young children in rural Burkina Faso: a cluster-randomised trial. BMJ Open 2015;5(9):e007828. [DOI: 10.1136/bmjopen-2015-007828; PMC4567679; PUBMED: 26362661]

\section{Huybregts 2012 \{published data only\}}

Huybregts L, Houngbé F, Salpéteur C, Brown R, Roberfroid D, Ait-Aissa $\mathrm{M}$, et al. The effect of adding ready-to-use supplementary food to a general food distribution on child nutritional status and morbidity: a cluster-randomized controlled trial. PLOS Medicine 2012;9(9):e1001313. [DOI: 10.1371/journal.pmed.1001313; PMC3445445; PUBMED: 23028263]

\section{lannotti 2014 \{published data only\}}

* Iannotti LL, Dulience SJ, Green J, Joseph S, François J, Anténor ML, et al. Linear growth increased in young children in an urban slum of Haiti: a randomized controlled trial of a lipid-based nutrient supplement. American Journal of Clinical Nutrition 2014;99(1):198-208. [DOI: 10.3945/ajcn.113.063883; PMC3862455; PUBMED: 24225356]

Lesorogol C, Jean-Louis S, Green J, Iannotti L. Preventative lipid-based nutrient supplements (LNS) and young child feeding practices: findings from qualitative research in Haiti. Maternal \& Child Nutrition 2015;11(Suppl 4):62-76. [DOI: 10.1111/ mcn.12122; PUBMED: 24784976]

\section{Kumwenda 2014 \{published data only\}}

Bendabenda J, Alho L, Ashorn U, Cheung YB, Dewey KG, Vosti SA, et al. The effect of providing lipid-based nutrient supplements on morbidity in rural Malawian infants and young children: a randomized controlled trial. Public Health Nutrition 2016;19(10):1893-903. [DOI: 10.1017/S1368980016000331; PUBMED: 26956611]

Hemsworth J, Kumwenda C, Arimond M, Maleta K, Phuka J, Rehman AM, et al. Lipid-based nutrient supplements increase energy and macronutrient intakes from complementary food among Malawian infants. Journal of Nutrition 2016;146(2):326-34. [DOI: 10.3945/jn.115.215327; PUBMED: 26740684]

* Kumwenda C, Dewey KG, Hemsworth J, Ashorn P, Maleta K, Haskell MJ. Lipid-based nutrient supplements do not decrease breast milk intake of Malawian infants. American Journal of Clinical Nutrition 2014;99(3):617-23. [DOI: 10.3945/ ajcn.113.076588; PUBMED: 24368436]

Maleta KM, Phuka J, Alho L, Cheung YB, Dewey KG, Ashorn U, et al. Provision of 10-40 g/d lipid-based nutrient supplements from 6 to 18 months of age does not prevent linear growth faltering in Malawi. Journal of Nutrition 2015;145(8):1909-15. [DOI: 10.3945/jn.114.208181; PUBMED: 26063066]

Prado EL, Phuka J, Maleta K, Ashorn P, Ashorn U, Vosti SA, et al. Provision of lipid-based nutrient supplements from age 6 to 18 months does not affect infant development scores in a randomized trial in Malawi. Maternal and Child Health Journal 2016;20(10):2199-208. [DOI: 10.1007/s10995-016-2061-6; PUBMED: 27395385]

Preventive lipid-based nutrient supplements given with complementary foods to infants and young children 6 to 23 months of age for health, nutrition, and developmental outcomes (Review)

Copyright $\odot 2019$ The Cochrane Collaboration. Published by John Wiley \& Sons, Ltd. 
Pulakka A, Ashorn U, Cheung YB, Dewey KG, Maleta K, Vosti SA, et al. Effect of 12-month intervention with lipid-based nutrient supplements on physical activity of 18-month-old Malawian children: a randomised, controlled trial. European Journal of Clinical Nutrition 2015;69(2):173-8. [DOI: 10.1038/ejcn.2014.138; PUBMED: 25028082]

\section{Luby 2018 \{published data only\}}

Jannat K, Luby SP, Unicomb L, Rahman M, Winch PJ, Parvez SM, et al. Complementary feeding practices among rural Bangladeshi mothers: results from WASH Benefits study. Maternal \& Child Nutrition 2019;15(1):e12654. [DOI: 10.1111/ mcn.12654; PUBMED: 30101576]

* Luby SP, Rahman M, Arnold BF, Unicomb L, Ashraf S, Winch PJ, et al. Effects of water quality, sanitation, handwashing, and nutritional interventions on diarrhoea and child growth in rural Bangladesh: a cluster randomised controlled trial. Lancet Global Health 2018;6(3):e302-15. [DOI: 10.1016/ S2214-109X(17)30490-4]

Parvez SM, Azad R, Rahman M, Unicomb L, Ram PK, Naser AM, et al. Achieving optimal technology and behavioral uptake of single and combined interventions of water, sanitation hygiene and nutrition, in an efficacy trial (WASH benefits) in rural Bangladesh. Trials 2018;19(1):358. [DOI: 10.1186/ s13063-018-2710-8; PMC6034207; PUBMED: 29976251]

Rahman M, Ashraf S, Unicomb L, Mainuddin AK, Parvez SM, Begum F, et al. WASH Benefits Bangladesh trial: system for monitoring coverage and quality in an efficacy trial. Trials 2018;19:360. [DOI: 10.1186/s13063-018-2708-2]

Tofail F, Fernald LC, Das KK, Rahman M, Ahmed T, Jannat KK, et al. Effect of water quality, sanitation, hand washing, and nutritional interventions on child development in rural Bangladesh (WASH Benefits Bangladesh): a clusterrandomised controlled trial. Lancet Child \& Adolescent Health 2018;2(4):255-68. [DOI: 10.1016/S2352-4642(18)30031-2; PMC5859216; PUBMED: 29616235]

\section{Mangani 2015 \{published data only\}}

Aakko J, Grześkowiak Ł, Asukas T, Päivänsäde E, Lehto KM, Fan YM, et al. Lipid-based nutrient supplements do not affect gut bifidobacterium microbiota in Malawian infants: a randomized trial. Journal of Pediatric Gastroenterology \& Nutrition 2017;64(4):610-5. [DOI: 10.1097/ MPG.0000000000001333; PUBMED: 27403608]

Mangani C, Ashorn P, Maleta K, Phuka J, Thakwalakwa C, Dewey K, et al. Lipid-based nutrient supplements do not affect the risk of malaria or respiratory morbidity in 6-to 18-monthold Malawian children in a randomized controlled trial. Journal of Nutrition 2014;144(11):1835-42. [DOI: 10.3945/jn.114.196139; PUBMED: 25332483]

Mangani C, Cheung YB, Maleta K, Phuka J, Thakwalakwa C, Dewey K, et al. Providing lipid-based nutrient supplements does not affect developmental milestones among Malawian children. Acta Paediatrica 2014;103(1):e17-26. [DOI: 10.1111/apa.12443; PUBMED: 24118040]
* Mangani C, Maleta K, Phuka J, Cheung YB, Thakwalakwa C, Dewey $\mathrm{K}$, et al. Effect of complementary feeding with lipidbased nutrient supplements and corn-soy blend on the incidence of stunting and linear growth among 6-to 18-monthold infants and children in rural Malawi. Maternal \& Child Nutrition 2015;11(Suppl 4):132-43. [DOI: 10.1111/mcn.12068; PUBMED: 23795976]

\section{Matias 2017 \{published data only\}}

Matias SL, Vargas-Vásquez A, Pérez RB, Valdivia LA, Vivanco OA, Martín AR, et al. Effects of lipid-based nutrient supplements $\checkmark$ micronutrient powders on nutritional and developmental outcomes among Peruvian infants. Public Health Nutrition 2017;20(16):2998-3007. [DOI: 10.1017/S1368980017001811; PUBMED: 28789712]

\section{Null 2018 \{published data only\}}

Byrd K, Dentz HN, Williams A, Kiprotich M, Pickering AJ, Omondi R, et al. A behaviour change intervention with lipidbased nutrient supplements had little impact on young child feeding indicators in rural Kenya. Maternal \& Child Nutrition 2019;15(1):e12660. [DOI: 10.1111/mcn.12660; PUBMED: 30207423]

* Null C, Stewart CP, Pickering AJ, Dentz HN, Arnold BF, Arnold CD, et al. Effects of water quality, sanitation, handwashing, and nutritional interventions on diarrhoea and child growth in rural Kenya: a cluster-randomised controlled trial. Lancet Global Health 2018;6(3):e316-29. [DOI: 10.1016/ S2214-109X(18)30005-6; PMC5809717; PUBMED: 29396219]

Stewart CP, Kariger P, Fernald L, Pickering AJ, Arnold CD, Arnold BF, et al. Effects of water quality, sanitation, handwashing, and nutritional interventions on child development in rural Kenya (WASH Benefits Kenya): a clusterrandomised controlled trial. Lancet Child \& Adolescent Health 2018;2(4):269-80.

\section{Olney 2018 \{published data only\}}

Olney DK, Leroy J, Bliznashka L, Ruel MT. PROCOMIDA, a food-assisted maternal and child health and nutrition program, reduces child stunting in Guatemala: a clusterrandomized controlled intervention trial. Journal of Nutrition 2018;148(9):1493-505. [DOI: 10.1093/jn/nxy138; PMC6118165; PUBMED: 30184223$]$

\section{Phuka 2008 \{published data only\}}

Phuka J, Ashorn U, Ashorn P, Zeilani M, Cheung YB, Dewey KG, et al. Acceptability of three novel lipid-based nutrient supplements among Malawian infants and their caregivers. Maternal \& Child Nutrition 2011;7(4):368-77. [DOI: 10.1111/ j.1740-8709.2011.00297.x; PUBMED: 21518250]

Phuka JC, Gladstone M, Maleta K, Thakwalakwa C, Cheung YB, Briend A, et al. Developmental outcomes among 18-monthold Malawians after a year of complementary feeding with lipid-based nutrient supplements or corn-soy flour. Maternal \& Child Nutrition 2012;8(2):239-48. [DOI: 10.1111/ j.1740-8709.2011.00294.x; PUBMED: 21342456]

* Phuka JC, Maleta K, Thakwalakwa C, Cheung YB, Briend A, Manary MJ, et al. Complementary feeding with fortified 
spread and incidence of severe stunting in 6-to 18-monthold rural Malawians. Archives of Pediatrics \& Adolescent Medicine 2008;162(7):619-26. [DOI: 10.1001/archpedi.162.7.619; PMC3721756; PUBMED: 18606932]

Phuka JC, Maleta K, Thakwalakwa C, Cheung YB, Briend A, Manary MJ, et al. Postintervention growth of Malawian children who received 12-mo dietary complementation with a lipidbased nutrient supplement or maize-soy flour. American Journal of Clinical Nutrition 2009;89(1):382-90. [DOI: 10.3945/ ajcn.2008.26483; PUBMED: 19056572]

\section{Siega-Riz 2014 \{published data only\}}

Flax VL, Siega-Riz AM, Reinhart GA, Bentley ME. Provision of lipid-based nutrient supplements to Honduran children increases their dietary macro- and micronutrient intake without displacing other foods. Maternal \& Child Nutrition 2015;11(Suppl 4):203-13. [DOI: 10.1111/mcn.12182; PUBMED: 25819697]

* Siega-Riz AM, Estrada Del Campo Y, Kinlaw A, Reinhart GA, Allen LH, Shahab-Ferdows S, et al. Effect of supplementation with a lipid-based nutrient supplement on the micronutrient status of children aged 6-18 months living in the rural region of Intibucá, Honduras. Paediatric and Perinatal Epidemiology 2014;28(3):245-54. [DOI: 10.1111/ppe.12117; PUBMED: 24628577]

\section{References to studies excluded from this review}

\section{Ackatia-Armah 2015 \{published data only\}}

Ackatia-Armah RS, McDonald CM, Doumbia S, Erhardt JG, Hamer DH, Brown KH. Malian children with moderate acute malnutrition who are treated with lipid-based dietary supplements have greater weight gains and recovery rates than those treated with locally produced cereal-legume products: a community-based, cluster-randomized trial. American Journal of Clinical Nutrition 2015;101(3):632-45. [DOI: 10.3945/ ajcn.113.069807; PUBMED: 25733649]

\section{Adams 2017 \{published data only\}}

Adams KP, Ayifah E, Phiri TE, Mridha MK, Adu-Afarwuah S, Arimond $\mathrm{M}$, et al. Maternal and child supplementation with lipid-based nutrient supplements, but not child supplementation alone, decreases self-reported household food insecurity in some settings. Journal of Nutrition 2017;147(12):2309-18. [DOI: 10.3945/jn.117.257386; PMC5697970; PUBMED: 28978680]

\section{Adams 2018 \{published data only\}}

Adams KP, Vosti SA, Ayifah E, Phiri TE, Adu-Afarwuah S, Maleta $\mathrm{K}$, et al. Willingness to pay for small-quantity lipidbased nutrient supplements for women and children: evidence from Ghana and Malawi. Maternal \& Child Nutrition 2018;14(2):e12518. [DOI: 10.1111/mcn.12518; PMC6088232; PUBMED: 28960913]

\section{Ahmed 2014 \{published data only\}}

Ahmed T, Choudhury N, Hossain MI, Tangsuphoom N, Islam MM, de Pee S, et al. Development and acceptability testing of readyto-use supplementary food made from locally available food ingredients in Bangladesh. BMC Pediatrics 2014;14:164. [DOI: 10.1186/1471-2431-14-164; PMC4098698; PUBMED: 24972632]

Arimond 2017 \{published data only\}

Arimond M, Abbeddou S, Kumwenda C, Okronipa $\mathrm{H}$, Hemsworth J, Jimenez EY, et al. Impact of small quantity lipid-based nutrient supplements on infant and young child feeding practices at 18 months of age: results from four randomized controlled trials in Africa. Maternal \& Child Nutrition 2017;13(3):e12377. [DOI: 10.1111/mcn.12377; PMC5516197; PUBMED: 27910260]

\section{Cercamondi 2013 \{published data only\}}

Cercamondi Cl, Egli IM, Mitchikpe E, Tossou F, Hessou J, Zeder C, et al. Iron bioavailability from a lipid-based complementary food fortificant mixed with millet porridge can be optimized by adding phytase and ascorbic acid but not by using a mixture of ferrous sulfate and sodium iron EDTA. Journal of Nutrition 2013;143(8):1233-9. [DOI: 10.3945/jn.113.175075; PUBMED: 23761652]

\section{Defourney 2009 \{published data only\}}

Defourny I, Minetti A, Harczi G, Doyon S, Shepherd S, Tectonidis M, et al. A large-scale distribution of milk-based fortified spreads: evidence for a new approach in regions with high burden of acute malnutrition. PLOS One 2009;4(5):e5455. [DOI: 10.1371/journal.pone.0005455; PMC2673585; PUBMED: 19421316]

\section{Flax 2010 \{published data only\}}

Flax VL, Phuka J, Cheung YB, Ashorn U, Maleta K, Ashorn P. Feeding patterns and behaviors during home supplementation of underweight Malawian children with lipid-based nutrient supplements or corn-soy blend. Appetite 2010;54(3):504-11. [DOI: 10.1016/j.appet.2010.02.003; PUBMED: 20153389]

\section{Flax 2013 \{published data only\}}

Flax VL, Bentley ME, Chasela CS, Kayira D, Hudgens MG, Kacheche KZ, et al. Lipid-based nutrient supplements are feasible as a breastmilk replacement for HIV-exposed infants from 24 to 48 weeks of age. Journal of Nutrition 2013;143(5):701-7. [DOI: 10.3945/jn.112.168245; PMC3738238; PUBMED: 23468553]

\section{Heidkamp 2012 \{published data only\}}

Heidkamp RA, Stoltzfus RJ, Fitzgerald DW, Pape JW. Growth in late infancy among HIV-exposed children in urban Haiti is associated with participation in a clinic-based infant feeding support intervention. Journal of Nutrition 2012;142(4):774-80. [DOI: 10.3945/jn.111.155275; PMC3301993; PUBMED: 22378328]

\section{Isanaka 2009 \{published data only\}}

* Isanaka S, Nombela N, Djibo A, Poupard M, Van Beckhoven D, Gaboulaud V, et al. Effect of preventive supplementation with ready-to-use therapeutic food on the nutritional status, mortality, and morbidity of children aged 6 to 60 months in Niger: a cluster randomized trial. JAMA 2009;301(3):277-85. [DOI: 10.1001/jama.2008.1018; PMC3144630 ; PUBMED: 19155454] 
Isanaka S, Roederer T, Djibo A, Luquero FJ, Nombela N, Guerin PJ, et al. Reducing wasting in young children with preventive supplementation: a cohort study in Niger. Pediatrics 2010;126(2):e442-50. [DOI: 10.1542/peds.2009-2814; PMC3144628; PUBMED: 20660552]

\section{luel-Brockdorf 2015 \{published data only\}}

luel-Brockdorf AS, Dræbel TA, Fabiansen C, Cichon B, Christensen VB, Yameogo C, et al. Acceptability of new formulations of corn-soy blends and lipid-based nutrient supplements in Province du Passoré, Burkina Faso. Appetite 2015;91(1):278-86. [DOI: 10.1016/j.appet.2015.04.058; PUBMED: 25913687]

\section{Kuusipalo 2006 \{published data only\}}

Kuusipalo H, Maleta K, Briend A, Manary M, Ashorn P. Growth and change in blood haemoglobin concentration among underweight Malawian infants receiving fortified spreads for 12 weeks: a preliminary trial. Journal of Pediatric Gastroenterology and Nutrition 2006;43(4):525-32. [DOI: 10.1097/01.mpg.0000235981.26700.d3; PUBMED: 17033530]

\section{LaGrone 2012 \{published data only\}}

LaGrone LN, Trehan I, Meuli GJ, Wang RJ, Thakwalakwa C, Maleta K, et al. A novel fortified blended flour, corn-soy blend "plus-plus," is not inferior to lipid-based ready-to-use supplementary foods for the treatment of moderate acute malnutrition in Malawian children. American Journal of Clinical Nutrition 2012;95(1):212-9. [DOI: 10.3945/ajcn.111.022525; PMC3238461; PUBMED: 22170366]

\section{Langendorf 2014 \{published data only\}}

* Langendorf C, Roederer T, de Pee S, Brown D, Doyon S, Mamaty AA, et al. Preventing acute malnutrition among young children in crises: a prospective intervention study in Niger. PLOS Medicine 2014;11(9):e1001714. [DOI: 10.1371/ journal.pmed.1001714; PMC4152259; PUBMED: 25180584]

Prudhon C, Langendorf C, Roederer T, Doyon S, Mamaty AA, Woi-Messe L, et al. Effect of ready-to-use foods for preventing child undernutrition in Niger: analysis of a prospective intervention study over 15 months of follow-up. Maternal \& Child Nutrition 2017;13(1):e12236. [DOI: 10.1111/mcn.12236; PUBMED: 26775560]

Sayyad-Neerkorn J, Langendorf C, Roederer T, Doyon S, Mamaty AA, Woi-Messe L, et al. Preventive effects of long-term supplementation with 2 nutritious food supplements in young children in Niger. Journal of Nutrition 2015;145(11):2596-603. [DOI: 10.3945/jn.115.213157; PUBMED: 26423742]

\section{Maleta 2004 \{published data only\}}

Maleta K, Kuittinen J, Duggan MB, Briend A, Manary M, Wales J, et al. Supplementary feeding of underweight, stunted Malawian children with a ready-to-use food. Journal of Pediatric Gastroenterology and Nutrition 2004;38(2):152-8. [PUBMED: 14734876]

\section{Maryam 2015 \{published data only\}}

Maryam A, Hadju V, As'ad S, Bahar B. Factors related to the status of vitamin $A$ and zink baduta aged 6-23 months in the district of East Central South, Province of Nusa
Tenggara Timur. International Journal of Sciences: Basic and Applied Research 2015;23(2):156-63. [gssrr.org/index.php? journal=JournalOfBasicAndApplied\&page=article\&op=view\&path \%5B\%5D=4329\&path\%5B\%5D=2383]

\section{Muslihah 2016 \{published data only\}}

Muslihah N, Khomsan A, Briawan D, Riyadi H. Effect of the provision of small-quantity lipid-based nutrient supplements on gross motor developmental milestones in Indonesian infants. Pakistan Journal of Nutrition 2016;15(9):889-96. [DOI: 10.3923/pjn.2016.889.896]

\section{Rantesalu 2017 \{published data only\}}

Rantesalu M. Effect of a lipid-based nutrient supplement on insulin-like growth factor-1 level (IGF-1) in 6-12 month-old children in South Central Timor of the East Nusa Tenggara Province. Pakistan Journal of Nutrition 2017;16(6):417-25. [DOI: 10.3923/pjn.2017.417.425]

\section{Schlossman 2017 \{published data only\}}

Schlossman N, Brown C, Batra P, de Sa AB, Balan I, Balan A, et al. A randomized controlled trial of two ready-to-use supplementary foods demonstrates benefit of the higher dairy supplement for reduced wasting in mothers, and differential impact in infants and children associated with maternal supplement response. Food and Nutrition Bulletin 2017;38(3):275-90. [DOI: 10.1177/0379572117700754; PUBMED: 28374648]

\section{Style 2017 \{published data only\}}

Style S, Tondeur M, Grijalva-Eternod C, Pringle J, Kassim I, Wilkinson C, et al. Assessment of the effectiveness of a small quantity lipid-based nutrient supplement on reducing anaemia and stunting in refugee populations in the Horn of Africa: secondary data analysis. PLOS One 2017;12(6):e0177556. [DOI: 10.1371/journal.pone.0177556; PMC5462343; PUBMED: 28591166]

Thakwalakwa 2010 \{published data only\}

Thakwalakwa C, Ashorn P, Phuka J, Cheung YB, Briend A, Puumalainen T, et al. A lipid-based nutrient supplement but not corn-soy blend modestly increases weight gain among 6- to 18 month-old moderately underweight children in rural Malawi. Journal of Nutrition 2010;140(11):2008-13. [DOI: 10.3945/ jn.110.122499; PUBMED: 20861218]

\section{Thakwalakwa 2012 \{published data only\}}

Thakwalakwa CM, Ashorn P, Jawati M, Phuka JC, Cheung YB, Maleta KM. An effectiveness trial showed lipid-based nutrient supplementation but not corn-soya blend offered a modest benefit in weight gain among 6-to 18-month-old underweight children in rural Malawi. Public Health Nutrition 2012;15(9):1755-62. [DOI: 10.1017/S1368980012003023; PUBMED: 22691922]

\section{Thakwalakwa 2015 \{published data only\}}

Thakwalakwa CM, Ashorn P, Phuka JC, Cheung YB, Briend A, Maleta KM. Impact of lipid-based nutrient supplements and corn-soy blend on energy and nutrient intake among moderately underweight 8-18-month-old children participating

Preventive lipid-based nutrient supplements given with complementary foods to infants and young children 6 to 23 months of age for 
in a clinical trial. Maternal \& Child Nutrition 2015;11(Suppl 4):144-50. [DOI: 10.1111/mcn.12105; PUBMED: 24528807]

\section{Unger 2017 \{published data only\}}

Unger SA, Drammeh S, Hasan J, Ceesay K, Sinjanka E, Beyai $S$, et al. Impact of fortified versus unfortified lipidbased supplements on morbidity and nutritional status: a randomised double-blind placebo-controlled trial in ill Gambian children. PLOS Medicine 2017;14(8):e1002377. [DOI: 10.1371/journal.pmed.1002377; PMC5557358; PUBMED: 28809926]

\section{Vargas-Vásquez 2015 \{published data only\}}

Vargas-Vásquez A, Bado R, Alcázar L, Aquino O, Rodríguez A, Novalbos JP. Effects of a lipid-based nutrient supplement on hemoglobin levels and anthropometric indicators in children from five districts in Huánuco Peru [Efecto de un suplemento nutricional a base de lípidos en los niveles de hemoglobina e indicadores antropométricos en niños de cinco distritos de Huánuco, Perú]. Revista Peruana de Medicina Experimental y Salud Pública 2015;32(2):237-44. [PUBMED: 26338380]

\section{References to ongoing studies}

\section{Borg 2017 \{published data only\}}

Borg B, Mihrshahi S, Griffin M, Chamnan C, Laillou A, Wieringa FT. Crossover trial to test the acceptability of a locally produced lipid-based nutrient supplement (LNS) for children under 2 years in Cambodia: a study protocol. BMJ Open 2017;7(9):e015958. [DOI: 10.1136/bmjopen-2017-015958]

\section{Fernald 2016 \{published data only\}}

Fernald LC, Galasso E, Qamruddin J, Ranaivoson C, Ratsifandrihamanana L, Stewart CP, et al. A cluster-randomized, controlled trial of nutritional supplementation and promotion of responsive parenting in Madagascar: the MAHAY study design and rationale. BMC Public Health 2016;16:466. [10.1186/ s12889-016-3097-7]

\section{Huybregts 2017 \{published data only\}}

Huybregts L, Becquey E, Zongrone A, Le Port A, Khassanova R, Coulibaly $L$, et al. The impact of integrated prevention and treatment on child malnutrition and health: the PROMIS project, a randomized control trial in Burkina Faso and Mali. BMC Public Health 2017;17(1):237. [DOI: 10.1186/ s12889-017-4146-6; PMC5343313; PUBMED: 28274214]

\section{ISRCTN94319790 \{published data only\}}

ISRCTN94319790. Ready to Use Supplementary Foods (RUSF) to prevent stunting among children under five years in Kurram Agency [A community-based cluster controlled trial to evaluate the effectiveness of Ready to Use Supplementary Foods (RUSF) and proportional contribution of multi-sectoral interventions in the prevention of stunting among children under five years in Kurram Agency, Pakistan]. apps.who.int/trialsearch/Trial2.aspx? TrialID=ISRCTN94319790 (first received 11 December 2017).

\section{SHINE trial 2015 \{published data only\}}

Sanitation Hygiene Infant Nutrition Efficacy (SHINE) Trial Team, Humphrey JH, Jones AD, Manges A, Mangwadu G, Maluccio JA, Mbuya MN, et al. The Sanitation Hygiene Infant Nutrition
Efficacy (SHINE) trial: rationale, design, and methods. Clinical Infectious Diseases 2015;61(Supp 7):S685-702. [DOI: 10.1093/ cid/civ844; PMC4657589; PUBMED: 26602296]

\section{Additional references}

\section{Adu-Afarwuah 2011}

Adu-Afarwuah S, Lartey A, Zeilani M, Dewey KG. Acceptability of lipid-based nutrient supplements (LNS) among Ghanaian infants and pregnant or lactating women. Maternal \& Child Nutrition 2011;7(4):344-56. [DOI: 10.1111/ j.1740-8709.2010.00286.x; PUBMED: 21496207]

\section{Arimond 2015}

Arimond M, Zeilani M, Jungjohann S, Brown KH, Ashorn P, Allen $\mathrm{LH}$, et al. Considerations in developing lipid-based nutrient supplements for prevention of undernutrition: experience from the International Lipid-Based Nutrient Supplements (iLiNS) Project. Maternal \& Child Nutrition 2015;11 Suppl 4:31-61. [DOI: 10.1111/mcn.12049; PUBMED: 23647784]

\section{Balshem 2010}

Balshem H, Helfanda M, Schünemann HJ, Oxman AD, Kunze R, Brozek J, et al. GRADE guidelines: 3: rating the quality of evidence. Journal of Clinical Epidemiology 2010;64(4):401-6. [DOI: 10.1016/j.jclinepi.2010.07.015; PUBMED: 21208779]

\section{Bendabenda 2016}

Bendabenda J, Alho L, Ashorn U, Cheung YB, Dewey KG, Vosti SA, et al. The effect of providing lipid-based nutrient supplements on morbidity in rural Malawian infants and young children: a randomized controlled trial. Public Health Nutrition 2016;19(10):1893-903. [DOI: 10.1017/S1368980016000331; PUBMED: 26956611]

\section{Bhutta 2013}

Bhutta ZA, Das JK, Rizvi A, Gaffey MF, Walker N, Horton S, et al. Evidence based interventions for improvement of maternal and child nutrition: what can be done and at what cost?. Lancet 2013;382(9890):452-77. [DOI: 10.1016/S0140-6736(13)60996-4; PUBMED: 23746776]

\section{Black 2013}

Black RE, Victora CG, Walker SP, Bhutta ZA, Christian P, de Onis $\mathrm{M}$, et al. Maternal and child undernutrition and overweight in low-income and middle-income countries. Lancet 2013;382(9890):427-51. [DOI: 10.1016/S0140-6736(13)60937-X; PUBMED: 23746772]

\section{Chaparro 2010}

Chaparro CM, Dewey KG. Use of lipid-based nutrient supplements (LNS) to improve the nutrient adequacy of general food distribution rations for vulnerable sub-groups in emergency settings. Maternal \& Child Nutrition 2010;6(Suppl 1):1-69. [DOI: 10.1111/j.1740-8709.2009.00224.x; PUBMED: 20055936]

\section{Das 2018}

Das JK, Hoodbhoy Z, Salam RA, Bhutta AZ, ValenzuelaRubio NG, Weise Prinzo Z, et al. Lipid-based nutrient supplements for maternal, birth, and infant developmental

Preventive lipid-based nutrient supplements given with complementary foods to infants and young children 6 to 23 months of age for health, nutrition, and developmental outcomes (Review)

Copyright (c) 2019 The Cochrane Collaboration. Published by John Wiley \& Sons, Ltd. 
outcomes. Cochrane Database of Systematic Reviews 2018, Issue 8. [DOI: 10.1002/14651858.CD012610.pub2; PUBMED: 30168868]

\section{De-Regil 2013}

De-Regil LM, Suchdev PS, Vist GE, Walleser S, Peña-Rosas JP. Home fortification of foods with multiple micronutrient powders for health and nutrition in children under two years of age (Review). Evidence-Based Child Health: A Cochrane Review Journal 2013;8(1):112-201. [DOI: 10.1002/ebch.1895]

\section{Deeks 2011}

Deeks J, Higgins JP, Altman D on behalf of the Cochrane Statistical Methods Group. Chapter 9: Analysing data and undertaking meta-analyses. In: Higgins JP, Green S (editors). Cochrane Handbook for Systematic Reviews of Interventions Version 5.1.0 (updated March 2011). The Cochrane Collaboration, 2011. Available from handbook.cochrane.org.

\section{Dewey 2012}

Dewey KG, Arimond M. Lipid-based nutrient supplements: how can they combat child malnutrition?. PLOS Medicine 2012;9(9):e1001314. [DOI: 10.1371/journal.pmed.1001314; PMC3445442; PUBMED: 23028264]

\section{Dewey 2016 [pers comm]}

Dewey KG. RE: Preliminary results of LNS reviews [personal communication]. Email to: ZA Bhutta 14 November 2016.

\section{Dewey 2017 [pers comm]}

Dewey KG. Another iLiNS-ZINC paper [personal communication]. Email to: ZA Bhutta 04 February 2017.

\section{Egger 1997}

Egger M, Davey Smith G, Schneider M, Minder C. Bias in meta-analysis detected by a simple, graphical test. BMJ 1997;315(7109):629-34. [DOI: 10.1136/bmj.315.7109.629; PMC2127453; PUBMED: 9310563]

\section{GRADEpro GDT 2015 [Computer program]}

McMaster University (developed by Evidence Prime). GRADEpro GDT. Version accessed 29 April 2016. Hamilton (ON): McMaster University (developed by Evidence Prime), 2015.

\section{Harbord 2006}

Harbord RM, Egger M, Sterne JA. A modified test for smallstudy effects in meta-analyses of controlled trials with binary endpoints. Statistics in Medicine 2006;25(20):3443-57. [DOI: 10.1002/sim.2380; PUBMED: 16345038$]$

\section{Hess 2011}

Hess SY, Bado L, Aaron GJ, Ouédraogo JB, Zeilani M, Brown KH. Acceptability of zinc-fortified, lipid-based nutrient supplements (LNS) prepared for young children in Burkina Faso. Maternal \& Child Nutrition 2011;7(4):357-67. [DOI: 10.1111/ j.1740-8709.2010.00287.x; PUBMED: 21159124]

\section{Higgins 2011a}

Higgins JP, Altman DG, Sterne JA. Chapter 8: Assessing risk of bias in included studies. In: Higgins JP, Green S, editor(s). Cochrane Handbook for Systematic Reviews of
Interventions Version 5.1 .0 (updated March 2011). The Cochrane Collaboration, 2011. Available from handbook.cochrane.org.

\section{Higgins 2011b}

Higgins JP, Deeks JJ, Altman DG. Chapter 16: Special topics in statistics. In: Higgins JP, Green S, editor(s). Cochrane Handbook for Systematic Reviews of Interventions Version 5.1.0 (updated March 2011). The Cochrane Collaboration, 2011. Available from handbook.cochrane.org.

\section{lannotti 2013}

Iannotti LL, Dulience SJ, Green J, Joseph S, François J, Anténor ML, et al. Linear growth increased in young children in an urban slum of Haiti: a randomized controlled trial of a lipid-based nutrient supplement. Amercian Journal of Clinical Nutrition 2013;99(1):198-208. [DOI: 10.3945/ajcn.113.063883]

\section{Ilins 2015}

iLiNS. iLiNS Project. ilins.org/resources (accessed 12 January 2016).

\section{Kristjansson 2015}

Kristjansson E, Francis DK, Liberato S, Benkhalti Jandu M, Welch V, Batal M, et al. Food supplementation for improving the physical and psychosocial health of socio-economically disadvantaged children aged three months to five years. Cochrane Database of Systematic Reviews 2015, Issue 3. [DOI: 10.1002/14651858.CD009924.pub2; PUBMED: 25739460]

\section{Kristjansson 2016}

Kristjansson E, Francis D, Liberato S, Greenhalgh T, Welch V, Jandu MB, et al. Supplementary Feeding for Improving the Health of Disadvantaged Infants and Children: What Works and Why? Systematic Review Summary 5. London (UK): International Initiative for Impact Evaluation (3ie), 2016.

\section{Larson 2017}

Larson LM, Yousafzai AK. A meta-analysis of nutrition interventions on mental development of children under-two in low- and middle-income countries. Maternal \& Child Nutrition 2017;13(1):e12229. [DOI: 10.1111/mcn.12229; PUBMED: 26607403]

\section{Lassi 2013}

Lassi ZS, Das JK, Zahid G, Imdad A, Bhutta ZA. Impact of education and provision of complementary feeding on growth and morbidity in children less than 2 years of age in developing countries: a systematic review. BMC Public Health 2013;13(Suppl 3):S13. [DOI: 10.1186/1471-2458-13-S3-S13; PMC3847349; PUBMED: 24564534]

\section{Liu 2012}

Liu L, Johnson HL, Cousens S, Perin J, Scott S, Lawn JE, et al. Global, regional, and national causes of child mortality: an updated systematic analysis for 2010 with time trends since 2000. Lancet 2012;379(9832):2151-61. [DOI: 10.1016/ S0140-6736(12)60560-1]

\section{Maleta 2015}

Maleta KM, Phuka J, Alho L, Cheung YB, Dewey KG, Ashorn U, et al. Provision of $10-40 \mathrm{~g} / \mathrm{d}$ lipid-based nutrient supplements 
from 6 to 18 months of age does not prevent linear growth faltering in Malawi. Journal of Nutrition 2015;145(8):1909-15. [DOI: 10.3945/jn.114.208181; PUBMED: 26063066]

\section{Mangani 2013}

Mangani C, Cheung YB, Maleta K, Phuka J, Thakwalakwa C, Dewey $\mathrm{K}$, et al. Providing lipid-based nutrient supplements does not affect developmental milestones among Malawian children. Acta Paediatrica 2013;103(1):e17-26. [DOI: 10.1111/apa.12443; PUBMED: 24118040]

\section{Moher 2009}

Moher D, Liberati A, Tetzlaff J, Altman DG. Preferred reporting items for systematic reviews and meta-analyses: the PRISMA statement. BMJ 2009;339:b2535. [DOI: 10.1136/bmj.b2535]

\section{Pee 2008}

Pee S, Bloem MW. Current and potential role of specially formulated foods and food supplements for preventing malnutrition among 6-23 months old and treating moderate malnutrition among 6-59 months old children. www.who.int/ nutrition/publications/moderate_malnutrition/MM_ Background_paper4.pdf (accessed 14 December 2016).

\section{Prado 2016}

Prado EL, Maleta K, Ashorn P, Ashorn U, Vosti SA, Sadalaki J, et al. Effects of maternal and child lipid-based nutrient supplements on infant development: a randomized trial in Malawi. American Journal of Clinical Nutrition 2016;103(3):784-93. [DOI: 10.3945/ajcn.115.114579; PUBMED: 26843155]

\section{Review Manager 2014 [Computer program]}

Nordic Cochrane Centre, The Cochrane Collaboration. Review Manager 5 (RevMan 5). Version 5.3. Copenhagen: Nordic Cochrane Centre, The Cochrane Collaboration, 2014.

\section{Segrè 2015}

Segrè J, Winnard K, Abrha TH, Abebe Y, Shilane D, Lapping K. Willingness to pay for lipid-based nutrient supplements for young children in four urban sites of Ethiopia. Maternal \& Child Nutrition 2015;11(Suppl 4):16-30. [DOI: 10.1111/mcn.12022; PUBMED: 23241477]

\section{Sguassero 2012}

Sguassero Y, de Onis M, Bonotti AM, Carroli G. Communitybased supplementary feeding for promoting the growth of children under five years of age in low and middle income countries. Cochrane Database of Systematic Reviews 2012, Issue 6. [DOI: 10.1002/14651858.CD005039.pub3; PUBMED: 22696347]

\section{Stewart 2017 [pers comm]}

Stewart CP. RE: [personal communication]. Email to: ZA Bhutta 27 April 2017.

\section{The World Bank 2006}

The World Bank. Repositioning Nutrition as Central to Development: A Strategy for Large Scale Action. Overview. Washington (DC): The World Bank, 2006. [siteresources.worldbank.org/
NUTRITION/Resources/281846-1131636806329/

NutritionStrategyOverview.pdf]

\section{UNICEF 2013}

UNICEF, CDC. Global assessment of home fortification interventions, 2011: final report May 2013. www.hftag.org/ resource/global-assessment-of-home-fortificationinterventions-2011-pdf/ (accessed 21 November 2015).

\section{UNICEF 2014}

UNICEF. Humanitarian action for children 2014: overview. www.unicef.org/gambia/Humanitarian_Action_for_Childen_ 2014_Overview.pdf (accessed 22 November 2015).

\section{Victora 2008}

Victora CG, Adair L, Fall C, Hallal PC, Martorell R, Richter L, et al. Maternal and child undernutrition: consequences for adult health and human capital. Lancet 2008;371(9609):340-57. [DOI: 10.1016/S0140-6736(07)61692-4]

\section{WHO 2011}

World Health Organization. Guideline: use of multiple micronutrient powders for home fortification of foods consumed by infants and children 6-23 months of age. apps.who.int/iris/ bitstream/10665/44651/1/9789241502047_eng.pdf (accessed 25 November 2015).

\section{WHO 2012}

WHO. Technical Note: Supplementary Foods for the Management of Moderate Acute Malnutrition in Infants and Children 6-59 Months of Age. Geneva $(\mathrm{CH})$ : World Health Organization, 2012. [apps.who.int/iris/ bitstream/10665/75836/1/9789241504423_eng.pdf]

\section{WHO 2013}

World Health Organization. Guideline: updates on the management of severe acute malnutrition in infants and children. apps.who.int/iris/ bitstream/10665/95584/1/9789241506328_eng.pdf (accessed 4 December 2015).

\section{WHO 2014}

World Health Organization. WHA global nutrition targets 2025: stunting policy brief. www.who.int/nutrition/topics/ globaltargets_stunting_policybrief.pdf (accessed 4 January 2016).

\section{Wisner 2002}

Wisner B, Adams J, editor(s). Environmental Health in Emergencies and Disasters: A Practical Guide. Geneva (CH): World Health Organization, 2002. [apps.who.int/ iris/bitstream/handle/10665/42561/9241545410_ eng.pdf;jsessionid=335412CBA6A98678D7D9E0C9017D2D1E? sequence $=1$ ]

\section{References to other published versions of this review \\ Das 2017}

Das JK, Salam RA, Weise Prinzo Z, Sadiq Sheikh S, Bhutta ZA. Provision of preventive lipid-based nutrient supplements given

Preventive lipid-based nutrient supplements given with complementary foods to infants and young children 6 to 23 months of age for 
with complementary foods to infants and young children 6 to 23 months of age for health, nutrition, and developmental outcomes. Cochrane Database of Systematic Reviews 2017, Issue 3. [DOI: 10.1002/14651858.CD012611]

\section{Das 2019}

Das JK, Salam RA, Weise Prinzo Z, Sadiq Sheikh S, Bhutta ZA. Provision of preventive lipid-based nutrient supplements given

\section{CHARACTERISTICS OF STUDIES}

Characteristics of included studies [ordered by study ID]

Adu-Afarwuah 2007

Methods Design: community-based randomised controlled trial involving 3 intervention groups and 1 nonintervention (NI) group.

Unit of randomisation: individual

Location/Setting: Koforidua, in the Eastern Region of Ghana
Sample size: 409 Ghanian infants (5 months of age)
Dropouts/withdrawals: 15 lost to follow-up
Sex: both male and female children included
Mean age: children enrolled at 5 months of age
Inclusion criteria
1. 5 months of age
2. Receiving any breast milk
3. Not known to be asthmatic or allergic to peanuts
4. Planning to stay at the study site during the next 7 months

Exclusion criteria: not specified with complementary foods to infants and young children 6 to 23 months of age for health, nutrition, and developmental outcomes. Cochrane Database of Systematic Reviews 2019, Issue 3. [DOI: 10.1002/14651858.CD012611.pub2]

* Indicates the major publication for the study

The infants were randomly assigned to receive either of the following until 12 months of age.

\section{Intervention}

1. Sprinkles (SP) powder $(n=105)$

2. crushable Nutritabs (NT) tablets $(n=105)$

3. Energy-dense (108 kcal/day), fat-based Nutributter (NB) $(n=103)$

Control: no intervention $(n=96)$

\section{Primary outcomes}

1. Weight;

2. Head circumference

3. Length-for age $z$ score (LAZ)

4. Weight-for-age z score (WAZ)

5. Weight-for-length z score (WLZ)

\section{Secondary outcomes}

1. Diarrhoea

2. Fever

3. Upper respiratory tract infection 
Adu-Afarwuah 2007 (Continued)

Timing of outcome assessment: at 12 months of age

Study start date: February 2004
Study end date: June 2005
Funding source: Nutriset manufactured NB.
Conflicts of interest: one of the study authors was a paid consultant of Nutriset. None of the other
study authors had any potential conflicts of interest.

Comment: none

\section{Risk of bias}

Bias Authors' judgement Support for judgement

Random sequence genera- High risk

Quote: "we randomly selected $75 \%$ of eligible infants by entering identification numbers for all eligible infants in a dataset and using a SAS data step....The NI infants were enrolled from among the $25 \%$ of those who were originally eligible but not randomly selected for the intervention groups"

Comment: not adequate

\begin{tabular}{ll}
\hline $\begin{array}{l}\text { Allocation concealment } \\
\text { (selection bias) }\end{array}$ & Low risk \\
& $\begin{array}{l}\text { Quote: "the infants were randomly assigned (with the use of opaque en- } \\
\text { velopes with group designations) to receive SP, NT, or NB until } 12 \text { mo of age" } \\
\text { Comment: adequately done }\end{array}$ \\
\hline
\end{tabular}

\begin{tabular}{|c|c|c|}
\hline $\begin{array}{l}\text { Blinding of participants } \\
\text { and personnel (perfor- } \\
\text { mance bias) }\end{array}$ & High risk & $\begin{array}{l}\text { Quote: "NT was provided to the mothers in plastic bags, and the NB }(20 \mathrm{~g} / \mathrm{d}) \\
\text { was provided in foil packs with screw caps" }\end{array}$ \\
\hline All outcomes & & Comment: not adequate \\
\hline
\end{tabular}

\begin{tabular}{lll}
\hline $\begin{array}{l}\text { Blinding of outcome as- } \\
\text { sessment (detection bias) } \\
\text { All outcomes }\end{array}$ & Unclear risk & Comment: not clearly specified \\
\hline $\begin{array}{l}\text { Incomplete outcome data } \\
\text { (attrition bias) } \\
\text { All outcomes }\end{array}$ & Low risk & Attrition: SP group $=98 / 105 ;$ NT group $=102 / 105 ;$ NB group $=98 / 103$ \\
\hline $\begin{array}{l}\text { Selective reporting (re- } \\
\text { porting bias) }\end{array}$ & Low risk & Comment: reasons provided for loss to follow-up \\
\hline
\end{tabular}

Other bias Low risk Comment: no other potential sources of bias reported

\section{Adu-Afarwuah 2016}

Methods Design: a partially double-blind, parallel, randomised, controlled trial with 3 equal-size groups.

Unit of randomisation: individual

Participants

Location/Setting: the study was conducted in several adjoining semi-urban communities (Somany-Odumasi-Kpong area) in the Yilo Krobo and the Lower Manya Krobo districts in Ghana.

Sample size: 1320 women at $<20$ weeks of gestation, and 1228 infants 
Sex: both male and female children included

Mean age: children were enrolled at birth and the intervention was started at 6 months of age.

Inclusion criteria: pregnant women attending usual antenatal clinics in the 4 main health facilities in the area were included if they were:

1. $>18$ years old;

2. at $<20$ weeks of gestation based on the information available at the time.

\section{Exclusion criteria}

1. Not residing in the area

2. Intention to move within the next 2 years

3. Milk or peanut allergy

4. Participation in another trial

5. HIV infection

6. Asthma

7. Epilepsy

8. Tuberculosis

9. Any malignancy

10.Unwillingness to sign or thumbprint the relevant consent forms, receive field workers, or take the study supplement

Interventions

Intervention

1. MMN group ( $n=439$ women and 411 infants)

2. SQ LNS (LNS group) ( $n=440$ women and 409 infants)

Control: IFA group ( $\mathrm{n}=441$ women and 408 infants)

Interventions were provided to mothers until delivery, and then either placebo, MMN, or SQ LNS for 6 months postpartum. Infants in the LNS group received SQ LNS formulated for infants from 6 to 18 months of age (endline). SQ LNS provided $20 \mathrm{~g} /$ day or $118 \mathrm{kcal}$.

Outcomes

\section{Primary outcomes}

1. Length

2. Length-for age $z$ score (LAZ)

\section{Secondary outcomes}

1. Weight

2. Mid-upper arm circumference (MUAC)

3. Head circumference

4. Weight-for-age z score (WAZ)

5. Weight-for-length z score (WLZ)

6. Stunting

7. Underweight

8. Wasting

9. Hospitalisation

10.Deaths

Timing of outcome assessment: at 18 months of age 
Adu-Afarwuah 2016 (Continued)

Study end date: December 2011

Funding source: Bill \& Melinda Gates Foundation. The funder had no role in the design of the study; data collection, analysis, and interpretation; or in the preparation of the manuscript.

Conflicts of interest: one of the study authors was an employee of Nutriset SAS, which is a commercial producer of LNS products. The study authors declared that they had no conflicts of interest related to this study.

Comment: none

\section{Risk of bias}

\begin{tabular}{lll}
\hline Bias & Authors' judgement & Support for judgement \\
\hline $\begin{array}{l}\text { Random sequence genera- } \\
\text { tion (selection bias) }\end{array}$ & Low risk & $\begin{array}{l}\text { Quote: "The study statistician at University of California, Davis developed } \\
\text { group allocations with the use of a computer-generated (SAS version 9.3; SAS } \\
\text { Institute) randomization scheme in blocks of 9" }\end{array}$
\end{tabular}

Comment: adequately done

Allocation concealment Low risk
(selection bias)

Quote: "At each enrollment, the study nurse offered sealed, opaque envelopes bearing group allocations, 9 envelopes at a time, and the woman picked one to reveal the allocation"

Comment: adequately done

\begin{tabular}{|c|c|c|}
\hline $\begin{array}{l}\text { Blinding of participants } \\
\text { and personnel (perfor- } \\
\text { mance bias) } \\
\text { All outcomes }\end{array}$ & High risk & $\begin{array}{l}\text { Quote: "It was not possible to blind study workers and participants to the cap- } \\
\text { sules (IFA and MMN supplements) compared with the LNS supplements be- } \\
\text { cause of their apparent differences, but laboratory staff, anthropometrists, } \\
\text { and data analysts had no knowledge of group assignment until all preliminary } \\
\text { analyses had been completed" }\end{array}$ \\
\hline
\end{tabular}

Comment: not adequate

\begin{tabular}{lll}
\hline $\begin{array}{l}\text { Blinding of outcome as- } \\
\text { sessment (detection bias) }\end{array}$ & Low risk & $\begin{array}{l}\text { Quote: "It was not possible to blind study workers and participants to the cap- } \\
\text { sules (IFA and MMN supplements) compared with the LNS supplements be- } \\
\text { cll outcomes }\end{array}$ \\
& $\begin{array}{l}\text { cause of their apparent differences, but laboratory staff, anthropometrists, } \\
\text { and data analysts had no knowledge of group assignment until all preliminary } \\
\text { analyses had been completed" }\end{array}$
\end{tabular}

Comment: adequately done

\begin{tabular}{lll}
\hline $\begin{array}{l}\text { Incomplete outcome data } \\
\text { (attrition bias) } \\
\text { All outcomes }\end{array}$ & Low risk & Attrition: IFA group = 393/408; MMN group = 401/411; LNS group = 391/409 \\
\hline $\begin{array}{l}\text { Selective reporting (re- } \\
\text { porting bias) }\end{array}$ & Low risk & $\begin{array}{l}\text { Comment: trial registered as NCT00970866 at ClinicalTrials.gov. Outcomes de- } \\
\text { scribed in the protocol and methodology section reported in the results sec- } \\
\text { tion }\end{array}$
\end{tabular}

\begin{tabular}{ll}
\hline Other bias $\quad$ Low risk $\quad$ Comment: no other potential sources of bias reported \\
\hline
\end{tabular}

\section{Ashorn 2015}

Methods Design: randomised, controlled, outcome-assessor-blinded trial

Unit of randomisation: individual 
Ashorn 2015 (Continued)

Participants
Location/Setting: 1 public district hospital (Mangochi), 1 rural, semi-private hospital (Malindi), and 1 rural public health centre (Lungwena) in Mangochi District, Southern Malawi

Sample size: 869 pregnant women and their 781 children were enrolled.

Dropouts/withdrawals: 22 lost to follow-up

Sex: both male and female children included

Mean age: children were enrolled at birth and the intervention was started at 6 months of age

\section{Inclusion criteria}

1. Ultrasound scan confirmed pregnancy of \#20 completed gestation weeks

2. Residence in the defined catchment area

3. Availability during the period of the study

4. Signed or thumb-printed informed consent

\section{Exclusion criteria}

1. $<15$ years of age

2. Need for frequent medical attention due to a chronic health condition

3. Diagnosed asthma treated with regular medication

4. Severe illness that warranted hospital referral

5. History of allergy to peanuts

6. History of anaphylaxis or serious allergic reaction to any substance that required emergency medical care

7. Pregnancy complications evident at the enrolment visit

8. Earlier participation in the iLiNS-DYAD-M trial (during a previous pregnancy), or concurrent participation in any other clinical trial

\section{Intervention (during pregnancy and 6 months thereafter)}

1. Women $(n=291)$ received 1 capsule containing 18 MMN daily, children $(n=233)$ received no supplement

2. Women $(n=288)$ received $120 \mathrm{~g}$ sachet of SQ LNS daily (containing $21 \mathrm{MMN}$, protein, carbohydrates, essential fatty acids, and $118 \mathrm{kcal})$, children $(n=222)$ received SQ LNS from 6 to 18 months

Control (during pregnancy and 6 months thereafter): women $(n=290)$ received 1 capsule of IFA daily, children $(n=223)$ received no supplement

Outcomes

\section{Primary outcome}

1. length

\section{Secondary outcomes}

1. Weight

2. Length for age $z$ score (LAZ)

3. Weight for age $z$ score (WAZ)

4. weight for height $\mathrm{z}$ score (WHZ)

5. Head circumference and corresponding $z$ score

6. Arm circumference and corresponding z score

7. Stunting

8. Underweight

9. Wasting

Timing of outcome assessment: at 18 months of age 
Ashorn 2015 (Continued)

Notes
Study start date: February 2011

Study end date: August 2012

Funding source: supported, in part, by a grant to the University of California, Davis, from the Bill \& Melinda Gates Foundation, with additional funding from the Office of Health, Infectious Diseases, and Nutrition, Bureau for Global Health, USAID under terms of co-operative agreement AID-OAAA-12-00005, through the FANTA project, managed by FHI 360.

Conflicts of interest: one of the study authors works as a director of research for Nutriset SAS, a company that produces and sells LNS and which also prepared the LNS purchased for the present trial. All other study authors declared no conflict of interest.

Comment: SQ LNS was provided to mothers in pregnancy and 6 months postpartum, and to their infants from 6 to 18 months of age.

\section{Risk of bias}

\begin{tabular}{lll} 
Bias & Authors' judgement & Support for judgement \\
\hline $\begin{array}{ll}\text { Random sequence genera- } \\
\text { tion (selection bias) }\end{array}$ & Low risk & $\begin{array}{l}\text { Quote: "Researcher not involved with the trial created individual randomisa- } \\
\text { tion slips (in blocks of 9) and packed them in sealed, numbered, opaque ran- } \\
\text { domisation envelopes that were stored in numerical order" }\end{array}$
\end{tabular}

Comment: adequately done

\begin{tabular}{ll}
\hline $\begin{array}{l}\text { Allocation concealment } \\
\text { (selection bias) }\end{array}$ & Low risk \\
& $\begin{array}{l}\text { Quote: "Eligible pregnant women were requested to choose } 1 \text { of the top } 6 \text { en- } \\
\text { velopes in the stack, and the contents of the envelope indicated her partici- } \\
\text { pant number and group allocation" }\end{array}$
\end{tabular}

Comment: adequately done

\begin{tabular}{|c|c|c|}
\hline $\begin{array}{l}\text { Blinding of participants } \\
\text { and personnel (perfor- } \\
\text { mance bias) } \\
\text { All outcomes }\end{array}$ & High risk & $\begin{array}{l}\text { Quote: "we used single masked procedures; that is, field workers who deliv- } \\
\text { ered the supplements knew which mothers were receiving LNS, and the par- } \\
\text { ticipants were advised not to disclose information about their supplements to } \\
\text { anyone other than an iLiNS team member" }\end{array}$ \\
\hline
\end{tabular}

Comment: not done

\begin{tabular}{|c|c|c|}
\hline $\begin{array}{l}\text { Blinding of outcome as- } \\
\text { sessment (detection bias) } \\
\text { All outcomes }\end{array}$ & Low risk & $\begin{array}{l}\text { Quote: "The data collectors who performed the anthropometric measure- } \\
\text { ments or assessed other outcomes were not aware of group allocation. Re- } \\
\text { searchers responsible for the data cleaning remained blind to the trial code, } \\
\text { until the database was considered fully cleaned" }\end{array}$ \\
\hline
\end{tabular}

Comment: adequately done

\begin{tabular}{lll}
\hline $\begin{array}{l}\text { Incomplete outcome data } \\
\text { (attrition bias) } \\
\text { All outcomes }\end{array}$ & Low risk & $\begin{array}{l}\text { Attrition: } \text { IFA group }=220 / 223 ; \text { MMN group }=222 / 233 ; \text { LNS group }=214 / 222 \\
\text { Comment: } \text { reasons for loss to follow-up mentioned }\end{array}$ \\
\hline $\begin{array}{l}\text { Selective reporting (re- } \\
\text { porting bias) }\end{array}$ & Low risk & $\begin{array}{l}\text { Comment: trial registered as NCT01239693 at ClinicalTrials.gov. Published } \\
\text { protocol reviewed }\end{array}$ \\
\hline Other bias & Low risk & Comment: no other potential sources of bias reported \\
\hline
\end{tabular}

Bisimwa 2012

Methods Design: a prospective, non-blinded, randomised, controlled, parallel-group trial.


Bisimwa 2012 (Continued)

Unit of randomisation: individual

Participants Location/Setting: Lwiro Pediatric Hospital, Miti Murhesa Health District, South Kivu Province, Democratic Republic of Congo

Sample size: 1383 children (6 months of age)

Dropouts/withdrawals: 52 lost to follow-up

Sex: both male and female children included

Mean age: children were recruited at birth and the intervention initiated at 6 months of age.

Inclusion criteria: full-term-born infants (gestational age $>37$ weeks) were recruited when they were 4-5 months of age, if consent was given.

\section{Exclusion criteria}

1. Exclusively bottle-fed children

2. Children with any malformations or neurologic impairment

\begin{tabular}{ll}
\hline Interventions & Intervention: lipid-based RUCF $(\mathrm{n}=691)$ \\
Control: UNIMIX $(\mathrm{n}=692)$ & Primary outcome \\
\hline Outcomes & Stunting \\
Secondary outcomes \\
1. Underweight \\
2. Weight \\
3. Length \\
4. Mid-upper arm circumference (MUAC) \\
5. Length-for-age $z$ score (LAZ) \\
6. Weight-for-age $\mathrm{z}$ score (WAZ) \\
7. Weight-for-length z score (WLZ) \\
8. Mean changes in hematocrit, cholesterol, and triglyceride concentrations \\
9. Proportion of children walking unassisted (as reported by mother/caregiver) at 12 months of age \\
Timing of outcome assessment: at 12 months of age
\end{tabular}

Notes

\section{Study start date: October 2009}

Study end date: November 2010

Funding source: supported by funds from Irish Aid, Department of Foreign Affairs, Republic of Ireland.

Conflicts of interest: three study authors work for Valid Nutrition, who developed the RUCF. One study author worked for Valid International, which is Valid Nutrition's sister company. All other study authors had no conflicts of interest.

Comment: none

\section{Risk of bias}

\begin{tabular}{lll}
\hline Bias & Authors' judgement & Support for judgement \\
\hline $\begin{array}{l}\text { Random sequence genera- } \\
\text { tion (selection bias) }\end{array}$ & Low risk & $\begin{array}{l}\text { Quote: "A computer program, with the use of a random-number generator } \\
\text { function, created lists for each study team that comprised 2 trained enumera- } \\
\text { tors" }\end{array}$ \\
\hline
\end{tabular}

Preventive lipid-based nutrient supplements given with complementary foods to infants and young children 6 to 23 months of age for 
Bisimwa 2012 (Continued)

Comment: adequately done

\begin{tabular}{ll}
\hline $\begin{array}{l}\text { Allocation concealment } \\
\text { (selection bias) }\end{array}$ & $\begin{array}{l}\text { Quote: "On the basis of the generated randomization lists, the field supervisor } \\
\text { who was not involved in the recruitment of participants prepared sealed en- } \\
\text { velopes labelled with consecutive numbers, with each of the envelopes con- } \\
\text { taining the code of the study food that was to be assigned" } \\
\text { Comment: adequately done }\end{array}$
\end{tabular}

\begin{tabular}{ll}
\hline $\begin{array}{l}\text { Blinding of participants } \\
\text { and personnel (perfor- }\end{array}$ & High risk \\
$\begin{array}{l}\text { mance bias) } \\
\text { All outcomes }\end{array}$ & $\begin{array}{l}\text { Quote: "It was not possible to blind either participants or study staff on the na- } \\
\text { ture of the } 2 \text { foods, because RUCF was in paste form and was packed in 250-g } \\
\text { plastic jars, whereas UNIMIX was in flour form and was packed in plastic bags" }\end{array}$ \\
& Comment: not done
\end{tabular}

$\begin{array}{lll}\text { Blinding of outcome as- } & \text { High risk } & \begin{array}{l}\text { Quote: "It was not possible to blind either participants or study staff on the na- } \\ \text { ture of the } 2 \text { foods, because RUCF was in paste form and was packed in } 250-\mathrm{g}\end{array}\end{array}$
All outcomes plastic jars, whereas UNIMIX was in flour form and was packed in plastic bags"

Quote: "Only the statistician was blinded to study groups during the data analysis"

Comment: not done

\begin{tabular}{lll}
\hline $\begin{array}{l}\text { Incomplete outcome data } \\
\text { (attrition bias) }\end{array}$ & Low risk & Attrition: $R$ RUCF group $=656 / 691 ;$ UNIMIX group $=675 / 692$ \\
All outcomes & Comment: reasons provided for loss to follow-up
\end{tabular}

\begin{tabular}{ll}
\hline $\begin{array}{l}\text { Selective reporting (re- } \\
\text { porting bias) }\end{array}$ & Low risk \\
& $\begin{array}{l}\text { Comment: trial registered as ISRCTN20267635 at ISRCTN. Outcomes de- } \\
\text { scribed in the protocol and the methodology section reported in the results } \\
\text { section }\end{array}$
\end{tabular}

\begin{tabular}{lll}
\hline Other bias $\quad$ Low risk $\quad$ Comment: no other potential sources of bias reported \\
\hline
\end{tabular}

\section{Christian 2015}

Methods Design: an unblinded, community-based cluster-randomised controlled trial.

Unit of randomisation: cluster (sectors)

Participants Location/Setting: the study was conducted in the rural north-western study site, located in 18 Unions of the Gaibandha and one Union of the Rangpur district in Bangladesh.

Sample size: 5536 children (6 months of age)

Dropouts/withdrawals: 872 lost to follow-up

Sex: both male and female children included

Mean age: 6.22 months

Inclusion criteria: families who had consented to be enrolled were visited at home for a baseline enrolment interview in the week the child turned 6 months of age, across all 5 groups.

Exclusion criteria: to ensure full exposure to a year of supplementation, per protocol, consented children who were not met despite repeated visits were excluded from the study once they had reached their 7-month birthday.

Interventions Intervention

Preventive lipid-based nutrient supplements given with complementary foods to infants and young children 6 to 23 months of age for health, nutrition, and developmental outcomes (Review)

Copyright $\odot 2019$ The Cochrane Collaboration. Published by John Wiley \& Sons, Ltd. 
Christian 2015 (Continued)
1. $\mathrm{WSB}++(\mathrm{n}=928)$
2. Chickpea $(n=920)$
3. Rice lentil $(n=901)$
4. Plumpy'Doz $(n=1599)$

Control: no food supplement $(n=1591)$

Children received either of the above along with nutrition counselling in all groups.

$\begin{array}{ll}\text { Outcomes } & \text { Primary outcomes } \\ \text { 1. Length } \\ \text { 2. length-for-age } z \text { score }(L A Z) \\ \text { 3. Stunting }(L A Z<2 S D) \\ \text { 4. Weight-for-length } z \text { score }(W L Z) \\ \text { 5. Wasting }(W L Z<2 S D)\end{array}$

Secondary outcomes: not specified

Timing of outcome assessment: at 18 months of age

Study start date: September 2012
Study end date: May 2014
Funding source: this work was supported by the US Department of Agriculture, National Insti-
tute of Food and Agriculture, under the Food and Nutrition Enhancement Program (Award No.
2010-38418-21732). In-kind support in the form of micronutrient premix for the food supplements was
provided by DSM, Basel, Switzerland, and Plumpy'Doz was provided by Nutriset (Maulany, France). Ad-
ditional support for the study was provided by the Bill \& Melinda Gates Foundation (GH614) and the
Johns Hopkins Sight and Life Global Nutrition Research Institute.

Conflicts of interest: the study authors had no conflict of interests to declare.

Comment: none

\section{Risk of bias}

\begin{tabular}{lll}
\hline Bias & Authors' judgement & Support for judgement \\
\hline $\begin{array}{ll}\text { Random sequence genera- } \\
\text { tion (selection bias) }\end{array}$ & Low risk & $\begin{array}{l}\text { Quote: "A random-number seed was selected by a statistician not involved in } \\
\text { the study, using a random number generator, and a random number between }\end{array}$ \\
& & 0 and 1 drawn from a uniform distribution was assigned to each sector"
\end{tabular}

Comment: adequately done

\begin{tabular}{|c|c|c|}
\hline $\begin{array}{l}\text { Allocation concealment } \\
\text { (selection bias) }\end{array}$ & Unclear risk & Comment: not specified \\
\hline $\begin{array}{l}\text { Blinding of participants } \\
\text { and personnel (perfor- } \\
\text { mance bias) } \\
\text { All outcomes }\end{array}$ & High risk & $\begin{array}{l}\text { Quote: "Our trial was unblinded" } \\
\text { Comment: not done }\end{array}$ \\
\hline $\begin{array}{l}\text { Blinding of outcome as- } \\
\text { sessment (detection bias) } \\
\text { All outcomes }\end{array}$ & High risk & $\begin{array}{l}\text { Quote: "Our trial was unblinded" } \\
\text { Comment: not done }\end{array}$ \\
\hline $\begin{array}{l}\text { Incomplete outcome data } \\
\text { (attrition bias) }\end{array}$ & Low risk & $\begin{array}{l}\text { Attrition: control group }=1312 / 1591 ; \text { Plumpy'Doz group }=1395 / 1599 ; \text { rice } \\
\text { lentil group }=785 / 901 ; \text { chickpea group }=786 / 920 ; \text { WSB++ group }=789 / 928\end{array}$ \\
\hline
\end{tabular}

Preventive lipid-based nutrient supplements given with complementary foods to infants and young children 6 to 23 months of age for 
Christian 2015 (Continued)

All outcomes
Comment: reasons provided for loss to follow-up

$\begin{array}{ll}\begin{array}{l}\text { Selective reporting (re- } \\ \text { porting bias) }\end{array} & \text { Low risk } \\ & \begin{array}{l}\text { Comment: published protocol not found. Outcomes described in the method- } \\ \text { ology section reported in the results section }\end{array}\end{array}$

Other bias Low risk Comment: no other potential sources of bias reported

Dewey 2017

Methods Design: a cluster-randomised effectiveness trial within a community-based health program.

Unit of randomisation: cluster

Participants

Location/Setting: 11 rural unions of the Badarganj and Chirirbandar subdistricts in north west Bangladesh

Sample size: 4011 women at $<20$ weeks of gestation within 64 clusters, each comprising the supervision area of a community health worker

Dropouts/withdrawals: 137 lost to follow-up

Sex: both males and females included

Mean age: children were enrolled at birth and the intervention was initiated at 6 months of age.

\title{
Inclusion criteria:
}

1. gestational age $<20$ weeks

2. No plans to move away during pregnancy or the following 3 years

Exclusion criteria: not specified.

\section{Intervention}

1. Women $(n=1047)$ and children $(n=918)$ both received LNS (LNS-LNS group)

2. Women $(n=930)$ received IFA and children $(n=801)$ received LNS (IFA-LNS group)

3. Women $(n=1052)$ received IFA and children $(n=925)$ received MNP (IFA-MNP group)

Control: women $(n=982)$ received IFA and children $(n=872)$ received no supplements (IFA-control group)

For this review, we used data from IFA-LNS group and IFA-control group.

\section{Outcomes}

Primary outcome: length-for-age z score (LAZ).

\section{Secondary outcomes}

\author{
1. Stunting \\ 2. Wasting \\ 3. Underweight \\ 4. Head circumference \\ 5. Weight gain \\ 6. Length gain \\ 7. Weight-for-age z score (WAZ) \\ 8. Weight-for length z score (WLZ) \\ 9. Mid-upper arm circumference (MUAC)
}

Timing of outcome assessment: at 24 months of age.

Preventive lipid-based nutrient supplements given with complementary foods to infants and young children 6 to 23 months of age for health, nutrition, and developmental outcomes (Review)

Copyright $\odot 2019$ The Cochrane Collaboration. Published by John Wiley \& Sons, Ltd. 
Dewey 2017 (Continued) Notes
Study start date: October 2011

Study end date: August 2012

Funding source: USAID provided funding for the study to the University of California, Davis, through the FANTA project.

Conflicts of interest: none of the study authors reported a conflict of interest related to the study.

Comment: we only used data from the group in which only children received LNS compared to control (IFA-LNS group vs IFA-Control group).

\section{Risk of bias}

\begin{tabular}{|c|c|c|}
\hline Bias & Authors' judgement & Support for judgement \\
\hline $\begin{array}{l}\text { Random sequence genera- } \\
\text { tion (selection bias) }\end{array}$ & Low risk & $\begin{array}{l}\text { Quote: "For the randomization, the study statistician at UCD first stratified all } \\
64 \text { clusters in the } 11 \text { unions by subdistrict and union and then randomly as- } \\
\text { signed each cluster to } 1 \text { of the } 4 \text { arms (each containing } 16 \text { clusters)" } \\
\text { Comment: adequately done }\end{array}$ \\
\hline $\begin{array}{l}\text { Allocation concealment } \\
\text { (selection bias) }\end{array}$ & Unclear risk & Comment: not specified \\
\hline $\begin{array}{l}\text { Blinding of participants } \\
\text { and personnel (perfor- } \\
\text { mance bias) } \\
\text { All outcomes }\end{array}$ & High risk & $\begin{array}{l}\text { Quote: "The trial was a researcher-blind, longitudinal, cluster randomized ef- } \\
\text { fectiveness trial" } \\
\text { Comment: not done }\end{array}$ \\
\hline $\begin{array}{l}\text { Blinding of outcome as- } \\
\text { sessment (detection bias) } \\
\text { All outcomes }\end{array}$ & Low risk & $\begin{array}{l}\text { Quote: "The trial was a researcher-blind, longitudinal, cluster randomized ef- } \\
\text { fectiveness trial" } \\
\text { Comment: adequately done }\end{array}$ \\
\hline
\end{tabular}

Incomplete outcome data Low risk (attrition bias)

Attrition: LNS-LNS group = 17\%; IFA-LNS group $=15 \%$; IFA-MNP group $=16 \%$;

All outcomes IFA-control $=16 \%$

Selective reporting (re- Low risk Comment: trial registered as NCT01715038 at ClinicalTrials.gov
porting bias)

Other bias Low risk Comment: no other potential sources of bias reported

Hess 2015

Methods Design: a placebo-controlled, cluster-randomised trial

Unit of randomisation: cluster

Participants

Location/Setting: 34 communities of the Dandé Health District in southwestern Burkina Faso

Sample size: 2435 eligible children were randomly assigned to 1 of 4 intervention groups from 9-18 months of age.

Dropouts/withdrawals: 594 lost to follow-up

Sex: both male and female children included

Preventive lipid-based nutrient supplements given with complementary foods to infants and young children 6 to 23 months of age for 
Hess 2015 (Continued)

\section{Mean age: 9.4 months}

\section{Inclusion criteria:}

1. Children aged 8.8 to 9.9 months

2. Resided permanently in the area

3. Planned to be available during the study period

\section{Exclusion criteria}

1. $\mathrm{Hb}<50 \mathrm{~g} / \mathrm{L}$;

2. Weight-for-length $<70 \%$ of the median of the National Center for Health Statistics/WHO growth reference

3. Presence of bipedal oedema

4. Other severe illness warranting hospital referral

5. Congenital abnormalities potentially interfering with growth

6. Chronic medical conditions requiring frequent medical attention

7. Known HIV infection of infant or mother

8. History of allergy towards peanuts

9. History of anaphylaxis or serious allergic reaction to any substance requiring emergency medical care 10.Concurrent participation in any other clinical trial

\section{Intervention}

1. SQ LNS without zinc, placebo tablet (LNS-Zn0; $\mathrm{n}=602$ )

2. SQ LNS containing $5 \mathrm{mg}$ zinc, placebo tablet (LNS-Zn5; $\mathrm{n}=613$ )

3. SQ LNS containing $10 \mathrm{mg}$ zinc, placebo tablet (LNS-Zn10; $\mathrm{n}=603$ )

4. SQ LNS without zinc and $5 \mathrm{mg}$ zinc tablet (LNS-TabZn5; $\mathrm{n}=617$ )

Control: no intervention (NIC; $n=785$ )

During weekly morbidity surveillance, oral rehydration salts were provided for reported diarrhoea and antimalarial therapy for confirmed malaria; 785 children in the nonintervention group did not receive SQ LNS, tablets, illness surveillance or treatment.

Outcomes

\section{Primary outcomes}

1. Length

2. Weight

3. Mid-upper arm circumference (MUAC)

4. Head circumference

5. Length-for age $z$ score (LAZ)

6. Weight-for-age $z$ score (WAZ)

7. Weight-for-length z score (WLZ)

8. Diarrhoea

9. Malaria

10.Zinc concentration

\section{Secondary outcomes}
1. Stunting
2. Underweight
3. wasting
4. $\mathrm{Hb}$
5. C-reactive protein

Timing of outcome assessment: at 18 months of age

Preventive lipid-based nutrient supplements given with complementary foods to infants and young children 6 to 23 months of age for health, nutrition, and developmental outcomes (Review)

Copyright $\odot 2019$ The Cochrane Collaboration. Published by John Wiley \& Sons, Ltd. 
Hess 2015 (Continued)

Notes
Study start date: April 2010

Study end date: July 2012

Funding source: the project was funded by a grant from the Bill \& Melinda Gates Foundation to the University of California, Davis. The funder had no role in the design of the study, data collection and analysis, the decision to publish, or in the preparation of the manuscript.

Conflicts of interest: none of the study authors had a conflict of interest to declare.

Comment: none

\section{Risk of bias}

Bias Authors' judgement Support for judgement

Random sequence genera- Low risk tion (selection bias)

Quote: "computer-generated an assignment within strata to participate in the intervention cohort .... The same statistician, who was blinded to the intervention, generated a random allocation sequence at the level of the concession for the enrollment of eligible infants in the IC"

Comment: adequately done

Allocation concealment Unclear risk Comment: not clearly stated
(selection bias)

Blinding of participants High risk and personnel (performance bias)

All outcomes

Quote: "The trial was partially masked, as all participants, field staff and researchers remained blinded to the four intervention groups until data analyses were completed, but were aware which communities were assigned to IC and NIC"

Comment: partially blinded

Blinding of outcome as- Low risk sessment (detection bias)

All outcomes
Quote: "Treatment groups remained masked until all statistical analyses were completed and main conclusions were drawn"

Comment: adequately done

Incomplete outcome data Low risk
(attrition bias)
(attrition bias)

All outcomes
Attrition: LNS-Zn0 group = 489/602; LNS-Zn5 group = 499/613; LNS-Zn10 group $=491 / 603 ;$ LNS-TabZn5 group $=481 / 617 ;$ NIC group $=666 / 785$

Comment: reasons for loss to follow-up mentioned

Selective reporting (re- Low risk Comments: protocol attached as a supplement in the study paper
porting bias)

Other bias Low risk Comment: no other potential sources of bias reported

Huybregts 2012

\begin{tabular}{ll}
\hline Methods & Design: a 2-arm cluster-randomised controlled, pragmatic intervention study. \\
& Unit of randomisation: cluster \\
\hline Participants & Location/Setting: city of Abeche, Chad \\
& Sample size: 1038 children (6 to 36 months of age) \\
& Dropouts/withdrawals: 33 lost to follow-up \\
\hline
\end{tabular}

Preventive lipid-based nutrient supplements given with complementary foods to infants and young children 6 to 23 months of age for 49 health, nutrition, and developmental outcomes (Review)

Copyright $\odot 2019$ The Cochrane Collaboration. Published by John Wiley \& Sons, Ltd. 
Huybregts 2012 (Continued)

Sex: both male and female children included

Mean age: 24 months

\section{Inclusion criteria}

1. Non-wasted (weight-for-height $>80 \%$ of the National Center for Health Statistics reference median and lack of bilateral pitting oedema)

2. Being from a "vulnerable" household

Exclusion criteria: not specified

Interventions

Intervention: $46 \mathrm{~g}$ of RUSF, given daily for 4 months $(\mathrm{n}=613)$

Control: no intervention $(n=458)$

Both arms were included in a general food distribution program providing staple foods.

\section{Outcomes}

\section{Primary outcomes}

1. Malnutrition;

2. Wasting

3. Weight-for-height $\mathrm{z}$ score (WHZ) change over time

\section{Secondary outcomes}

1. Prevalence of stunting at end point defined as height-for-age $z$ score (HAZ)

2. Mean HAZ change over time

3. Mid-upper-arm-circumference change over time

4. Mean $\mathrm{Hb}$ concentration at end point

5. Prevalence of anaemia at end point $(\mathrm{Hb}<110 \mathrm{~g} / \mathrm{L})$

6. Morbidity

Timing of outcome assessment: after 4 months of intervention

Study start date: June 2010

Study end date: September 2010

Funding source: this study was funded by Action Contre la Faim, France. The World Food Programme (WFP) donated the food used to compile the food rations. WFP had no role in the design of the study, its implementation, data analysis and interpretation or preparation of the manuscript.

Conflicts of interest: the study authors declared that no competing interests exist.

Comment: none

\section{Risk of bias}

\section{Bias} Authors' judgement Support for judgement

Random sequence genera- High risk tion (selection bias)

Quote: "random assignment was conducted through an official ceremonial gathering with officials and community members. Fourteen papers with cluster numbers were drawn blindly from a bag. Drawn clusters were alternatively assigned to the intervention group and control group"

Comment: not adequate

Allocation concealment High risk

(selection bias)

Quote: "random assignment was conducted through an official ceremonial gathering with officials and community members. Fourteen papers with cluster numbers were drawn blindly from a bag. Drawn clusters were alternatively assigned to the intervention group and control group"

Preventive lipid-based nutrient supplements given with complementary foods to infants and young children 6 to 23 months of age for 50 health, nutrition, and developmental outcomes (Review)

Copyright (c) 2019 The Cochrane Collaboration. Published by John Wiley \& Sons, Ltd. 


\begin{tabular}{|c|c|c|}
\hline $\begin{array}{l}\text { Blinding of participants } \\
\text { and personnel (perfor- } \\
\text { mance bias) } \\
\text { All outcomes }\end{array}$ & High risk & $\begin{array}{l}\text { Quote: "the study participants were not blinded with respect to the interven- } \\
\text { tion assignment because of the type of supplement (paste) provided to chil- } \\
\text { dren" } \\
\text { Comment: not done }\end{array}$ \\
\hline $\begin{array}{l}\text { Blinding of outcome as- } \\
\text { sessment (detection bias) } \\
\text { All outcomes }\end{array}$ & Low risk & $\begin{array}{l}\text { Quote: "Data collectors were blinded to group assignment when measure- } \\
\text { ments were taken" } \\
\text { Comment: adequately done }\end{array}$ \\
\hline $\begin{array}{l}\text { Incomplete outcome data } \\
\text { (attrition bias) } \\
\text { All outcomes }\end{array}$ & Low risk & $\begin{array}{l}\text { Attrition: control group }=440 / 458 \text {; intervention group }=598 / 613 \\
\text { Comment: reasons provided for loss to follow-up }\end{array}$ \\
\hline $\begin{array}{l}\text { Selective reporting (re- } \\
\text { porting bias) }\end{array}$ & Low risk & Comment: trial protocol provided as a supplement \\
\hline Other bias & Low risk & Comment: no other potential sources of bias reported \\
\hline
\end{tabular}

Iannotti 2014

Mesign: a randomised controlled trial with a parallel design.
Unit of randomisation: individual
Location/Setting: urban slum of Cap Haitien in Haiti
Sample size: 589 healthy, singleton infants (aged 6-11 months) recruited
Dropouts/withdrawals: 169 lost to follow-up
Sex: both male and female children included
Mean age: 7.3 months
Inclusion criteria
1. Infant aged between 6 and 11 months
2. Infant in good health (no fever, congenital health condition, or peanut allergy)
3. Singleton birth
4. Infant not severely malnourished (weight-for-length z score (WLZ) less than -3 SD)
5. Household was not receiving other food aid
6. Residence within the Fort Saint Michel catchment area

Exclusion criteria: not specified

\section{Intervention:}

1. 3 -month LNS $(n=196)$;
2. 6 -month LNS $(n=202)$.

Control: no intervention $(n=191)$

The LNS provided $108 \mathrm{kcal}$ and other nutrients, including vitamin A, vitamin B-12, iron, and zinc at $80 \%$ of the recommended amounts. 
Iannotti 2014 (Continued)

Outcomes

\section{Primary outcomes}

1. length

2. height

3. weight

4. $z$ scores for length for age, weight for age, weight for height

5. BMI

6. Stunting

7. Underweight

8. Wasting

\section{Secondary outcomes}

1. IYCF practices

2. Dietary consumption patterns

3. Outcomes of language and motor development

4. Diarrhoea

5. Respiratory infection

6. Cough

7. Fever

8. Helminthes infection

9. Eye and skin conditions

Study end date: December 2012

Funding source: partners in the trial included the Ministry of Public Health and Population (MSPP) in Haiti and Meds and Food for Kids (MFK).

Conflicts of interest: none of the study authors had a conflict of interest.

Comment: none

\section{Risk of bias}

Bias Authors' judgement Support for judgement

Random sequence genera- Unclear risk tion (selection bias)

Quote: "Random assignment was carried out through an allocation-concealment mechanism whereby sealed paper forms that masked group assignments were drawn from a container by mothers by using a simple random assignment ratio of 1:1:1 for group assignments"

Comment: randomisation procedure not mentioned

Allocation concealment Low risk
(selection bias)

Quote: "Random assignment was carried out through an allocation-concealment mechanism whereby sealed paper forms that masked group assignments were drawn from a container by mothers by using a simple random assignment ratio of 1:1:1 for group assignments"

Comment: adequately done

Blinding of participants High risk Comment: probably not done
and personnel (perfor-
mance bias)

Preventive lipid-based nutrient supplements given with complementary foods to infants and young children 6 to 23 months of age for 52 health, nutrition, and developmental outcomes (Review)

Copyright $\odot 2019$ The Cochrane Collaboration. Published by John Wiley \& Sons, Ltd. 
Iannotti 2014 (Continued)

Blinding of outcome as- High risk Comment: probably not done sessment (detection bias)

All outcomes

$\begin{array}{ll}\begin{array}{l}\text { Incomplete outcome data } \\ \text { (attrition bias) }\end{array} & \text { Low risk } \\ \text { All outcomes } & \text { ANS group }=159 / 202\end{array}$

\begin{tabular}{|c|c|c|}
\hline $\begin{array}{l}\text { Selective reporting (re- } \\
\text { porting bias) }\end{array}$ & Low risk & $\begin{array}{l}\text { Comment: trial registered as NCT01552512 at ClinicalTrials.gov. Outcomes de- } \\
\text { scribed in the methodology section reported in the results section }\end{array}$ \\
\hline
\end{tabular}

Other bias Low risk Comment: no other potential sources of bias reported

Kumwenda 2014

Methods Design: a community-based, controlled, single-blind, parallel-group clinical trial.

Unit of randomisation: individual

Participants Location/Setting: southern Malawi

Sample size: 1932 healthy infants (aged between 5.5 and 6.5 months)

Dropouts/withdrawals: 52 lost to follow-up

Sex: both male and female children included

Mean age: 9.9 months

Inclusion criteria: mother-infant pairs were eligible if:

1. infant was enrolled in the main iLiNS-DOSE trial;

2. infant was aged between 9.0 and 10.0 months of age;

3. mother was breastfeeding the infant on demand;

4. mother and infant would be available for the full study period of 2 weeks.

\title{
Exclusion criteria
}

1. Mother was breastfeeding more than 1 infant

2. Mother or infant or both had a severe illness warranting hospital referral

Interventions

\section{Intervention}

1. $10 \mathrm{~g} \mathrm{LNS} / \mathrm{d}$ containing milk powder $(\mathrm{n}=321)$

2. $20 \mathrm{~g}$ LNS/d containing milk powder $(n=322)$

3. $40 \mathrm{~g}$ LNS/d containing milk powder $(n=322)$

4. $20 \mathrm{~g}$ milk-free $L N S /$ day $(n=323)$

5. $40 \mathrm{~g}$ milk-free $L N S /$ day $(n=324)$

Control: no supplement until 18 months of age $(n=320)$

Outcomes

\section{Primary outcomes}

\author{
1. Weight; \\ 2. Length; \\ 3. Mid-upper-arm circumference (MUAC) \\ 4. Length-for age $z$ score (LA)Z
}

Preventive lipid-based nutrient supplements given with complementary foods to infants and young children 6 to 23 months of age for 
Kumwenda 2014 (Continued)

5. Weight-for-age z score (WAZ)

6. Weight-for-length z score (WLZ)

\section{Secondary outcomes}

1. $\mathrm{Hb}$

2. Malarial parasitaemia

Timing of outcome assessment: at 12 months

Study start date: November 2009
Study end date: May 2012
Funding source: this publication is based on research funded by a grant from the Bill \& Melinda Gates
Foundation to the University of California, Davis.

Conflicts of interest: none of the study authors had a conflict of interest.

Comment: none

\section{Risk of bias}

\begin{tabular}{lll}
\hline Bias & Authors' judgement & Support for judgement \\
\hline $\begin{array}{ll}\text { Random sequence genera- } \\
\text { tion (selection bias) }\end{array}$ & Low risk & $\begin{array}{l}\text { Quote: "We used block randomization and a set of opaque envelopes to assign } \\
\text { participants to the intervention groups. The randomization list and envelopes } \\
\text { were prepared by a study statistician not involved in trial implementation" }\end{array}$ \\
& & Comment: adequately done
\end{tabular}

\begin{tabular}{|c|c|c|}
\hline $\begin{array}{l}\text { Allocation concealment } \\
\text { (selection bias) }\end{array}$ & Low risk & $\begin{array}{l}\text { Quote: "We used block randomization and a set of opaque envelopes to assign } \\
\text { participants to the intervention groups" }\end{array}$ \\
\hline & & Comment: adequately done \\
\hline
\end{tabular}

\begin{tabular}{|c|c|c|}
\hline $\begin{array}{l}\text { Blinding of participants } \\
\text { and personnel (perfor- }\end{array}$ & High risk & $\begin{array}{l}\text { Comment: Caregivers and personnel who delivered the intervention were } \\
\text { aware whether or not they were receiving LNS. }\end{array}$ \\
\hline
\end{tabular}
mance bias)

All outcomes

Blinding of outcome as- $\quad$ Low risk
sessment (detection bias)

All outcomes

Quote: "and the code was not disclosed to the researchers or to those assessing the outcomes until all data had been entered and verified in a database"

Comment: adequately done

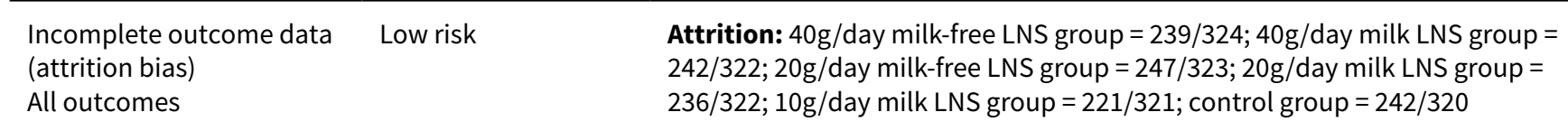

\begin{tabular}{lll}
\hline $\begin{array}{l}\text { Selective reporting (re- } \\
\text { porting bias) }\end{array}$ & Low risk & Comment: trial protocol available on iLiNS Project website \\
\hline Other bias & Low risk & Comment: no other potential sources of bias reported \\
\hline
\end{tabular}

Luby 2018

Methods

Design: cluster-randomised trial

Preventive lipid-based nutrient supplements given with complementary foods to infants and young children 6 to 23 months of age for 
Luby 2018 (Continued)

Unit of randomisation: cluster

Participants

Location/Setting: rural Bangladesh

Sample size: 5551 households

Dropouts/withdrawals: 369 lost to follow-up

Sex: both male and female children included

Mean age: children were enrolled in-utero

\section{Inclusion criteria}

1. The in utero children of enrolled pregnant women (index children) were eligible for inclusion if their mother was planning to live in the study village for the next 2 years, regardless of where she gave birth.

2. Only one pregnant woman was enrolled per compound, but if she gave birth to twins, both children were enrolled.

3. Children who were younger than 3 years at enrolment and lived in the compound were included in diarrhoea measurements.

\section{Exclusion criteria}

1. None specified.

Intervention: pregnant women were grouped into geographic clusters, which were randomly assigned to:

1. chlorinated drinking water $(n=698$ women);

2. upgraded sanitation ( $n=696$ women);

3. handwashing promotion ( $n=688$ women);

4. combined water, sanitation and handwashing (WSH) ( $n=702$ women);

5. a child nutrition intervention, including lipid-based nutrient supplements $(n=699)$;

6. combined WSH plus nutrition $(n=686)$.

Control: no intervention ( $\mathrm{n}=1382$ women)

For this review; we only included outcomes from the child nutrition intervention group and the control group. The nutrition intervention targeted index children. When the index children were between 6 and 24 months of age, promoters provided a regular supply of LNS (provided by Nutriset, Malaunay, France). Promoters instructed caregivers to feed a $10 \mathrm{~g}$ sachet to the index child twice daily, which would provide $118 \mathrm{Kcal}, 9.6 \mathrm{~g}$ of fat, $2.6 \mathrm{~g}$ of protein, 12 vitamins and 10 minerals.

Outcomes

\section{Primary outcomes}

1. Mothers' reports of diarrhoea in the past 7 days among children $<36$ months of age at enrolment

2. Length-for-age $z$ score (LAZ) among children born to the enrolled pregnant women

Secondary outcomes: child developmental outcomes

Timing of outcome assessment: at 1- and 2-year follow-up

Notes

Study start date: May 2012

Study end date: July 2013

Funding source: all study authors declared the receipt of grants and non-financial support from the Bill \& Melinda Gates Foundation during the conduct of this study.

Conflicts of interest: the study authors declared no further competing interests.

Comment: none

Preventive lipid-based nutrient supplements given with complementary foods to infants and young children 6 to 23 months of age for 
Luby 2018 (Continued)

\section{Risk of bias}

\begin{tabular}{|c|c|c|}
\hline Bias & Authors' judgement & Support for judgement \\
\hline $\begin{array}{l}\text { Random sequence genera- } \\
\text { tion (selection bias) }\end{array}$ & Low risk & $\begin{array}{l}\text { Quote: "The randomisation was geographically pair-matched in blocks of } 8 \\
\text { clusters" } \\
\text { Comment: adequately done }\end{array}$ \\
\hline $\begin{array}{l}\text { Allocation concealment } \\
\text { (selection bias) }\end{array}$ & Unclear risk & Comment: not specified \\
\hline $\begin{array}{l}\text { Blinding of participants } \\
\text { and personnel (perfor- } \\
\text { mance bias) } \\
\text { All outcomes }\end{array}$ & High risk & Comment: not done due to the nature of intervention \\
\hline $\begin{array}{l}\text { Incomplete outcome data } \\
\text { (attrition bias) } \\
\text { All outcomes }\end{array}$ & Low risk & $\begin{array}{l}\text { Comment: } \text { nutrition intervention group }=574 / 699(17.88 \%) ; \text { control group }= \\
1138 / 1382(17.65 \%)\end{array}$ \\
\hline $\begin{array}{l}\text { Selective reporting (re- } \\
\text { porting bias) }\end{array}$ & Low risk & Comment: trial registered as NCT01590095 at ClinicalTrials.gov \\
\hline Other bias & Low risk & Comment: no other potential sources of bias reported \\
\hline
\end{tabular}

\section{Mangani 2015}

Methods Design: a community-based randomised trial.

Unit of randomisation: individual

Participants

Location/Setting: 2 rural, health facility catchment areas, Lungwena and Malindi in Mangochi district, southern Malawi.

Sample size: 840, 6-month-old healthy infants residing in the study area

Dropouts/withdrawals: 93 lost to follow-up

Sex: both male and female children included

Mean age: 6.02 months

\section{Inclusion criteria}

1. Aged 5.5 to 6.5 months

2. Residence in the study area

3. Informed consent from at least 1 authorised guardian

\section{Exclusion criteria}

1. Weight-for-length $<80 \%$ of the WHO reference median

2. Presence of oedema

Preventive lipid-based nutrient supplements given with complementary foods to infants and young children 6 to 23 months of age for 
Mangani 2015 (Continued)

3. Severe illness warranting hospitalisation on the enrolment day

4. History of peanut allergy

5. Concurrent participation in another clinical trial

6. Any symptoms of food intolerance within 30 minutes of ingesting a $5 \mathrm{~g}$ test dose of LNS (either milk or soy based) used in the trial

Interventions

\section{Intervention}

1. Milk-LNS $(n=212)$

2. Soy-LNS $(n=210)$

3. Corn-soy blend (CBS; $n=209$

Control: no intervention $(n=209)$

The supplements provided micronutrients and approximately 280 kcal (energy) per day.

$\begin{array}{ll}\text { Outcomes } & \text { Primary outcomes } \\ \text { 1. Severe stunting } \\ \text { 2. Very severe stunting } \\ \text { 3. Moderate-to-severe stunting } \\ \text { Secondary outcomes } \\ \text { 1. Change in length-for-age z score (LAZ) } \\ \text { 2. Changes in weight } \\ \text { 3. Underweight } \\ \text { 4. Wasting } \\ \text { 5. Developmental outcomes }\end{array}$

Timing of outcome assessment: at 18 months

Study end date: May 2009

Funding source: the research project was supported by the Academy of Finland (grant numbers $200720,108873,111685$ and 109796), the Foundation for Pediatric Research in Finland, the Medical Research Fund of Tampere University Hospital and the American people though the support of the Office of Health, Infectious Disease and Nutrition, Bureau for Global Health, USAID, under terms of Cooperative Agreement No. GHN-A-00-08-00001-00, through the FANTA-2 Project, and operated by the Academy for Educational Development (AED).

Conflicts of interest: two study authors received personal stipends from the Nestle Foundation. All other study authors had no conflicts of interest.

Comment: none

\section{Risk of bias}

Bias Authors' judgement Support for judgement

Random sequence genera- Low risk tion (selection bias)
Quote: "Blocked randomisation, with each block containing 16 allocations evenly distributed for the four groups, was used to assign participants to intervention groups"

Comment: adequately done
Quote: "A set of identical-appearing opaque envelopes from one randomisation block was shuffled and a guardian was requested to choose one envelope. 
The envelope contained an identification number and the allocation to one of the four interventions"

Comment: adequately done

\begin{tabular}{lll}
\hline $\begin{array}{l}\text { Blinding of participants } \\
\text { and personnel (perfor- }\end{array}$ & High risk & $\begin{array}{l}\text { Quote: "The main possible causes of bias in our study design were the insuffi- } \\
\text { cient blinding of the field workers to the group allocation" }\end{array}$
\end{tabular}
mance bias)

All outcomes

Comment: not done

\section{Blinding of outcome as- Low risk} sessment (detection bias)

All outcomes
Quote: "The randomisation list and envelopes were made by an individual not involved in trial implementation, and the code was not disclosed to the researchers or to those assessing the outcomes until all data had been entered and verified in a database"

Comment: adequately done

\begin{tabular}{|c|c|c|}
\hline $\begin{array}{l}\text { Incomplete outcome data } \\
\text { (attrition bias) }\end{array}$ & Low risk & $\begin{array}{l}\text { Attrition: control group }=185 / 209 ; \text { milk LNS group }=191 / 212 ; \text { soy LNS group }= \\
188 / 210 ; \text { CSB group }=183 / 209\end{array}$ \\
\hline
\end{tabular}

All outcomes

Comment: mentioned reasons for loss to follow-up

\begin{tabular}{|c|c|c|}
\hline $\begin{array}{l}\text { Selective reporting (re- } \\
\text { porting bias) }\end{array}$ & Low risk & $\begin{array}{l}\text { Comment: key details of the protocol were published at the clinical trial reg- } \\
\text { istry of the National Library of Medicine }\end{array}$ \\
\hline
\end{tabular}

Other bias Low risk Comment: no other potential sources of bias reported

Matias 2017

\begin{tabular}{ll}
\hline Methods & Design: a 2-arm, parallel-group, randomised controlled trial \\
& Unit of randomisation: individual \\
\hline Participants & $\begin{array}{l}\text { Location/Setting: conducted in } 5 \text { rural districts in the Province of Ambo in the Department of Huánu- } \\
\text { co, Peru }\end{array}$
\end{tabular}

Sample size: 422 (6-month-old) children from 19 health centres located in the 5 study districts

Dropouts/withdrawals: 61 lost to follow-up

Sex: both male and female children included

Mean age: 6.1 months

Inclusion criteria: not specified

\section{Exclusion criteria}

1. Chronic, congenital or severe disease that affects growth and metabolism;

2. Not a singleton

3. Low birth weight

4. Not a permanent resident in the study area

Interventions

Children were screened at 5 months of age, but enrolled when they turned 6 months old at the $19 \mathrm{HC}$ located in the 4 study districts and randomly selected to receive either intervention or control.

Intervention: LNS (20 g) containing macronutrients and 19 micronutrients ( $\mathrm{n}=199$ children)

Control: MNP (also known as Sprinkles ${ }^{\mathrm{TM}}$ ), containing 5 micronutrients ( $\mathrm{n}=223$ children)

Preventive lipid-based nutrient supplements given with complementary foods to infants and young children 6 to 23 months of age for 
Matias 2017 (Continued)

Outcomes

\section{Primary outcomes}

1. $\mathrm{Hb}$

2. Anaemia

\section{Secondary outcomes}

1. Gross motor development

2. Language development

3. Cognitive development

Timing of outcome assessment: at 12 months

Study start date: July 2013
Study end date: December 2015
Funding source: this study was funded by the UBS Optimus Foundation (grant \#02) and the Action
Against Hunger (ACH; from its initials in Spanish) Foundation. The LNS (Nutributter ${ }^{\circledR}$ ) were donated by
Nutriset (Malaunay, France). UBS Optimus Foundation and Nutriset had no role in the design, analysis
or writing of the report.

Conflicts of interest: none declared

Comment: none

\section{Risk of bias}

Bias Authors' judgement Support for judgement

Random sequence genera- High risk tion (selection bias)
Quote: "Both supplements were distributed by the MOH HC staff according to the individual-level randomisation plan developed by $\mathrm{ACH}$ Peru following a standard protocol"

Quote: "Due to unexpected problems in the process of customs clearance of the LNS product, the supplement was unavailable for a period of two months (September and October 2014). During that time, the probability of being enrolled in each group differed, but those already enrolled in the LNS group continued receiving their monthly LNS supply"

Comment: not adequate

\begin{tabular}{lll}
\hline $\begin{array}{l}\text { Allocation concealment } \\
\text { (selection bias) }\end{array}$ & High risk & Comment: not specified; probably not done \\
\hline $\begin{array}{l}\text { Blinding of participants } \\
\text { and personnel (perfor- } \\
\text { mance bias) }\end{array}$ & High risk & Comment: not done \\
All outcomes & & \\
\end{tabular}

Blinding of outcome as- $\quad$ High risk $\quad$ Comment: not done
sessment (detection bias)

\begin{tabular}{lll}
\hline $\begin{array}{l}\text { Incomplete outcome data } \\
\text { (attrition bias) } \\
\text { All outcomes }\end{array}$ & Cow risk & Comment: intervention group $=161 / 199(19 \%) ;$ control group $=200 / 223(10 \%)$ \\
\hline $\begin{array}{l}\text { Selective reporting (re- } \\
\text { porting bias) }\end{array}$ & Low risk & $\begin{array}{l}\text { Comment: trial registered as NCT01715038 at ClinicalTrials.gov. Outcomes } \\
\text { specified in the protocol and methodology section reported in the results }\end{array}$ \\
\hline
\end{tabular}

Preventive lipid-based nutrient supplements given with complementary foods to infants and young children 6 to 23 months of age for 
Matias 2017 (Continued)

Other bias High risk

Quote: "Due to unexpected problems in the process of customs clearance of the LNS product, the supplement was unavailable for a period of two months (September and October 2014). During that time, the probability of being enrolled in each group differed, but those already enrolled in the LNS group continued receiving their monthly LNS supply."

Null 2018

$\begin{array}{ll}\text { Methods } & \text { Design: a cluster-randomised trial (Kenya WASH Benefits study) } \\ \text { Unit of randomisation: cluster }\end{array}$

Unit of randomisation: cluster

Participants

Location/Setting: conducted in rural villages in Bungoma, Kakamega, and Vihiga Counties in Kenya's western region

Sample size: 8246 households

Dropouts/withdrawals: 445 lost to follow-up

Sex: both male and female children included

Mean age: children were enrolled in-utero

\section{Inclusion criteria}

1. Villages were eligible for selection into the study if they were:
a. rural;
b. most of the population relied on communal water sources;
c. had unimproved sanitation facilities;
d. no other ongoing water, sanitation, handwashing, or nutrition programmes.

2. Participants were identified through a complete census of eligible villages. Within selected villages, women were eligible to participate if they reported that they:

a. were in their second or third trimester of pregnancy;

b. planned to continue to live at their current residence for the next 2 years;

c. could speak Kiswahili, Luhya, or English well enough to respond to an interviewer administered survey.

Exclusion criteria: not specified

1. Nutrition intervention arm $(n=811)$. Study children between the ages of 6 and 24 months and their age-eligible siblings were provided with $2 \times 10 \mathrm{~g}$ sachets per day of a SQ LNS that could be mixed into the child's food. LNS was manufactured by Nutriset (Malauny, France) and provided 118 kcal per day and 12 essential vitamins and 10 minerals.

Control: active control group $(n=1864)$.

\section{Other study groups}

1. Passive control group $(n=905)$

2. Water intervention group $(n=875)$

3. Sanitation intervention group $(n=856)$

4. Handwashing intervention group $(n=885)$

5. Combined water, sanitation and handwashing group $(n=877)$

6. Combined water, sanitation, handwashing and nutrition group $(n=887)$ 
Null 2018 (Continued)

For this review, we only included outcomes from the nutrition intervention arm and the active control arm

Primary outcomes
1. Caregiver-reported diarrhoea in the past 7
2. Length-for-age $z$ score $(\mathrm{LAZ})$
Secondary and tertiary outcomes
1. Weight-for-length z score (WLZ)
2. Weight-for-age $z$ score (WAZ)
3. Head circumference-for-age $\mathrm{z}$ score $(\mathrm{HCZ})$
4. Prevalence of stunting (LAZ<-2 SD)
5. Severe stunting (LAZ <-3 SD)
6. Wasting (WLZ $<-2 \mathrm{SD})$
7. Underweight (WAZ $<-2 \mathrm{SD})$
8. All-cause mortality

Timing of outcome assessment: at 1-year and 2-year postintervention

Notes

Study start date: November 2012

Study end date: May 2014

Funding source: This research was financially supported in part by Global Development grant OPPGD759 from the Bill \& Melinda Gates Foundation to the University of California, Berkeley, CA, USA, and grant AID-OAA-F-13-00040 from United States Agency for International Development (USAID) to Innovations for Poverty Action. This manuscript was made possible by the generous support of the American people through the USAID.

Conflicts of interest: All study authors received funding for either salary or consulting fees through a grant from the Bill \& Melinda Gates Foundation for this study.

Comment: none

\section{Risk of bias}

Bias Authors' judgement Support for judgement

Random sequence genera- Low risk tion (selection bias)

Quote: "Clusters were randomly allocated to treatment at the University of California, Berkeley using a random number generator with reproducible seed"

Comment: adequately done

\begin{tabular}{ll}
\hline $\begin{array}{l}\text { Allocation concealment } \\
\text { (selection bias) }\end{array}$ & Low risk \\
& Comment: adequately done
\end{tabular}

\begin{tabular}{|c|c|c|}
\hline $\begin{array}{l}\text { Blinding of participants } \\
\text { and personnel (perfor- } \\
\text { mance bias) }\end{array}$ & High risk & $\begin{array}{l}\text { Quote: "Investigators remained blinded to treatment assignments. Masking } \\
\text { participants was not possible" }\end{array}$ \\
\hline All outcomes & & $\begin{array}{l}\text { Comment: blinding of participants was not possible due to the nature of inter- } \\
\text { vention }\end{array}$ \\
\hline
\end{tabular}

Blinding of outcome as-
sessment (detection bias)

Blinding of outcome as-
sessment (detection bias)

All outcomes
Quote: "The health promoters and staff who delivered the interventions were
not involved in data collection, but the data collection team could have in-
ferred treatment status if they saw intervention materials in study communities" 
Null 2018 (Continued)

Comment: not adequately done

\begin{tabular}{lll}
\hline $\begin{array}{l}\text { Incomplete outcome data } \\
\text { (attrition bias) } \\
\text { All outcomes }\end{array}$ & Low risk & $\begin{array}{l}\text { Comment: nutrition intervention group }=695 / 811 \text { (14.3\%); active control } \\
\text { group }=1535 / 1864(17.6 \%)\end{array}$ \\
\hline $\begin{array}{l}\text { Selective reporting (re- } \\
\text { porting bias) }\end{array}$ & Low risk & Comment: trial registered as NCT01704105 at ClincialTrials.gov \\
\hline Other bias & Low risk & Comment: no other potential sources of bias reported \\
\hline
\end{tabular}

Olney 2018

\begin{tabular}{ll}
\hline Design: a longitudinal, cluster-randomised trial (PROCOMIDA, a Food-assisted maternal and child \\
health and nutrition (FA-MCHN)).
\end{tabular}
health and nutrition (FA-MCHN)).

Unit of randomisation: cluster

Location/Setting: Guatemala
Sample size: pregnant women with gestational age between 3 and 7
aged 6 to 24 months ( $=4194)$
Dropouts/withdrawals: 305 lost to follow-up
Sex: both male and female children included
Mean age: not specified
Inclusion criteria: women living in PROCOMIDA communities were
when they became pregnant and could participate in the monthly foo
communication sessions and other program activities from the time
were 24 months of age.
Exclusion criteria: not specified
Intervention
1. Full family ration (FFR) + corn soy blend (CSB) (group 1; $\mathrm{n}=576$ )
2. Reduced family ration (RFR) + CSB (group 2; $\mathrm{n}=575$ )
3. No family ration (NFR) + CSB (group 3; $\mathrm{n}=542$ )
4. FFR + lipid-based nutrient supplement (LNS) (group 4; $\mathrm{n}=550$ )
5. FFR + micronutrient powders (MNP) (group 5; $\mathrm{n}=587$ )

Control: no intervention (group 6; $\mathrm{n}=574$ )

Outcomes

\section{Primary outcomes}

1. Stunting
2. Length-for-age $z$ score (LAZ)

Secondary outcomes: not specified

Timing of outcome assessment: at 24 months of age

Notes Study start date: August 2011

Study end date: May 2015

Preventive lipid-based nutrient supplements given with complementary foods to infants and young children 6 to 23 months of age for 
Funding source: the research took place within the USAID FFP-funded PROCOMIDA program. PRO-

COMIDA was implemented by Mercy Corps in Guatemala.

Conflicts of interest: the study authors declared no conflict of interest.

Comment: none

\begin{tabular}{|c|c|c|}
\hline \multicolumn{3}{|l|}{ Risk of bias } \\
\hline Bias & Authors' judgement & Support for judgement \\
\hline $\begin{array}{l}\text { Random sequence genera- } \\
\text { tion (selection bias) }\end{array}$ & Unclear risk & $\begin{array}{l}\text { Quote: "...eligible health convergence centers were stratified by size and ran- } \\
\text { domly assigned to } 1 \text { of } 6 \text { study groups" }\end{array}$ \\
\hline & & Comment: not specified \\
\hline $\begin{array}{l}\text { Allocation concealment } \\
\text { (selection bias) }\end{array}$ & Unclear risk & Comment: not specified \\
\hline $\begin{array}{l}\text { Blinding of participants } \\
\text { and personnel (perfor- } \\
\text { mance bias) } \\
\text { All outcomes }\end{array}$ & High risk & Comment: probably not done \\
\hline $\begin{array}{l}\text { Blinding of outcome as- } \\
\text { sessment (detection bias) } \\
\text { All outcomes }\end{array}$ & High risk & Comment: probably not done \\
\hline $\begin{array}{l}\text { Incomplete outcome data } \\
\text { (attrition bias) } \\
\text { All outcomes }\end{array}$ & Low risk & $\begin{array}{l}\text { Comment: group } 1=654 / 693 ; \text { group } 2=651 / 695 ; \text { group } 3=632 / 705 ; \text { group } 4= \\
630 / 685 ; \text { group } 5=672 / 718 ; \text { group } 6=650 / 698\end{array}$ \\
\hline $\begin{array}{l}\text { Selective reporting (re- } \\
\text { porting bias) }\end{array}$ & Low risk & Comment: trial registered as NCT01072279 at ClinicalTrials.gov \\
\hline Other bias & Low risk & Comment: no other potential sources of bias reported \\
\hline
\end{tabular}

Phuka 2008

\begin{tabular}{ll}
\hline Methods & Design: a randomised, controlled, single-blind trial. \\
& Unit of randomisation: individual \\
\hline
\end{tabular}

Participants

Location/Setting: Lungwena, a rural Malawian population

Sample size: 182 (6-month-old) infants

Dropouts/withdrawals: 14 lost to follow-up

Sex: both male and female children included

Mean age: 5.9 months

\section{Inclusion criteria}

1. Aged between 5.50 and 6.99 months

2. Residence in the study area

3. Informed consent from at least 1 authorised guardian 
Phuka 2008 (Continued)

\section{Exclusion criteria}

1. Low weight-for-length z score (WLZ) $(<-2.0 \mathrm{SD})$

2. Presence of oedema

3. History of peanut allergy

4. Severe illness warranting hospitalisation on the enrolment day

5. Concurrent participation in another clinical trial

6. Any symptoms of food intolerance 30 minutes after the ingestion of a $6 \mathrm{~g}$ test dose of the peanut-based LNS used as a trial intervention

Interventions
$\begin{aligned} & \text { 1. } 25 \mathrm{~g} / \text { day of micronutrient fortified spread (FS25) for } 1 \text { year }(\mathrm{n}=60) \\ & \text { 2. } 50 \mathrm{~g} / \text { day of micronutrient fortified spread (FS50) for } 1 \text { year }(\mathrm{n}=61)\end{aligned}$

Control: $71 \mathrm{~g} /$ day of micronutrient fortified maize and soy flour (Likuni Phala (LP)) for 1 year $(\mathrm{n}=61$ )

Primary outcome: weight gain
Secondary outcomes
1. Length gain
2. Incidence of severe stunting
3. Underweight
4. Wasting
Timing of outcome assessment: at 12 months

Notes

Study start date: October 2004

Study end date: December 2005

Funding source: the micronutrient mixture used in the production of fortified spread was provided free of charge by Nutriset Incorporate (Malaunay, France)

Conflicts of interest: two of the study authors received a stipend from Nestle Foundation.

Comment: none

\section{Risk of bias}

Bias Authors' judgement Support for judgement

Random sequence genera- Low risk tion (selection bias)

\begin{abstract}
Quote: "For group allocation, guardians picked one from a set of identically appearing opaque envelopes, each containing a paper indicating an identification number and randomly assigned allocation to one of the three interventions. The randomization list and envelopes were made by people not involved in trial implementation and the code was not disclosed to the researchers or those assessing the outcomes until all data had been entered into a database."
\end{abstract}

Comment: adequately done 
Phuka 2008 (Continued)

Comment: adequately done

Blinding of participants High risk Comment: participants unblinded
and personnel (perfor-
mance bias)

\begin{tabular}{ll}
\hline $\begin{array}{l}\text { Blinding of outcome as- } \\
\text { sessment (detection bias) }\end{array}$ & Low risk
\end{tabular}

All outcomes

Comment: adequately done

\begin{tabular}{lll}
\hline $\begin{array}{l}\text { Incomplete outcome data } \\
\text { (attrition bias) } \\
\text { All outcomes }\end{array}$ & Low risk & Attrition: FS50 group = 54/61; FS25 group =57/60; LP group =57/61 \\
\hline $\begin{array}{l}\text { Selective reporting (re- } \\
\text { porting bias) }\end{array}$ & Low risk & $\begin{array}{l}\text { Quote: "Details of the protocol were published at the clinical trial registry of } \\
\text { the National Library of Medicine, Bethesda, Md, USA" }\end{array}$
\end{tabular}

Other bias Low risk Comment: no other potential sources of bias reported

Siega-Riz 2014

\begin{tabular}{ll}
\hline Methods & Design: a cluster-randomised controlled trial. \\
Unit of randomisation: cluster
\end{tabular}

Participants Location/Setting: 3 municipalities (Santa Lucia, Magdalena, and San Antonio) in the south-western part of Intibucá, Honduras, bordering El Salvador

Sample size: 300 children (aged 6-18 months)

Dropouts/withdrawals: 54 lost to follow-up

Sex: both male and female children included

Mean age: not provided (range $=5$ to 18 months of age)

Inclusion criteria: infants and caretakers (mothers/caretakers > 16 years of age) were eligible for the study if:

1. infants were 5-18 months of age at time of recruitment;

2. not participating in a child health brigade that provided vitamin A supplementation;

3. residing within the 3 study municipalities;

4. no plans to move outside of the study region in the next 2 months;

5. no medical conditions.

\section{Exclusion criteria}

1. Infants with congenital anomalies, mental retardation, severe physical handicap, under-nutrition caused by medical conditions, and allergy to peanuts (determined with an allergy reaction test using $5-15 \mathrm{~g}$ of LNS)

2. Infant with a weight for height $z$ score $\leq-2$ SD below the norm; they were referred to a qualified healthcare provider

Interventions

Intervention: participants received a monthly supply of a LNS product (Plumpy'Doz by Nutriset, Malaunay, France) throughout the 12-month study period, providing $247 \mathrm{kcal}(\mathrm{n}=160)$

Preventive lipid-based nutrient supplements given with complementary foods to infants and young children 6 to 23 months of age for 65 health, nutrition, and developmental outcomes (Review)

Copyright (c) 2019 The Cochrane Collaboration. Published by John Wiley \& Sons, Ltd. 


Primary outcomes
Outcomes Micronutrient status (folate, iron, zinc, riboflavin, and vitamin B12 status)
2. $\mathrm{Hb}$

\section{Secondary outcomes}

1. Growth

2. Dietary intake

3. Food insecurity

Timing of outcome assessment: at 12 months

Study start date: March 2009
Study end date: April 2010
Funding source: this work was supported by the Mathile Institute for the Advancement of Human Nu-
trition and The Department of Nutrition Obesity Research Center.

Conflicts of interest: the study authors reported no conflict of interest.

Comment: none

\section{Risk of bias}

\begin{tabular}{lll}
\hline Bias & Authors' judgement & Support for judgement \\
\hline $\begin{array}{ll}\text { Random sequence genera- } \\
\text { tion (selection bias) }\end{array}$ & Unclear risk & $\begin{array}{l}\text { Quote: "One cluster within each pair was then randomised to intervention }(\mathrm{n}= \\
\text { 9) or control group }(\mathrm{n}=9) "\end{array}$
\end{tabular}

Comment: not clearly reported

Allocation concealment High risk
(selection bias)

Quote: "The study was blinded to study group allocation at the data entry level and at the biomarker analysis level. Given the difficulties of working in this rural setting, delivery of the intervention was not blinded for project staff conducting the assessments."

Comment: not done

\begin{tabular}{|c|c|c|}
\hline $\begin{array}{l}\text { Blinding of participants } \\
\text { and personnel (perfor- } \\
\text { mance bias) } \\
\text { All outcomes }\end{array}$ & High risk & $\begin{array}{l}\text { Quote: "The study was blinded to study group allocation at the data entry lev- } \\
\text { el and at the biomarker analysis level. Given the difficulties of working in this } \\
\text { rural setting, delivery of the intervention was not blinded for project staff con- } \\
\text { ducting the assessments." }\end{array}$ \\
\hline
\end{tabular}

Comment: not done

\section{Blinding of outcome as- Low risk} sessment (detection bias) All outcomes
Quote: "The study was blinded to study group allocation at the data entry level and at the biomarker analysis level. Given the difficulties of working in this rural setting, delivery of the intervention was not blinded for project staff conducting the assessments."

Comment: adequately done 
Siega-Riz 2014 (Continued)

Selective reporting (re- Low risk Comment: trial registered as NCT01312987 at ClinicalTrials.gov. Outcomes deporting bias) scribed in the methodology section reported in the results section

Other bias

Low risk

Comment: no other potential sources of bias reported

BMI: body mass index.

FANTA: Food and Nutrition Technical Assistance III Project.

$\mathrm{Hb}$ : haemoglobin

IC: intervention cohort.

ID: identifier

IFA: iron-folic acid.

iLiNS: International Lipid-Based Nutrient Supplements Project.

IYCF: Infant and Young Child Feeding.

LNS: lipid-based nutrient supplements.

MMN: multiple micronutrients.

MNP: micronutrient powders.

$\mathrm{MOH} \mathrm{HC}$ : Ministry of Health Health Centres.

NIC: non-intervention cohort.

RUCF: Ready-to-use complementary food.

RUSF: Ready-to-use supplementary food.

SD: standard deviation.

SQ LNS: small quantity lipid-based nutrient supplements.

UCD: University College Dublin.

UNIMIX: UNICEF's supplementary feeding food.

USAID: US Agency for International Development.

WASH: water, sanitation and hygiene.

WHO: World Health Organization

WSB++: enhanced wheat soy blend.

Characteristics of excluded studies [ordered by study ID]

\begin{tabular}{ll}
\hline Study & Reason for exclusion \\
\hline Ackatia-Armah 2015 & This study included children with moderate acute malnutrition. \\
\hline Adams 2017 & This study assessed combined food security data from three of the included studies. \\
\hline Adams 2018 & $\begin{array}{l}\text { This study is an analysis of 'willingness to pay' from the data of two studies already included in the } \\
\text { review. }\end{array}$ \\
\hline Ahmed 2014 & This study only reported on the development and acceptability of various LNS. \\
\hline Arimond 2017 & This study reported combined results from four studies included in the review \\
\hline Cercamondi 2013 & This study assessed the bioavailability of iron in iron-fortified complementary food. \\
\hline Defourney 2009 & This study is an uncontrolled before-and-after study. \\
\hline Flax 2010 & This study included underweight children. \\
\hline Flax 2013 & This study included HIV-exposed children. \\
\hline Heidkamp 2012 & This study included HIV-exposed children. \\
\hline Isanaka 2009 & This study included children up to 60 months of age. \\
\hline \hline
\end{tabular}

Preventive lipid-based nutrient supplements given with complementary foods to infants and young children 6 to 23 months of age for

Copyright (c) 2019 The Cochrane Collaboration. Published by John Wiley \& Sons, Ltd. 


\begin{tabular}{|c|c|}
\hline Study & Reason for exclusion \\
\hline luel-Brockdorf 2015 & This study included children with moderate acute malnutrition. \\
\hline Kuusipalo 2006 & This study included underweight children. \\
\hline LaGrone 2012 & This study included children with moderate acute malnutrition. \\
\hline Langendorf 2014 & The comparison of interest in this study were not randomly allocated. \\
\hline Maleta 2004 & This study included underweight and stunted children. \\
\hline Maryam 2015 & This study only reported the impact of LNS on vitamin A and zinc levels. \\
\hline Muslihah 2016 & The study design was not appropriate. \\
\hline Rantesalu 2017 & This study reported the impact of LNS on insulin-like growth factor-1 level (IGF-1) only. \\
\hline Schlossman 2017 & This study included mildly or moderately malnourished children. \\
\hline Style 2017 & This study is a before-and-after study without a control group. \\
\hline Thakwalakwa 2010 & This study included moderately underweight children. \\
\hline Thakwalakwa 2012 & This study included underweight children. \\
\hline Thakwalakwa 2015 & This study included moderately underweight children. \\
\hline Unger 2017 & In this study the participants are sick children. \\
\hline Vargas-Vásquez 2015 & This study is an uncontrolled before-and-after study. \\
\hline
\end{tabular}

LNS: lipid-based nutrient supplements.

Characteristics of ongoing studies [ordered by study ID]

\section{Borg 2017}

Trial name or title Public title: Efficacy of a multiple micronutrient-fortified lipid-based nutrient supplement for children under two in Cambodia

Scientific title: Efficacy of a locally-produced multiple micronutrient-fortified lipid-based nutrient supplement (LNS) for children under two years in Cambodia

Methods Design: cluster-randomised, incomplete block, $4 \times 4$ cross-over design with no blinding. Allocation ratio $=1: 1$

Unit of randomisation: cluster

Duration: 6 months

Objective: to establish the superiority of the novel RUSF, using CSB ++ and Sprinkles as active comparators and the unimproved diet as a control.

Participants

Location/setting: 4 sites in a community setting in peri-urban Phnom Penh in Combodia

\section{Prospective sample size: 540}

Sex: both male and female 
Borg 2017 (Continued)

\section{Age range: 6 to 11 months}

\section{Inclusion criteria}

1. Normally nourished or moderately malnourished children

2. Aged 9-23 months

3. In good health for the past 3 days

4. Eating solids for at least 3 months

Only caregivers who have no medical complications or illness will be eligible, in order to avoid any associated appetite loss and to refer for treatment.

\section{Exclusion criteria}

1. Children who have been using Sprinkles, CSB ++ or similar supplementary foods or supplements

2. Children with known food intolerances

Any caregivers or children who become ill during the trial will be excluded and referred for treatment.

Interventions

\section{Intervention}

1. LNS, eaten over 6 months, age $6-12$ months to age $11-17$ months

2. CSB ++ porridge, eaten over 6 months, age 6-12 months to age 11-17 months

3. Sprinkles, eaten over 6 months, age $6-12$ months to age $11-17$ months

Control: no intervention
Primary outcomes: anthropometric status (height-for-age (HAZ), weight-for-height (WHZ) and weight-for-age (WAZ), calculated through monthly weight and height measurements

Starting date $\quad$ February 2016

Current status: active, not recruiting

Contact information

Principal investigator 1: Bindi Borg

Address: School of Public Health, Faculty of Medicine, University of Sydney, Sydney, Australia

Email: bindi_borg@yahoo.com.au

Principal investigator 2: Pascal Marino

Address: European Union Delegation in Cameroon, Yaoundé, Cameroon

Email: not provided

\section{Notes}

\section{Trial registration number: NCT02257762}

Funding source: This work is supported by UNICEF Cambodia; Department of Fisheries Post-Harvest Technologies and Quality Control (DFPTQ), Fisheries Administration, Ministry of Agriculture, Forestry and Fisheries, Cambodia; and IRD France.

Conflicts of interest: none declared 
Scientific title: A cluster-randomised, controlled trial of nutritional supplementation and promotion of responsive parenting in Madagascar: the MAHAY

Design: multi-arm, randomised controlled trial
Unit of randomisation: cluster
Duration: one year
$\begin{aligned} & \text { Objective: to test the effects and cost-effectiveness of combined interventions to address chronic } \\ & \text { malnutrition and poor child development. }\end{aligned}$

\section{Participants}

\section{Location/setting: Madagascar}

Prospective sample size: 25 communities in each arm ( $n=1250$ pregnant women, $n=1250$ children aged $0-6$ months, $n=1250$ children aged $6-18$ months)

Sex: both male and female children

Age range: birth to 18 months

Inclusion criteria: all pregnant women and women with age-eligible children living in the catchment area of a project site are eligible to participate in the standard growth monitoring and nutritional education that occurs in a group setting in a community centre in all sites.

Exclusion criteria: severe malnutrition (will be referred for treatment)

1. (T0) existing program with monthly growth monitoring and nutritional/hygiene education

2. (T1) T0 + home visits for intensive nutrition counselling within a behavior change framework

3. (T2) T1 + lipid-based supplementation (LNS) for children 6-18 months old

4. (T3) T2 + LNS supplementation of pregnant/lactating women

5. (T4) T1 + intensive home visiting program to support child development

There are anticipated to be 25 communities in each arm ( $n=1250$ pregnant women, $n=1250$ children 0-6 months old, and $n=1250$ children $6-18$ months old)

\section{Outcomes}

\section{Primary outcomes}

1. Height-for-age and stunting

2. Measures of child development including language, cognitive development

\section{Secondary outcomes}

1. Wasting

2. Being underweight

3. Iron deficiency and anaemia

4. Intermediate indicators of nutrition and development pathway outcomes including
a. Dietary diversity
b. Household food security
c. Maternal knowledge of child care and feeding practices
d. Home stimulation practices

Starting date July 2014

Current status: no longer recruiting 
Address: School of Public Health, University of California, Berkeley, 50 University Hall, MC 7360, Berkeley, CA 94720-7360, USA

Email: fernald@berkeley.edu

Notes

Trial registration number: ISRCTN14393738

Funding source: Strategic Impact Evaluation Fund; The World Bank

Conflicts of interest: none declared

Huybregts 2017

Trial name or title

Public title: The effect of integrated prevention and treatment on child malnutrition and health in Burkina Faso, a cluster-randomised intervention (PROMIS-BF)

Scientific title: The effect of integrated prevention and treatment on child malnutrition and health in Burkina Faso: a cluster-randomised intervention study

Methods

Design: a two-arm, cluster-randomised, non-masked, community-based trial

Unit of randomisation: cluster

Duration: 24 months

Objective: to assess the effect of an integrated approach consisting of higher screening coverage and preventive Behavior Change Communication (BCC) + SQ-LNS on both prevention and treatment of child undernutrition.

Participants

Location/setting: Burkina Faso and Mali

Prospective sample size: 2400 participants in Burkina Faso and 2304 participants in Mali

Sex: both male and female children

Age range: children aged 6 to 23.9 months

\section{Inclusion criteria}

1. Child aged from birth to 1.4 months

2. Mother living in the study area since the index child's delivery

3. Singleton infants

\section{Exclusion criteria}

1. Congenital malformations that make anthropometric measurements impossible

2. Mother planning to leave the study area in the coming year

3. Children aged 1.5 months or older at study inclusion

4. $W H Z<-2$ both at enrolment and at the first follow-up

Interventions

Intervention: the PROMIS intervention provides an integrated package that consists of 2 key components.

1. Small group, Behavior Change Communication (BCC) on Essential Nutrition Actions (ENA), Infant and Young Child Feeding (IYCF) and Water, Sanitation and Hygiene (WASH) is provided during monthly well-baby visits for children 0-17 months of age;

2. Caregivers with children $0-17$ months of age that attend the well-baby visit will be provided with a monthly dose of LNS ( $20 \mathrm{~g} /$ day). 
Control: monthly well-baby visits, as prescribed by national policy. This arm is the basic comparison arm. Caregivers are invited to frequent the health centre once a month for well-baby visits. During these visits necessary vaccinations are administered, child growth and nutrition status is evaluated and preventive counselling on child nutrition and health is provided in large groups of caregivers.
Outcomes

Starting date

\section{Primary outcomes}

1. Prevalence of acute child malnutrition (defined by $\mathrm{WHZ}<-2$, MUAC $<125 \mathrm{~mm}$ or bilateral pitting oedema in children aged from birth to 17 months) (time frame: after 24 months of program implementation)

2. Screening coverage of acute child malnutrition (proportion of children monthly screened/total number of eligible children (aged from birth to 17 months)) (time frame: monthly from study inclusion at birth to 17 months of age and at study endline)

3. Incidence of child acute malnutrition (defined by $\mathrm{WHZ}<-2$ or MUAC $<125 \mathrm{~mm}$ ) (time frame: monthly from study inclusion at birth to 17 months of age)

4. Compliance to treatment of acute malnutrition (\% of cases that complete treatment over total admitted) (time frame: monthly from study inclusion at birth to 17 months of age and at study endline)

\section{Secondary outcomes}

1. Prevalence of child stunting (defined by $\mathrm{HAZ}<-2$ in children aged from birth to 17 months) (time frame: after 24 months of program implementation)

2. Mean WHZ score in children (aged from birth to 17 months) (time frame: after 24 months of program implementation)

3. Mean HAZ score in children (aged from birth to 17 months) (time frame: after 24 months of program implementation)

4. Mean MUAC in children (aged from birth to 17 months) (time frame: after 24 months of program implementation)

5. Mean $\mathrm{Hb}$ concentration in children (aged from 3 to 17 months) (time frame: after 24 months of program implementation). Hemocues will be used to measure $\mathrm{Hb}$ concentration

6. Prevalence of child anaemia ( $\mathrm{Hb}$ concentration $<10 \mathrm{~g} / \mathrm{dL}$ ) in children (aged from 3 to 17 months) (time frame: after 24 months of program implementation)

7. Prevalence of severe acute child malnutrition (defined by a $\mathrm{WHZ}<-3$, bilateral pitting oedema or a MUAC < $115 \mathrm{~mm}$ in children aged from birth to 17 months) (time frame: after 24 months of program implementation)

8. Prevalence of severe stunting (defined by a $\mathrm{HAZ}<-3$ in children aged from birth to 17 months) (time frame: after 24 months of program implementation)

9. Caregiver's knowledge and practices related to IYCF, ENA and WASH (time frame: after 24 months of program implementation)

10.Incidence of child stunting (defined by HAZ $<-2$ in children aged from birth to 17 months) (time frame: monthly from inclusion at birth to 17 months of age)

11.Linear growth velocity (HAZ increment/month) (time frame: monthly from inclusion at birth to 17 months of age)

12.Ponderal growth velocity (WHZ increment/month) (time frame: monthly from inclusion at birth to 17 months of age)

13. Weight gain (weight increment/month) (time frame: monthly from inclusion at birth to 17 months of age)

14.MUAC (increment/month) (time frame: monthly from inclusion at birth to 17 months of age)

15.Infant morbidity (acute respiratory infections, fever, malaria (RDT), vomiting, diarrhoea) (time frame: monthly from inclusion at birth to 17 moths of age). Malaria will be tested in case of fever (or recalled fever over last 24 hours) using rapid tests

16. Relapse rate after treatment of MAM/SAM (proportion $\mathrm{WHZ}<-2$, MUAC $<125 \mathrm{~mm}$ or bilateral pitting oedema after discharge from MAM or SAM treatment program over total number of children treated) (time frame: monthly from inclusion at birth to 17 months of age)

Preventive lipid-based nutrient supplements given with complementary foods to infants and young children 6 to 23 months of age for

health, nutrition, and developmental outcomes (Review)

Copyright (c) 2019 The Cochrane Collaboration. Published by John Wiley \& Sons, Ltd. 
Current status: recruitment complete

\begin{tabular}{ll}
\hline Contact information & Principle investigator: Lieven Huybregts \\
& Address: International Food Policy Research Institute \\
& Email: L.Huybregts@cgiar.org \\
\hline Notes & Trial registration number: NCT02323815; NCT02245152 \\
& $\begin{array}{l}\text { Funding source: Global Affairs Canada, award number 52308/5252/0200. Co-funding was kindly } \\
\text { provided by the CGIAR Research Program on Agriculture for Nutrition and Health (A4NH), led by the } \\
\text { International Food Policy Research Institute.). }\end{array}$
\end{tabular}

Conflicts of interest: none declared

\section{ISRCTN94319790}

Trial name or title

Public title: Ready to use supplementary foods (RUSF) to prevent stunting among children under five years in Kurram Agency

Scientific title: A community-based cluster-controlled trial to evaluate the effectiveness of ready to use supplementary foods (RUSF) and proportional contribution of multi-sectoral interventions in the prevention of stunting among children under five years in Kurram Agency, Pakistan

Methods

Design: community-based, cluster-randomised controlled trial

Unit of randomisation: cluster

Duration: from pregnancy until 36 months of age.

Objective: the aim of this study is to evaluate the effectiveness of selected nutrition-specific interventions in improving childhood length-for-age scores.

\section{Participants}

\section{Location/setting: Pakistan \\ Prospective sample size: 7200}

Sex: both male and female children

Age range: pregnant women aged 15-49 years; children aged 6-23 months

\section{Inclusion criteria}

1. Group 1: pregnant women in their first trimester (preferably 1st 2 months of pregnancy) will be invited and those between the ages of $15-49$ years will be recruited

2. Group 2: lactating women within the early days following delivery (preferably within 2 months of delivery) will be invited and recruited

3. Group $3^{*}$ : children between 6-18 months of age will be invited and children aged 6-23 months will be recruited

4. Group $4^{\star}$ : children with ages between $24-48$ months will be invited and children aged $24-59$ month will be recruited

*Preferably these children will be recruited from the households from which pregnant and lactating women were invited.

\section{Exclusion criteria}

1. Children with SAM or MAM (will be referred for treatment)

2. Similar nutrition specific interventions currently being implemented in the selected cluster

Preventive lipid-based nutrient supplements given with complementary foods to infants and young children 6 to 23 months of age for 


\section{Intervention}

1. LNS for pregnant and lactating women (Local name: Maamta). These RUSF are given to pregnant and lactating women in Group 1 and Group 2. Supplementation will start from conception/earliest confirmation of pregnancy, or during the first 6 months of lactation for lactating women. Active ingredients include roasted chickpeas, roasted yellow lentils, roasted peanuts, soybean oil, palm olein oil, hydrogenated vegetable fat as stabiliser, skimmed milk powder, sugar, maltodextrin, vitamins and minerals, emulsifier and antioxidant. The daily ration is a $75 \mathrm{~g}$ sachet. One sachet per day is recommended for maintenance of nutritional status, throughout pregnancy and the first 6 months of lactation. Maamta is manufactured within a quality and food safety management environment in accordance with latest version of recognised international standards and best practices and/or guidelines.

2. LNS for children (Local name: Wawa-mum). These RUSF are given to children aged 6-23 months (Group 3 in the trial). The active ingredients include roasted chickpeas, vegetable oil, dry skimmed milk, sugar, vitamins and minerals, emulsifier and antioxidant. The daily ration is a $50 \mathrm{~g}$ sachet. One sachet per day is recommended. The average duration of the intervention is 18 months under stunting prevention, depending on the age of the child at the time of assistance, starting from 6 months till 23 months age. Wawa-mum is manufactured within a quality and food safety management environment in accordance with latest version of recognised international standards and best practices and/or guidelines.

3. Micronutrient Powder (MNP) for children (Local name: Vita-Mixe). These RUSF are given to children aged 24-59 months (i.e. group 4 of the trial). MNP is homogeneous, stable and dry packaged in a $1 \mathrm{~g}$ sachet. The daily ration is $1 \mathrm{~g}$ sachet. One sachet per alternate day is recommended under stunting prevention (1 sachet for 2 days).

Control: no intervention

\section{Primary outcome measures}

1. Change in LAZ, measured using the height boards (SECA) at each month in the intervention vs control arms, compared to the baseline LAZ score against WHO 2006 growth standards (time frame: baseline and thereafter monthly, till the end of the study (3 years)).

2. Change in WAZ, measured using the digital weighing scales (SECA) at each month in intervention vs control arms, compared to the baseline WAZ score against WHO 2006 growth standards (time frame: baseline and thereafter monthly, till the end of the study (3 years)).

3. Change in WHZ, measured using the digital weighing scales (SECA) and height boards (SECA) at each month in intervention vs control arms, compared to the baseline WHZ score against WHO 2006 growth standards (timeframe: baseline and thereafter monthly, till the end of the study (3 years)).

\section{Secondary outcome measures}

1. Infant and Young Child Feeding (IYCF) indicators, measured using a questionnaire (WHO guideline-based) at baseline and at the end of the study in the intervention vs control arm (time frame: baseline and at the end of the study).

2. Birth weight of live newborns, measured using digital weighing scales (SECA) at 24 hours of birth in the intervention vs control arm (time frame: within 24 hours of delivery).

3. Nutritional intake (energy and protein), measured using 24-hour dietary recall method (FAO guideline-based) at baseline and at the end of the study (time frame: baseline and at the end of the study).

4. Maternal weight gain during pregnancy, measured using digital weighing scales (SECA) at baseline and thereafter monthly, till delivery, in the intervention vs control arm (time frame: baseline, thereafter monthly till delivery).

5. $\mathrm{Hb}$, albumin and micronutrients, like iron, zinc, iodine and vitamins, measured using blood samples at baseline and thereafter yearly, until the end of the study (3rd year end), in a subsample in the intervention vs control arm (time frame: baseline, thereafter yearly till the end of the study (3rd year end)). 
ISRCTN94319790 (Continued)

Starting date

December 2017

Current status: ongoing; no longer recruiting

Contact information Principal investigator: Muhammad Naseem Khan

Address: Institute of Public Health \& Social Sciences, Khyber Medical University, Phase 5, Hayatabad 25000, Peshawar, Pakistan

Email: drnasim@kmu.edu.pk

\section{Notes}

Trial registration number: ISRCTN94319790

Funding source: United Nations World Food Programme (WFP)

Conflicts of interest: not specified

\section{SHINE trial 2015}

Trial name or title

Public title: SHINE sanitation, hygiene, infant nutrition efficacy project (SHINE)

Scientific title: Sanitation, Hygiene Infant Nutrition Efficacy Project

Methods

Design: 2 × 2 factorial, cluster-randomised, community-based trial

Unit of randomisation: cluster

Duration: 18 months

Objective: to assess the impact of sanitation, hygiene, infant nutrition on infant length and $\mathrm{Hb}$

\section{Participants}

Location/setting: 2 rural districts of Zimbabwe

Prospective sample size: 4800 pregnant women (1200 in each of the 4 trial arms, each of which is comprised of 53 clusters)

Sex: both male and female

Age range: pregnant women and their infants till 18 months of age

\section{Inclusion criteria}

1. Pregnant women residing in the study districts

2. Pregnancy confirmed by a urine pregnancy test

\section{Exclusion criteria}

1. Women residing in the study districts who become pregnant during the enrolment period but do not consent to join the trial

2. Women who reside in urban areas of these two districts

Infants with major non-fatal abnormalities will not be excluded from study procedures, but will be excluded from the final analytic sample if the abnormality is likely to directly affect gut health/ function or stature (e.g. neural tube defects, cerebral palsy, Down syndrome).

Interventions

\section{Intervention}

1. Water, Sanitation, and Hygiene (WASH) intervention $(n=1200)$ : a package of interventions to improve household sanitation and hygiene 
2. Infant and Young Child fFeding (IYCF) intervention ( $n=1200)$ : a package of interventions to improve infant and young child feeding and Dietary Supplement

3. Sanitation/Hygiene AND nutrition (WASH + IYCF) $(n=1200)$ : this arm will receive a combination of all standard care interventions, all WASH and all IYCF interventions

Control: standard care $(n=1200)$. The standard care intervention is the blanket intervention as routine.

Primary outcomes
1. LAZ - recumbent length, measured by length board
2. $\mathrm{Hb}$
3. level, measured by Hemocue

Timing of outcome assessment: 18 months of age

\begin{tabular}{ll}
\hline Starting date & November 2012 \\
& Current status: completed \\
\hline Contact information & Principal investigator: Jean H Humphrey, ScD \\
& Address: Johns Hopkins Bloomberg School of Public Health \\
& Email: not specified \\
\hline Notes & Trial registration number: NCT01824940 \\
& Funding source: Johns Hopkins Bloomberg School of Public Health \\
& Conflicts of interest: not specified \\
\hline
\end{tabular}

HAZ: Height-for-age z score

$\mathrm{Hb}$ : haemoglobin

LAZ: Length-for-age z score

LNS: lipid-based nutrient supplement

MAHAY: 'smart' in Malagasy

MAM: Moderate acute malnutrition

MUAC: Mid-upper arm circumference

RDT: Rapid diagnostic tests

RUSF: Ready to use supplementary foods

SAM: Severe acute malnutrition

SQ LNS: Small-quantity lipid-based nutrient supplement

WAZ: Weight-for-age z scores

WHI: World Health Organization

WHZ: Weight-for-height z score

\section{DATA AND ANALYSES}

\section{Comparison 1. LNS versus no intervention}

\begin{tabular}{lllll}
\hline Outcome or subgroup title & No. of studies & $\begin{array}{l}\text { No. of partici- } \\
\text { pants }\end{array}$ & Statistical method & Effect size \\
\hline 1 Moderate stunting & 9 & 13372 & Risk Ratio (Random, 95\% Cl) & $0.93[0.88,0.98]$ \\
\hline 2 Severe stunting & 5 & 6151 & Risk Ratio (Random, 95\% Cl) & $0.85[0.74,0.98]$ \\
\hline \hline
\end{tabular}

Preventive lipid-based nutrient supplements given with complementary foods to infants and young children 6 to 23 months of age for 76 health, nutrition, and developmental outcomes (Review)

Copyright $\odot 2019$ The Cochrane Collaboration. Published by John Wiley \& Sons, Ltd. 


\begin{tabular}{|c|c|c|c|c|}
\hline Outcome or subgroup title & No. of studies & $\begin{array}{l}\text { No. of partici- } \\
\text { pants }\end{array}$ & Statistical method & Effect size \\
\hline $\begin{array}{l}3 \text { Moderate stunting: Sensi- } \\
\text { tivity analysis }\end{array}$ & 7 & & Risk Ratio (Random, 95\% Cl) & $0.92[0.88,0.96]$ \\
\hline $\begin{array}{l}4 \text { Severe stunting: Sensitivity } \\
\text { analysis }\end{array}$ & 4 & & Risk Ratio (Random, 95\% Cl) & $0.84[0.73,0.97]$ \\
\hline 5 Moderate wasting & 8 & 13172 & Risk Ratio (Random, 95\% Cl) & $0.82[0.74,0.91]$ \\
\hline 6 Severe wasting & 3 & 2329 & Risk Ratio (Random, 95\% Cl) & $1.27[0.66,2.46]$ \\
\hline $\begin{array}{l}7 \text { Moderate wasting: Sensitiv- } \\
\text { ity analysis }\end{array}$ & 6 & & Risk Ratio (Random, 95\% Cl) & $0.81[0.73,0.90]$ \\
\hline $\begin{array}{l}8 \text { Severe wasting: Sensitivity } \\
\text { analysis }\end{array}$ & 2 & & Risk Ratio (Random, 95\% Cl) & $1.24[0.61,2.51]$ \\
\hline 9 Moderate underweight & 8 & 13073 & Risk Ratio (Random, 95\% Cl) & $0.85[0.80,0.91]$ \\
\hline 10 Severe underweight & 2 & 1729 & Risk Ratio (Random, 95\% Cl) & $0.78[0.54,1.13]$ \\
\hline $\begin{array}{l}11 \text { Moderate underweight: } \\
\text { Sensitivity analysis }\end{array}$ & 6 & & Risk Ratio (Random, 95\% Cl) & $0.85[0.80,0.90]$ \\
\hline $\begin{array}{l}12 \text { Severe underweight: Sen- } \\
\text { sitivity analysis }\end{array}$ & 1 & & Risk Ratio (Random, 95\% Cl) & $0.79[0.54,1.16]$ \\
\hline 13 Anaemia & 5 & 2332 & Risk Ratio (Random, 95\% Cl) & $0.79[0.69,0.90]$ \\
\hline 14 Adverse effects & 3 & 3382 & Risk Ratio (Random, 95\% Cl) & $0.86[0.74,1.01]$ \\
\hline $\begin{array}{l}15 \text { Adverse effects: Sensitivity } \\
\text { analysis }\end{array}$ & 1 & 1932 & Risk Ratio (Random, 95\% Cl) & $0.76[0.60,0.95]$ \\
\hline $\begin{array}{l}16 \text { Mid-upper arm circumfer- } \\
\text { ence (MUAC) }\end{array}$ & 6 & 8187 & $\begin{array}{l}\text { Std. Mean Difference (Random, } \\
95 \% \mathrm{Cl} \text { ) }\end{array}$ & $0.13[0.05,0.22]$ \\
\hline 17 Serum haemoglobin (g/L) & 4 & 4518 & Mean Difference (Random, 95\% Cl) & $5.78[2.27,9.30]$ \\
\hline 18 Mortality & 3 & 3321 & Risk Ratio (Random, 95\% Cl) & $0.93[0.63,1.37]$ \\
\hline $19 \mathrm{HAZ}$ & 12 & 15795 & $\begin{array}{l}\text { Std. Mean Difference (Random, } \\
95 \% \mathrm{Cl} \text { ) }\end{array}$ & $0.11[0.05,0.16]$ \\
\hline 20 WAZ & 10 & 12188 & $\begin{array}{l}\text { Std. Mean Difference (Random, } \\
95 \% \mathrm{Cl} \text { ) }\end{array}$ & $0.09[0.02,0.16]$ \\
\hline $21 \mathrm{WHZ}$ & 10 & 12894 & $\begin{array}{l}\text { Std. Mean Difference (Random, } \\
95 \% \mathrm{Cl} \text { ) }\end{array}$ & $0.08[0.04,0.13]$ \\
\hline
\end{tabular}


Analysis 1.1. Comparison 1 LNS versus no intervention, Outcome 1 Moderate stunting.

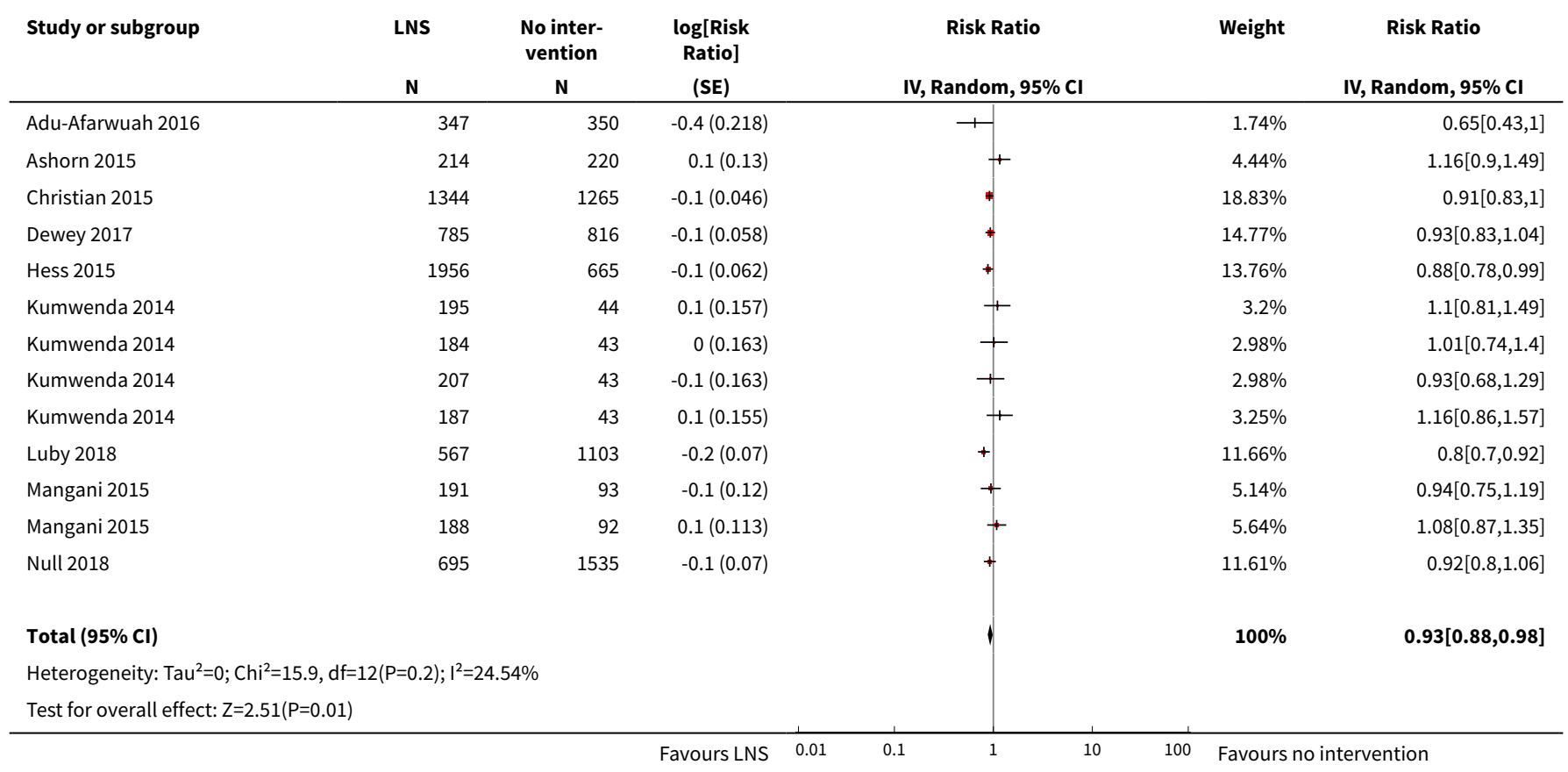

Analysis 1.2. Comparison 1 LNS versus no intervention, Outcome 2 Severe stunting.

\begin{tabular}{|c|c|c|c|c|c|c|}
\hline \multirow[t]{2}{*}{ Study or subgroup } & \multirow{2}{*}{$\begin{array}{l}\text { LNS } \\
\mathrm{N}\end{array}$} & \multirow{2}{*}{$\begin{array}{l}\text { No inter- } \\
\text { vention } \\
\mathbf{N}\end{array}$} & \multirow{2}{*}{$\begin{array}{c}\log [\text { Risk } \\
\text { Ratio] } \\
\text { (SE) }\end{array}$} & \multirow{2}{*}{$\begin{array}{c}\text { Risk Ratio } \\
\text { IV, Random, } 95 \% \mathrm{Cl}\end{array}$} & Weight & \multirow{2}{*}{$\begin{array}{c}\text { Risk Ratio } \\
\text { IV, Random, } 95 \% \mathrm{CI}\end{array}$} \\
\hline & & & & & & \\
\hline Ashorn 2015 & 214 & 220 & $0(0.301)$ & 1 & $5.73 \%$ & $1.03[0.57,1.86]$ \\
\hline Kumwenda 2014 & 254 & 60 & $-0(0.229)$ & $\rightarrow$ & $9.93 \%$ & $0.97[0.62,1.52]$ \\
\hline Kumwenda 2014 & 249 & 60 & $-0.1(0.231)$ & $\rightarrow$ & $9.73 \%$ & $0.92[0.58,1.44]$ \\
\hline Kumwenda 2014 & 262 & 60 & $-0.3(0.247)$ & $\rightarrow$ & $8.56 \%$ & $0.72[0.45,1.17]$ \\
\hline Luby 2018 & 567 & 1103 & $-0.3(0.163)$ & $\rightarrow$ & $19.51 \%$ & $0.74[0.54,1.02]$ \\
\hline Mangani 2015 & 188 & 92 & $0.1(0.247)$ & $\rightarrow$ & $8.52 \%$ & $1.14[0.7,1.84]$ \\
\hline Mangani 2015 & 191 & 93 & $-0.2(0.275)$ & $\rightarrow$ & $6.89 \%$ & $0.78[0.46,1.34]$ \\
\hline Null 2018 & 695 & 1535 & $-0.2(0.152)$ & - & $22.54 \%$ & $0.85[0.63,1.14]$ \\
\hline Total $(95 \% \mathrm{Cl})$ & & & & $\checkmark$ & $100 \%$ & $0.85[0.74,0.98]$ \\
\hline Test for overall effect & & & & & & \\
\hline
\end{tabular}

Analysis 1.3. Comparison 1 LNS versus no intervention, Outcome 3 Moderate stunting: Sensitivity analysis.

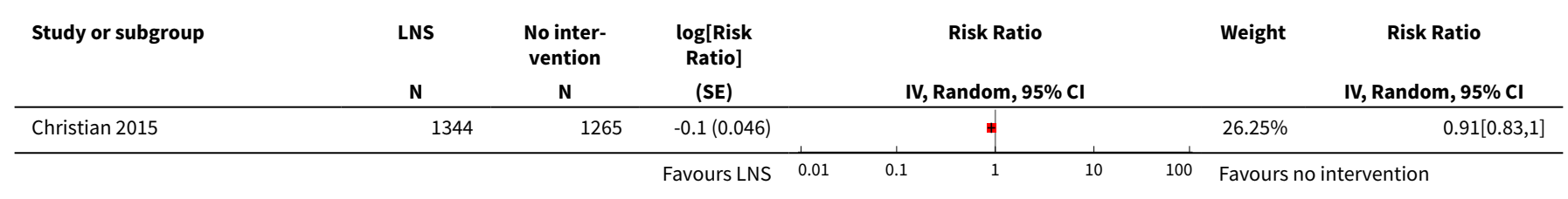




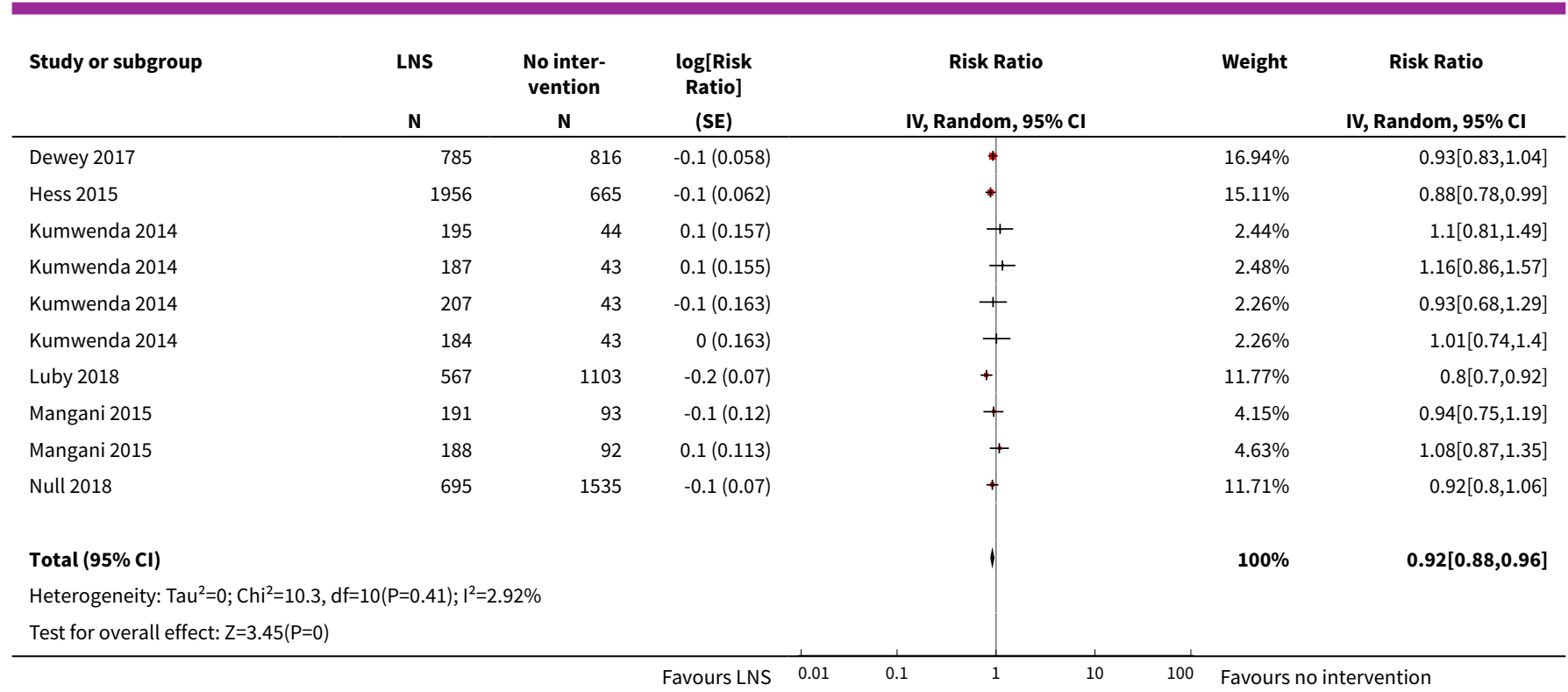

Analysis 1.4. Comparison 1 LNS versus no intervention, Outcome 4 Severe stunting: Sensitivity analysis.

\begin{tabular}{|c|c|c|c|c|c|c|}
\hline Study or subgroup & $\begin{array}{l}\text { LNS } \\
\mathbf{N} \\
\end{array}$ & $\begin{array}{c}\text { No inter- } \\
\text { vention } \\
\mathbf{N} \\
\end{array}$ & $\begin{array}{c}\text { log[Risk } \\
\text { Ratio] } \\
\text { (SE) }\end{array}$ & $\begin{array}{c}\text { Risk Ratio } \\
\text { IV, Random, 95\% CI }\end{array}$ & Weight & $\begin{array}{c}\text { Risk Ratio } \\
\text { IV, Random, } 95 \% \mathrm{CI} \\
\end{array}$ \\
\hline Kumwenda 2014 & 249 & 60 & $-0.1(0.231)$ & $\rightarrow$ & $10.32 \%$ & $0.92[0.58,1.44]$ \\
\hline Kumwenda 2014 & 254 & 60 & $-0(0.229)$ & 1 & $10.53 \%$ & $0.97[0.62,1.52]$ \\
\hline Kumwenda 2014 & 262 & 60 & $-0.3(0.247)$ & $\rightarrow$ & $9.08 \%$ & $0.72[0.45,1.17]$ \\
\hline Kumwenda 2014 & 248 & 60 & $-0.3(0.246)$ & $\rightarrow$ & $9.11 \%$ & $0.76[0.47,1.23]$ \\
\hline Luby 2018 & 567 & 1103 & $-0.3(0.163)$ & $\rightarrow$ & $20.69 \%$ & $0.74[0.54,1.02]$ \\
\hline Mangani 2015 & 188 & 92 & $0.1(0.247)$ & + & $9.04 \%$ & $1.14[0.7,1.84]$ \\
\hline Mangani 2015 & 191 & 93 & $-0.2(0.275)$ & $\rightarrow$ & $7.31 \%$ & $0.78[0.46,1.34]$ \\
\hline Null 2018 & 695 & 1535 & $-0.2(0.152)$ & $\rightarrow$ & $23.91 \%$ & $0.85[0.63,1.14]$ \\
\hline Total $(95 \% \mathrm{Cl})$ & & & & $\checkmark$ & $100 \%$ & $0.84[0.73,0.97]$ \\
\hline \multicolumn{7}{|c|}{ Heterogeneity: $\mathrm{Tau}^{2}=0 ; \mathrm{Chi}^{2}=3.27, \mathrm{df}=7(\mathrm{P}=0.86) ; \mathrm{I}^{2}=0 \%$} \\
\hline \multicolumn{7}{|c|}{ Test for overall effect: $Z=2.37(P=0.02)$} \\
\hline
\end{tabular}

Analysis 1.5. Comparison 1 LNS versus no intervention, Outcome 5 Moderate wasting.

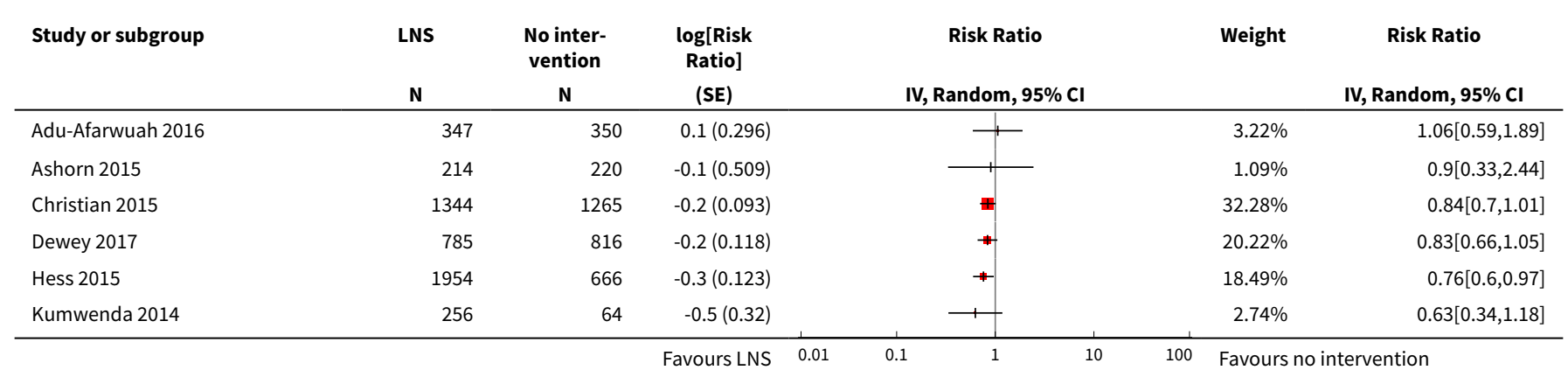




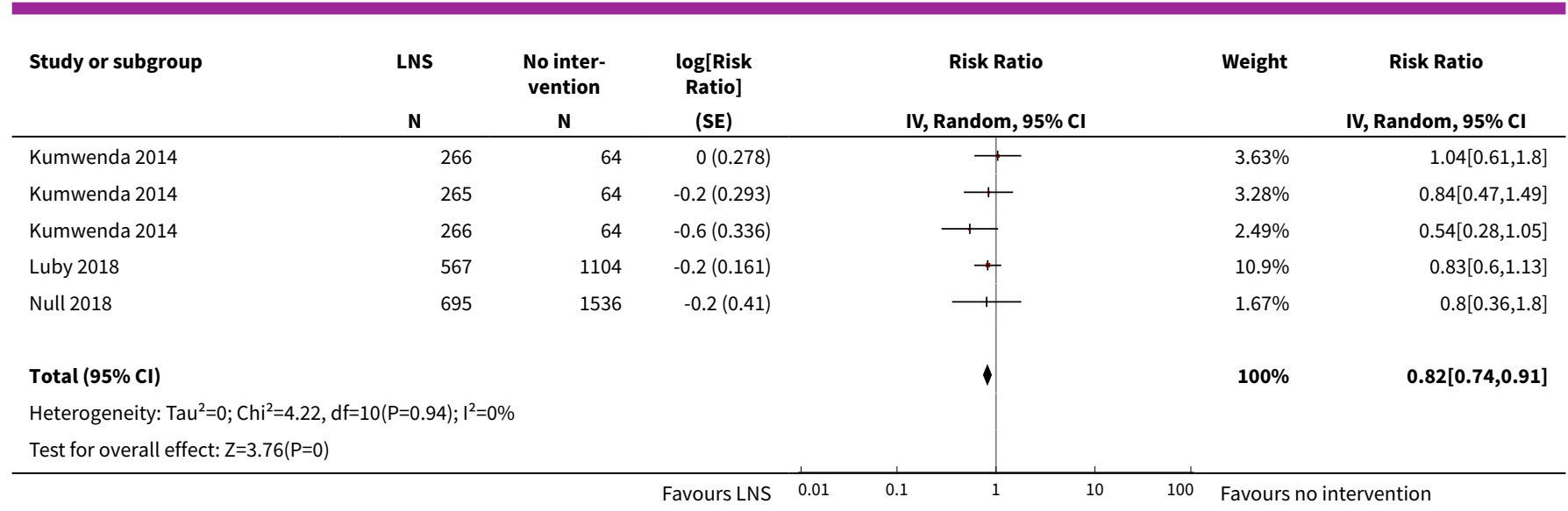

Analysis 1.6. Comparison 1 LNS versus no intervention, Outcome 6 Severe wasting.

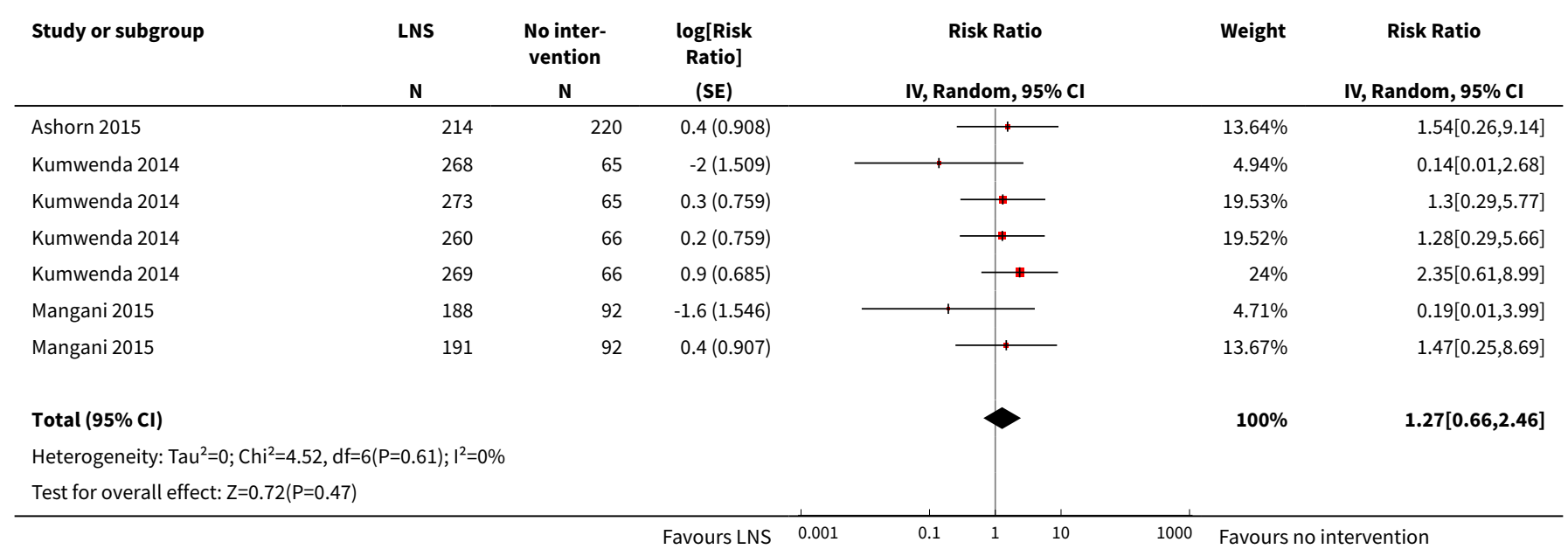

Analysis 1.7. Comparison 1 LNS versus no intervention, Outcome 7 Moderate wasting: Sensitivity analysis.

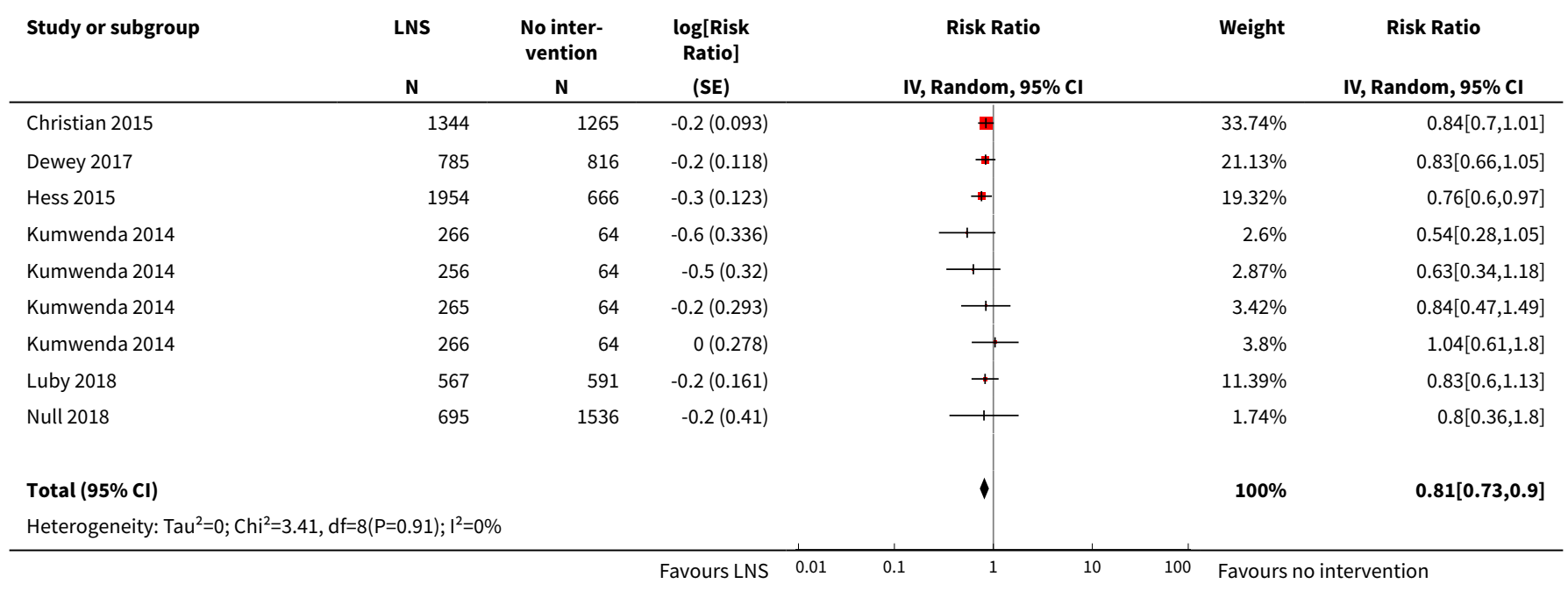




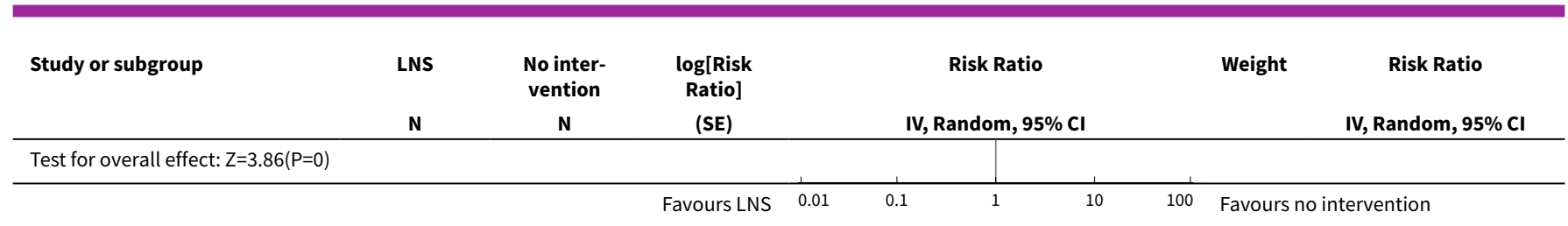

Analysis 1.8. Comparison 1 LNS versus no intervention, Outcome 8 Severe wasting: Sensitivity analysis.

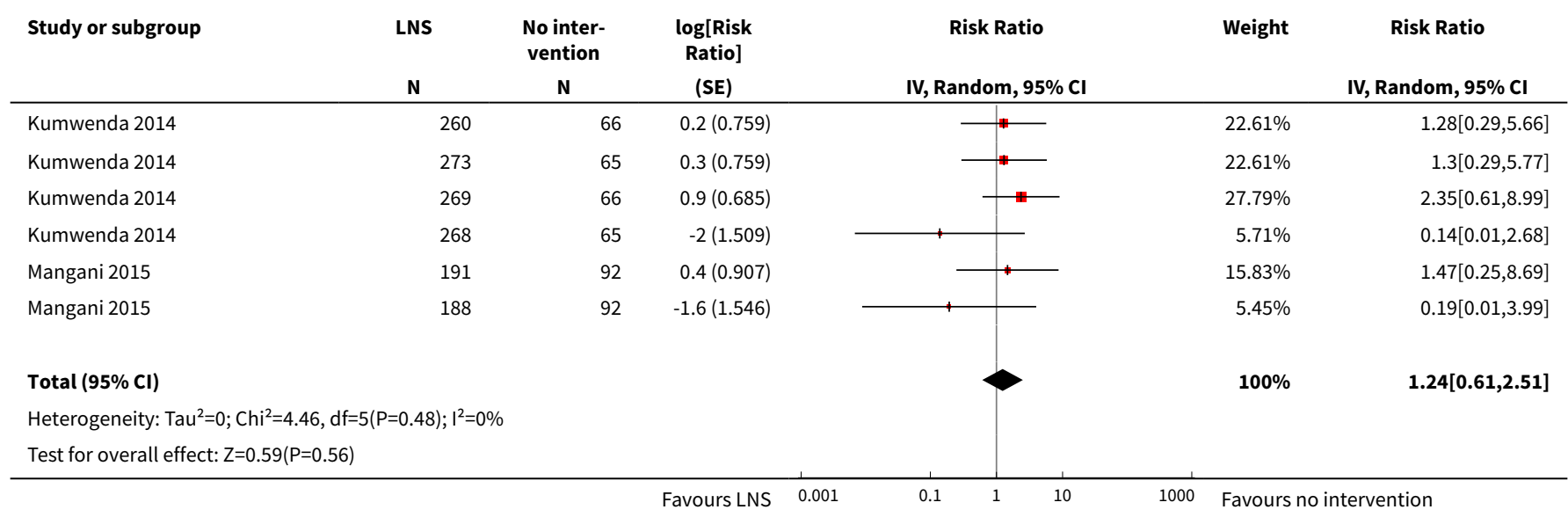

Analysis 1.9. Comparison 1 LNS versus no intervention, Outcome 9 Moderate underweight.

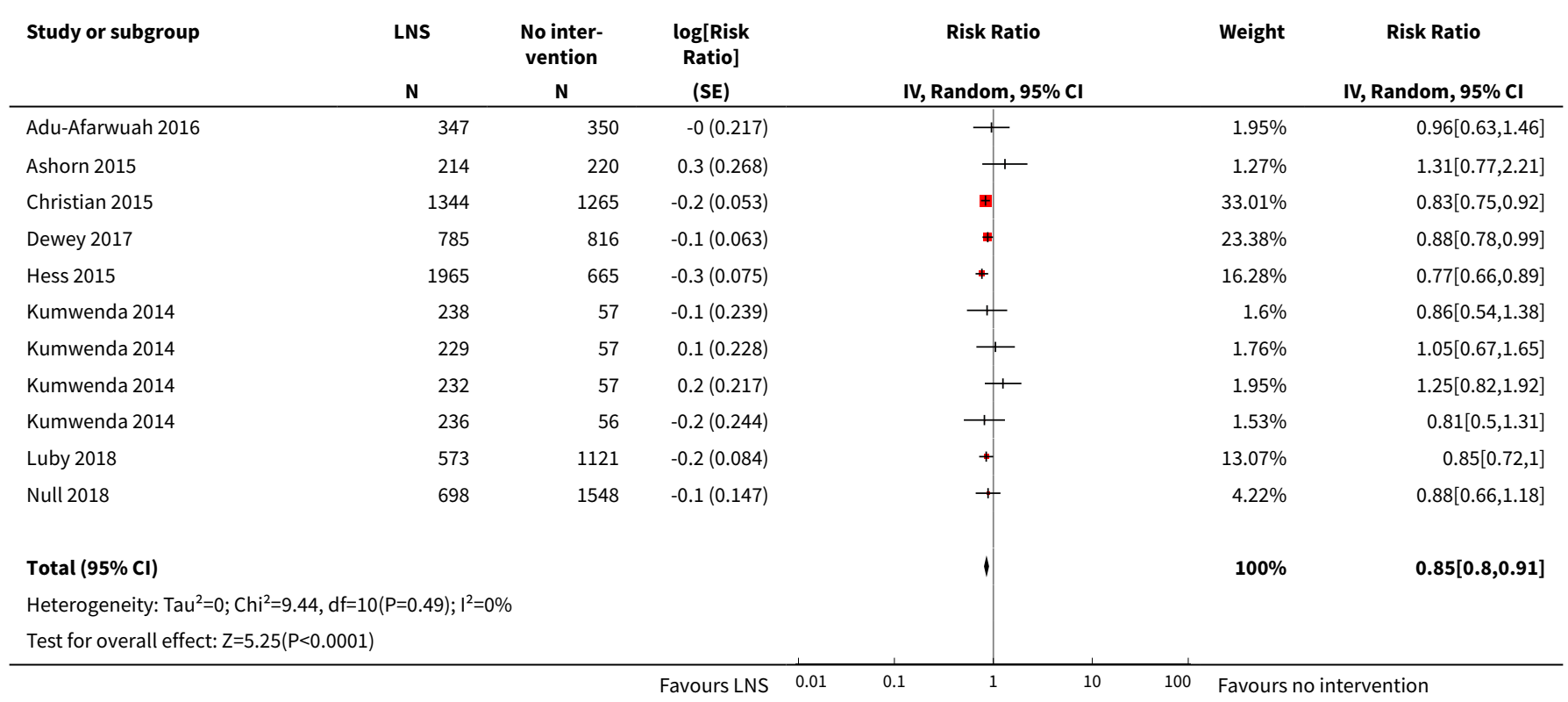


Analysis 1.10. Comparison 1 LNS versus no intervention, Outcome 10 Severe underweight.

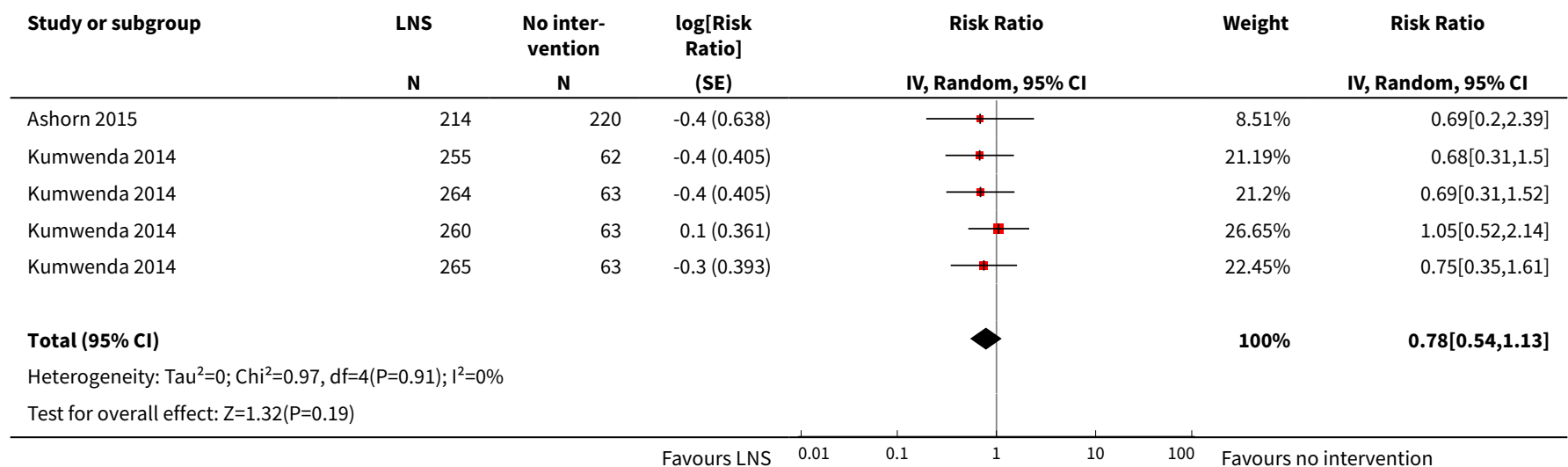

Analysis 1.11. Comparison 1 LNS versus no intervention, Outcome 11 Moderate underweight: Sensitivity analysis.

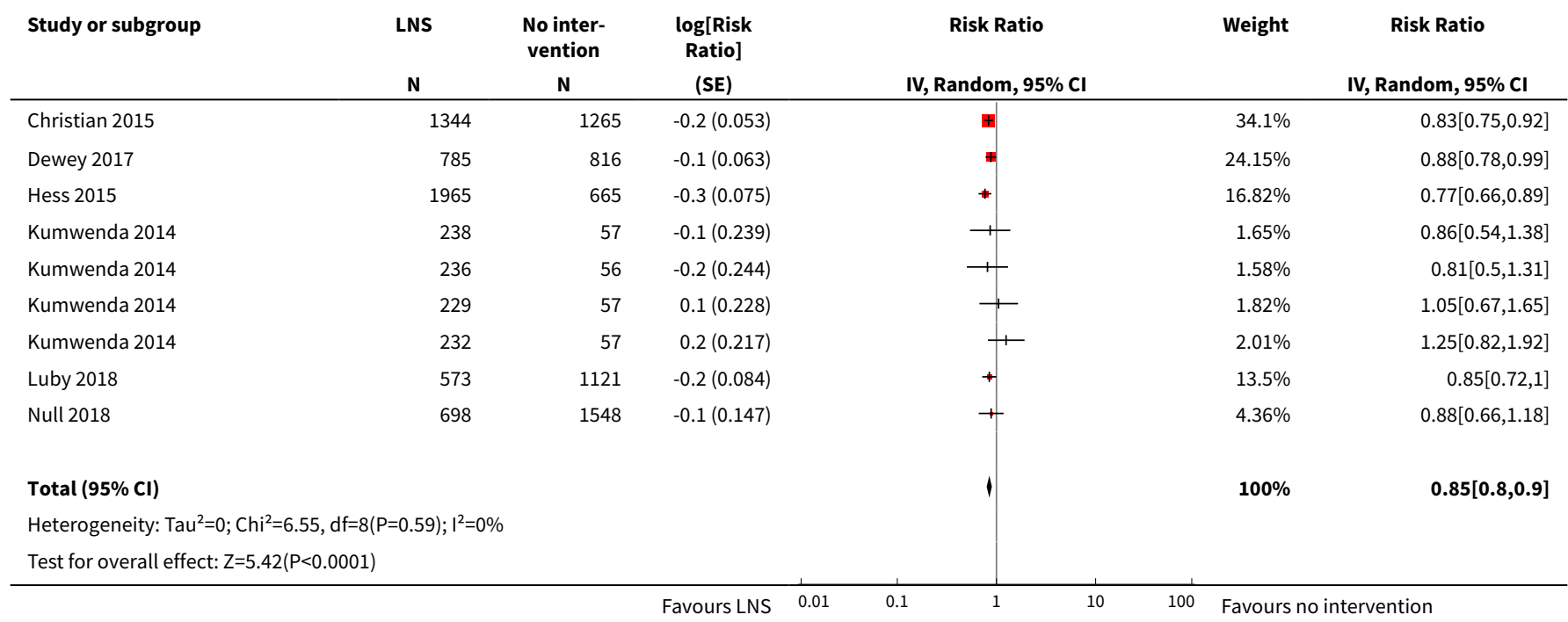

Analysis 1.12. Comparison 1 LNS versus no intervention, Outcome 12 Severe underweight: Sensitivity analysis.

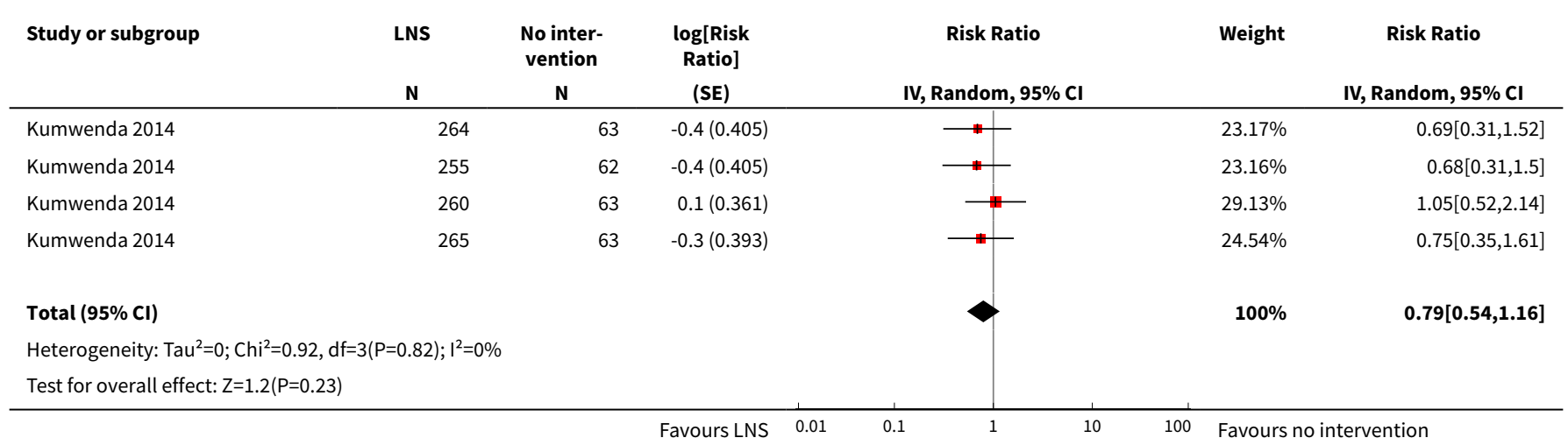

Preventive lipid-based nutrient supplements given with complementary foods to infants and young children 6 to 23 months of age for 
Analysis 1.13. Comparison 1 LNS versus no intervention, Outcome 13 Anaemia.

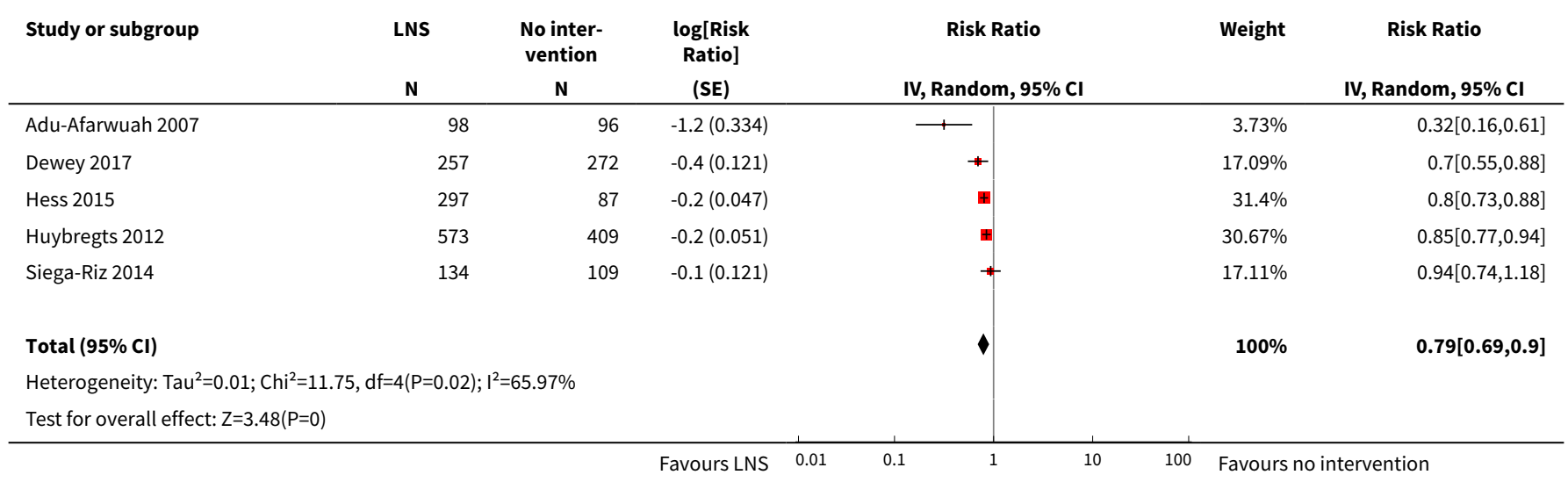

Analysis 1.14. Comparison 1 LNS versus no intervention, Outcome 14 Adverse effects.

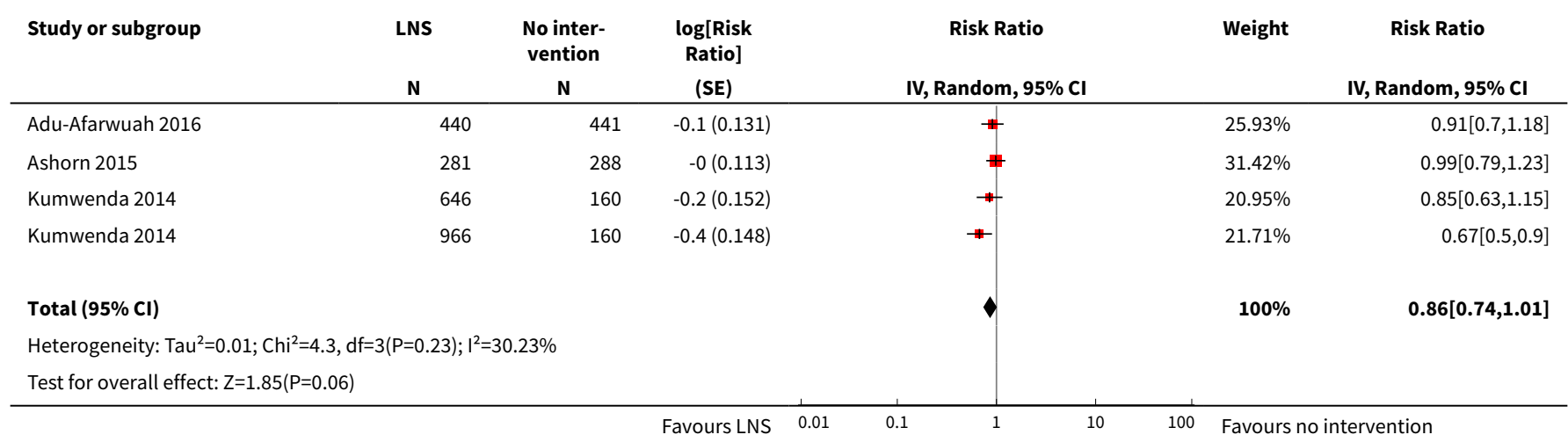

\section{Analysis 1.15. Comparison 1 LNS versus no intervention, Outcome 15 Adverse effects: Sensitivity analysis.}

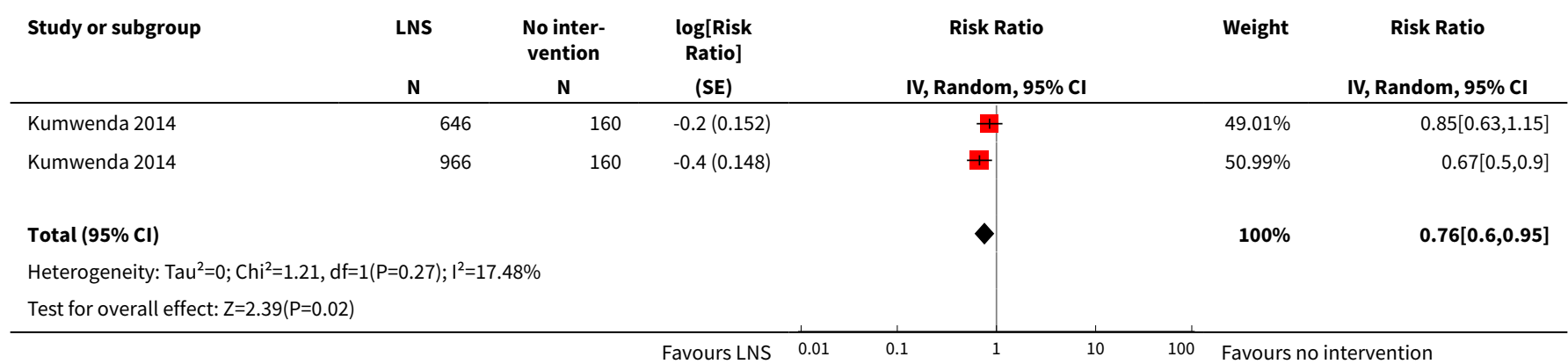

Preventive lipid-based nutrient supplements given with complementary foods to infants and young children 6 to 23 months of age for 
Analysis 1.16. Comparison 1 LNS versus no intervention, Outcome 16 Mid-upper arm circumference (MUAC).

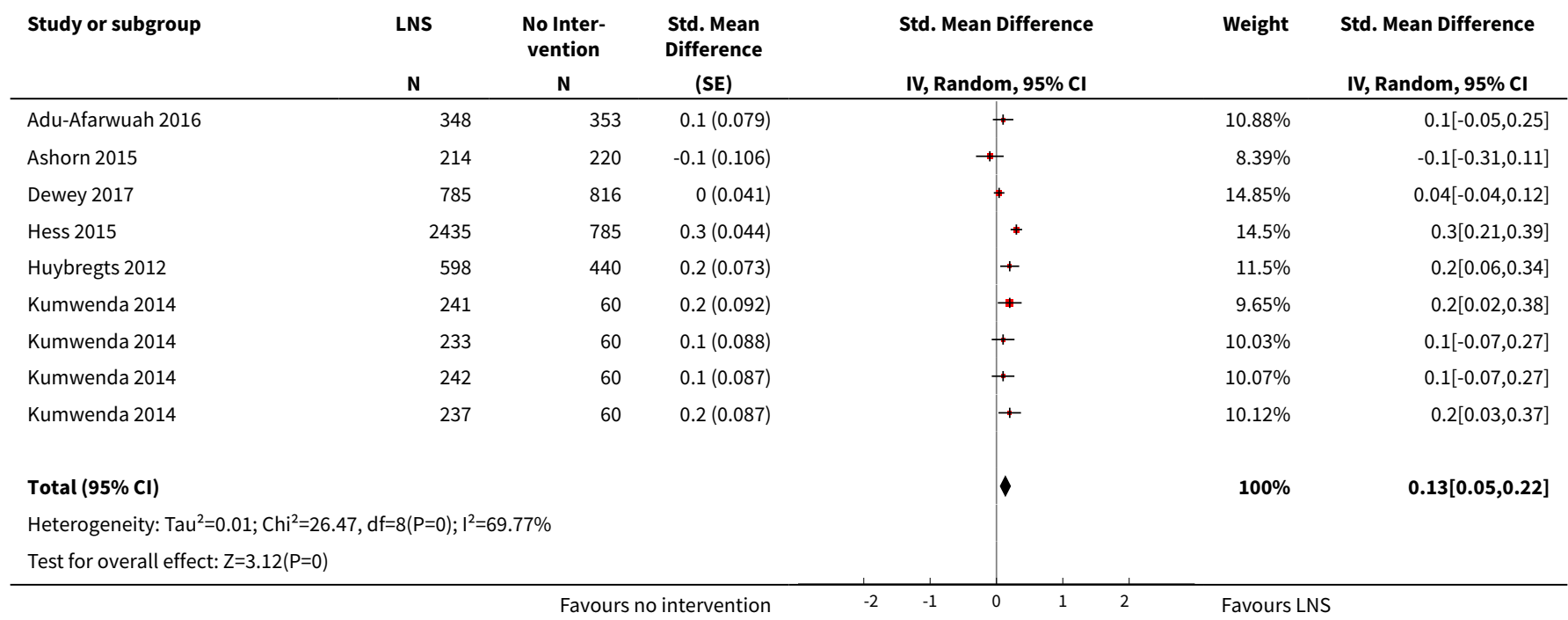

Analysis 1.17. Comparison 1 LNS versus no intervention, Outcome 17 Serum haemoglobin (g/L).

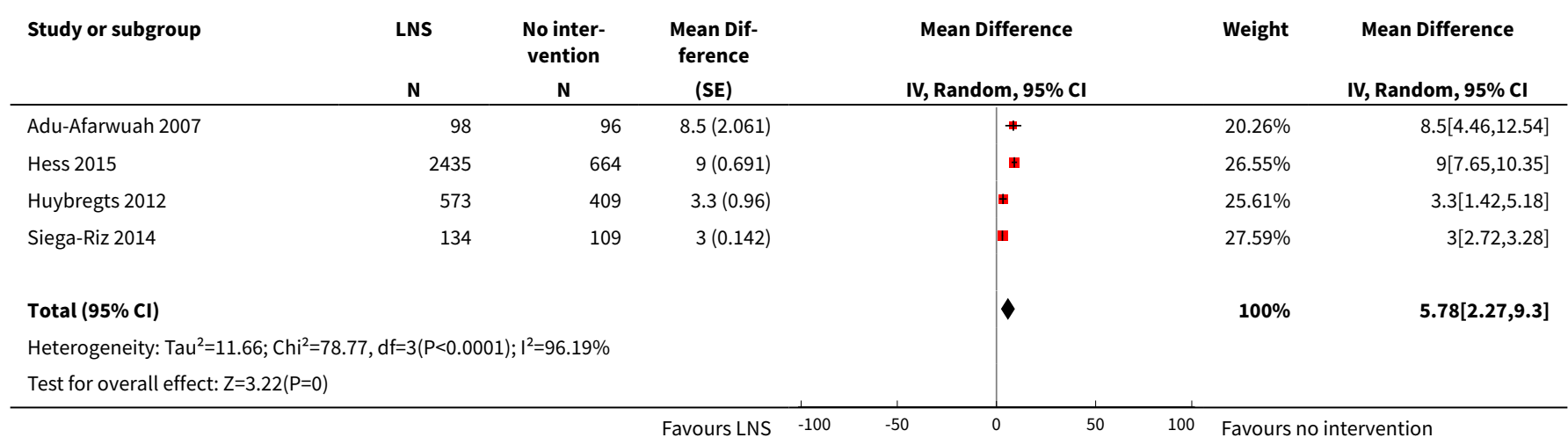

Analysis 1.18. Comparison 1 LNS versus no intervention, Outcome 18 Mortality.

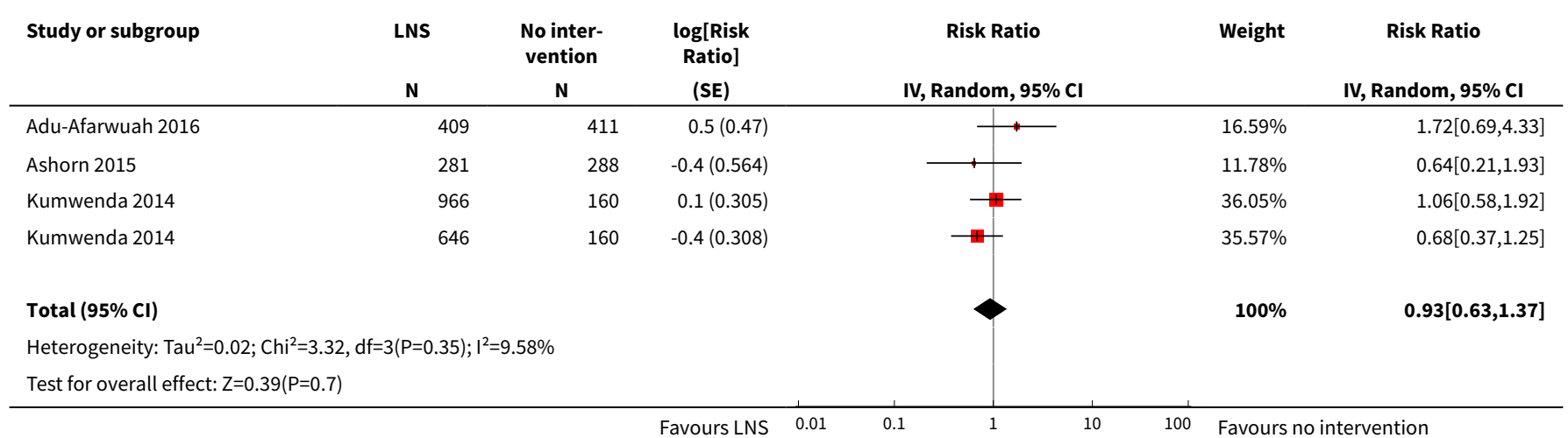

Preventive lipid-based nutrient supplements given with complementary foods to infants and young children 6 to 23 months of age for 
Analysis 1.19. Comparison 1 LNS versus no intervention, Outcome $19 \mathrm{HAZ}$.

\begin{tabular}{|c|c|c|c|c|c|c|}
\hline \multirow{2}{*}{$\begin{array}{l}\text { Study or subgroup } \\
\text { Adu-Afarwuah } 2007\end{array}$} & \multirow{2}{*}{$\begin{array}{l}\text { LNS } \\
\qquad \begin{array}{l}\text { N } \\
\\
97\end{array}\end{array}$} & \multirow{2}{*}{$\begin{array}{l}\begin{array}{l}\text { No inter- } \\
\text { vention } \\
\text { N } \\
\end{array} \quad 81 \\
\end{array}$} & \multirow{2}{*}{$\begin{array}{c}\begin{array}{c}\text { Std. Mean } \\
\text { Difference } \\
\text { (SE) }\end{array} \\
0.3(0.151)\end{array}$} & $\begin{array}{l}\text { Std. Mean Difference } \\
\text { IV, Random, } 95 \% \mathrm{CI}\end{array}$ & \multirow{2}{*}{$\begin{array}{r}\text { Weight } \\
2.67 \%\end{array}$} & \multirow{2}{*}{$\begin{array}{l}\text { Std. Mean Difference } \\
\text { IV, Random, 95\% CI } \\
0.26[-0.03,0.55]\end{array}$} \\
\hline & & & & 1 & & \\
\hline Adu-Afarwuah 2016 & 347 & 350 & $0.2(0.076)$ & $\rightarrow$ & $6 \%$ & $0.18[0.03,0.33]$ \\
\hline Ashorn 2015 & 214 & 220 & $-0.1(0.098)$ & 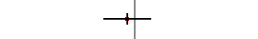 & $4.68 \%$ & $-0.06[-0.25,0.13]$ \\
\hline Christian 2015 & 1344 & 1265 & $0.1(0.039)$ & + & $8.69 \%$ & $0.11[0.03,0.19]$ \\
\hline Hess 2015 & 2435 & 785 & $0.3(0.047)$ & + & $8.12 \%$ & $0.3[0.21,0.39]$ \\
\hline Huybregts 2012 & 598 & 440 & $0.3(0.089)$ & $\rightarrow$ & $5.16 \%$ & $0.27[0.1,0.44]$ \\
\hline Iannotti 2014 & 150 & 144 & $-0(0.14)$ & $\longrightarrow$ & $2.97 \%$ & $-0.02[-0.29,0.25]$ \\
\hline Kumwenda 2014 & 242 & 60 & $0(0.068)$ & + & $6.54 \%$ & $0.01[-0.12,0.14]$ \\
\hline Kumwenda 2014 & 237 & 60 & $0(0.073)$ & + & $6.23 \%$ & $0.03[-0.11,0.17]$ \\
\hline Kumwenda 2014 & 241 & 60 & $-0(0.073)$ & + & $6.2 \%$ & $-0.03[-0.17,0.11]$ \\
\hline Luby 2018 & 567 & 1103 & $0.3(0.054)$ & + & $7.62 \%$ & $0.26[0.15,0.37]$ \\
\hline Mangani 2015 & 212 & 104 & $0(0.072)$ & 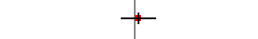 & $6.24 \%$ & $0.03[-0.11,0.17]$ \\
\hline Mangani 2015 & 210 & 105 & $0.1(0.068)$ & + & $6.52 \%$ & $0.08[-0.05,0.21]$ \\
\hline Null 2018 & 695 & 1535 & $0.1(0.051)$ & + & $7.84 \%$ & $0.1[0,0.2]$ \\
\hline Total $(95 \% \mathrm{Cl})$ & & & & 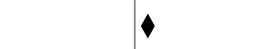 & $100 \%$ & $0.11[0.05,0.16]$ \\
\hline \multicolumn{7}{|c|}{ Heterogeneity: $\mathrm{Tau}^{2}=0.01 ; \mathrm{Chi}^{2}=43.43, \mathrm{df}=15(\mathrm{P}=0) ; \mathrm{I}^{2}=65.46 \%$} \\
\hline \multicolumn{7}{|c|}{ Test for overall effect: $Z=3.69(P=0)$} \\
\hline
\end{tabular}

Analysis 1.20. Comparison 1 LNS versus no intervention, Outcome 20 WAZ.

\begin{tabular}{|c|c|c|c|c|c|c|}
\hline \multirow{2}{*}{$\begin{array}{l}\text { Study or subgroup } \\
\text { Adu-Afarwuah } 2007\end{array}$} & \multirow{2}{*}{$\begin{array}{l}\text { LNS } \\
\begin{array}{l}\text { N } \\
\\
97\end{array}\end{array}$} & \multirow{2}{*}{$\begin{array}{l}\begin{array}{l}\text { No inter- } \\
\text { vention } \\
\mathbf{N}\end{array} \\
81\end{array}$} & \multirow{2}{*}{$\begin{array}{c}\begin{array}{c}\text { Std. Mean } \\
\text { Difference } \\
\text { (SE) }\end{array} \\
0.3(0.166)\end{array}$} & $\begin{array}{l}\text { Std. Mean Difference } \\
\text { IV, Random, } 95 \% \mathrm{CI}\end{array}$ & \multirow{2}{*}{$\begin{array}{r}\text { Weight } \\
3.35 \%\end{array}$} & \multirow{2}{*}{$\begin{array}{l}\text { Std. Mean Difference } \\
\text { IV, Random, 95\% Cl } \\
0.34[0.02,0.66]\end{array}$} \\
\hline & & & & 1 & & \\
\hline Adu-Afarwuah 2016 & 347 & 350 & $0.1(0.078)$ & + & $7.98 \%$ & $0.12[-0.03,0.27]$ \\
\hline Ashorn 2015 & 214 & 220 & $-0.1(0.095)$ & $\rightarrow$ & $6.72 \%$ & $-0.12[-0.31,0.07]$ \\
\hline Dewey 2017 & 785 & 816 & $0.1(0.047)$ & + & $10.66 \%$ & $0.1[0.01,0.19]$ \\
\hline Hess 2015 & 2435 & 785 & $0.3(0.045)$ & + & $10.82 \%$ & $0.28[0.19,0.37]$ \\
\hline lannotti 2014 & 150 & 144 & $0.1(0.131)$ & 1 & $4.63 \%$ & $0.05[-0.21,0.31]$ \\
\hline Kumwenda 2014 & 233 & 60 & $-0(0.133)$ & $\longrightarrow$ & $4.53 \%$ & $-0.04[-0.3,0.22]$ \\
\hline Kumwenda 2014 & 241 & 60 & $-0(0.132)$ & - & $4.61 \%$ & $-0.04[-0.3,0.22]$ \\
\hline Kumwenda 2014 & 242 & 60 & $-0.1(0.133)$ & 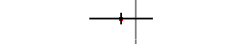 & $4.55 \%$ & $-0.12[-0.38,0.14]$ \\
\hline Kumwenda 2014 & 237 & 60 & $0(0.133)$ & + & $4.55 \%$ & $0[-0.26,0.26]$ \\
\hline Luby 2018 & 573 & 1121 & $0.3(0.054)$ & + & $10.02 \%$ & $0.25[0.14,0.36]$ \\
\hline Mangani 2015 & 212 & 105 & $0.1(0.081)$ & $\rightarrow$ & $7.74 \%$ & $0.05[-0.11,0.21]$ \\
\hline Null 2018 & 698 & 1548 & $0.1(0.037)$ & + & $11.44 \%$ & $0.07[-0,0.14]$ \\
\hline Total $(95 \% \mathrm{Cl})$ & & & & $\downarrow$ & $100 \%$ & $0.09[0.02,0.16]$ \\
\hline \multicolumn{7}{|c|}{ Heterogeneity: $\mathrm{Tau}^{2}=0.01 ; \mathrm{Chi}^{2}=37.11, \mathrm{df}=13(\mathrm{P}=0) ; \mathrm{I}^{2}=64.96 \%$} \\
\hline \multicolumn{4}{|c|}{ Test for overall effect: $Z=2.61(P=0.01)$} & & & \\
\hline
\end{tabular}

Preventive lipid-based nutrient supplements given with complementary foods to infants and young children 6 to 23 months of age for 
Analysis 1.21. Comparison 1 LNS versus no intervention, Outcome $21 \mathrm{WHZ}$.

\begin{tabular}{|c|c|c|c|c|c|c|}
\hline \multirow{3}{*}{$\begin{array}{l}\text { Study or subgroup } \\
\text { Adu-Afarwuah } 2007\end{array}$} & \multirow{3}{*}{$\begin{array}{l}\text { LNS } \\
\begin{array}{l}\text { N } \\
\\
97\end{array}\end{array}$} & \multirow{3}{*}{$\begin{array}{l}\begin{array}{c}\text { No inter- } \\
\text { vention } \\
\text { N }\end{array} \\
\end{array}$} & \multirow{3}{*}{$\begin{array}{c}\begin{array}{c}\text { Std. Mean } \\
\text { Difference } \\
\text { (SE) }\end{array} \\
0.3(0.166)\end{array}$} & \multirow{2}{*}{$\begin{array}{l}\text { Std. Mean Difference } \\
\text { IV, Random, } 95 \% \mathrm{CI}\end{array}$} & \multirow{3}{*}{$\begin{array}{r}\text { Weight } \\
1.88 \%\end{array}$} & \multirow{2}{*}{$\begin{array}{l}\text { Std. Mean Difference } \\
\text { IV, Random, } 95 \% \mathrm{CI}\end{array}$} \\
\hline & & & & & & \\
\hline & & & & 1 & & $0.31[-0.01,0.63]$ \\
\hline Adu-Afarwuah 2016 & 347 & 350 & $0.1(0.077)$ & + & $6.52 \%$ & $0.05[-0.1,0.2]$ \\
\hline Ashorn 2015 & 214 & 220 & $-0.1(0.096)$ & + & $4.75 \%$ & $-0.13[-0.32,0.06]$ \\
\hline Dewey 2017 & 785 & 816 & $0.1(0.044)$ & + & $11.79 \%$ & $0.08[-0.01,0.17]$ \\
\hline Hess 2015 & 2435 & 785 & $0.2(0.041)$ & + & $12.51 \%$ & $0.2[0.12,0.28]$ \\
\hline Huybregts 2012 & 598 & 440 & $0(0.059)$ & * & $8.92 \%$ & $0.04[-0.08,0.16]$ \\
\hline Kumwenda 2014 & 233 & 60 & $0.1(0.086)$ & + & $5.54 \%$ & $0.09[-0.08,0.26]$ \\
\hline Kumwenda 2014 & 241 & 60 & $0.1(0.087)$ & $\leftarrow$ & $5.48 \%$ & $0.14[-0.03,0.31]$ \\
\hline Kumwenda 2014 & 237 & 60 & $0.1(0.086)$ & + & $5.54 \%$ & $0.06[-0.11,0.23]$ \\
\hline Kumwenda 2014 & 242 & 60 & $-0(0.092)$ & $\leftarrow$ & $5.03 \%$ & $-0.01[-0.19,0.17]$ \\
\hline Luby 2018 & 567 & 1104 & $0.2(0.051)$ & + & $10.46 \%$ & $0.17[0.07,0.27]$ \\
\hline Mangani 2015 & 212 & 104 & $0(0.097)$ & + & $4.65 \%$ & $0.04[-0.15,0.23]$ \\
\hline Mangani 2015 & 210 & 105 & $0.1(0.095)$ & + & $4.78 \%$ & $0.09[-0.1,0.28]$ \\
\hline Null 2018 & 695 & 1536 & $0(0.042)$ & + & $12.15 \%$ & $0.03[-0.05,0.11]$ \\
\hline Total $(95 \% \mathrm{Cl})$ & & & & $\downarrow$ & $100 \%$ & $0.08[0.04,0.13]$ \\
\hline \multicolumn{7}{|c|}{ Heterogeneity: $\operatorname{Tau}^{2}=0 ; \mathrm{Chi}^{2}=21.83, \mathrm{df}=13(\mathrm{P}=0.06) ; \mathrm{I}^{2}=40.44 \%$} \\
\hline \multicolumn{7}{|c|}{ Test for overall effect: $\mathrm{Z}=3.51(\mathrm{P}=0)$} \\
\hline
\end{tabular}

Comparison 2. LNS versus no intervention: Subgroup analysis by energy content/formulation of product provided

\begin{tabular}{|c|c|c|c|c|}
\hline $\begin{array}{l}\text { Outcome or sub- } \\
\text { group title }\end{array}$ & No. of studies & $\begin{array}{l}\text { No. of partici- } \\
\text { pants }\end{array}$ & Statistical method & Effect size \\
\hline 1 Severe stunting & 5 & 6151 & Risk Ratio (Random, 95\% Cl) & $0.85[0.74,0.98]$ \\
\hline 1.1 SQ LNS & 4 & 4956 & Risk Ratio (Random, 95\% Cl) & $0.83[0.70,0.99]$ \\
\hline $1.2 \mathrm{MQ}$ LNS & 2 & 1195 & Risk Ratio (Random, 95\% Cl) & $0.88[0.69,1.12]$ \\
\hline $\begin{array}{l}2 \text { Moderate stunt- } \\
\text { ing }\end{array}$ & 9 & 13372 & Risk Ratio (Random, 95\% Cl) & $0.93[0.88,0.98]$ \\
\hline 2.1 SQ LNS & 7 & 9710 & Risk Ratio (Random, 95\% Cl) & $0.92[0.84,1.00]$ \\
\hline 2.2 MQ LNS & 3 & 3662 & Risk Ratio (Random, 95\% Cl) & $0.94[0.88,1.02]$ \\
\hline 3 Moderate wasting & 8 & 13172 & Risk Ratio (Random, 95\% Cl) & $0.82[0.74,0.91]$ \\
\hline 3.1 SQ LNS & 7 & 9903 & Risk Ratio (Random, 95\% Cl) & $0.83[0.73,0.95]$ \\
\hline 3.2 MQ LNS & 2 & 3269 & Risk Ratio (Random, 95\% Cl) & $0.78[0.63,0.96]$ \\
\hline 4 Severe wasting & 3 & 2329 & Risk Ratio (Random, 95\% Cl) & $1.27[0.66,2.46]$ \\
\hline 4.1 SQ LNS & 2 & 1106 & Risk Ratio (Random, 95\% Cl) & $1.74[0.73,4.15]$ \\
\hline
\end{tabular}

Preventive lipid-based nutrient supplements given with complementary foods to infants and young children 6 to 23 months of age for 


\begin{tabular}{|c|c|c|c|c|}
\hline $\begin{array}{l}\text { Outcome or sub- } \\
\text { group title }\end{array}$ & No. of studies & $\begin{array}{l}\text { No. of partici- } \\
\text { pants }\end{array}$ & Statistical method & Effect size \\
\hline 4.2 MQ LNS & 2 & 1223 & Risk Ratio (Random, 95\% Cl) & $0.84[0.31,2.30]$ \\
\hline $\begin{array}{l}5 \text { Moderate under- } \\
\text { weight }\end{array}$ & 8 & 13073 & Risk Ratio (Random, 95\% Cl) & $0.85[0.80,0.91]$ \\
\hline 5.1 SQ LNS & 7 & 9880 & Risk Ratio (Random, 95\% Cl) & $0.88[0.80,0.96]$ \\
\hline $5.2 \mathrm{MQ}$ LNS & 2 & 3193 & Risk Ratio (Random, 95\% Cl) & $0.83[0.76,0.92]$ \\
\hline $\begin{array}{l}6 \text { Severe under- } \\
\text { weight }\end{array}$ & 2 & 1729 & Risk Ratio (Random, 95\% Cl) & $0.78[0.54,1.13]$ \\
\hline 6.1 SQ LNS & 2 & 1083 & Risk Ratio (Random, 95\% Cl) & $0.84[0.52,1.37]$ \\
\hline $6.2 \mathrm{MQ}$ LNS & 1 & 646 & Risk Ratio (Random, 95\% Cl) & $0.71[0.41,1.24]$ \\
\hline 7 Anaemia & 5 & 2332 & Risk Ratio (M-H, Random, 95\% Cl) & $0.79[0.69,0.90]$ \\
\hline 7.1 SQ LNS & 3 & 1107 & Risk Ratio $(\mathrm{M}-\mathrm{H}$, Random, 95\% Cl) & $0.63[0.43,0.93]$ \\
\hline $7.2 \mathrm{MQ}$ LNS & 2 & 1225 & Risk Ratio (M-H, Random, 95\% Cl) & $0.86[0.78,0.94]$ \\
\hline 8 Adverse effects & 3 & 3382 & Risk Ratio (Random, 95\% Cl) & $0.86[0.74,1.01]$ \\
\hline 8.1 SQ LNS & 3 & 2576 & Risk Ratio (Random, 95\% Cl) & $0.86[0.69,1.06]$ \\
\hline 8.2 MQ LNS & 1 & 806 & Risk Ratio (Random, 95\% Cl) & $0.85[0.63,1.15]$ \\
\hline $\begin{array}{l}9 \text { Mid-upper arm } \\
\text { circumference } \\
\text { (MUAC) }\end{array}$ & 6 & 8187 & Std. Mean Difference (Random, 95\% Cl) & $0.13[0.05,0.22]$ \\
\hline 9.1 SQ LNS & 5 & 6546 & Std. Mean Difference (Random, 95\% Cl) & $0.12[-0.00,0.24]$ \\
\hline 9.2 MQ LNS & 2 & 1641 & Std. Mean Difference (Random, 95\% Cl) & $0.17[0.08,0.26]$ \\
\hline $\begin{array}{l}10 \text { Serum haemo- } \\
\text { globin }(\mathrm{g} / \mathrm{L})\end{array}$ & 4 & 4518 & Mean Difference (IV, Random, 95\% CI) & $5.78[2.27,9.30]$ \\
\hline 10.1 SQ LNS & 2 & 3293 & Mean Difference (IV, Random, 95\% CI) & $8.95[7.66,10.23]$ \\
\hline $10.2 \mathrm{MQ}$ LNS & 2 & 1225 & Mean Difference (IV, Random, 95\% CI) & $3.01[2.73,3.28]$ \\
\hline 11 Mortality & 3 & 3321 & Risk Ratio (Random, 95\% Cl) & $0.93[0.63,1.37]$ \\
\hline 11.1 SQ LNS & 3 & 2195 & Risk Ratio (Random, 95\% Cl) & $0.88[0.49,1.60]$ \\
\hline $11.2 \mathrm{MQ}$ LNS & 1 & 1126 & Risk Ratio (Random, 95\% Cl) & $1.06[0.58,1.92]$ \\
\hline $12 \mathrm{HAZ}$ & 12 & 15795 & Std. Mean Difference (Random, 95\% Cl) & $0.11[0.05,0.16]$ \\
\hline 12.1 SQ LNS & 9 & 10919 & Std. Mean Difference (Random, 95\% Cl) & $0.12[0.04,0.20]$ \\
\hline 12.2 MQ LNS & 4 & 4876 & Std. Mean Difference (Random, 95\% Cl) & $0.08[0.01,0.15]$ \\
\hline
\end{tabular}

Preventive lipid-based nutrient supplements given with complementary foods to infants and young children 6 to 23 months of age for 


\begin{tabular}{lllll}
\hline $\begin{array}{l}\text { Outcome or sub- } \\
\text { group title }\end{array}$ & No. of studies & $\begin{array}{l}\text { No. of partici- } \\
\text { pants }\end{array}$ & Statistical method & Effect size \\
\hline $13 \mathrm{WHZ}$ & 10 & 12894 & Std. Mean Difference (Random, 95\% Cl) & $0.08[0.04,0.13]$ \\
\hline 13.1 SQ LNS & 8 & 10631 & Std. Mean Difference (Random, 95\% Cl) & $0.08[0.02,0.15]$ \\
\hline 13.2 MQ LNS & 3 & 2263 & Std. Mean Difference (Random, 95\% Cl) & $0.07[0.00,0.14]$ \\
\hline 14 WAZ & 10 & 12188 & Std. Mean Difference (Random, 95\% Cl) & $0.09[0.02,0.16]$ \\
\hline 14.1 SQ LNS & 9 & 10959 & Std. Mean Difference (Random, 95\% Cl) & $0.11[0.02,0.19]$ \\
\hline 14.2 MQ LNS & 2 & 1229 & Std. Mean Difference (Random, 95\% Cl) & $0.05[-0.04,0.14]$ \\
\hline
\end{tabular}

Analysis 2.1. Comparison 2 LNS versus no intervention: Subgroup analysis by energy content/formulation of product provided, Outcome 1 Severe stunting.

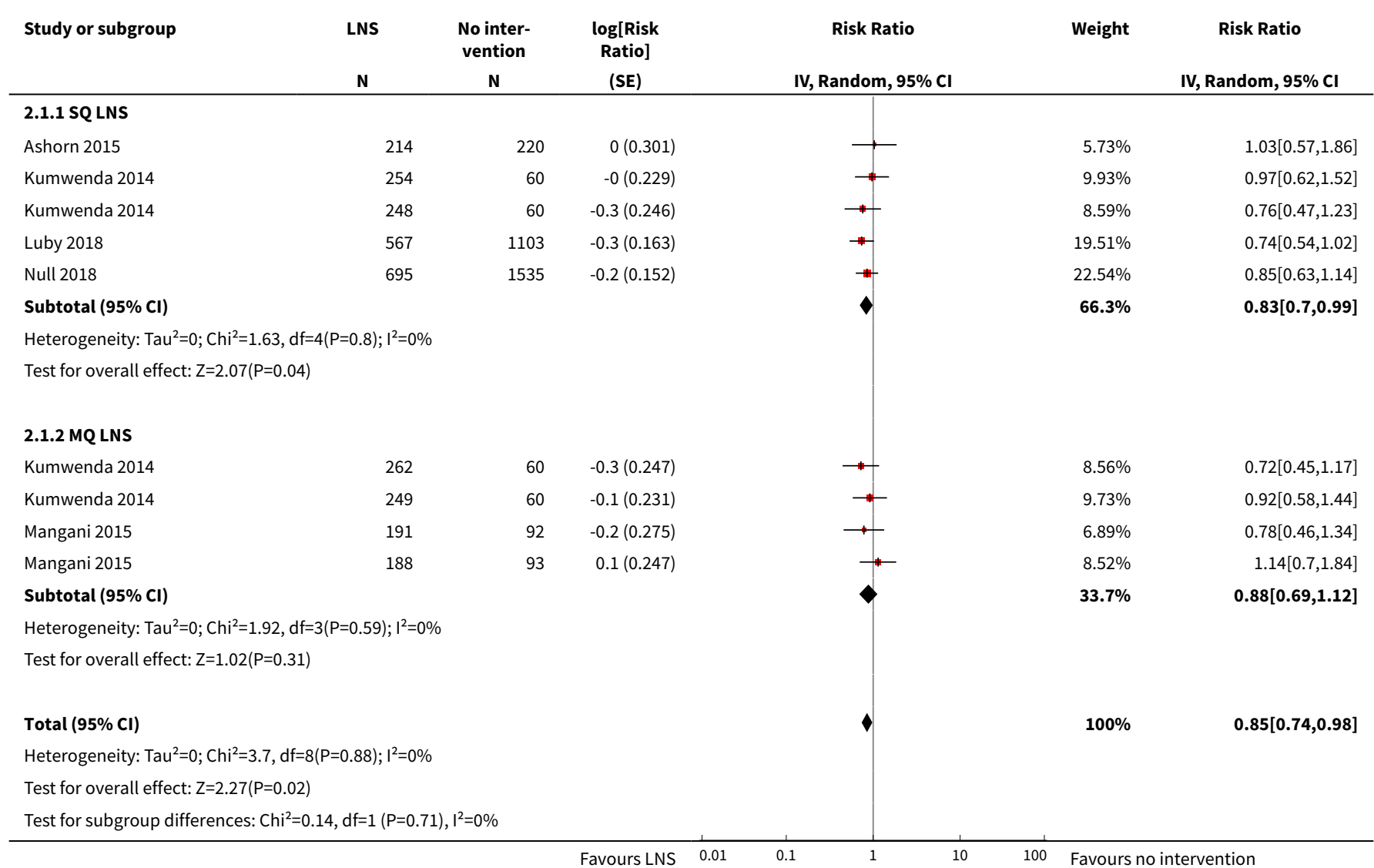


Analysis 2.2. Comparison 2 LNS versus no intervention: Subgroup analysis by energy content/formulation of product provided, Outcome 2 Moderate stunting.

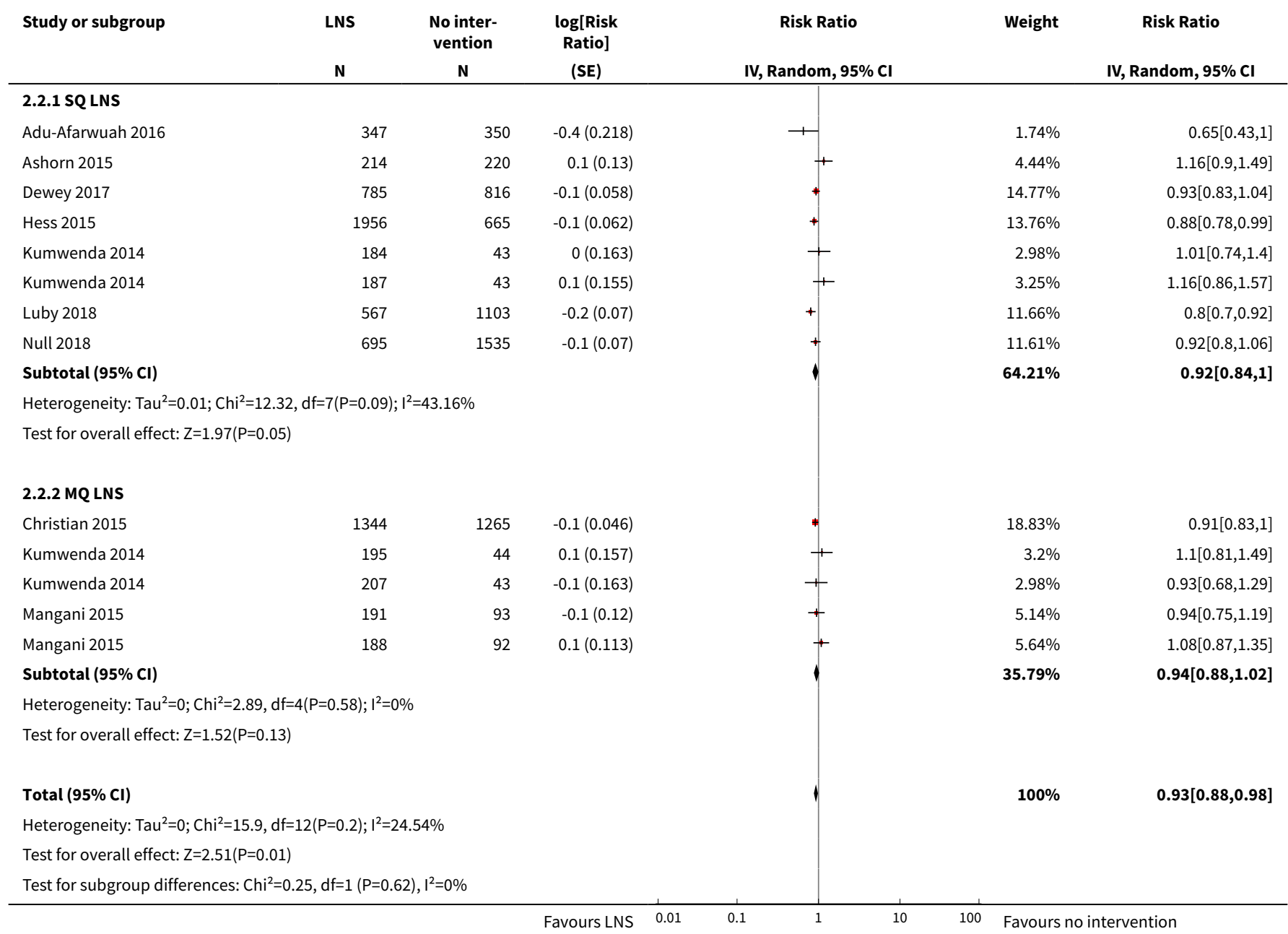

Analysis 2.3. Comparison 2 LNS versus no intervention: Subgroup analysis by energy content/formulation of product provided, Outcome 3 Moderate wasting.

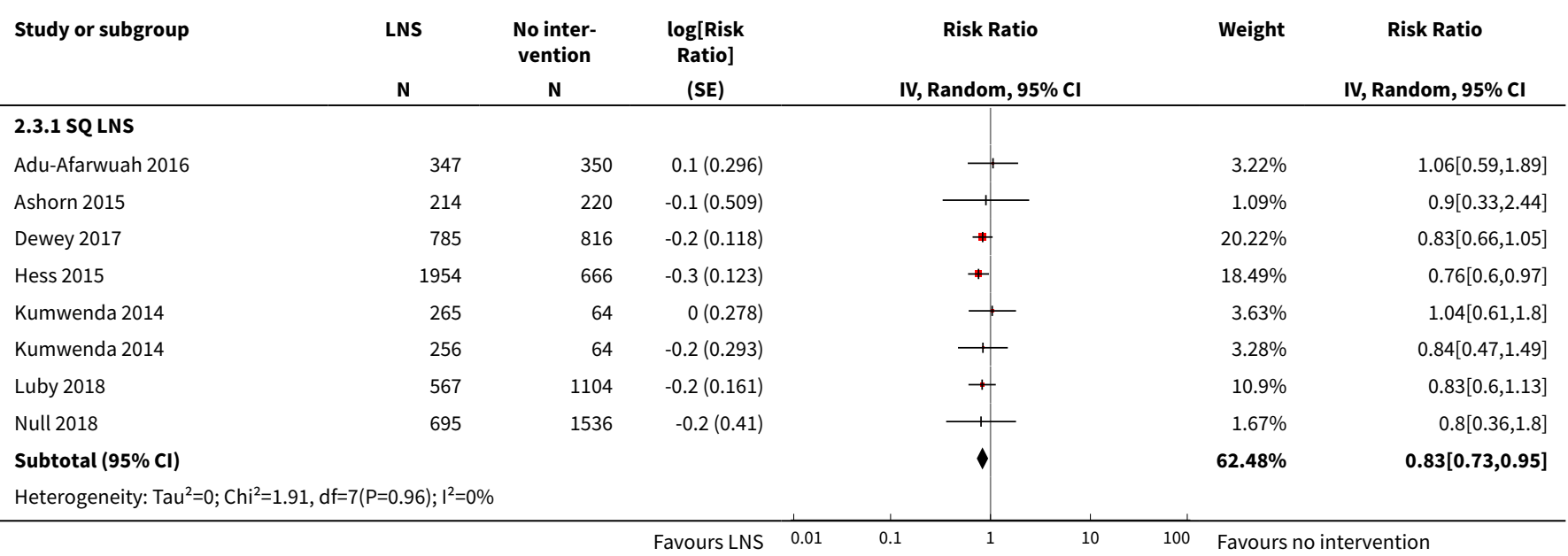

Preventive lipid-based nutrient supplements given with complementary foods to infants and young children 6 to 23 months of age for 89 health, nutrition, and developmental outcomes (Review)

Copyright (c) 2019 The Cochrane Collaboration. Published by John Wiley \& Sons, Ltd. 


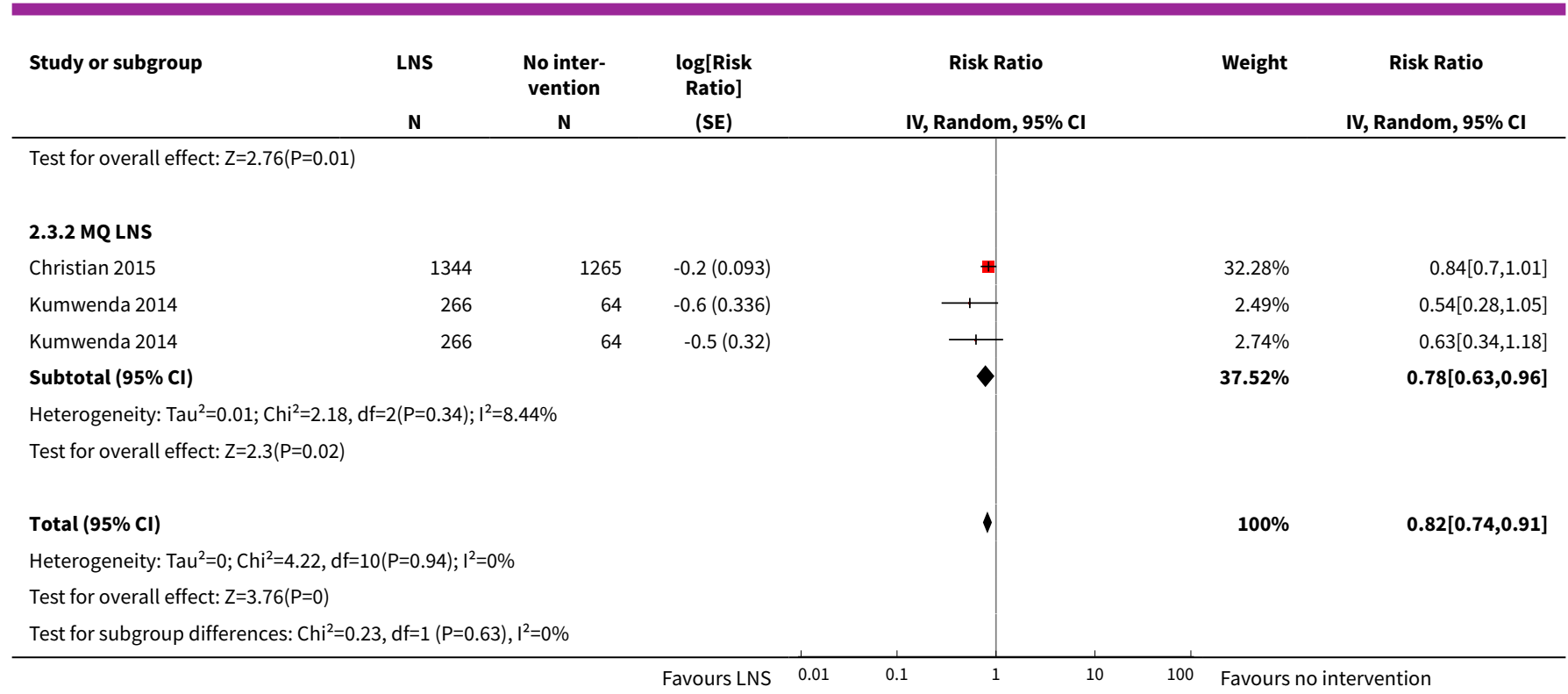

\section{Analysis 2.4. Comparison 2 LNS versus no intervention: Subgroup analysis by energy content/formulation of product provided, Outcome 4 Severe wasting.}

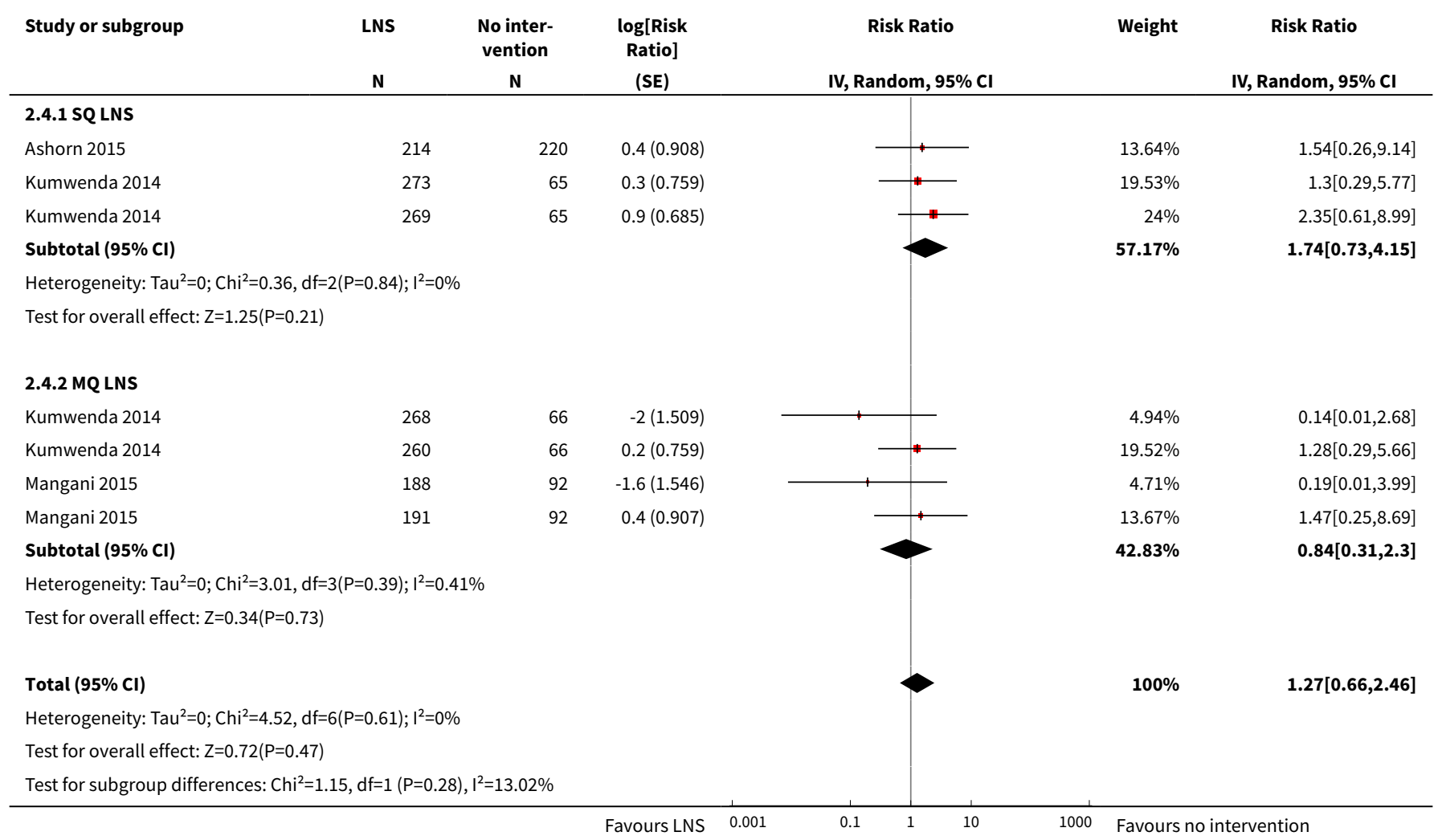

Preventive lipid-based nutrient supplements given with complementary foods to infants and young children 6 to 23 months of age for 
Analysis 2.5. Comparison 2 LNS versus no intervention: Subgroup analysis by energy content/formulation of product provided, Outcome 5 Moderate underweight.

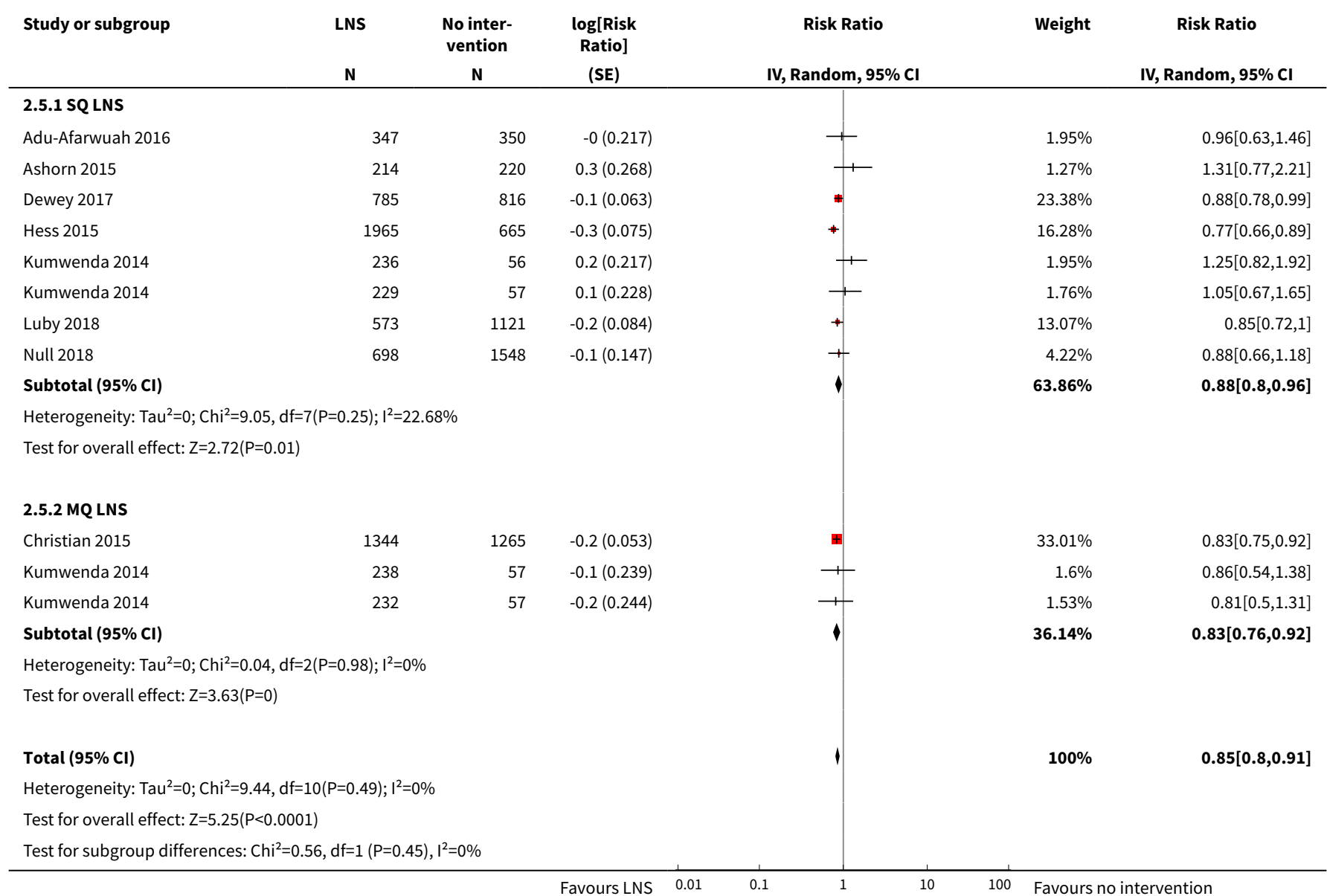

Analysis 2.6. Comparison 2 LNS versus no intervention: Subgroup analysis by energy content/formulation of product provided, Outcome 6 Severe underweight.

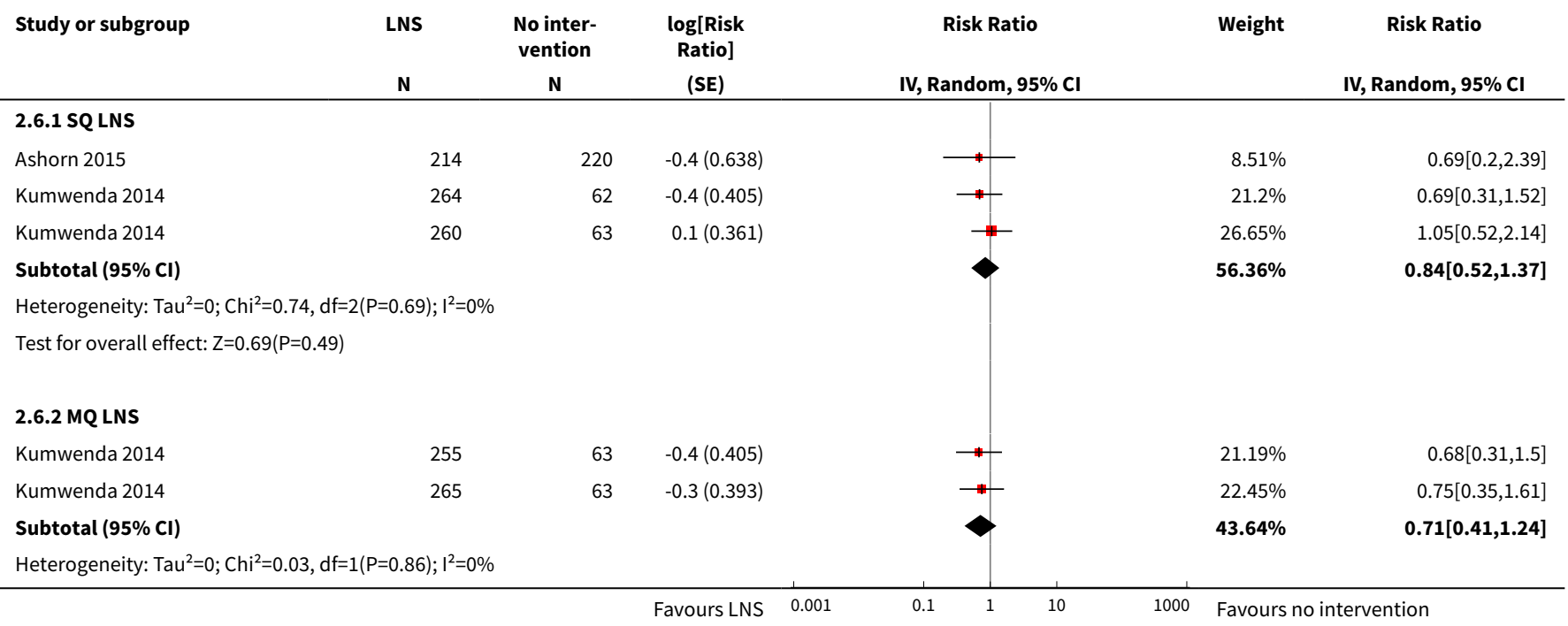

Preventive lipid-based nutrient supplements given with complementary foods to infants and young children 6 to 23 months of age for 


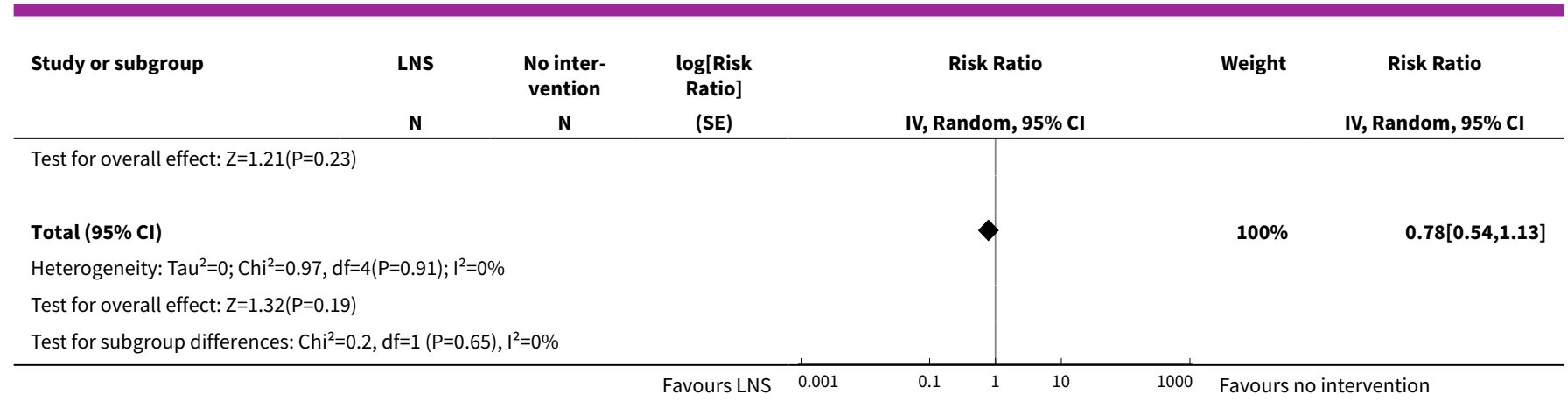

Analysis 2.7. Comparison 2 LNS versus no intervention: Subgroup analysis by energy content/formulation of product provided, Outcome 7 Anaemia.

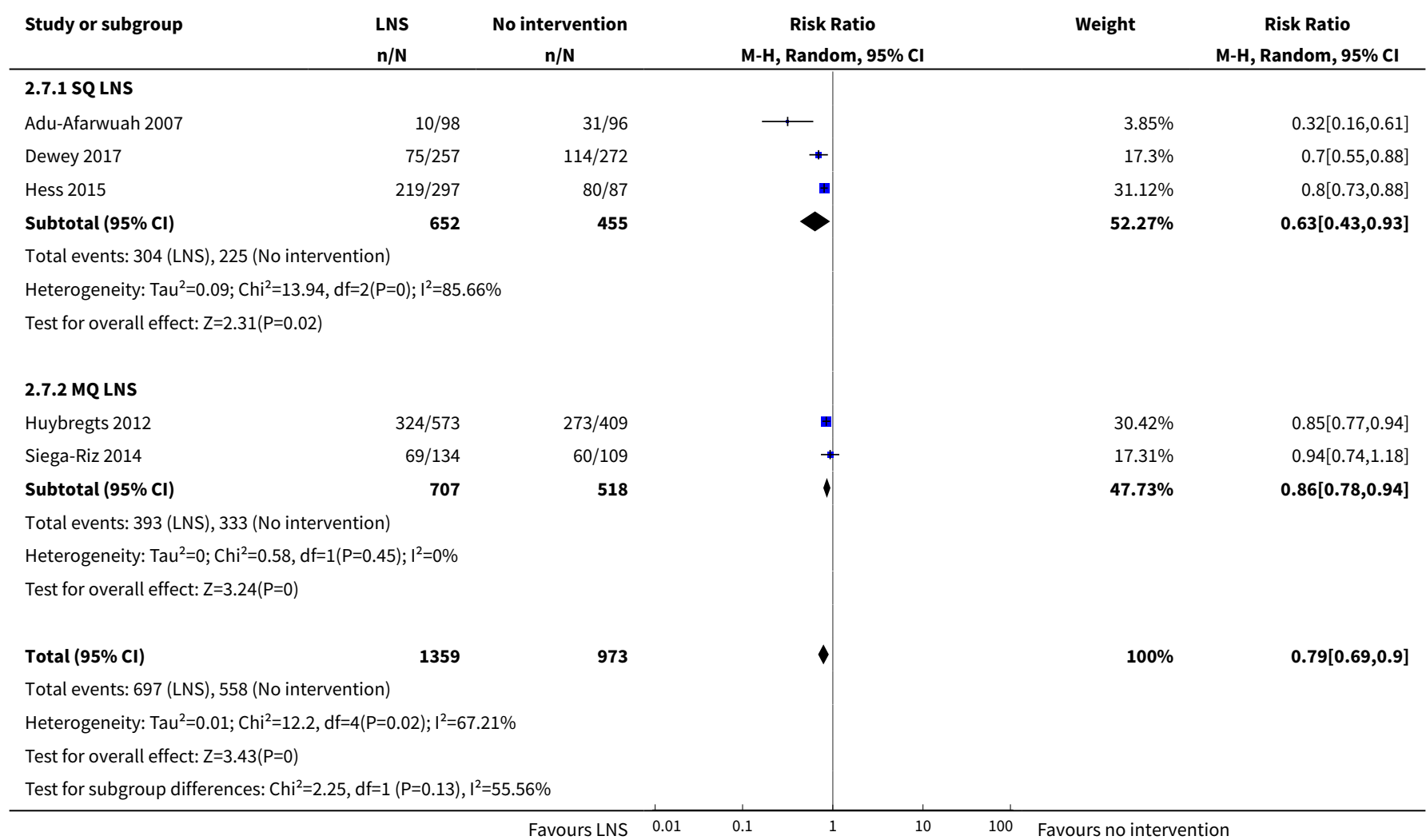

Analysis 2.8. Comparison 2 LNS versus no intervention: Subgroup analysis by energy content/formulation of product provided, Outcome 8 Adverse effects.

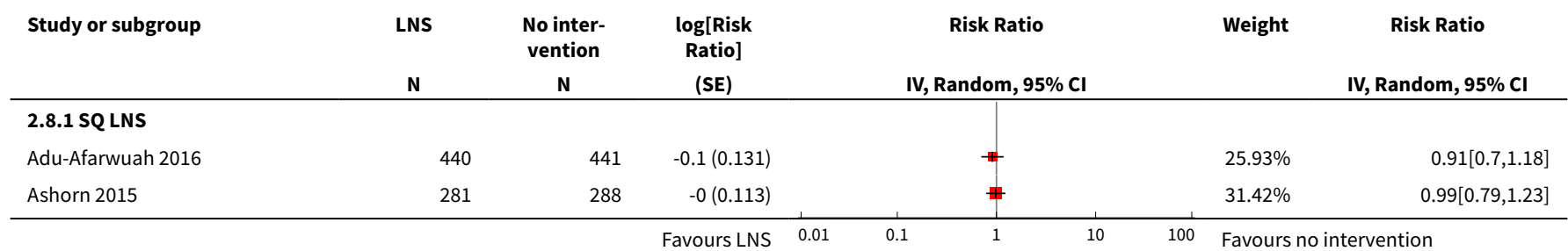




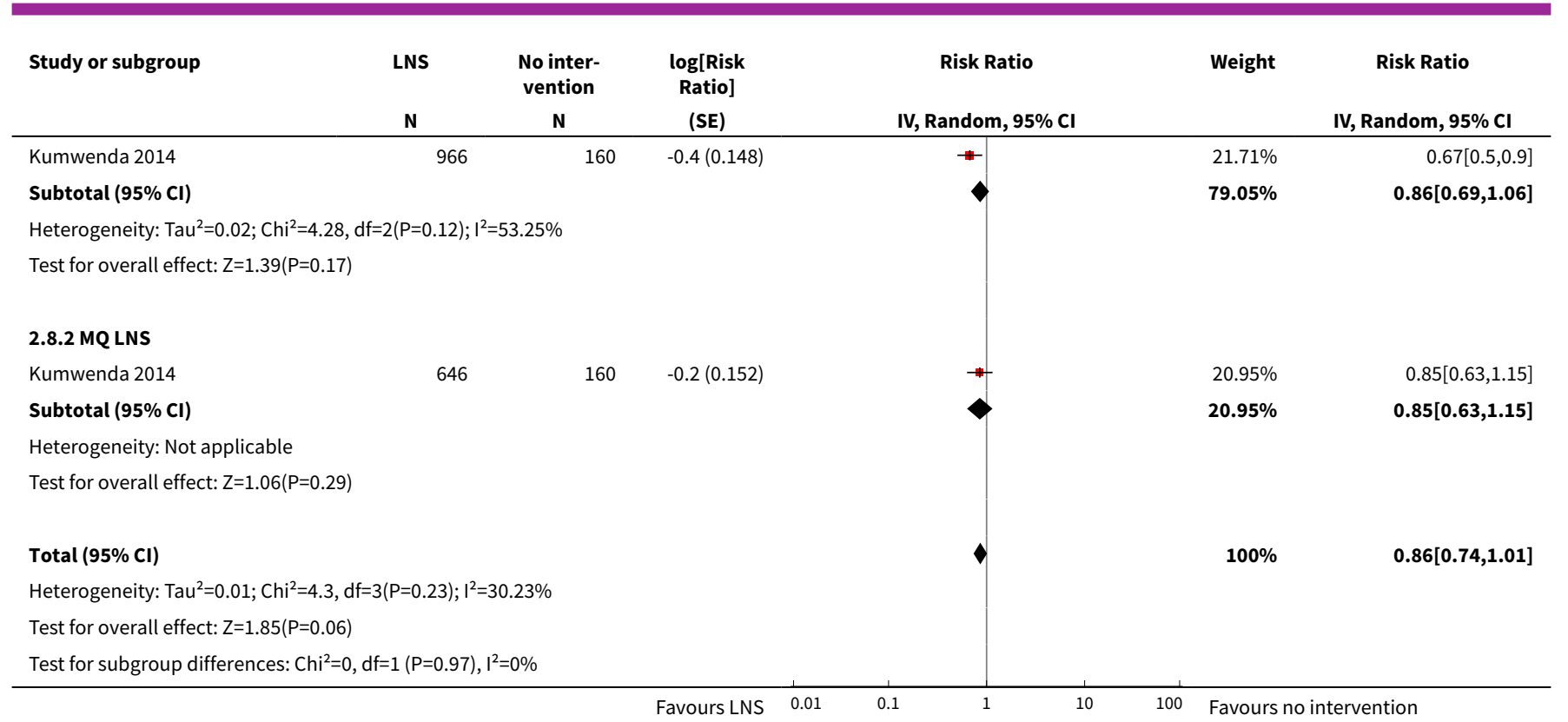

\section{Analysis 2.9. Comparison 2 LNS versus no intervention: Subgroup analysis by energy content/formulation of product provided, Outcome 9 Mid-upper arm circumference (MUAC).}

\begin{tabular}{|c|c|c|c|c|c|c|}
\hline Study or subgroup & $\begin{array}{l}\text { LNS } \\
\mathrm{N} \\
\end{array}$ & $\begin{array}{c}\text { No inter- } \\
\text { vention } \\
\mathbf{N} \\
\end{array}$ & $\begin{array}{l}\text { Std. Mean } \\
\text { Difference } \\
\text { (SE) } \\
\end{array}$ & $\begin{array}{l}\text { Std. Mean Difference } \\
\text { IV, Random, } 95 \% \mathrm{CI}\end{array}$ & Weight & $\begin{array}{l}\text { Std. Mean Difference } \\
\text { IV, Random, } 95 \% \mathrm{CI}\end{array}$ \\
\hline \multicolumn{7}{|l|}{ 2.9.1 SQ LNS } \\
\hline Adu-Afarwuah 2016 & 348 & 353 & $0.1(0.079)$ & + & $10.88 \%$ & $0.1[-0.05,0.25]$ \\
\hline Ashorn 2015 & 214 & 220 & $-0.1(0.106)$ & + & $8.39 \%$ & $-0.1[-0.31,0.11]$ \\
\hline Dewey 2017 & 785 & 816 & $0(0.041)$ & + & $14.85 \%$ & $0.04[-0.04,0.12]$ \\
\hline Kumwenda 2014 & 233 & 60 & $0.1(0.088)$ & + & $10.03 \%$ & $0.1[-0.07,0.27]$ \\
\hline Kumwenda 2014 & 237 & 60 & $0.2(0.087)$ & + & $10.12 \%$ & $0.2[0.03,0.37]$ \\
\hline Subtotal $(95 \% \mathrm{Cl})$ & & & & $\checkmark$ & $68.77 \%$ & $0.12[-0,0.24]$ \\
\hline \multicolumn{7}{|c|}{ Heterogeneity: $\operatorname{Tau}^{2}=0.02 ; \mathrm{Chi}^{2}=25.19, \mathrm{df}=5(\mathrm{P}=0) ; \mathrm{I}^{2}=80.15 \%$} \\
\hline \multicolumn{7}{|c|}{ Test for overall effect: $Z=1.91(P=0.06)$} \\
\hline Huybregts 2012 & 598 & 440 & $0.2(0.073)$ & + & $11.5 \%$ & $0.2[0.06,0.34]$ \\
\hline Kumwenda 2014 & 242 & 60 & $0.1(0.087)$ & + & $10.07 \%$ & $0.1[-0.07,0.27]$ \\
\hline Kumwenda 2014 & 241 & 60 & $0.2(0.092)$ & * & $9.65 \%$ & $0.2[0.02,0.38]$ \\
\hline Subtotal $(95 \% \mathrm{Cl})$ & & & & $\downarrow$ & $31.23 \%$ & $0.17[0.08,0.26]$ \\
\hline \multicolumn{7}{|c|}{ Heterogeneity: $\operatorname{Tau}^{2}=0 ; \mathrm{Chi}^{2}=0.92, \mathrm{df}=2(\mathrm{P}=0.63) ; \mathrm{I}^{2}=0 \%$} \\
\hline \multicolumn{7}{|c|}{ Test for overall effect: $Z=3.57(P=0)$} \\
\hline Total $(95 \% \mathrm{Cl})$ & & & & 1 & $100 \%$ & $0.13[0.05,0.22]$ \\
\hline \multicolumn{7}{|c|}{ Heterogeneity: $\operatorname{Tau}^{2}=0.01 ; \mathrm{Chi}^{2}=26.47, \mathrm{df}=8(\mathrm{P}=0) ; \mathrm{I}^{2}=69.77 \%$} \\
\hline \multicolumn{7}{|c|}{ Test for overall effect: $Z=3.12(P=0)$} \\
\hline Test for subgroup diff & $d f=1(P=0$. & ${ }^{2}=0 \%$ & & & & \\
\hline
\end{tabular}


Analysis 2.10. Comparison 2 LNS versus no intervention: Subgroup analysis by energy content/formulation of product provided, Outcome 10 Serum haemoglobin $(\mathrm{g} / \mathrm{L})$.

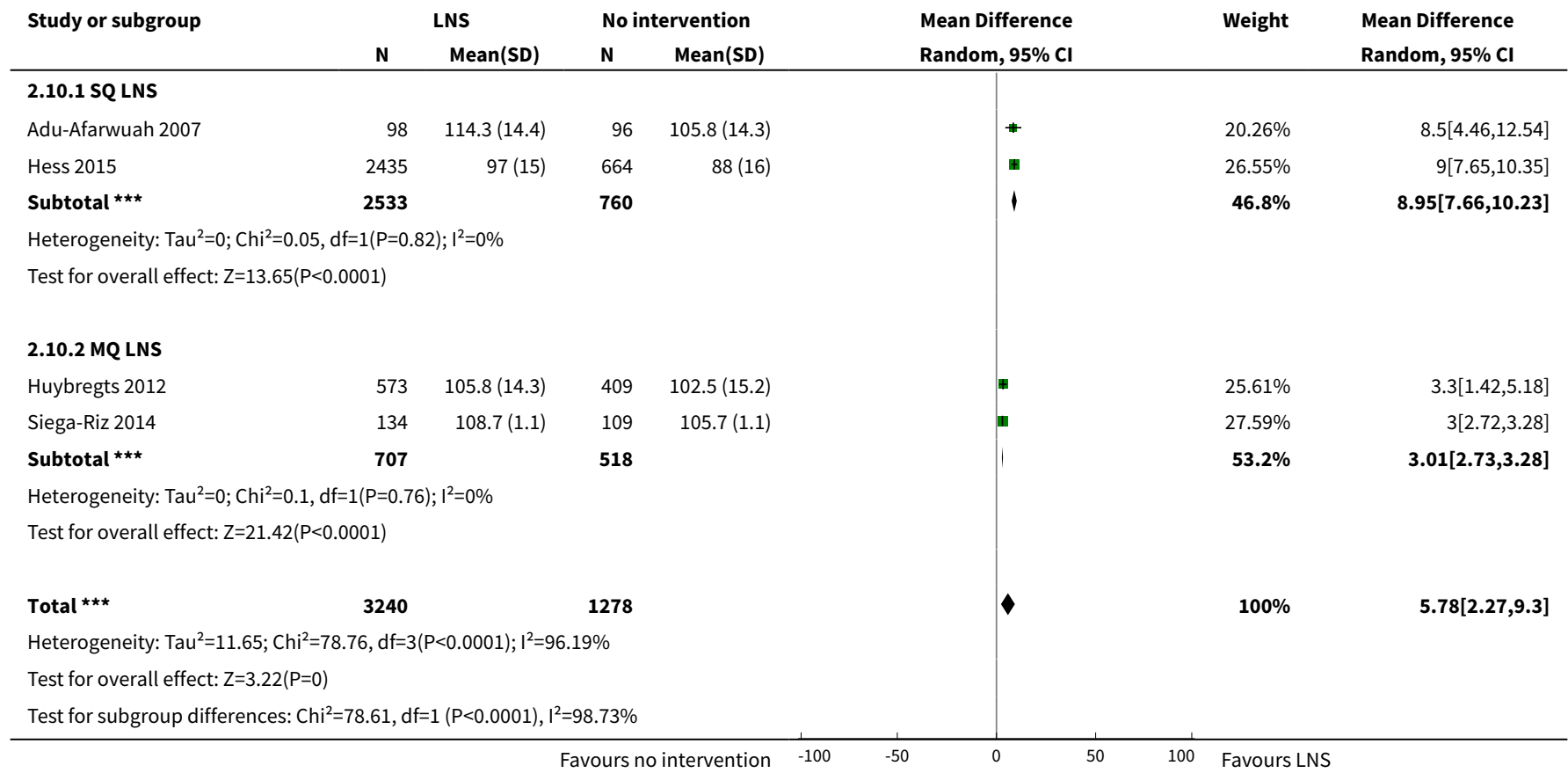

Analysis 2.11. Comparison 2 LNS versus no intervention: Subgroup analysis by energy content/formulation of product provided, Outcome 11 Mortality.

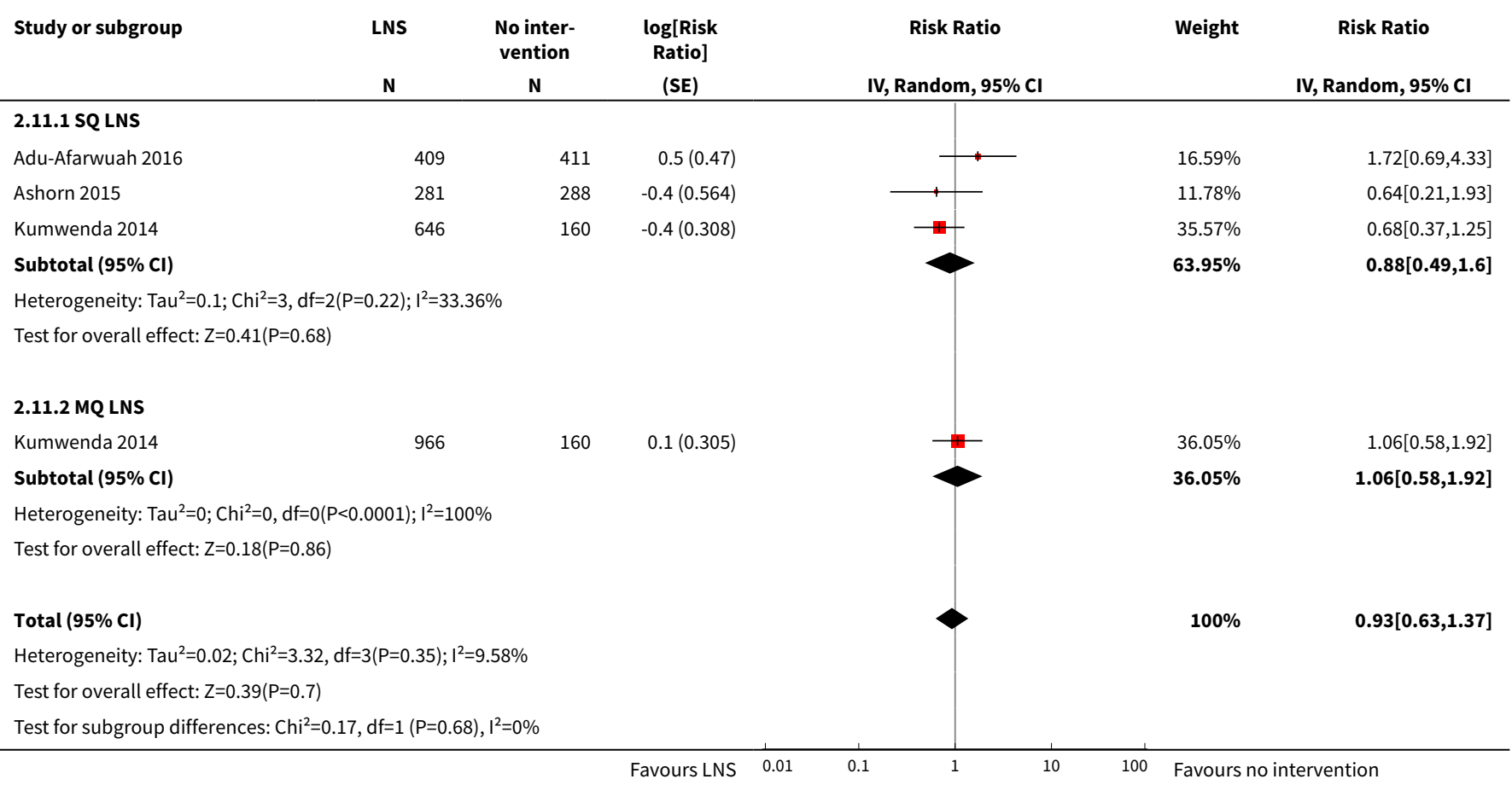

Preventive lipid-based nutrient supplements given with complementary foods to infants and young children 6 to 23 months of age for 
Analysis 2.12. Comparison 2 LNS versus no intervention: Subgroup analysis by energy content/formulation of product provided, Outcome $12 \mathrm{HAZ}$.

\begin{tabular}{|c|c|c|c|c|c|c|}
\hline Study or subgroup & $\begin{array}{l}\text { LNS } \\
\mathrm{N} \\
\end{array}$ & $\begin{array}{c}\text { No inter- } \\
\text { vention } \\
\mathbf{N} \\
\end{array}$ & $\begin{array}{c}\text { Std. Mean } \\
\text { Difference } \\
\text { (SE) } \\
\end{array}$ & $\begin{array}{l}\text { Std. Mean Difference } \\
\text { IV, Random, } 95 \% \mathrm{CI}\end{array}$ & Weight & $\begin{array}{l}\text { Std. Mean Difference } \\
\text { IV, Random, } 95 \% \mathrm{CI}\end{array}$ \\
\hline \multicolumn{7}{|l|}{ 2.12.1 SQ LNS } \\
\hline Adu-Afarwuah 2007 & 97 & 81 & $0.3(0.151)$ & 1 & $2.67 \%$ & $0.26[-0.03,0.55]$ \\
\hline Adu-Afarwuah 2016 & 347 & 350 & $0.2(0.076)$ & $\longrightarrow$ & $6 \%$ & $0.18[0.03,0.33]$ \\
\hline Ashorn 2015 & 214 & 220 & $-0.1(0.098)$ & 1 & $4.68 \%$ & $-0.06[-0.25,0.13]$ \\
\hline Hess 2015 & 2435 & 785 & $0.3(0.047)$ & + & $8.12 \%$ & $0.3[0.21,0.39]$ \\
\hline Iannotti 2014 & 150 & 144 & $-0(0.14)$ & 1 & $2.97 \%$ & $-0.02[-0.29,0.25]$ \\
\hline Kumwenda 2014 & 233 & 60 & $0(0.07)$ & + & $6.4 \%$ & $0.03[-0.11,0.17]$ \\
\hline Kumwenda 2014 & 242 & 60 & $0(0.068)$ & + & $6.54 \%$ & $0.01[-0.12,0.14]$ \\
\hline Luby 2018 & 567 & 1103 & $0.3(0.054)$ & $\rightarrow$ & $7.62 \%$ & $0.26[0.15,0.37]$ \\
\hline Null 2018 & 695 & 1535 & $0.1(0.051)$ & + & $7.84 \%$ & $0.1[0,0.2]$ \\
\hline \multicolumn{7}{|c|}{ Heterogeneity: $\mathrm{Tau}^{2}=0.01 ; \mathrm{Chi}^{2}=31.92, \mathrm{df}=9(\mathrm{P}=0) ; \mathrm{I}^{2}=71.81 \%$} \\
\hline \multicolumn{7}{|c|}{ Test for overall effect: $Z=2.94(P=0)$} \\
\hline \multicolumn{7}{|l|}{ 2.12.2 MQ LNS } \\
\hline Christian 2015 & 1344 & 1265 & $0.1(0.039)$ & + & $8.69 \%$ & $0.11[0.03,0.19]$ \\
\hline Huybregts 2012 & 598 & 440 & $0.3(0.089)$ & $千$ & $5.16 \%$ & $0.27[0.1,0.44]$ \\
\hline Kumwenda 2014 & 237 & 60 & $0(0.073)$ & + & $6.23 \%$ & $0.03[-0.11,0.17]$ \\
\hline Kumwenda 2014 & 241 & 60 & $-0(0.073)$ & + & $6.2 \%$ & $-0.03[-0.17,0.11]$ \\
\hline Mangani 2015 & 212 & 104 & $0(0.072)$ & + & $6.24 \%$ & $0.03[-0.11,0.17]$ \\
\hline Mangani 2015 & 210 & 105 & $0.1(0.068)$ & + & $6.52 \%$ & $0.08[-0.05,0.21]$ \\
\hline Subtotal $(95 \% \mathrm{Cl})$ & & & & 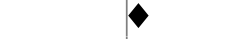 & $39.05 \%$ & $0.08[0.01,0.15]$ \\
\hline \multicolumn{7}{|c|}{ Heterogeneity: $\mathrm{Tau}^{2}=0 ; \mathrm{Chi}^{2}=8.34, \mathrm{df}=5(\mathrm{P}=0.14) ; \mathrm{I}^{2}=40.05 \%$} \\
\hline \multicolumn{7}{|c|}{ Test for overall effect: $Z=2.25(P=0.02)$} \\
\hline \multicolumn{7}{|c|}{ Heterogeneity: $\mathrm{Tau}^{2}=0.01 ; \mathrm{Chi}^{2}=43.43, \mathrm{df}=15(\mathrm{P}=0) ; \mathrm{I}^{2}=65.46 \%$} \\
\hline \multicolumn{7}{|c|}{ Test for overall effect: $\mathrm{Z}=3.69(\mathrm{P}=0)$} \\
\hline Test for subgroup dif & $\mathrm{df}=1(\mathrm{P}=0.4$ & $1^{2}=0 \%$ & & & & \\
\hline
\end{tabular}

\section{Analysis 2.13. Comparison 2 LNS versus no intervention: Subgroup analysis by energy content/formulation of product provided, Outcome $13 \mathrm{WHZ}$.}

\begin{tabular}{lrrr|r} 
Study or subgroup & LNS & $\begin{array}{c}\text { No inter- } \\
\text { vention }\end{array}$ & $\begin{array}{c}\text { Std. Mean } \\
\text { Difference } \\
\text { (SE) }\end{array}$ & Std. Mean Difference \\
IV, Random, 95\% CI
\end{tabular}

Preventive lipid-based nutrient supplements given with complementary foods to infants and young children 6 to 23 months of age for 95 health, nutrition, and developmental outcomes (Review)

Copyright ( 2019 The Cochrane Collaboration. Published by John Wiley \& Sons, Ltd. 


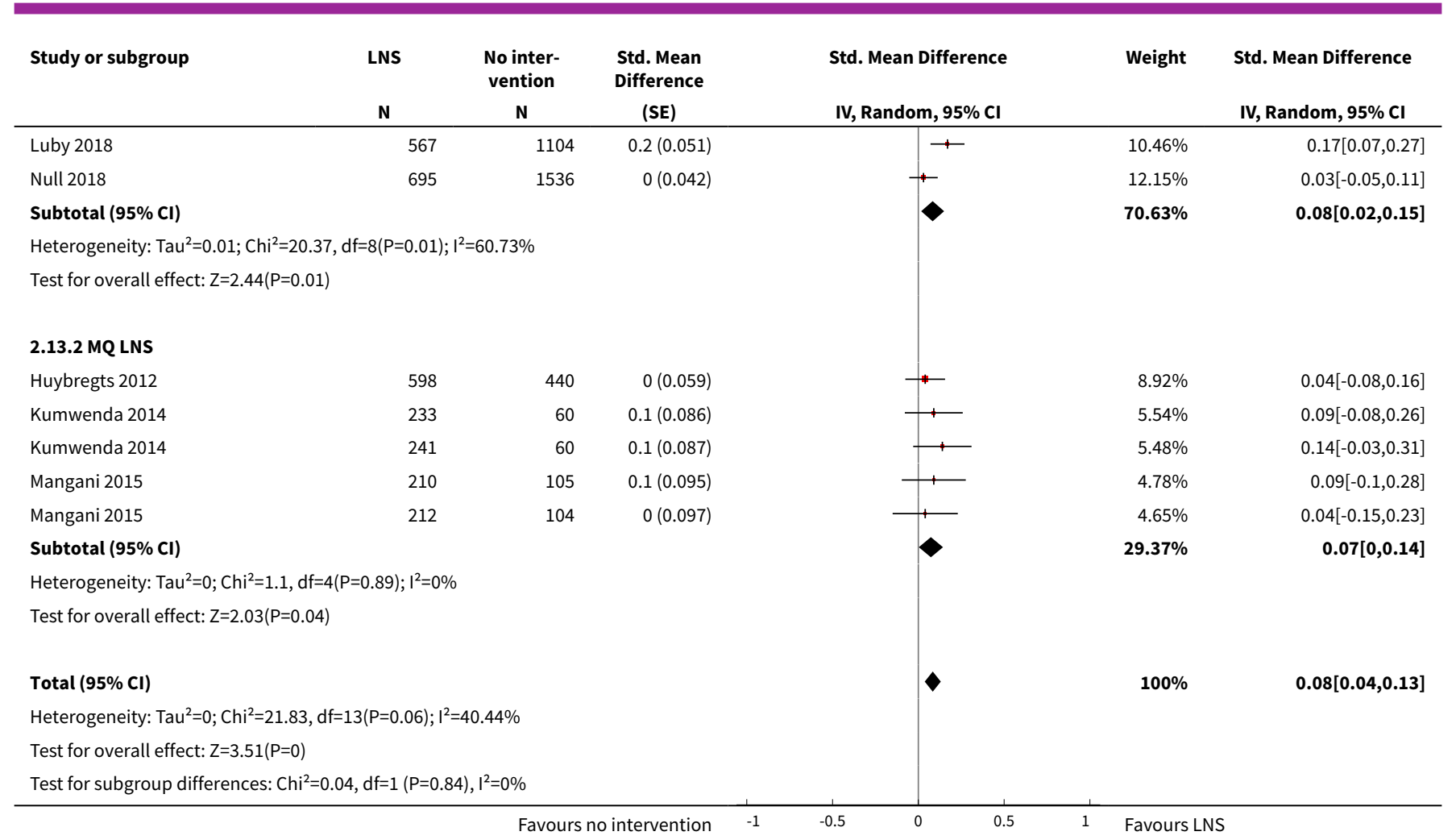

\section{Analysis 2.14. Comparison 2 LNS versus no intervention: Subgroup analysis by energy content/formulation of product provided, Outcome 14 WAZ.}

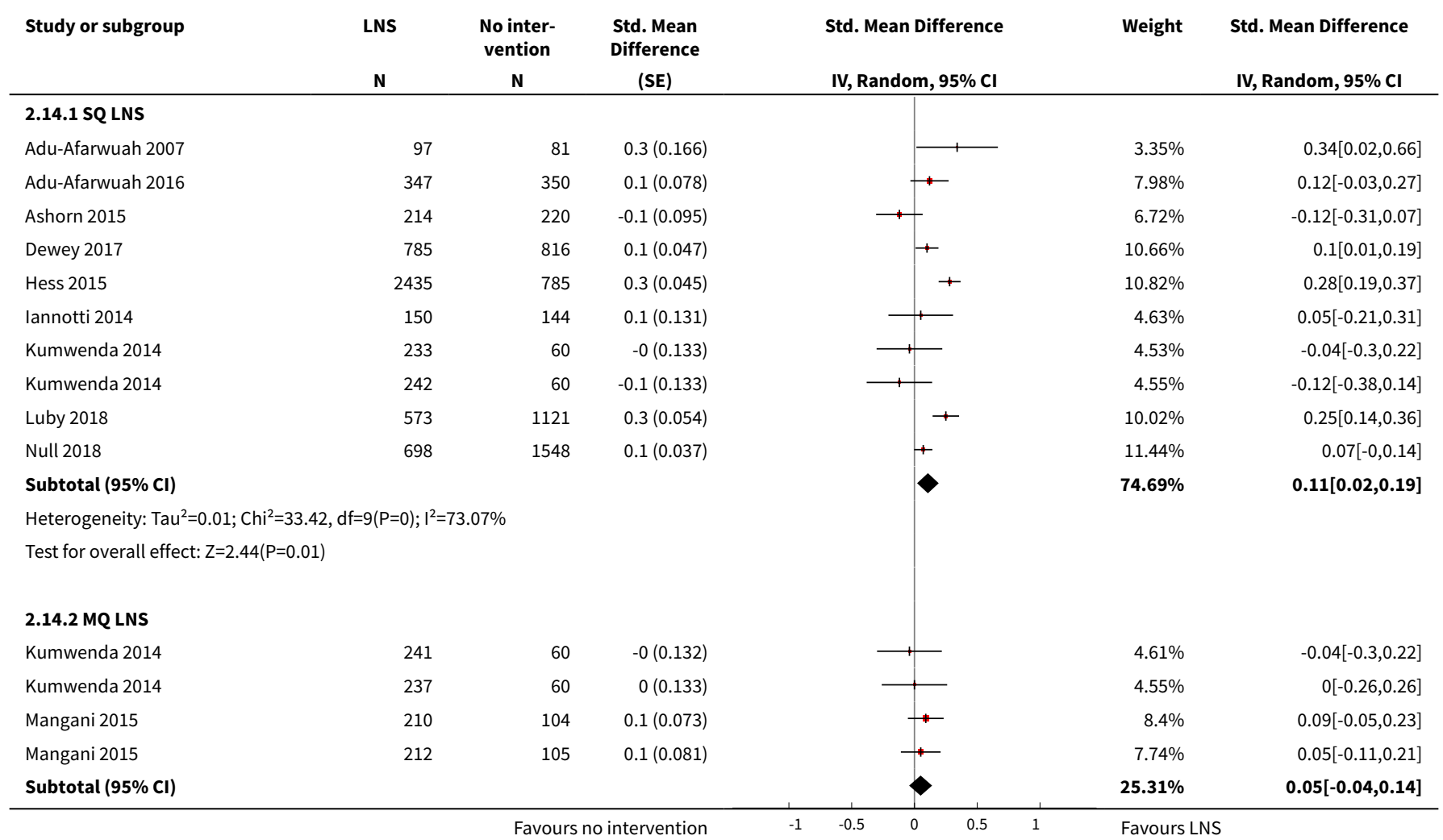




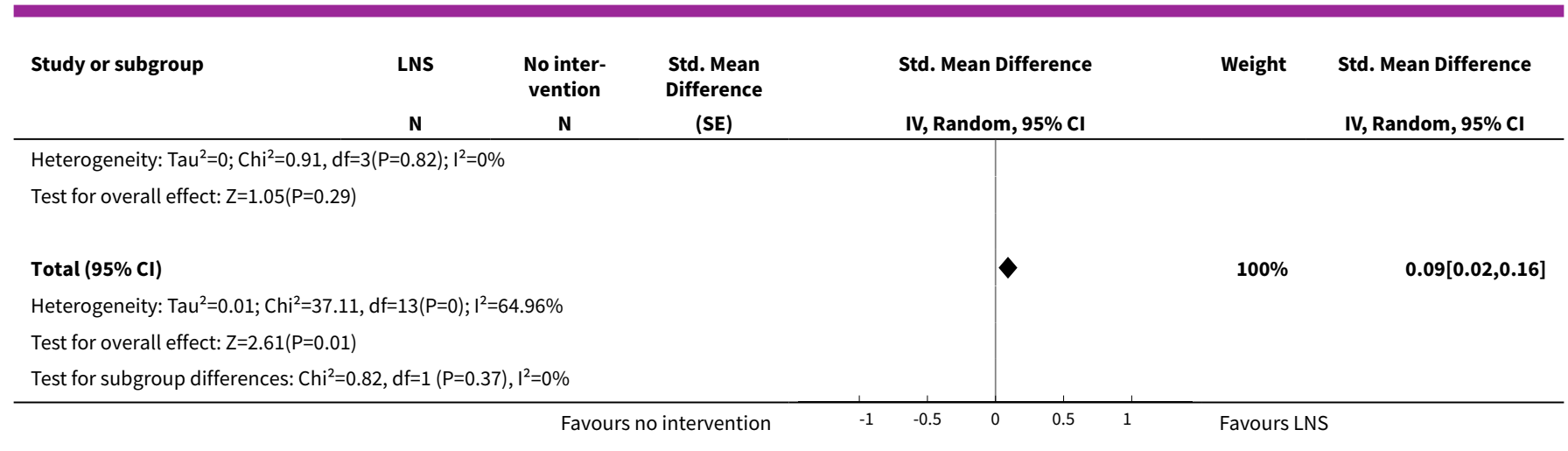

\section{Comparison 3. LNS versus no intervention: Subgroup analysis by duration of intervention}

\begin{tabular}{|c|c|c|c|c|}
\hline $\begin{array}{l}\text { Outcome or subgroup } \\
\text { title }\end{array}$ & No. of studies & $\begin{array}{l}\text { No. of partici- } \\
\text { pants }\end{array}$ & Statistical method & Effect size \\
\hline 1 Moderate stunting & 9 & 13372 & Risk Ratio (Random, 95\% Cl) & $0.93[0.88,0.98]$ \\
\hline 1.16 to 12 months & 6 & 7871 & Risk Ratio (Random, 95\% Cl) & $0.96[0.89,1.03]$ \\
\hline $\begin{array}{l}1.2 \text { More than } 12 \\
\text { months }\end{array}$ & 3 & 5501 & Risk Ratio (Random, 95\% Cl) & $0.89[0.81,0.97]$ \\
\hline 2 Severe stunting & 5 & 6151 & Risk Ratio (Random, 95\% Cl) & $0.85[0.74,0.98]$ \\
\hline 2.16 to 12 months & 3 & 2251 & Risk Ratio (Random, 95\% Cl) & $0.89[0.74,1.07]$ \\
\hline $\begin{array}{l}2.2 \text { More than } 12 \\
\text { months }\end{array}$ & 2 & 3900 & Risk Ratio (Random, 95\% Cl) & $0.80[0.64,0.99]$ \\
\hline 3 Moderate wasting & 8 & 13172 & Risk Ratio (Random, 95\% Cl) & $0.82[0.74,0.91]$ \\
\hline 3.16 to 12 months & 5 & 7669 & Risk Ratio (Random, 95\% Cl) & $0.81[0.72,0.92]$ \\
\hline $\begin{array}{l}3.2 \text { More than } 12 \\
\text { months }\end{array}$ & 3 & 5503 & Risk Ratio (Random, 95\% Cl) & $0.83[0.69,0.99]$ \\
\hline $\begin{array}{l}4 \text { Severe wasting: } 6 \text { to } \\
12 \text { months }\end{array}$ & 3 & 2329 & Risk Ratio (Random, 95\% Cl) & $1.27[0.66,2.46]$ \\
\hline $\begin{array}{l}5 \text { Moderate under- } \\
\text { weight }\end{array}$ & 8 & 13073 & Risk Ratio (Random, 95\% Cl) & $0.85[0.80,0.91]$ \\
\hline 5.16 to 12 months & 5 & 7532 & Risk Ratio (Random, 95\% Cl) & $0.87[0.78,0.97]$ \\
\hline $\begin{array}{l}5.2 \text { More than } 12 \\
\text { months }\end{array}$ & 3 & 5541 & Risk Ratio (Random, 95\% Cl) & $0.87[0.79,0.95]$ \\
\hline $\begin{array}{l}6 \text { Severe underweight: } 6 \\
\text { to } 12 \text { months }\end{array}$ & 2 & 1729 & Risk Ratio (Random, 95\% Cl) & $0.78[0.54,1.13]$ \\
\hline 7 Anaemia & 5 & 2332 & Risk Ratio (M-H, Random, 95\% Cl) & $0.79[0.69,0.90]$ \\
\hline 7.1 Upto 6 months & 2 & 1176 & Risk Ratio (M-H, Random, 95\% Cl) & $0.55[0.20,1.47]$ \\
\hline
\end{tabular}

Preventive lipid-based nutrient supplements given with complementary foods to infants and young children 6 to 23 months of age for 


\begin{tabular}{|c|c|c|c|c|}
\hline $\begin{array}{l}\text { Outcome or subgroup } \\
\text { title }\end{array}$ & No. of studies & $\begin{array}{l}\text { No. of partici- } \\
\text { pants }\end{array}$ & Statistical method & Effect size \\
\hline 7.26 to 12 months & 2 & 627 & Risk Ratio (M-H, Random, 95\% Cl) & $0.84[0.71,1.00]$ \\
\hline $\begin{array}{l}7.3 \text { More than } 12 \\
\text { months }\end{array}$ & 1 & 529 & Risk Ratio (M-H, Random, 95\% Cl) & $0.70[0.55,0.88]$ \\
\hline 8 Adverse effects & 3 & 3382 & Risk Ratio (Random, 95\% Cl) & $0.86[0.74,1.01]$ \\
\hline $\begin{array}{l}9 \text { Mid-upper arm cir- } \\
\text { cumference (MUAC) }\end{array}$ & 6 & 8187 & Std. Mean Difference (Random, 95\% Cl) & $0.13[0.05,0.22]$ \\
\hline 9.1 Upto 6 months & 2 & 1472 & Std. Mean Difference (Random, 95\% Cl) & $0.06[-0.23,0.35]$ \\
\hline 9.26 to 12 months & 3 & 5114 & Std. Mean Difference (Random, 95\% Cl) & $0.18[0.10,0.26]$ \\
\hline $\begin{array}{l}9.3 \text { More than } 12 \\
\text { months }\end{array}$ & 1 & 1601 & Std. Mean Difference (Random, 95\% Cl) & $0.04[-0.04,0.12]$ \\
\hline $\begin{array}{l}10 \text { Serum haemoglobin } \\
(\mathrm{g} / \mathrm{L})\end{array}$ & 4 & 4518 & Mean Difference (IV, Random, 95\% CI) & $5.78[2.27,9.30]$ \\
\hline 10.1 Upto 6 months & 2 & 1176 & Mean Difference (IV, Random, 95\% CI) & $5.58[0.52,10.64]$ \\
\hline 10.26 to 12 months & 2 & 3342 & Mean Difference (IV, Random, 95\% CI) & $5.96[0.08,11.84]$ \\
\hline 11 Mortality & 3 & 3321 & Risk Ratio (Random, 95\% Cl) & $0.93[0.63,1.37]$ \\
\hline 11.16 to 12 months & 3 & 3321 & Risk Ratio (Random, 95\% Cl) & $0.93[0.63,1.37]$ \\
\hline $12 \mathrm{HAZ}$ & 12 & 15795 & Std. Mean Difference (Random, 95\% Cl) & $0.11[0.05,0.16]$ \\
\hline 12.1 Upto 6 months & 3 & 1510 & Std. Mean Difference (Random, 95\% Cl) & $0.19[0.01,0.36]$ \\
\hline 12.26 to 12 months & 6 & 8784 & Std. Mean Difference (Random, 95\% Cl) & $0.08[0.00,0.15]$ \\
\hline $\begin{array}{l}12.3 \text { More than } 12 \\
\text { months }\end{array}$ & 3 & 5501 & Std. Mean Difference (Random, 95\% Cl) & $0.14[0.03,0.25]$ \\
\hline 13 WAZ & 10 & 12188 & Std. Mean Difference (Random, 95\% Cl) & $0.09[0.02,0.16]$ \\
\hline 13.1 Upto 6 months & 3 & 1103 & Std. Mean Difference (Random, 95\% Cl) & $0.09[-0.00,0.19]$ \\
\hline 13.26 to 12 months & 4 & 5544 & Std. Mean Difference (Random, 95\% Cl) & $0.03[-0.12,0.18]$ \\
\hline $\begin{array}{l}13.3 \text { More than } 12 \\
\text { months }\end{array}$ & 3 & 5541 & Std. Mean Difference (Random, 95\% Cl) & $0.13[0.03,0.24]$ \\
\hline $14 \mathrm{WHZ}$ & 10 & 12894 & Std. Mean Difference (Random, 95\% Cl) & $0.08[0.04,0.13]$ \\
\hline 14.1 Upto 6 months & 2 & 1216 & Std. Mean Difference (Random, 95\% Cl) & $0.13[-0.12,0.38]$ \\
\hline 14.26 to 12 months & 5 & 6175 & Std. Mean Difference (Random, 95\% Cl) & $0.07[0.00,0.14]$ \\
\hline $\begin{array}{l}14.3 \text { More than } 12 \\
\text { months }\end{array}$ & 3 & 5503 & Std. Mean Difference (Random, 95\% Cl) & $0.09[0.01,0.17]$ \\
\hline
\end{tabular}


Analysis 3.1. Comparison 3 LNS versus no intervention: Subgroup analysis by duration of intervention, Outcome 1 Moderate stunting.

\begin{tabular}{|c|c|c|c|c|c|c|}
\hline Study or subgroup & LNS & $\begin{array}{c}\text { No inter- } \\
\text { vention } \\
\mathbf{N} \\
\end{array}$ & $\begin{array}{c}\text { log[Risk } \\
\text { Ratio] } \\
\text { (SE) }\end{array}$ & $\begin{array}{c}\text { Risk Ratio } \\
\text { IV, Random, } 95 \% \text { CI } \\
\end{array}$ & Weight & $\begin{array}{c}\text { Risk Ratio } \\
\text { IV, Random, } 95 \% \mathrm{CI} \\
\end{array}$ \\
\hline \multicolumn{7}{|l|}{ 3.1.1 6 to 12 months } \\
\hline Adu-Afarwuah 2016 & 347 & 350 & $-0.4(0.218)$ & 1 & $1.74 \%$ & $0.65[0.43,1]$ \\
\hline Ashorn 2015 & 214 & 220 & $0.1(0.13)$ & 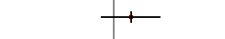 & $4.44 \%$ & $1.16[0.9,1.49]$ \\
\hline Christian 2015 & 1344 & 1265 & $-0.1(0.046)$ & $*$ & $18.83 \%$ & $0.91[0.83,1]$ \\
\hline Kumwenda 2014 & 207 & 43 & $-0.1(0.163)$ & $\longrightarrow$ & $2.98 \%$ & $0.93[0.68,1.29]$ \\
\hline Kumwenda 2014 & 184 & 43 & $0(0.163)$ & 1 & $2.98 \%$ & $1.01[0.74,1.4]$ \\
\hline Kumwenda 2014 & 187 & 43 & $0.1(0.155)$ & + & $3.25 \%$ & $1.16[0.86,1.57]$ \\
\hline Kumwenda 2014 & 195 & 44 & $0.1(0.157)$ & -1 & $3.2 \%$ & $1.1[0.81,1.49]$ \\
\hline Mangani 2015 & 191 & 93 & $-0.1(0.12)$ & 1 & $5.14 \%$ & $0.94[0.75,1.19]$ \\
\hline Mangani 2015 & 188 & 92 & $0.1(0.113)$ & 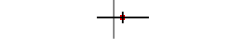 & $5.64 \%$ & $1.08[0.87,1.35]$ \\
\hline \multicolumn{7}{|c|}{ Heterogeneity: $\mathrm{Tau}^{2}=0 ; \mathrm{Chi}^{2}=11.43, \mathrm{df}=9(\mathrm{P}=0.25) ; \mathrm{I}^{2}=21.29 \%$} \\
\hline \multicolumn{7}{|c|}{ Test for overall effect: $Z=1.14(P=0.25)$} \\
\hline \multicolumn{7}{|c|}{ 3.1.2 More than 12 months } \\
\hline Dewey 2017 & 785 & 816 & $-0.1(0.058)$ & * & $14.77 \%$ & $0.93[0.83,1.04]$ \\
\hline Luby 2018 & 567 & 1103 & $-0.2(0.07)$ & + & $11.66 \%$ & $0.8[0.7,0.92]$ \\
\hline Null 2018 & 695 & 1535 & $-0.1(0.07)$ & $\rightarrow$ & $11.61 \%$ & $0.92[0.8,1.06]$ \\
\hline Subtotal $(95 \% \mathrm{Cl})$ & & & & 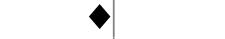 & $38.04 \%$ & $0.89[0.81,0.97]$ \\
\hline \multicolumn{7}{|c|}{ Heterogeneity: $\mathrm{Tau}^{2}=0 ; \mathrm{Chi}^{2}=3.03, \mathrm{df}=2(\mathrm{P}=0.22) ; \mathrm{I}^{2}=34.03 \%$} \\
\hline \multicolumn{7}{|c|}{ Test for overall effect: $Z=2.57(P=0.01)$} \\
\hline Total $(95 \% \mathrm{Cl})$ & & & & 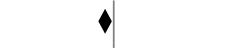 & $100 \%$ & $0.93[0.88,0.98]$ \\
\hline \multicolumn{7}{|c|}{ Heterogeneity: $\mathrm{Tau}^{2}=0 ; \mathrm{Chi}^{2}=15.9, \mathrm{df}=12(\mathrm{P}=0.2) ; \mathrm{I}^{2}=24.54 \%$} \\
\hline
\end{tabular}

Analysis 3.2. Comparison 3 LNS versus no intervention: Subgroup analysis by duration of intervention, Outcome 2 Severe stunting.

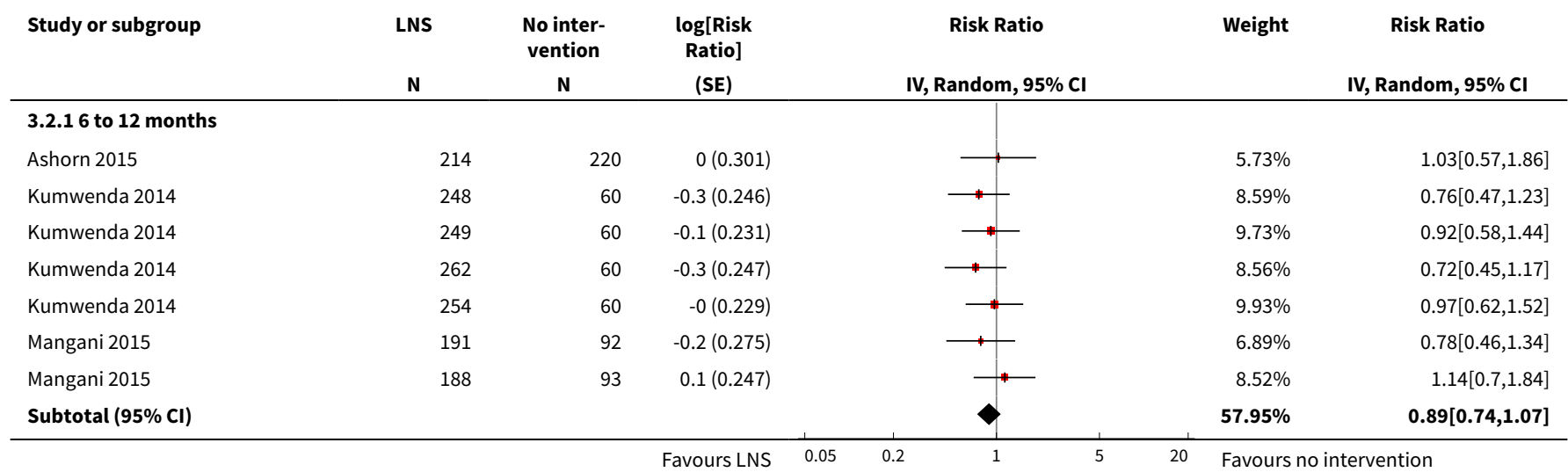

Preventive lipid-based nutrient supplements given with complementary foods to infants and young children 6 to 23 months of age for 99 health, nutrition, and developmental outcomes (Review)

Copyright (c) 2019 The Cochrane Collaboration. Published by John Wiley \& Sons, Ltd. 


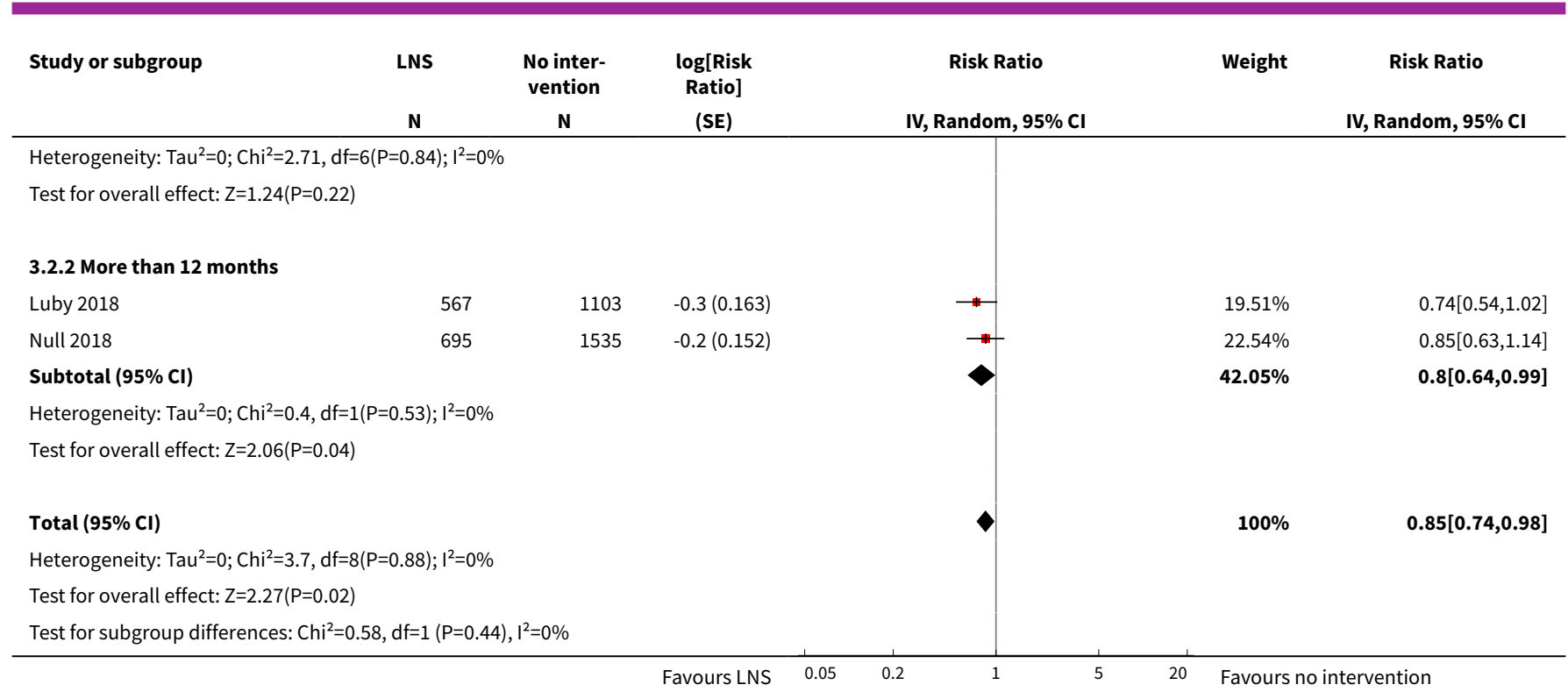

Analysis 3.3. Comparison 3 LNS versus no intervention: Subgroup analysis by duration of intervention, Outcome 3 Moderate wasting.

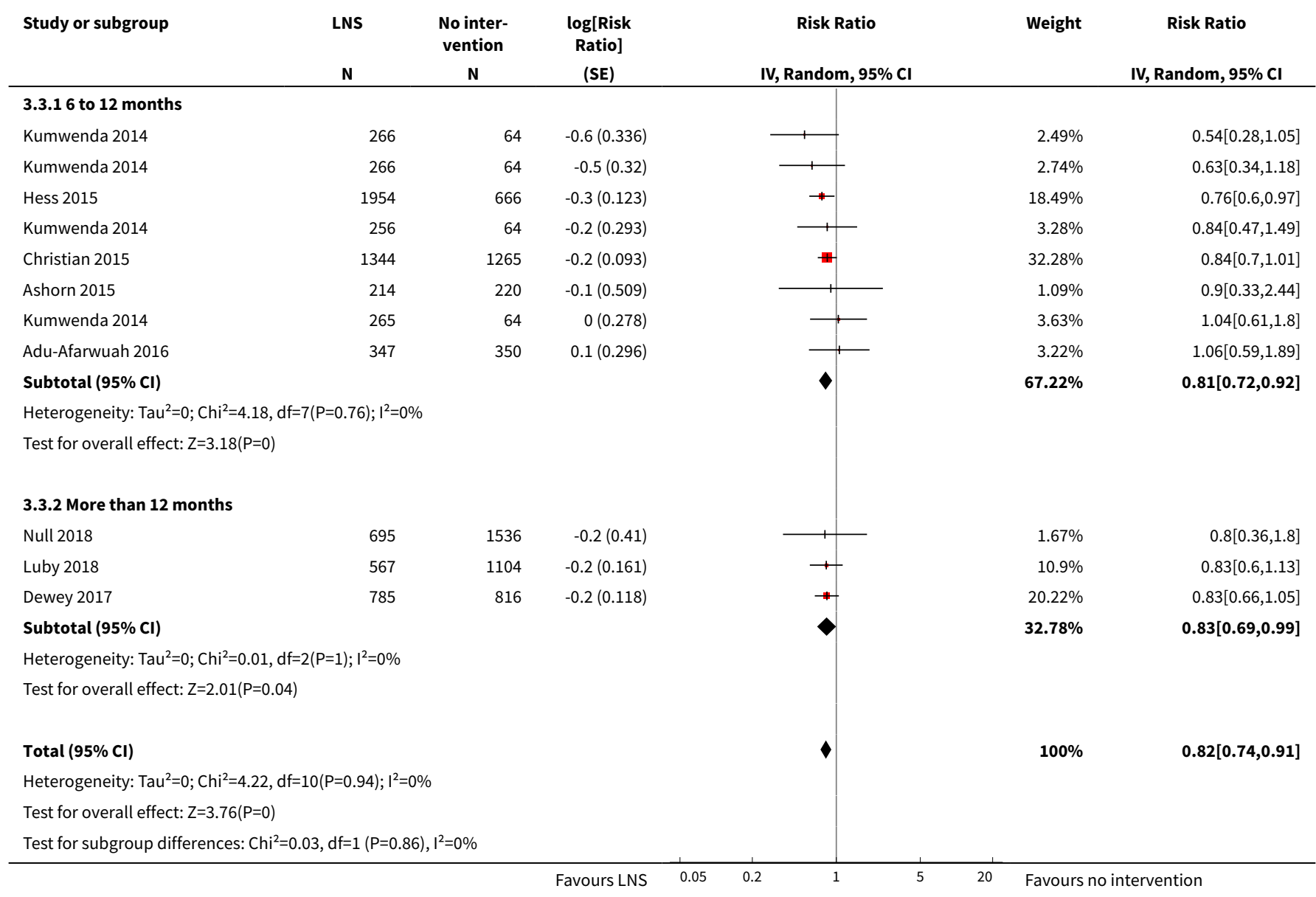

Preventive lipid-based nutrient supplements given with complementary foods to infants and young children 6 to 23 months of age for 


\section{Analysis 3.4. Comparison 3 LNS versus no intervention: Subgroup analysis} by duration of intervention, Outcome 4 Severe wasting: 6 to 12 months.

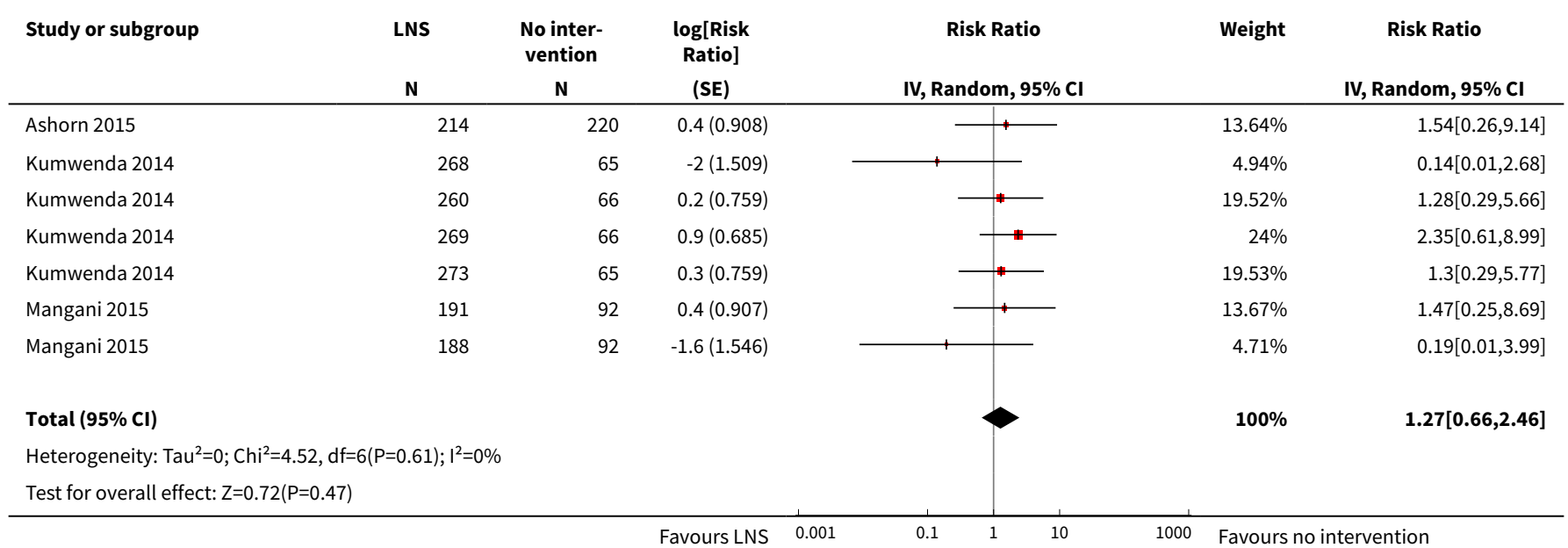

Analysis 3.5. Comparison 3 LNS versus no intervention: Subgroup analysis by duration of intervention, Outcome 5 Moderate underweight.

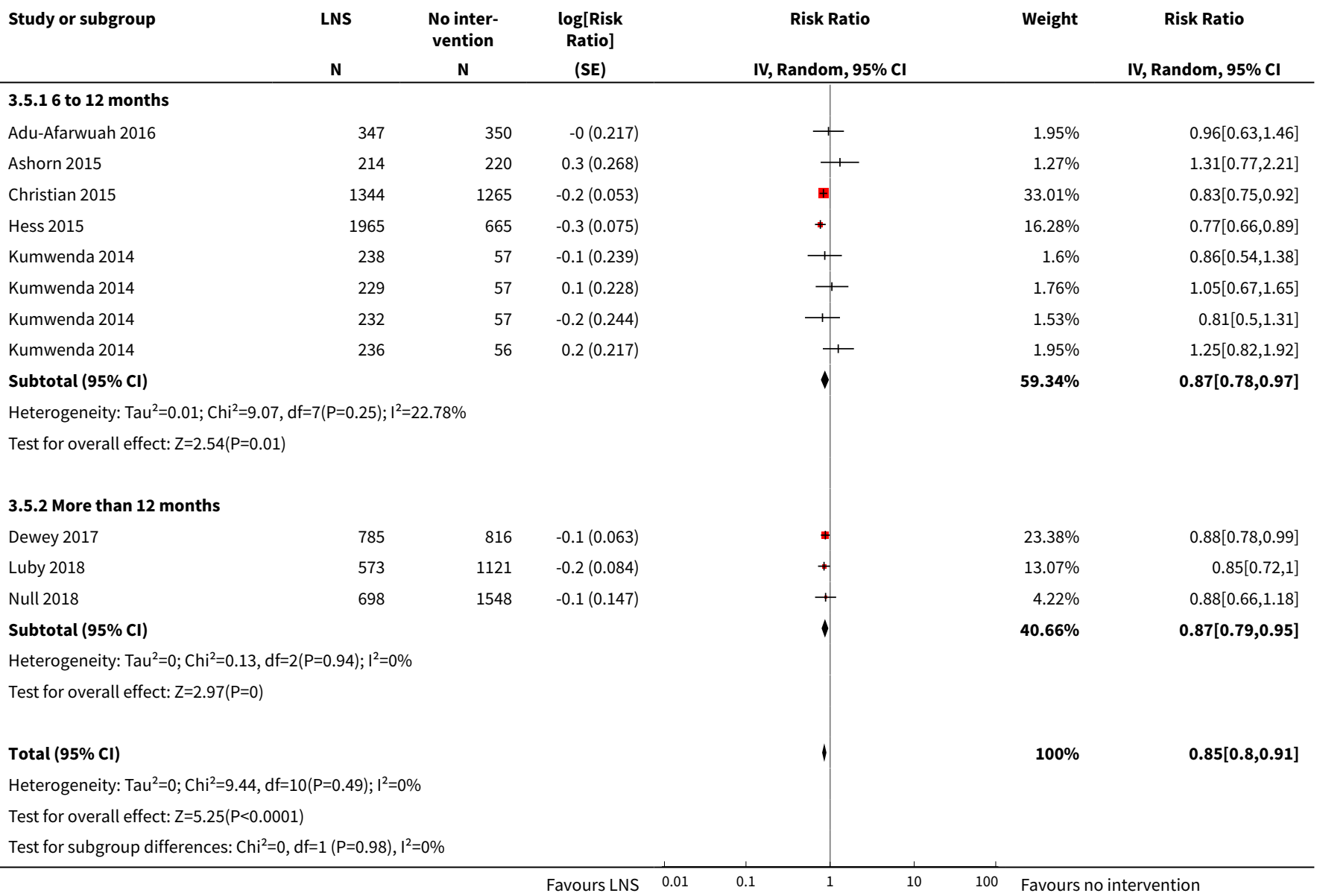

Preventive lipid-based nutrient supplements given with complementary foods to infants and young children 6 to 23 months of age for 
Analysis 3.6. Comparison 3 LNS versus no intervention: Subgroup analysis by duration of intervention, Outcome 6 Severe underweight: 6 to 12 months.

\begin{tabular}{|c|c|c|c|c|c|c|}
\hline Study or subgroup & $\begin{array}{l}\text { LNS } \\
\mathbf{N} \\
\end{array}$ & $\begin{array}{c}\text { No inter- } \\
\text { vention } \\
\mathbf{N} \\
\end{array}$ & $\begin{array}{c}\text { log[Risk } \\
\text { Ratio] } \\
\text { (SE) }\end{array}$ & $\begin{array}{c}\text { Risk Ratio } \\
\text { IV, Random, } 95 \% \mathrm{CI} \\
\end{array}$ & Weight & $\begin{array}{c}\text { Risk Ratio } \\
\text { IV, Random, } 95 \% \mathrm{CI}\end{array}$ \\
\hline Ashorn 2015 & 214 & 220 & $-0.4(0.638)$ & $\longrightarrow$ & $8.51 \%$ & $0.69[0.2,2.39]$ \\
\hline Kumwenda 2014 & 264 & 62 & $-0.4(0.405)$ & $\rightarrow$ & $21.2 \%$ & $0.69[0.31,1.52]$ \\
\hline Kumwenda 2014 & 260 & 63 & $0.1(0.361)$ & 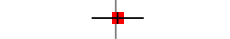 & $26.65 \%$ & $1.05[0.52,2.14]$ \\
\hline Kumwenda 2014 & 265 & 63 & $-0.3(0.393)$ & $\rightarrow$ & $22.45 \%$ & $0.75[0.35,1.61]$ \\
\hline Total $(95 \% \mathrm{Cl})$ & & & & & $100 \%$ & $0.78[0.54,1.13]$ \\
\hline \multicolumn{7}{|c|}{ Heterogeneity: $\mathrm{Tau}^{2}=0 ; \mathrm{Chi}^{2}=0.97, \mathrm{df}=4(\mathrm{P}=0.91) ; \mathrm{I}^{2}=0 \%$} \\
\hline Test for overall effect & & & & & & \\
\hline
\end{tabular}

\section{Analysis 3.7. Comparison 3 LNS versus no intervention: Subgroup analysis by duration of intervention, Outcome 7 Anaemia.}

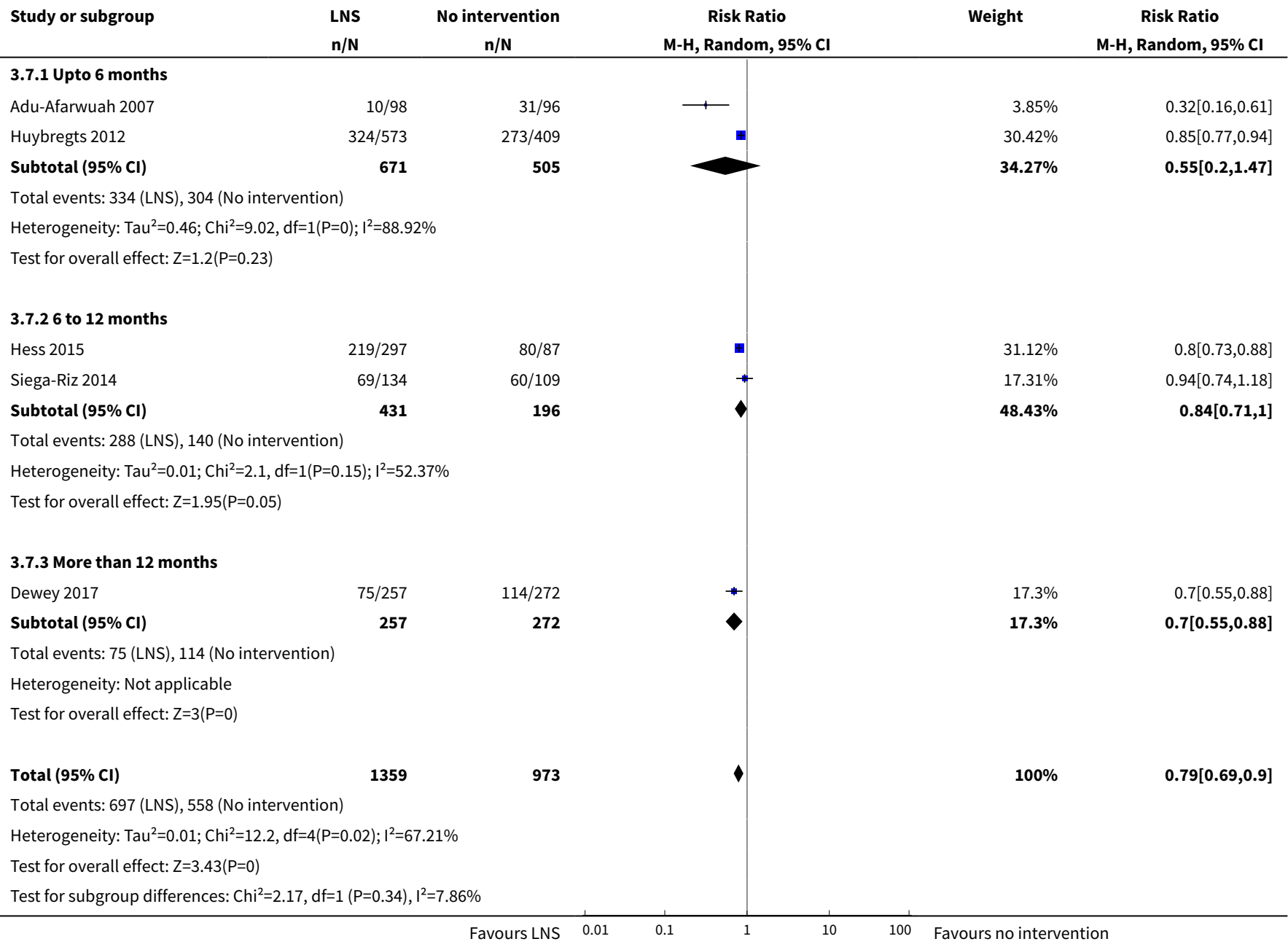

Preventive lipid-based nutrient supplements given with complementary foods to infants and young children 6 to 23 months of age for 
Analysis 3.8. Comparison 3 LNS versus no intervention: Subgroup analysis by duration of intervention, Outcome 8 Adverse effects.

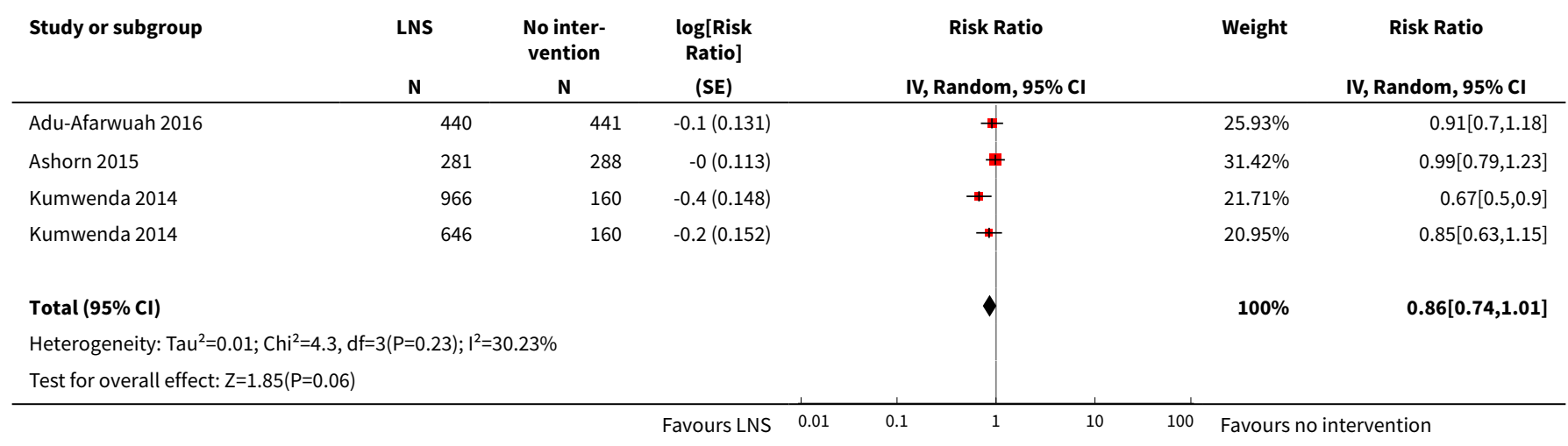

\section{Analysis 3.9. Comparison 3 LNS versus no intervention: Subgroup analysis by duration of intervention, Outcome 9 Mid-upper arm circumference (MUAC).}

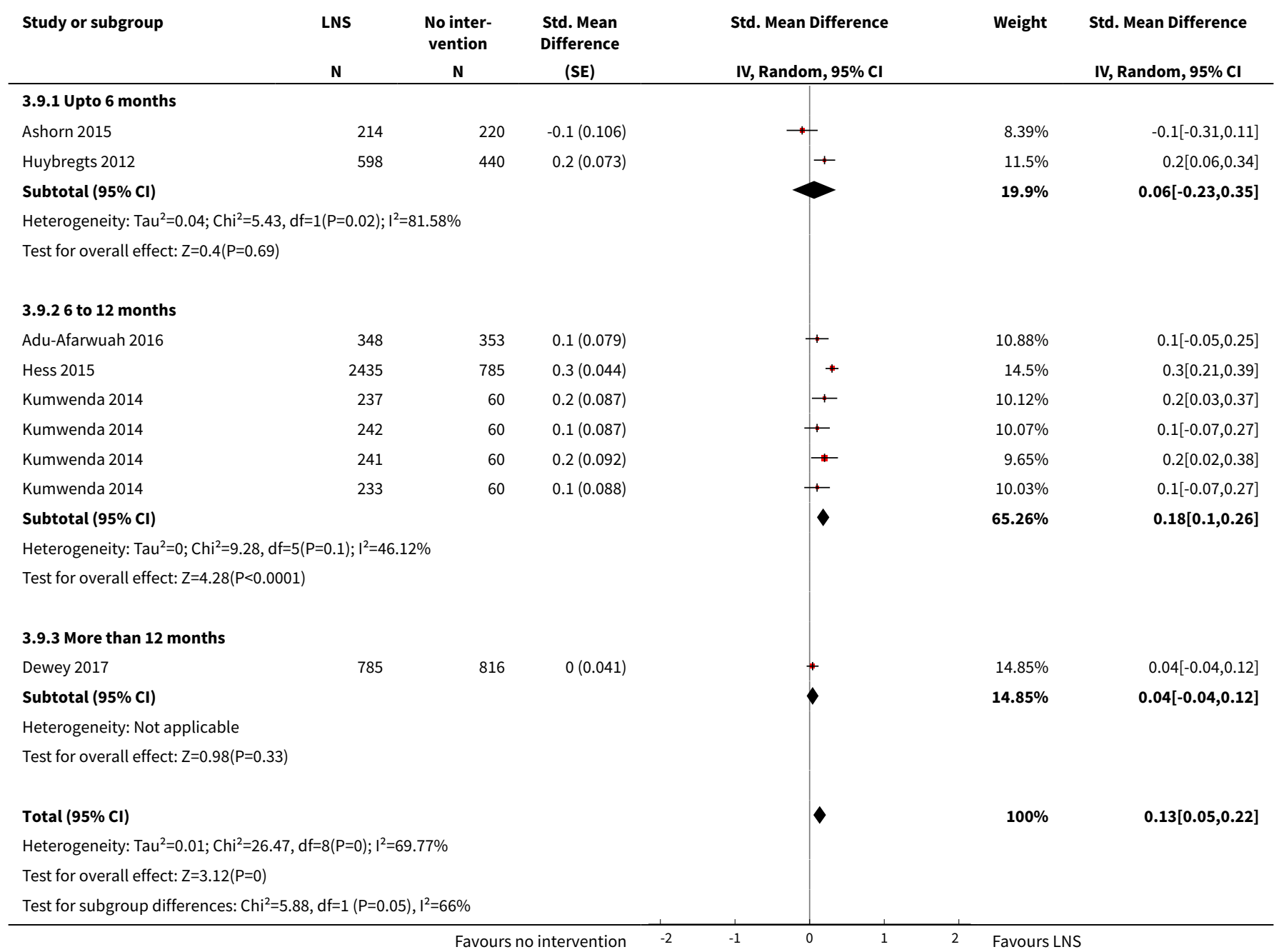

Preventive lipid-based nutrient supplements given with complementary foods to infants and young children 6 to 23 months of age for 
Analysis 3.10. Comparison 3 LNS versus no intervention: Subgroup analysis by duration of intervention, Outcome 10 Serum haemoglobin (g/L).

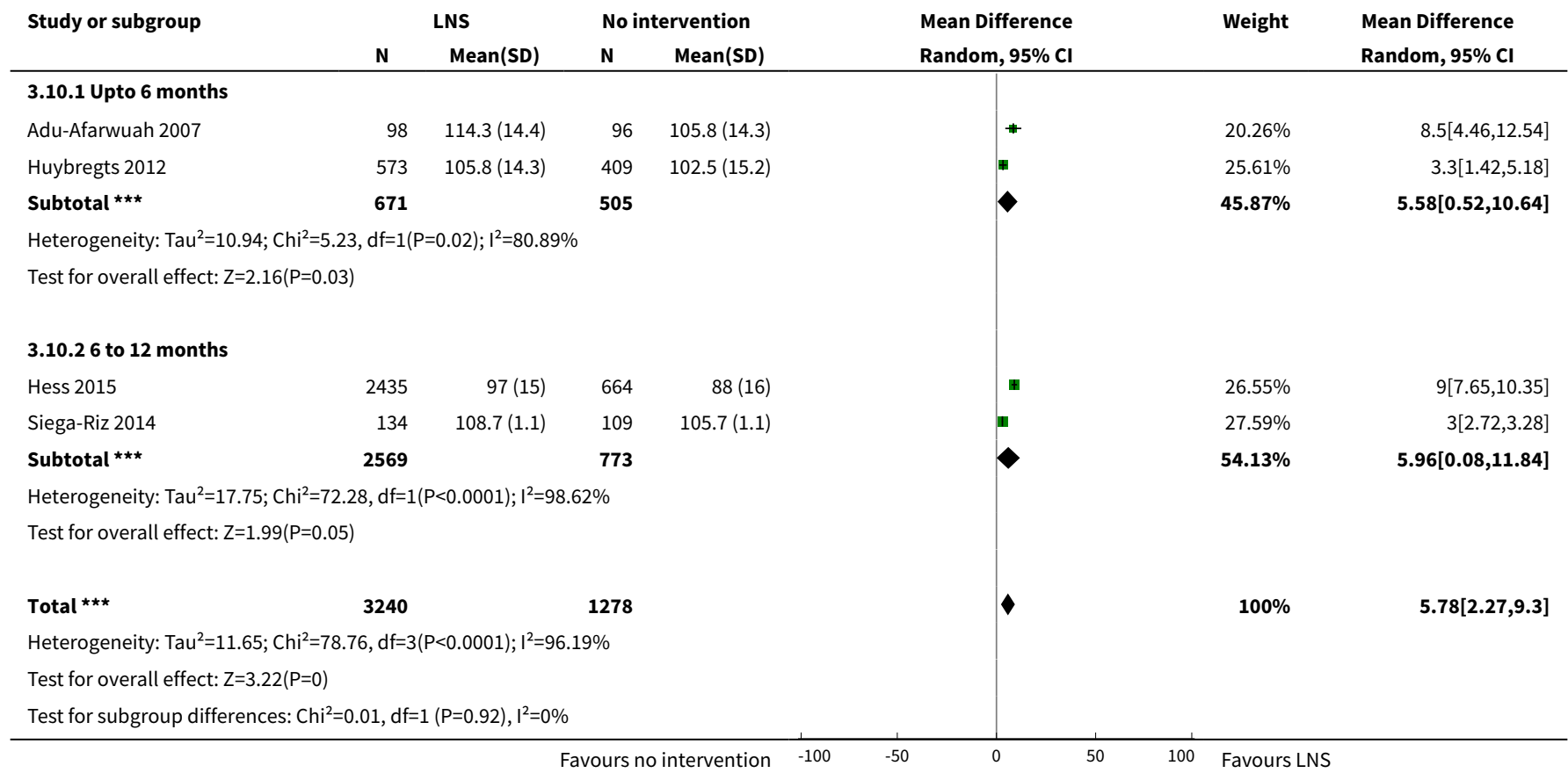

Analysis 3.11. Comparison 3 LNS versus no intervention: Subgroup analysis by duration of intervention, Outcome 11 Mortality.

\begin{tabular}{|c|c|c|c|}
\hline Study or subgroup & LNS & $\begin{array}{l}\text { No inter- } \\
\text { vention }\end{array}$ & $\begin{array}{c}\text { log[Risk } \\
\text { Ratio] } \\
\text { (SE) }\end{array}$ \\
\hline \multicolumn{4}{|l|}{ 3.11.1 6 to 12 months } \\
\hline Adu-Afarwuah 2016 & 409 & 411 & $0.5(0.47)$ \\
\hline Ashorn 2015 & 281 & 288 & $-0.4(0.564)$ \\
\hline Kumwenda 2014 & 966 & 160 & $0.1(0.305)$ \\
\hline Kumwenda 2014 & 646 & 160 & $-0.4(0.308)$ \\
\hline
\end{tabular}

Subtotal $(95 \% \mathrm{Cl})$

Heterogeneity: $\mathrm{Tau}^{2}=0.02 ; \mathrm{Chi}^{2}=3.32, \mathrm{df}=3(\mathrm{P}=0.35) ; \mathrm{I}^{2}=9.58 \%$

Test for overall effect: $\mathrm{Z}=0.39(\mathrm{P}=0.7)$

Total $(95 \% \mathrm{Cl})$

Heterogeneity: $\mathrm{Tau}^{2}=0.02 ; \mathrm{Chi}^{2}=3.32, \mathrm{df}=3(\mathrm{P}=0.35) ; \mathrm{I}^{2}=9.58 \%$

Test for overall effect: $\mathrm{Z}=0.39(\mathrm{P}=0.7)$ 
Analysis 3.12. Comparison 3 LNS versus no intervention:

Subgroup analysis by duration of intervention, Outcome 12 HAZ.

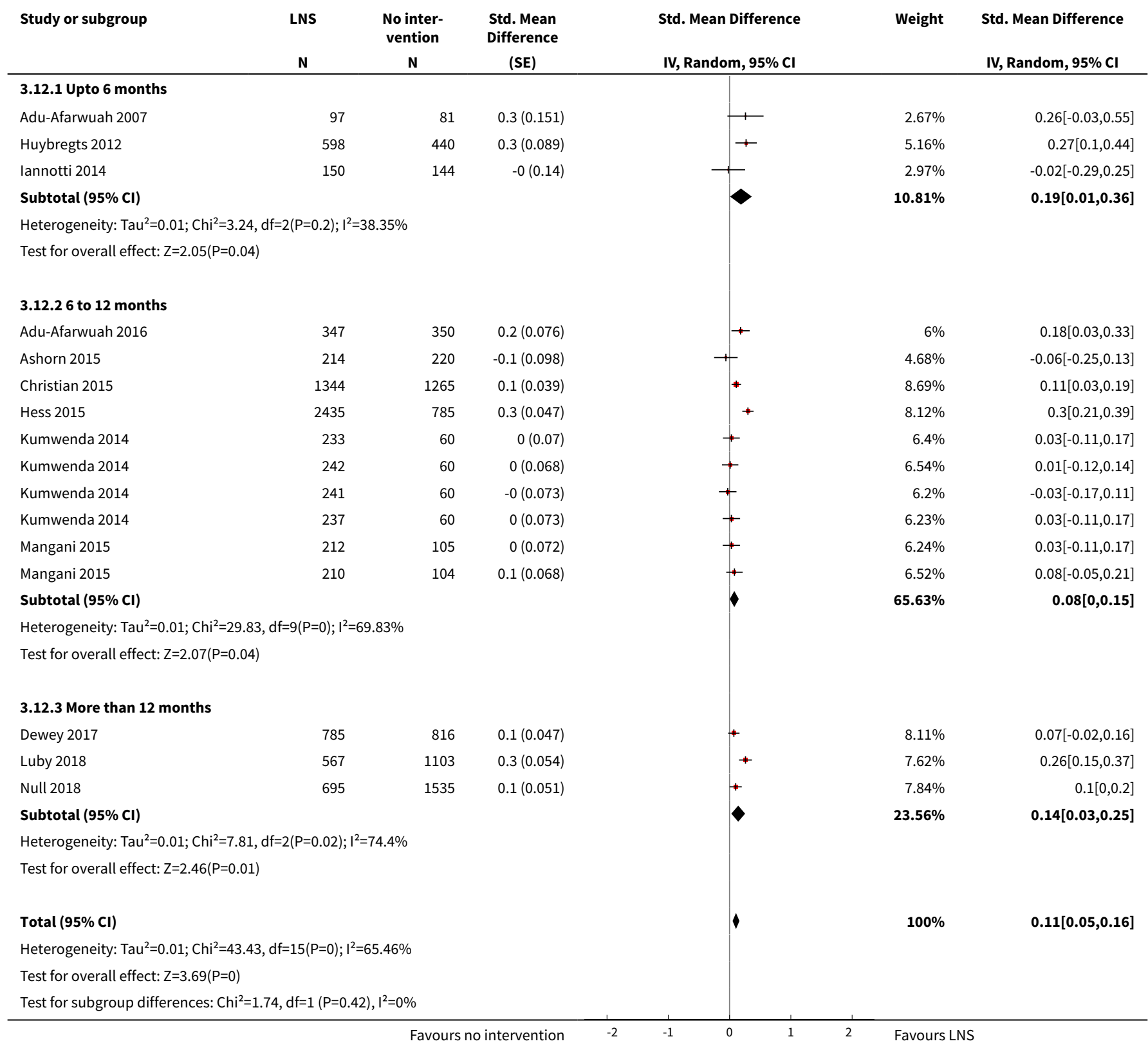

Analysis 3.13. Comparison 3 LNS versus no intervention: Subgroup analysis by duration of intervention, Outcome 13 WAZ.

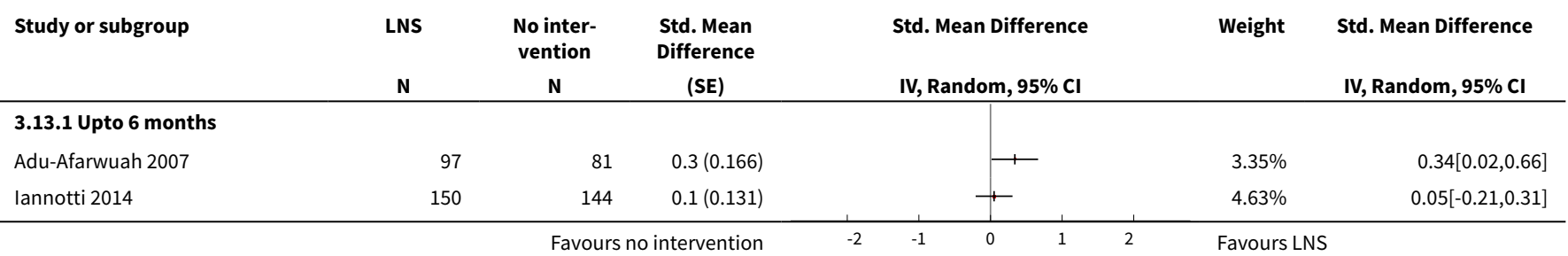

Preventive lipid-based nutrient supplements given with complementary foods to infants and young children 6 to 23 months of age for 105 health, nutrition, and developmental outcomes (Review)

Copyright (c) 2019 The Cochrane Collaboration. Published by John Wiley \& Sons, Ltd. 


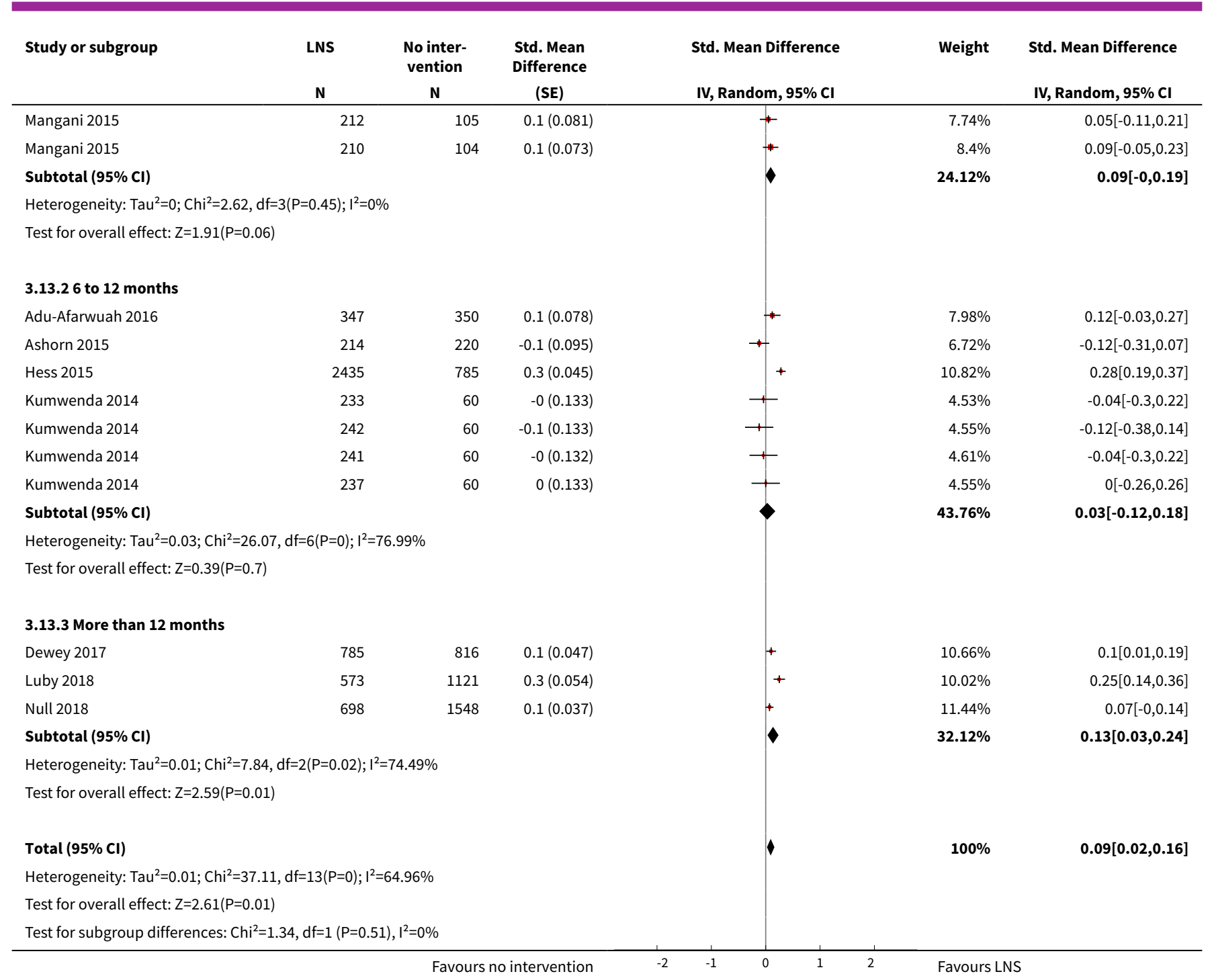

Analysis 3.14. Comparison 3 LNS versus no intervention: Subgroup analysis by duration of intervention, Outcome 14 WHZ.

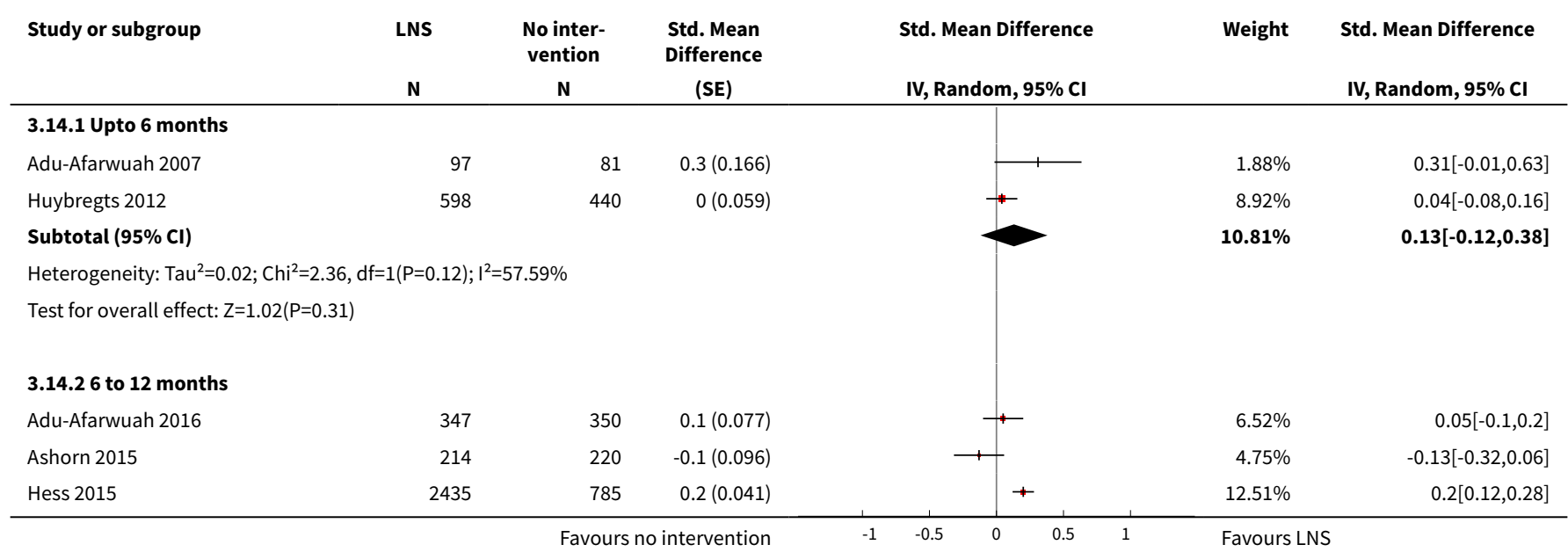




\begin{tabular}{|c|c|c|c|c|c|c|}
\hline Study or subgroup & $\begin{array}{l}\text { LNS } \\
\mathbf{N} \\
\end{array}$ & $\begin{array}{c}\text { No inter- } \\
\text { vention } \\
\mathbf{N}\end{array}$ & $\begin{array}{l}\text { Std. Mean } \\
\text { Difference } \\
\text { (SE) } \\
\end{array}$ & $\begin{array}{l}\text { Std. Mean Difference } \\
\text { IV, Random, } 95 \% \text { CI }\end{array}$ & Weight & $\begin{array}{l}\text { Std. Mean Difference } \\
\text { IV, Random, } 95 \% \mathrm{CI}\end{array}$ \\
\hline Kumwenda 2014 & 241 & 60 & $0.1(0.087)$ & 1 & $5.48 \%$ & $0.14[-0.03,0.31]$ \\
\hline Kumwenda 2014 & 242 & 60 & $-0(0.092)$ & $\longrightarrow$ & $5.03 \%$ & $-0.01[-0.19,0.17]$ \\
\hline Kumwenda 2014 & 233 & 60 & $0.1(0.086)$ & + & $5.54 \%$ & $0.09[-0.08,0.26]$ \\
\hline Kumwenda 2014 & 237 & 60 & $0.1(0.086)$ & 1 & $5.54 \%$ & $0.06[-0.11,0.23]$ \\
\hline Mangani 2015 & 210 & 104 & $0.1(0.095)$ & + & $4.78 \%$ & $0.09[-0.1,0.28]$ \\
\hline Mangani 2015 & 212 & 105 & $0(0.097)$ & 1 & $4.65 \%$ & $0.04[-0.15,0.23]$ \\
\hline Subtotal $(95 \% \mathrm{Cl})$ & & & & 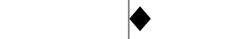 & $54.79 \%$ & $0.07[0,0.14]$ \\
\hline \multicolumn{7}{|c|}{ Heterogeneity: $\mathrm{Tau}^{2}=0 ; \mathrm{Chi}^{2}=14.52, \mathrm{df}=8(\mathrm{P}=0.07) ; \mathrm{I}^{2}=44.9 \%$} \\
\hline \multicolumn{7}{|c|}{ Test for overall effect: $Z=2.07(P=0.04)$} \\
\hline \multicolumn{7}{|c|}{ 3.14.3 More than 12 months } \\
\hline Dewey 2017 & 785 & 816 & $0.1(0.044)$ & + & $11.79 \%$ & $0.08[-0.01,0.17]$ \\
\hline Luby 2018 & 567 & 1104 & $0.2(0.051)$ & $\rightarrow$ & $10.46 \%$ & $0.17[0.07,0.27]$ \\
\hline Null 2018 & 695 & 1536 & $0(0.042)$ & + & $12.15 \%$ & $0.03[-0.05,0.11]$ \\
\hline Subtotal $(95 \% \mathrm{Cl})$ & & & & $\gamma$ & $34.4 \%$ & $0.09[0.01,0.17]$ \\
\hline \multicolumn{7}{|c|}{ Heterogeneity: $\mathrm{Tau}^{2}=0 ; \mathrm{Chi}^{2}=4.54, \mathrm{df}=2(\mathrm{P}=0.1) ; \mathrm{I}^{2}=55.91 \%$} \\
\hline \multicolumn{7}{|c|}{ Test for overall effect: $Z=2.26(P=0.02)$} \\
\hline Total $(95 \% \mathrm{Cl})$ & & & & 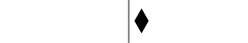 & $100 \%$ & $0.08[0.04,0.13]$ \\
\hline \multicolumn{7}{|c|}{ Heterogeneity: $\mathrm{Tau}^{2}=0 ; \mathrm{Chi}^{2}=21.83, \mathrm{df}=13(\mathrm{P}=0.06) ; \mathrm{I}^{2}=40.44 \%$} \\
\hline \multicolumn{7}{|c|}{ Test for overall effect: $\mathrm{Z}=3.51(\mathrm{P}=0)$} \\
\hline Test for subgroup dif & $d f=1(P=0$. & $\mathrm{I}^{2}=0 \%$ & & & & \\
\hline
\end{tabular}

\section{Comparison 4. LNS versus no intervention: Subgroup analysis by age at follow-up}

\begin{tabular}{lllll}
\hline $\begin{array}{l}\text { Outcome or sub- } \\
\text { group title }\end{array}$ & No. of studies & $\begin{array}{l}\text { No. of partici- } \\
\text { pants }\end{array}$ & Statistical method & Effect size \\
\hline 1 Moderate stunting & 9 & 13492 & Risk Ratio (Random, 95\% Cl) & $0.93[0.88,0.98]$ \\
\hline 1.1 At 12 months & 1 & 564 & Risk Ratio (Random, 95\% Cl) & $1.01[0.86,1.19]$ \\
\hline 1.2 At 18 months & 6 & 7427 & Risk Ratio (Random, 95\% Cl) & $0.95[0.88,1.02]$ \\
\hline 1.3 At 24 months & 3 & Risk Ratio (Random, 95\% Cl) & $0.89[0.81,0.97]$ \\
\hline 2 Severe stunting & 5 & 5501 & Risk Ratio (Random, 95\% Cl) & $0.85[0.74,0.98]$ \\
\hline 2.1 At 12 months & 1 & 6151 & Risk Ratio (Random, 95\% Cl) & $0.96[0.67,1.38]$ \\
\hline 2.2 At 18 months & 2 & 564 & Risk Ratio (Random, 95\% Cl) & $0.86[0.70,1.07]$ \\
\hline 2.3 At 24 months & 2 & 1687 & Risk Ratio (Random, 95\% Cl) & $0.80[0.64,0.99]$ \\
\hline 3 Moderate wasting & 8 & 3900 & Risk Ratio (Random, 95\% Cl) & $0.80[0.73,0.88]$ \\
\hline 3.1 At 18 months & 6 & 13172 & Risk Ratio (Random, 95\% Cl) & $0.79[0.71,0.89]$ \\
\hline 3.2 At 24 months & 3 & 5503 & Risk Ratio (Random, 95\% Cl) & $0.83[0.69,0.99]$ \\
\hline
\end{tabular}




\begin{tabular}{|c|c|c|c|c|}
\hline $\begin{array}{l}\text { Outcome or sub- } \\
\text { group title }\end{array}$ & No. of studies & $\begin{array}{l}\text { No. of partici- } \\
\text { pants }\end{array}$ & Statistical method & Effect size \\
\hline 4 Severe wasting & 3 & 2329 & Risk Ratio (Random, 95\% Cl) & $1.27[0.66,2.46]$ \\
\hline 4.1 At 12 months & 1 & 563 & Risk Ratio (Random, 95\% Cl) & $0.78[0.12,4.93]$ \\
\hline 4.2 At 18 months & 2 & 1766 & Risk Ratio (Random, 95\% Cl) & $1.39[0.67,2.87]$ \\
\hline $\begin{array}{l}5 \text { Moderate under- } \\
\text { weight }\end{array}$ & 8 & 13073 & Risk Ratio (Random, 95\% Cl) & $0.85[0.81,0.90]$ \\
\hline 5.1 At 18 months & 6 & 7532 & Risk Ratio (Random, 95\% Cl) & $0.85[0.78,0.92]$ \\
\hline 5.2 At 24 months & 3 & 5541 & Risk Ratio (Random, 95\% Cl) & $0.87[0.79,0.95]$ \\
\hline $\begin{array}{l}6 \text { Severe underweight: } \\
\text { at } 18 \text { months }\end{array}$ & 2 & 1729 & Risk Ratio (Random, 95\% Cl) & $0.78[0.54,1.13]$ \\
\hline 7 Anaemia & 5 & 2332 & Risk Ratio (M-H, Random, 95\% Cl) & $0.79[0.69,0.90]$ \\
\hline 7.1 At 12 months & 2 & 1176 & Risk Ratio (M-H, Random, 95\% Cl) & $0.55[0.20,1.47]$ \\
\hline 7.2 At 18 months & 3 & 1156 & Risk Ratio (M-H, Random, 95\% Cl) & $0.80[0.71,0.91]$ \\
\hline 7.3 At 36 months & 0 & 0 & Risk Ratio (M-H, Random, 95\% Cl) & $0.0[0.0,0.0]$ \\
\hline $\begin{array}{l}8 \text { Adverse effects: At } \\
18 \text { months }\end{array}$ & 3 & 3382 & Risk Ratio (Random, 95\% Cl) & $0.86[0.74,1.01]$ \\
\hline $\begin{array}{l}9 \text { Mid-upper arm cir- } \\
\text { cumference (MUAC) }\end{array}$ & 6 & 8187 & Std. Mean Difference (Random, 95\% Cl) & $0.13[0.05,0.22]$ \\
\hline 9.1 At 12 months & 1 & 1193 & Std. Mean Difference (Random, 95\% Cl) & $0.15[0.06,0.24]$ \\
\hline 9.2 At 18 months & 3 & 4355 & Std. Mean Difference (Random, 95\% Cl) & $0.12[-0.11,0.34]$ \\
\hline 9.3 At 24 months & 1 & 1601 & Std. Mean Difference (Random, 95\% Cl) & $0.04[-0.04,0.12]$ \\
\hline 9.4 At 36 months & 1 & 1038 & Std. Mean Difference (Random, 95\% Cl) & $0.2[0.06,0.34]$ \\
\hline $\begin{array}{l}10 \text { Serum haemoglo- } \\
\text { bin }(\mathrm{g} / \mathrm{L})\end{array}$ & 4 & 4518 & Mean Difference (IV, Random, 95\% CI) & $5.78[2.27,9.30]$ \\
\hline 10.1 At 12 months & 1 & 194 & Mean Difference (IV, Random, 95\% CI) & $8.5[4.46,12.54]$ \\
\hline 10.2 At 18 months & 2 & 3342 & Mean Difference (IV, Random, 95\% CI) & $5.96[0.08,11.84]$ \\
\hline 10.3 At 36 months & 1 & 982 & Mean Difference (IV, Random, 95\% Cl) & $3.30[1.42,5.18]$ \\
\hline 11 Mortality & 3 & 3321 & Risk Ratio (Random, 95\% Cl) & $0.93[0.63,1.37]$ \\
\hline 11.1 At 12 months & 1 & 1932 & Risk Ratio (Random, 95\% Cl) & $0.85[0.56,1.30]$ \\
\hline 11.2 At 18 months & 2 & 1389 & Risk Ratio (Random, 95\% Cl) & $1.10[0.42,2.89]$ \\
\hline $12 \mathrm{HAZ}$ & 12 & 15795 & Std. Mean Difference (Random, 95\% Cl) & $0.10[0.05,0.15]$ \\
\hline
\end{tabular}

Preventive lipid-based nutrient supplements given with complementary foods to infants and young children 6 to 23 months of age for 


\begin{tabular}{|c|c|c|c|c|}
\hline $\begin{array}{l}\text { Outcome or sub- } \\
\text { group title }\end{array}$ & No. of studies & $\begin{array}{l}\text { No. of partici- } \\
\text { pants }\end{array}$ & Statistical method & Effect size \\
\hline $\begin{array}{l}12.1 \text { At } 12 \text { to } 15 \\
\text { months }\end{array}$ & 3 & 1103 & Std. Mean Difference (Random, 95\% Cl) & $0.07[-0.02,0.15]$ \\
\hline 12.2 At 18 months & 6 & 8153 & Std. Mean Difference (Random, 95\% Cl) & $0.08[0.00,0.16]$ \\
\hline 12.3 At 24 months & 3 & 5501 & Std. Mean Difference (Random, 95\% Cl) & $0.14[0.03,0.25]$ \\
\hline 12.4 At 36 months & 1 & 1038 & Std. Mean Difference (Random, 95\% Cl) & $0.27[0.10,0.44]$ \\
\hline 13 WAZ & 10 & 12188 & Std. Mean Difference (Random, 95\% Cl) & $0.10[0.04,0.16]$ \\
\hline $\begin{array}{l}13.1 \text { At } 12 \text { to } 15 \\
\text { months }\end{array}$ & 3 & 1103 & Std. Mean Difference (Random, 95\% Cl) & $0.09[-0.00,0.19]$ \\
\hline 13.2 At 18 months & 5 & 5544 & Std. Mean Difference (Random, 95\% Cl) & $0.05[-0.06,0.17]$ \\
\hline 13.3 At 24 months & 3 & 5541 & Std. Mean Difference (Random, 95\% Cl) & $0.13[0.03,0.23]$ \\
\hline $14 \mathrm{WHZ}$ & 10 & 12894 & Std. Mean Difference (Random, 95\% Cl) & $0.12[0.02,0.22]$ \\
\hline $\begin{array}{l}14.1 \text { At } 12 \text { to } 15 \\
\text { months }\end{array}$ & 2 & 809 & Std. Mean Difference (Random, 95\% Cl) & $0.10[-0.02,0.22]$ \\
\hline 14.2 At 18 months & 5 & 5544 & Std. Mean Difference (Random, 95\% Cl) & $0.13[-0.05,0.31]$ \\
\hline 14.3 At 24 months & 3 & 5503 & Std. Mean Difference (Random, 95\% Cl) & $0.09[0.01,0.17]$ \\
\hline 14.4 At 36 months & 1 & 1038 & Std. Mean Difference (Random, 95\% Cl) & $0.04[-0.08,0.16]$ \\
\hline
\end{tabular}

\section{Analysis 4.1. Comparison 4 LNS versus no intervention: Subgroup analysis by age at follow-up, Outcome 1 Moderate stunting.}

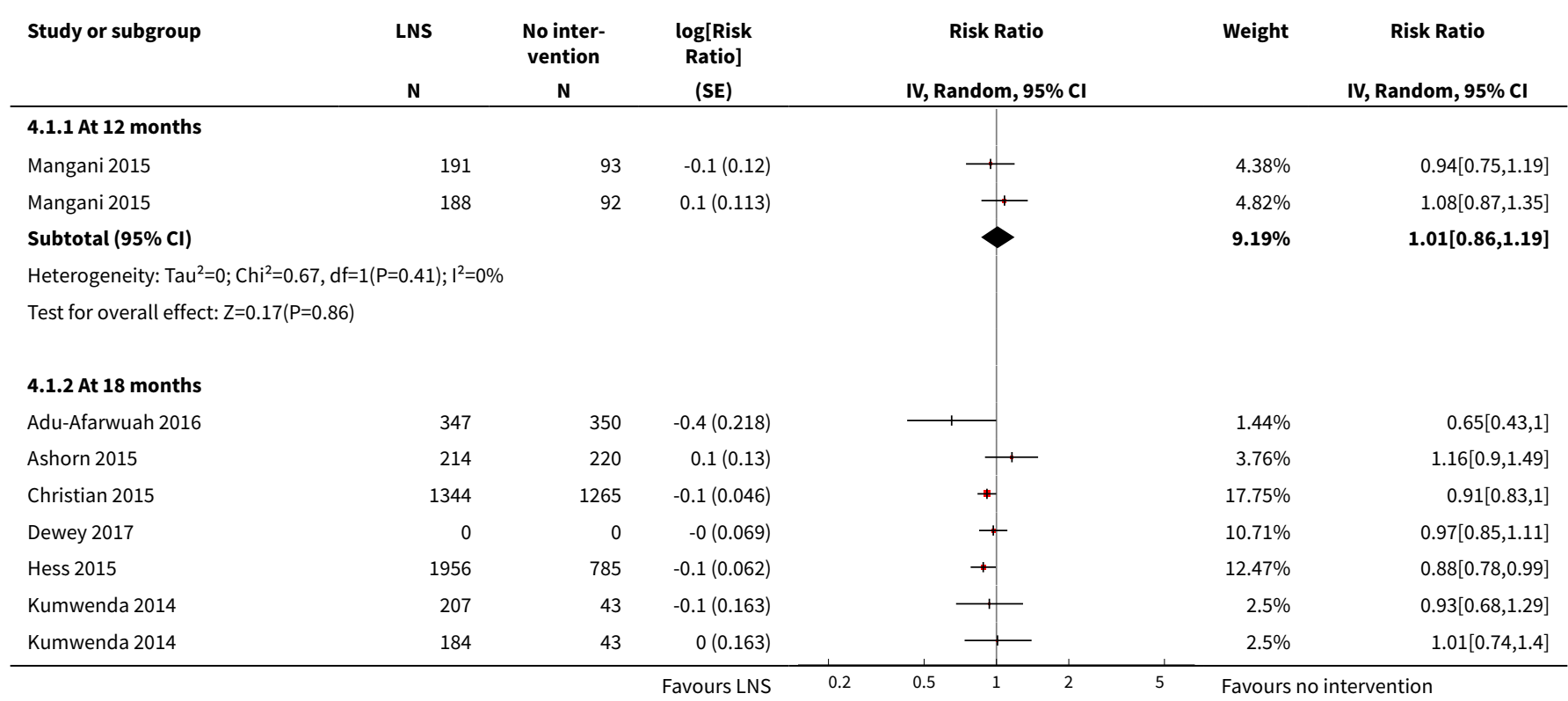




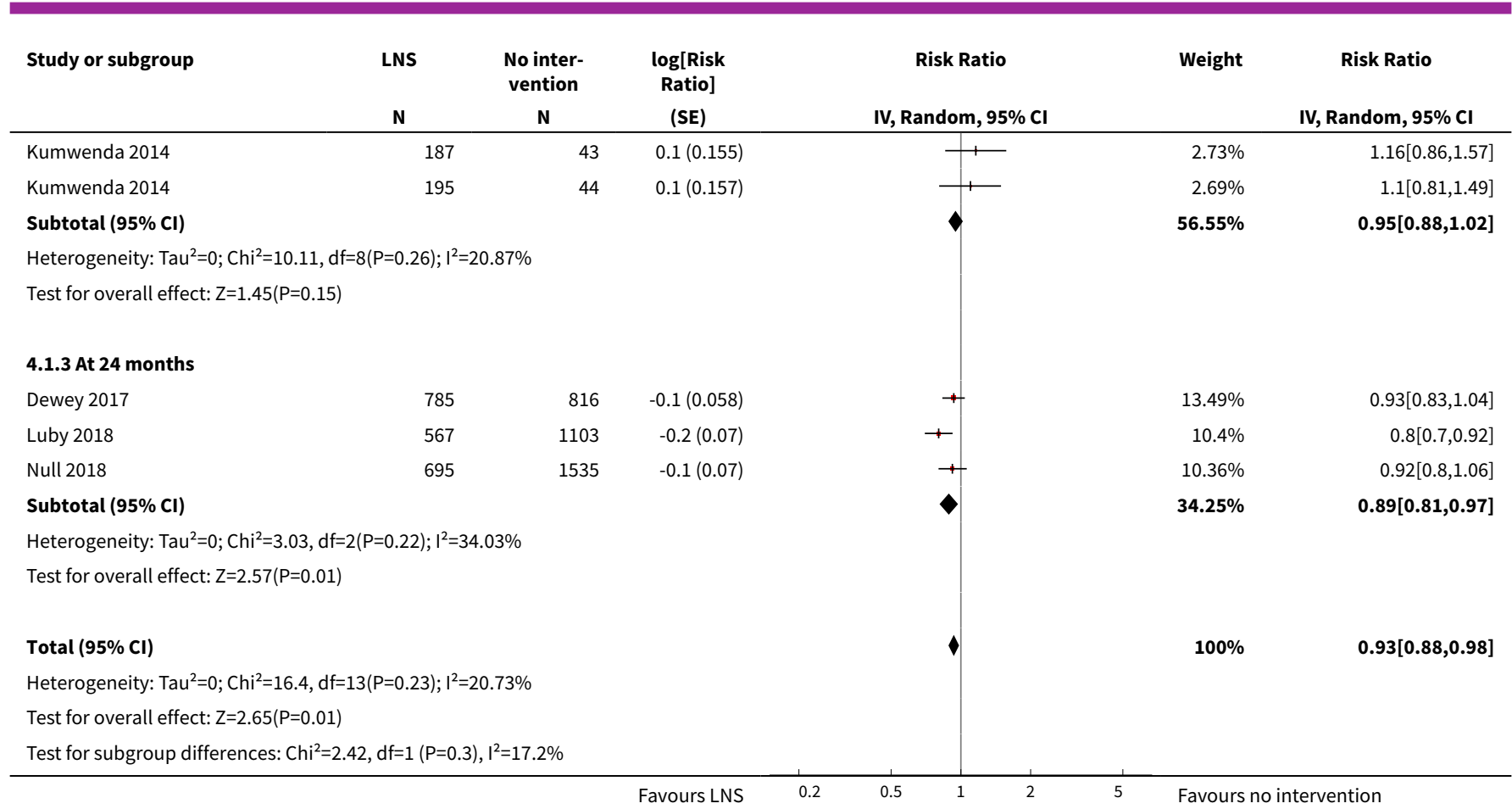

\section{Analysis 4.2. Comparison 4 LNS versus no intervention: Subgroup analysis by age at follow-up, Outcome 2 Severe stunting.}

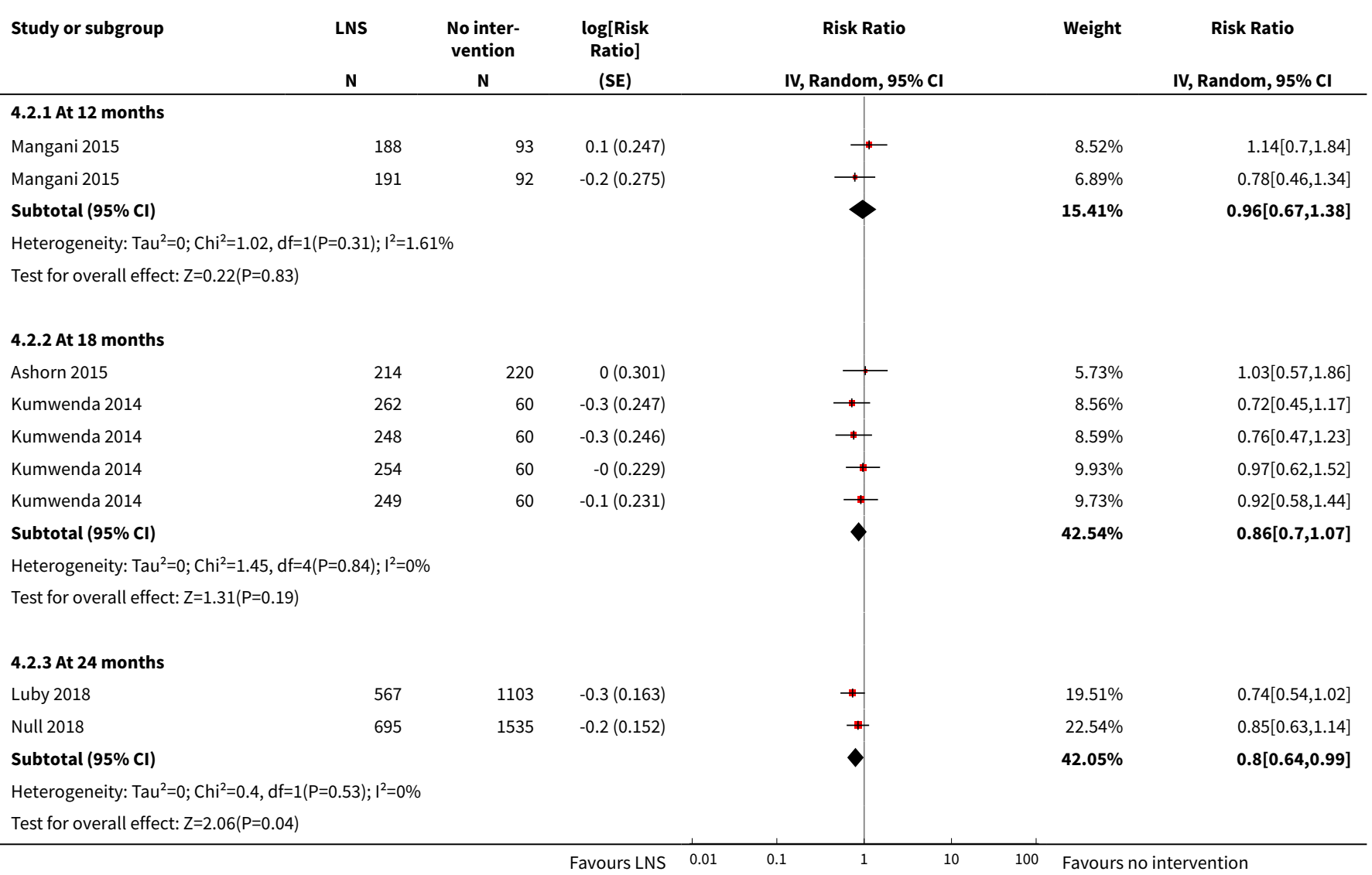

Preventive lipid-based nutrient supplements given with complementary foods to infants and young children 6 to 23 months of age for 


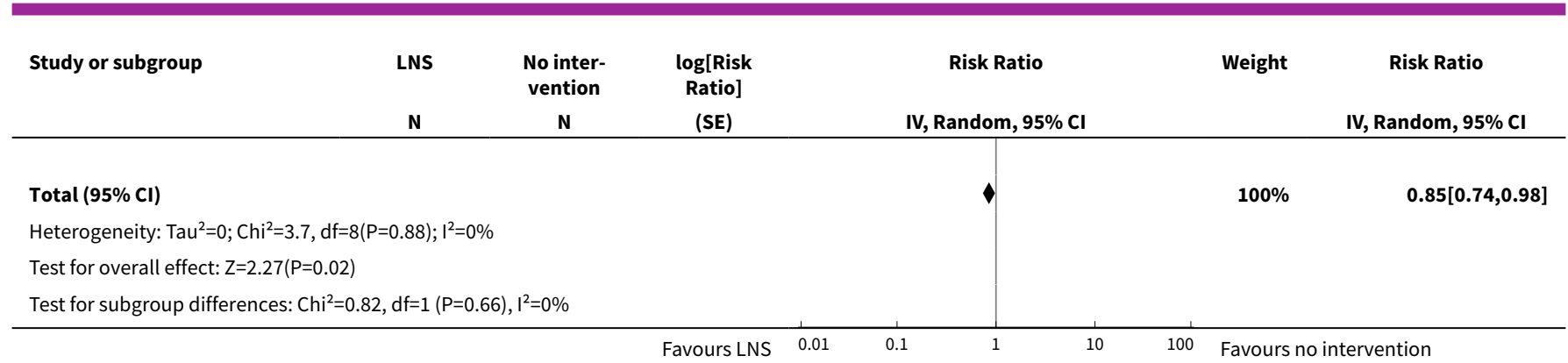

\section{Analysis 4.3. Comparison 4 LNS versus no intervention: Subgroup analysis by age at follow-up, Outcome 3 Moderate wasting.}

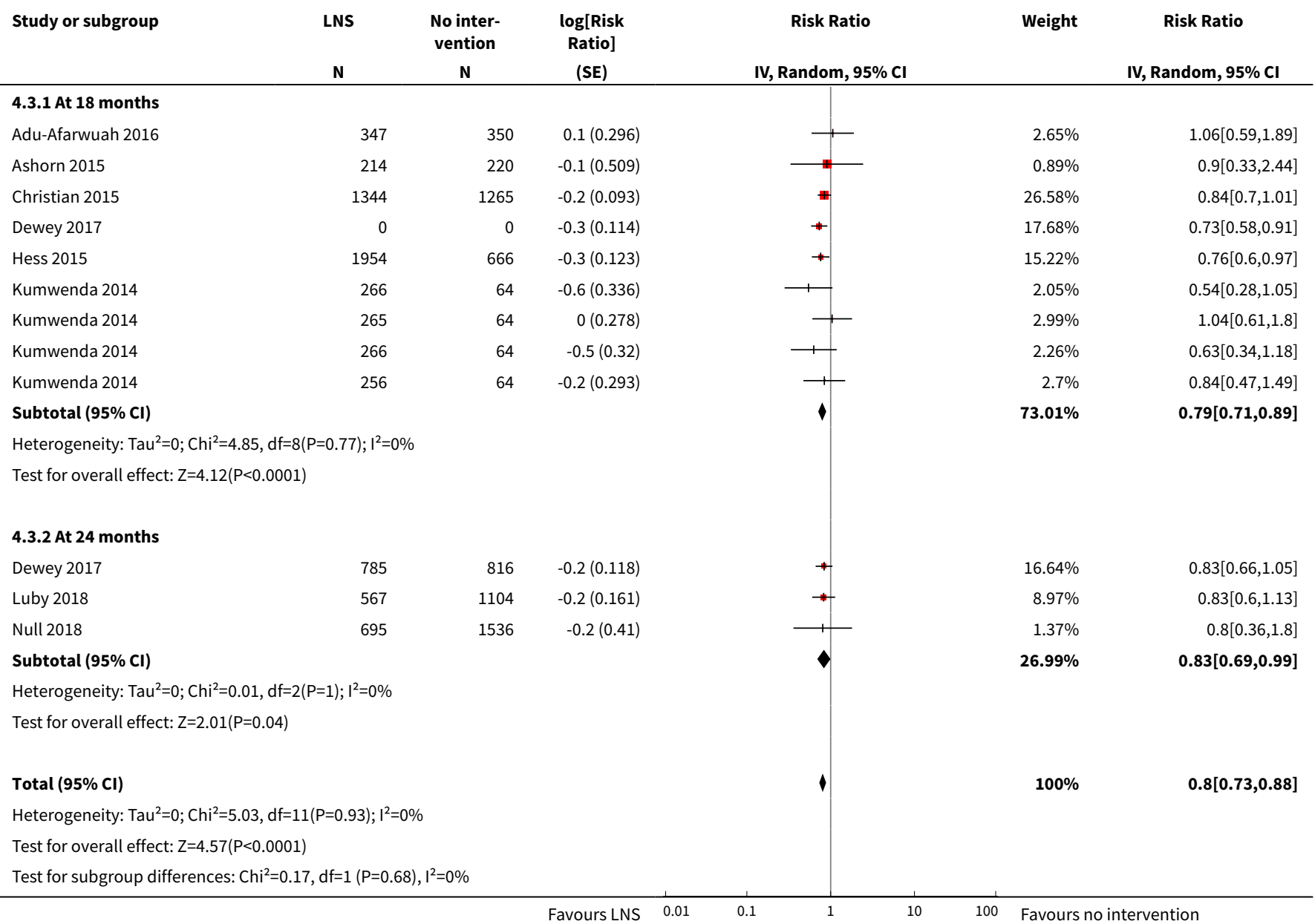

Preventive lipid-based nutrient supplements given with complementary foods to infants and young children 6 to 23 months of age for 


\section{Analysis 4.4. Comparison 4 LNS versus no intervention: Subgroup} analysis by age at follow-up, Outcome 4 Severe wasting.

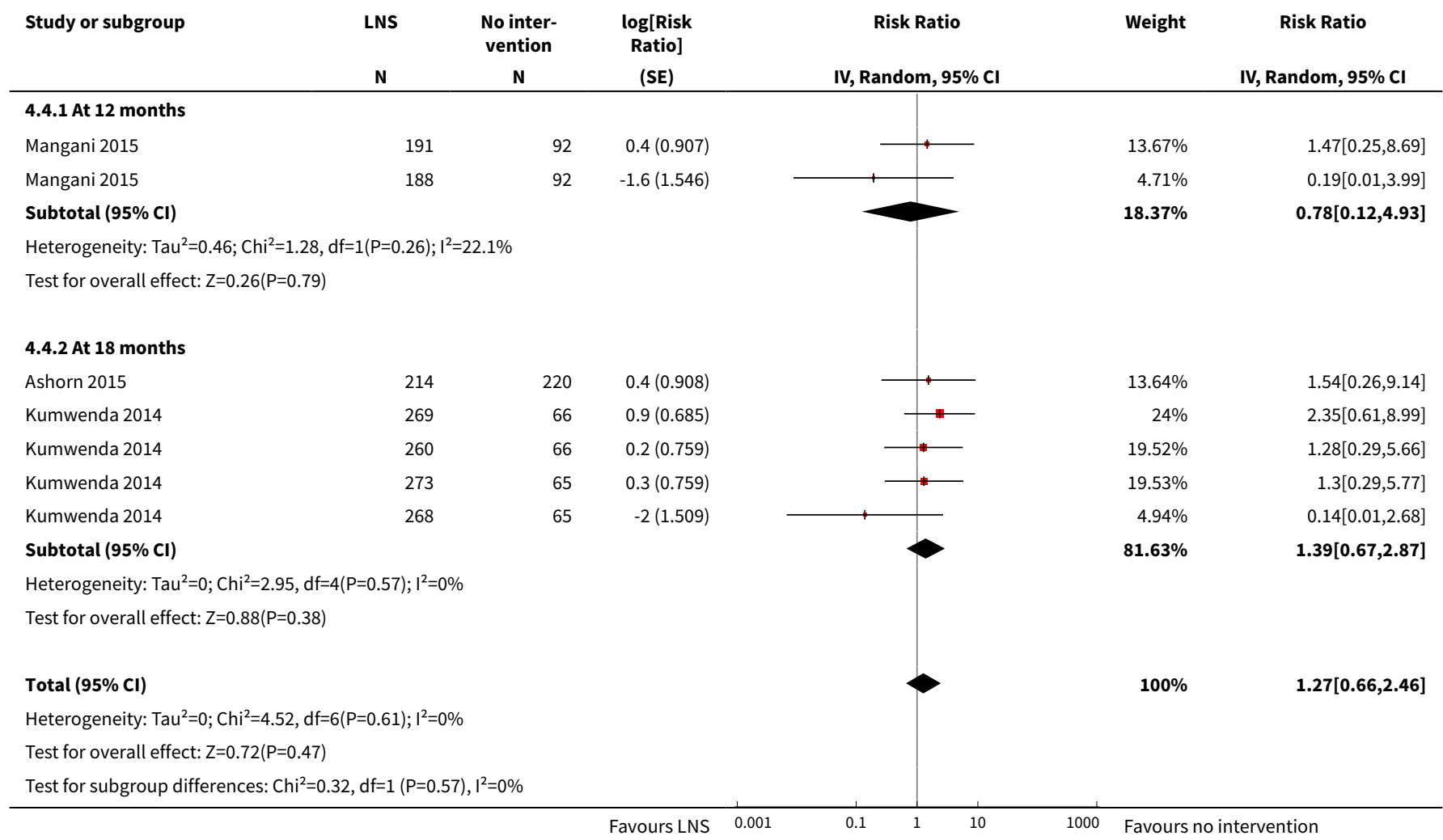

\section{Analysis 4.5. Comparison 4 LNS versus no intervention: Subgroup analysis by age at follow-up, Outcome 5 Moderate underweight.}

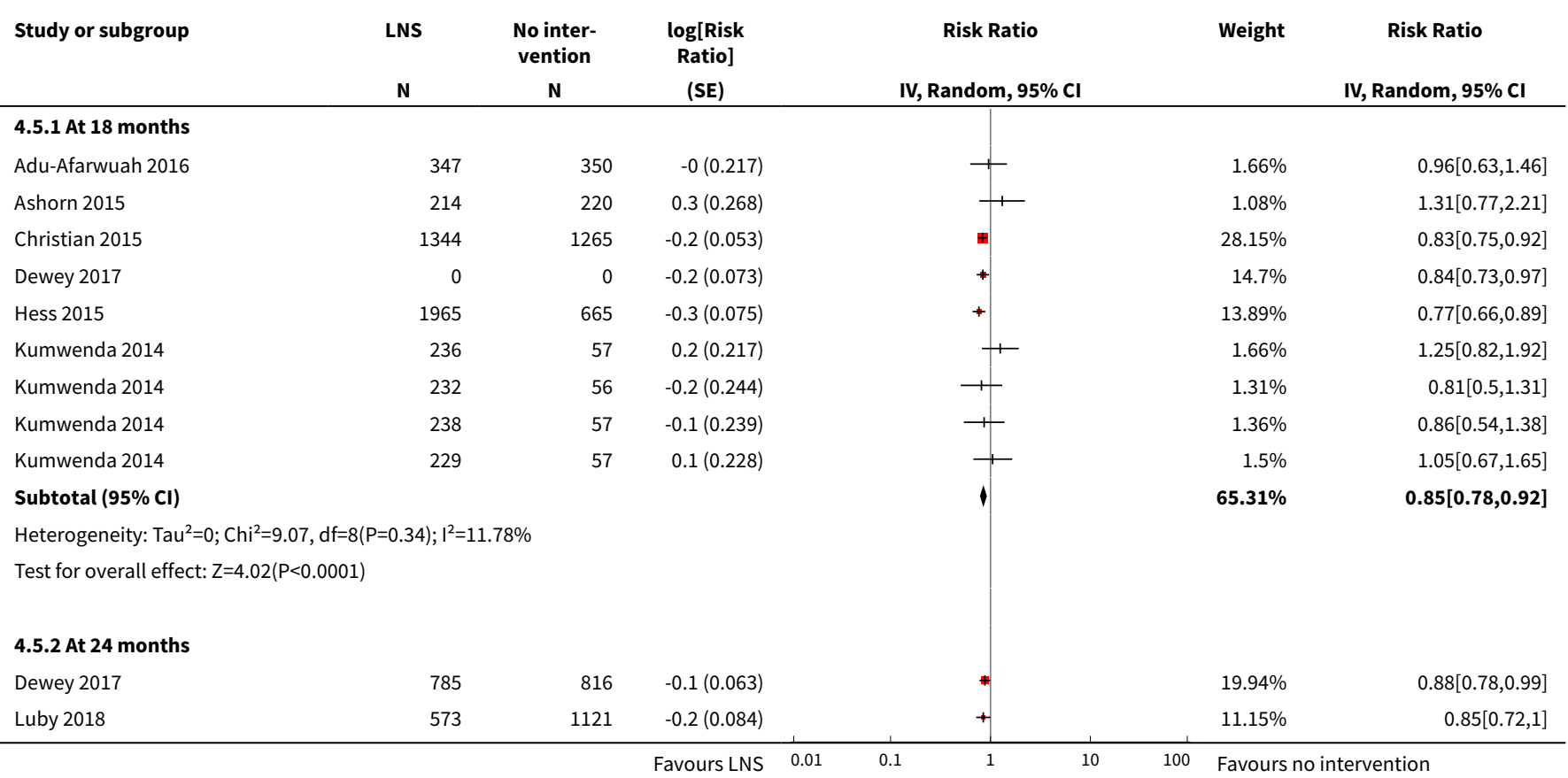

Preventive lipid-based nutrient supplements given with complementary foods to infants and young children 6 to 23 months of age for 


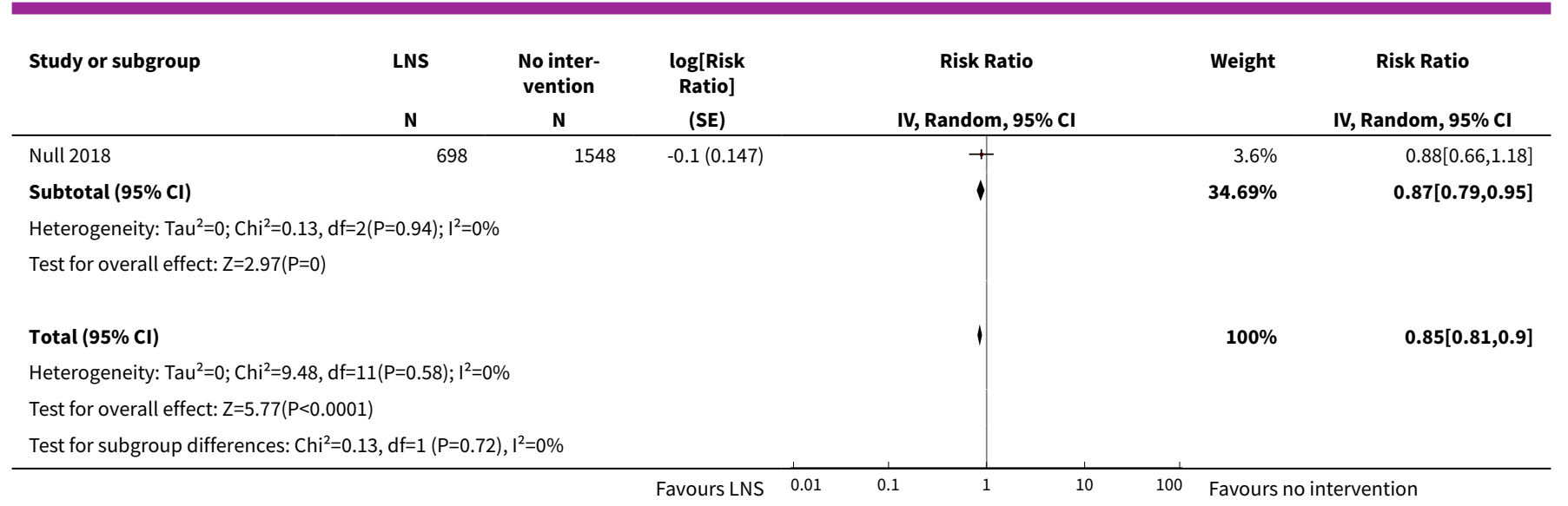

Analysis 4.6. Comparison 4 LNS versus no intervention: Subgroup analysis by age at follow-up, Outcome 6 Severe underweight: at 18 months.

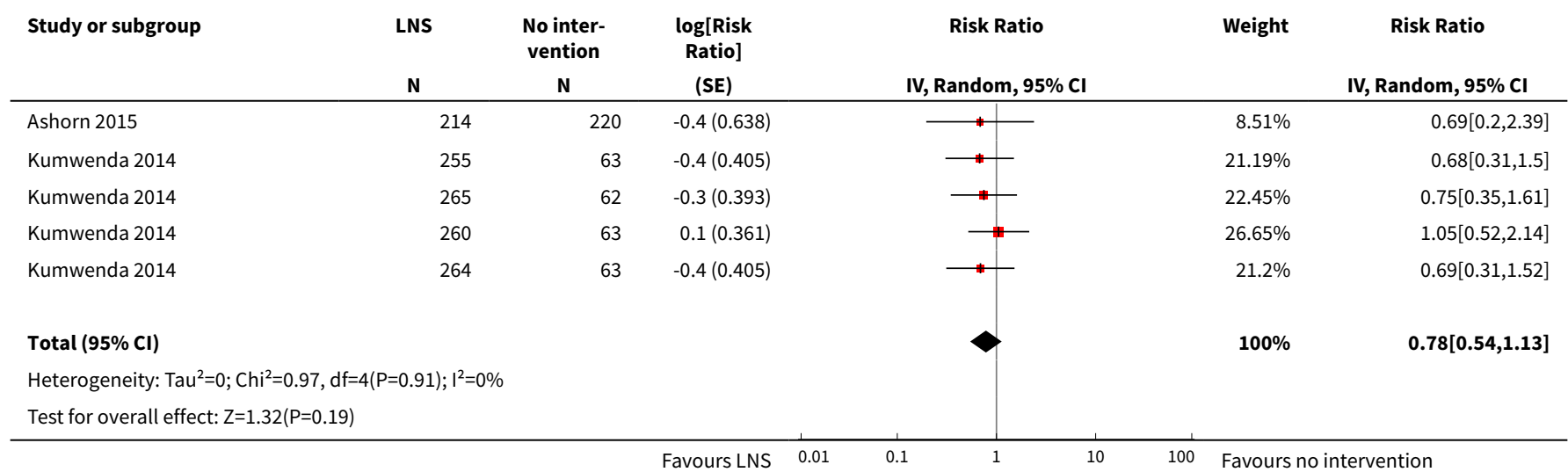

Analysis 4.7. Comparison 4 LNS versus no intervention: Subgroup analysis by age at follow-up, Outcome 7 Anaemia.

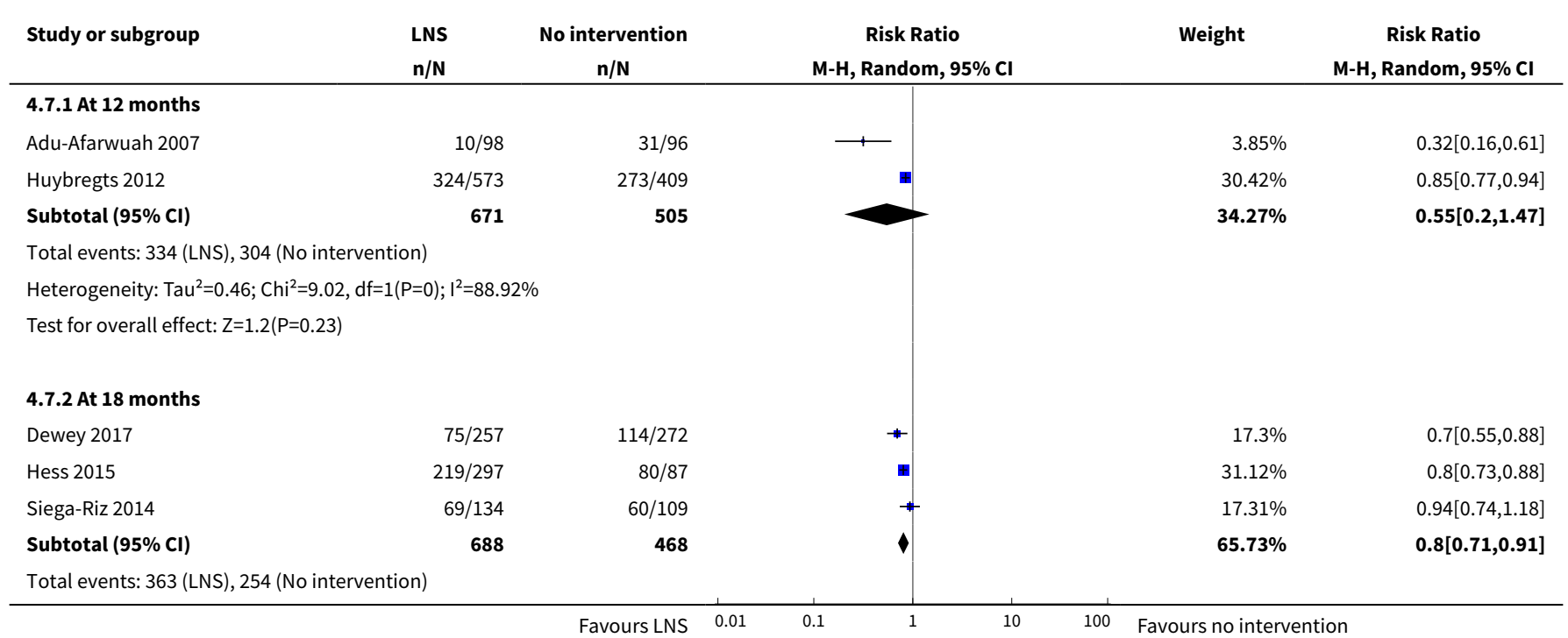




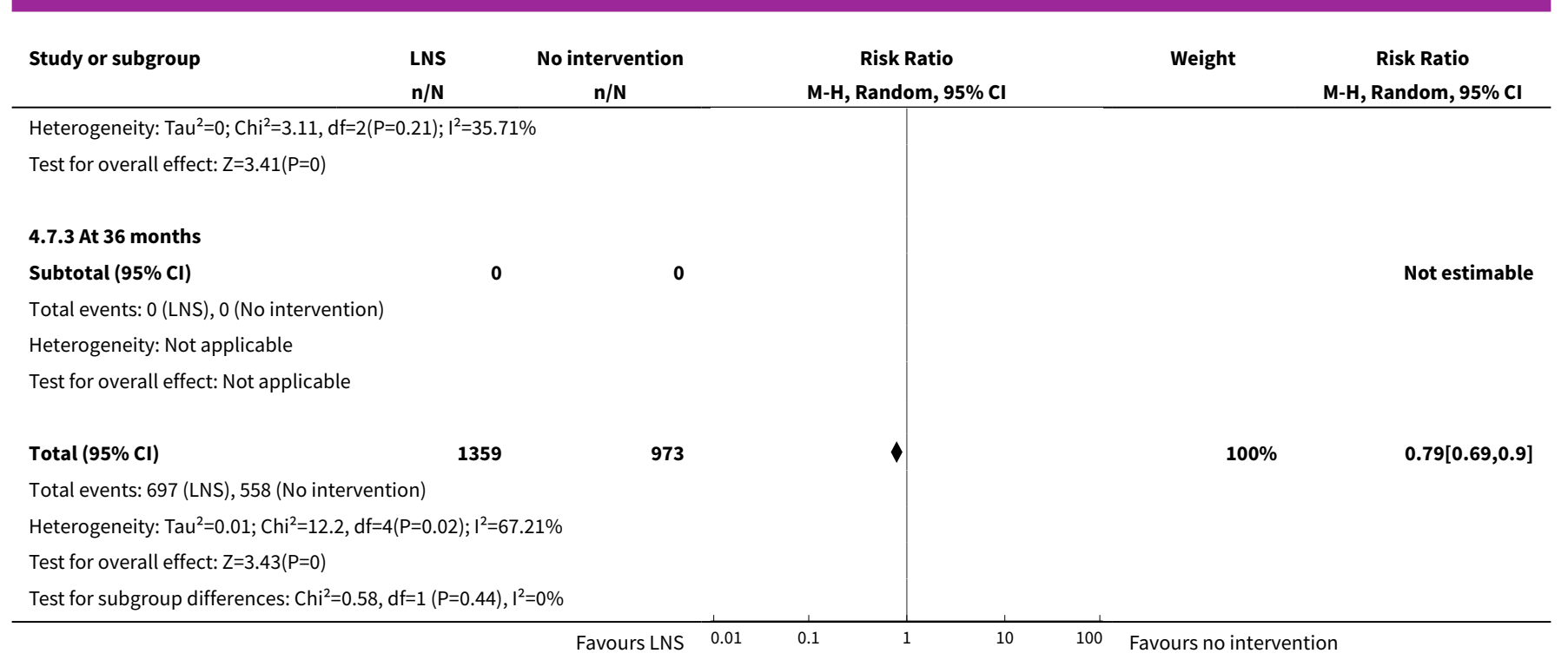

Analysis 4.8. Comparison 4 LNS versus no intervention: Subgroup analysis by age at follow-up, Outcome 8 Adverse effects: At 18 months.

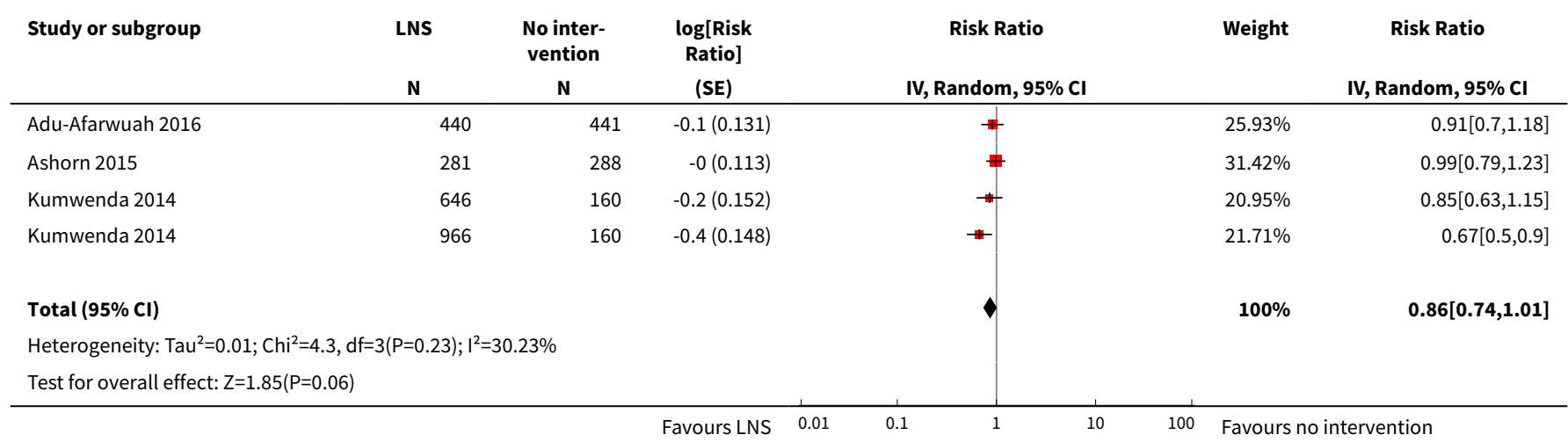

\section{Analysis 4.9. Comparison 4 LNS versus no intervention: Subgroup analysis by age at follow-up, Outcome 9 Mid-upper arm circumference (MUAC).}

\begin{tabular}{|c|c|c|c|c|c|c|}
\hline Study or subgroup & $\begin{array}{l}\text { LNS } \\
\mathrm{N} \\
\end{array}$ & $\begin{array}{l}\text { No Inter- } \\
\text { vention }\end{array}$ & $\begin{array}{l}\text { Std. Mean } \\
\text { Difference } \\
\text { (SE) } \\
\end{array}$ & $\begin{array}{l}\text { Std. Mean Difference } \\
\text { IV, Random, } 95 \% \mathrm{Cl}\end{array}$ & Weight & $\begin{array}{l}\text { Std. Mean Difference } \\
\text { IV, Random, } 95 \% \mathrm{Cl}\end{array}$ \\
\hline \multicolumn{7}{|l|}{ 4.9.1 At 12 months } \\
\hline Kumwenda 2014 & 237 & 60 & $0.2(0.087)$ & + & $10.12 \%$ & $0.2[0.03,0.37]$ \\
\hline Kumwenda 2014 & 241 & 60 & $0.2(0.092)$ & 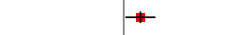 & $9.65 \%$ & $0.2[0.02,0.38]$ \\
\hline Kumwenda 2014 & 233 & 60 & $0.1(0.088)$ & + & $10.03 \%$ & $0.1[-0.07,0.27]$ \\
\hline Subtotal $(95 \% \mathrm{CI})$ & & & & 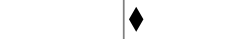 & $39.88 \%$ & $0.15[0.06,0.24]$ \\
\hline \multicolumn{7}{|c|}{ Heterogeneity: $\operatorname{Tau}^{2}=0 ; \mathrm{Chi}^{2}=1.29, \mathrm{df}=3(\mathrm{P}=0.73) ; \mathrm{I}^{2}=0 \%$} \\
\hline \multicolumn{7}{|c|}{ Test for overall effect: $\mathrm{Z}=3.38(\mathrm{P}=0)$} \\
\hline
\end{tabular}

Preventive lipid-based nutrient supplements given with complementary foods to infants and young children 6 to 23 months of age for 


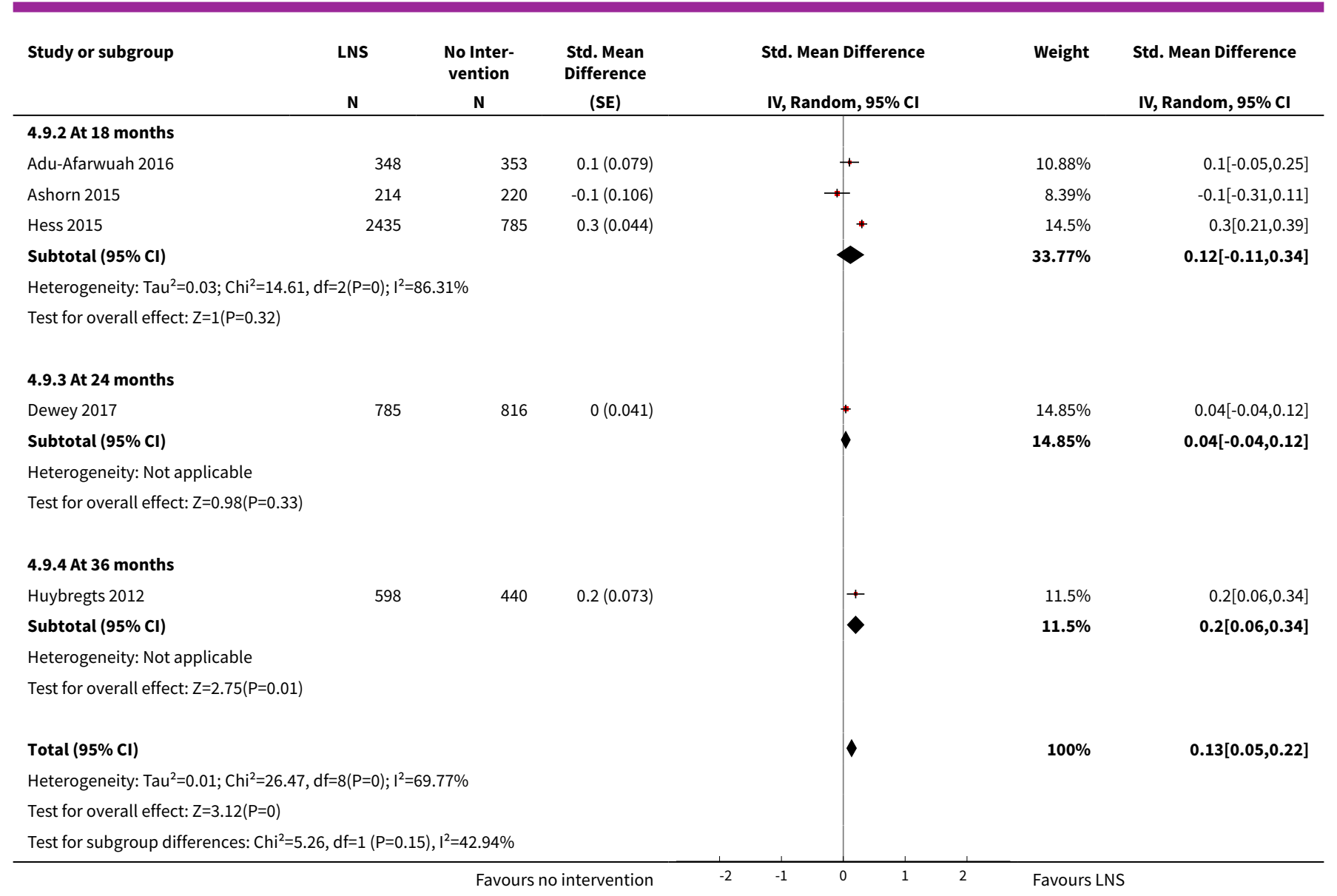

Analysis 4.10. Comparison 4 LNS versus no intervention: Subgroup analysis by age at follow-up, Outcome 10 Serum haemoglobin $(\mathrm{g} / \mathrm{L})$.

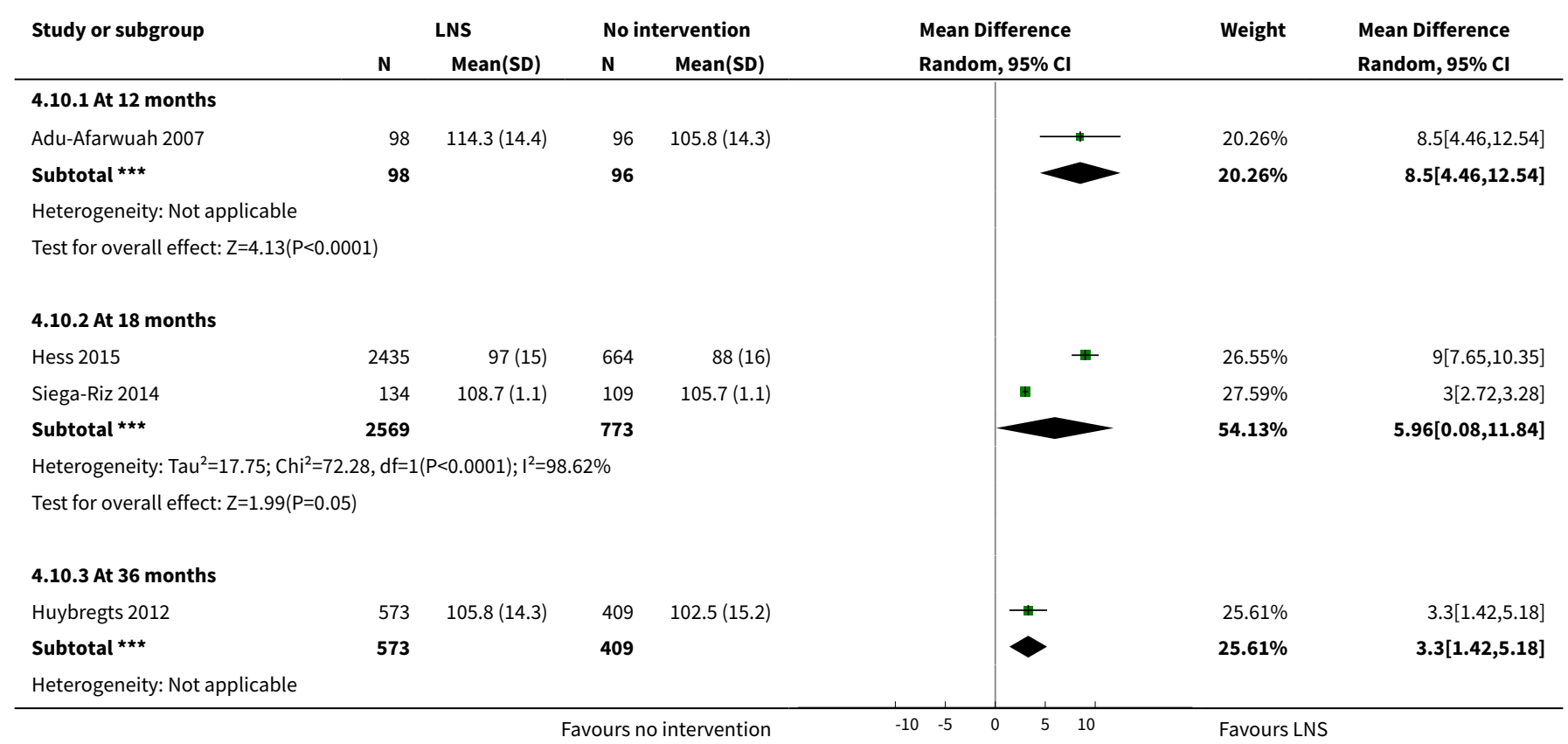

Preventive lipid-based nutrient supplements given with complementary foods to infants and young children 6 to 23 months of age for 


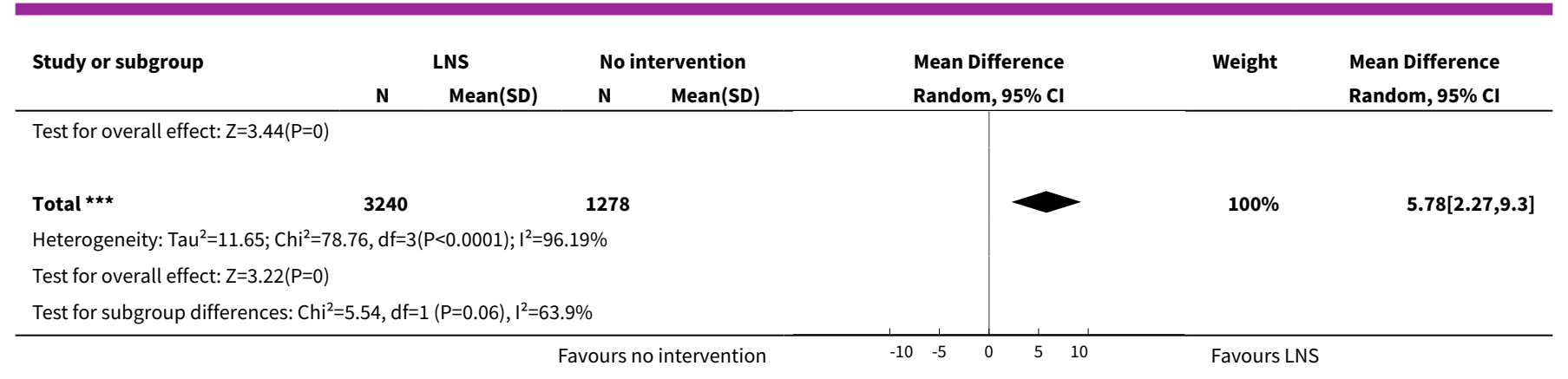

Analysis 4.11. Comparison 4 LNS versus no intervention: Subgroup analysis by age at follow-up, Outcome 11 Mortality.

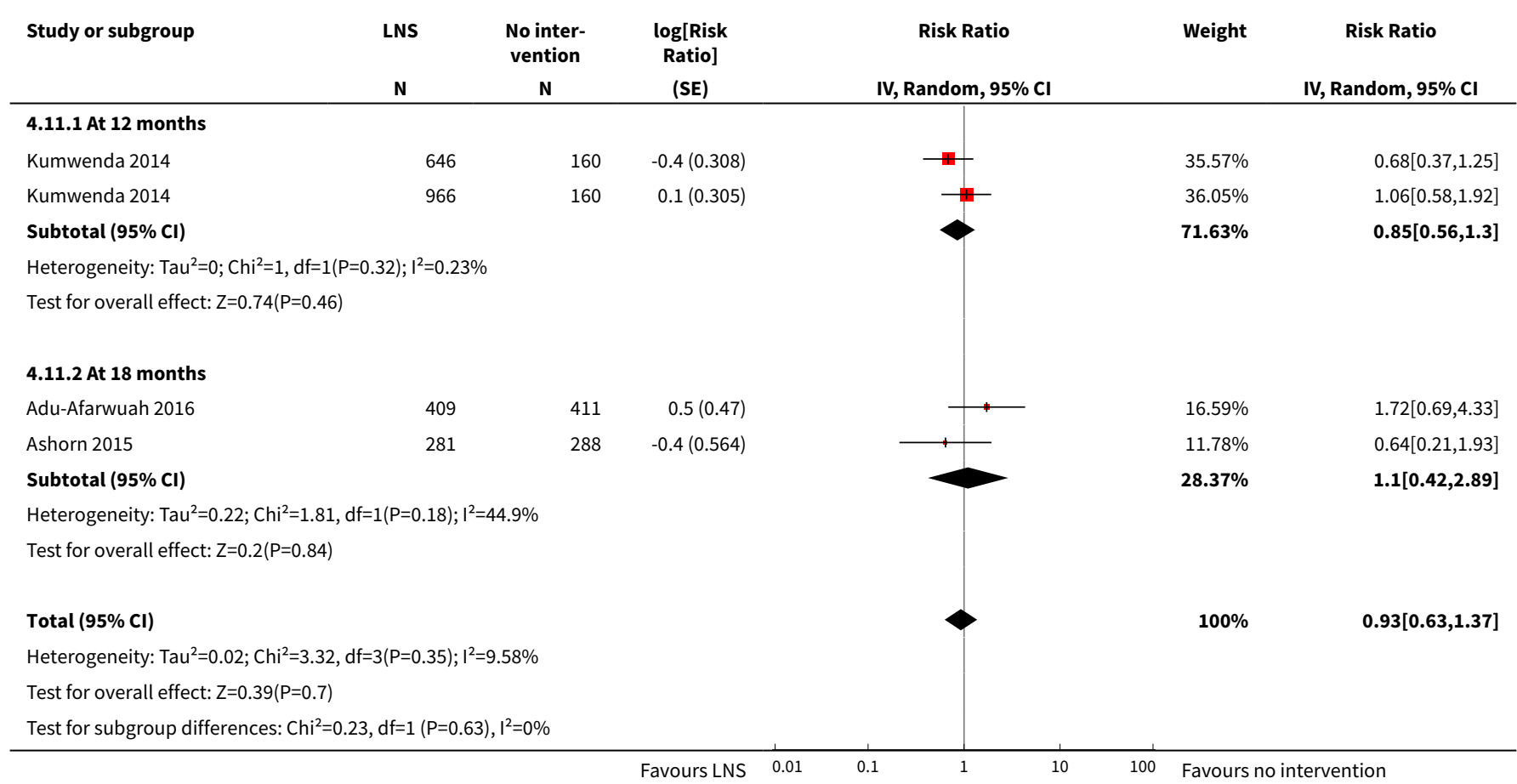

Analysis 4.12. Comparison 4 LNS versus no intervention: Subgroup analysis by age at follow-up, Outcome 12 HAZ.

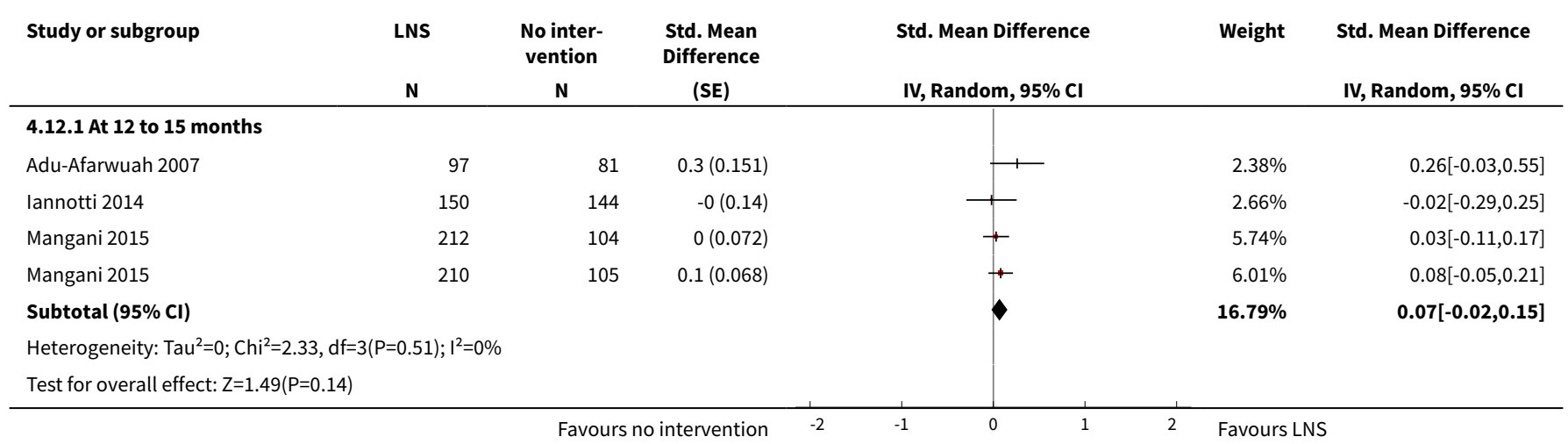




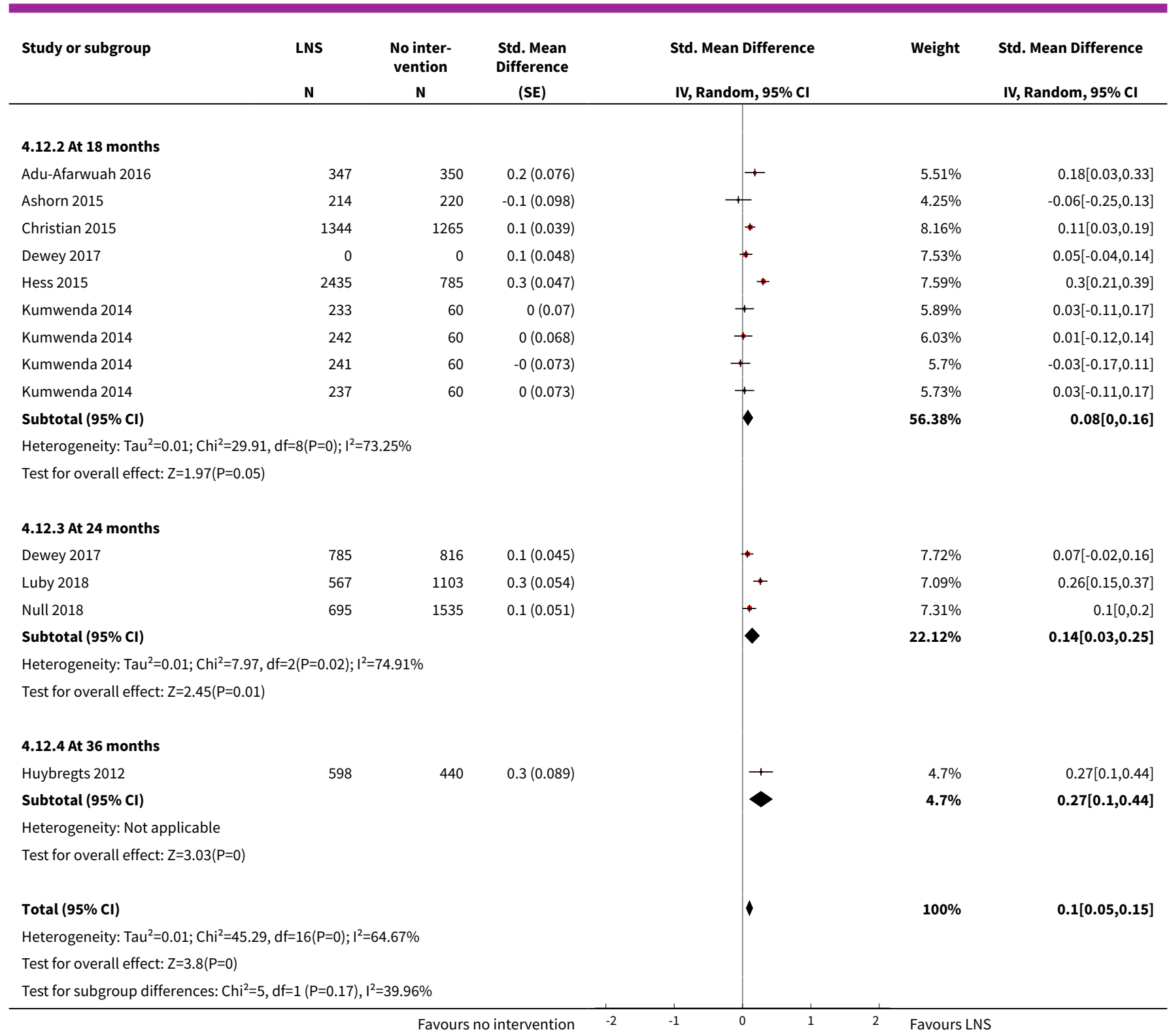

Analysis 4.13. Comparison 4 LNS versus no intervention: Subgroup analysis by age at follow-up, Outcome 13 WAZ.

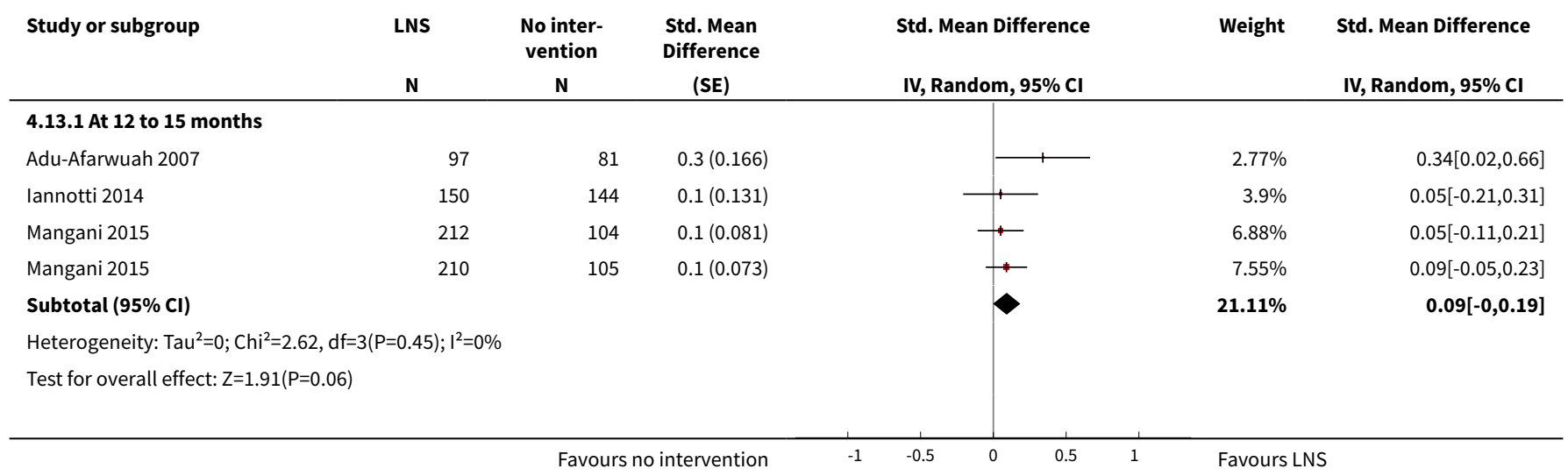




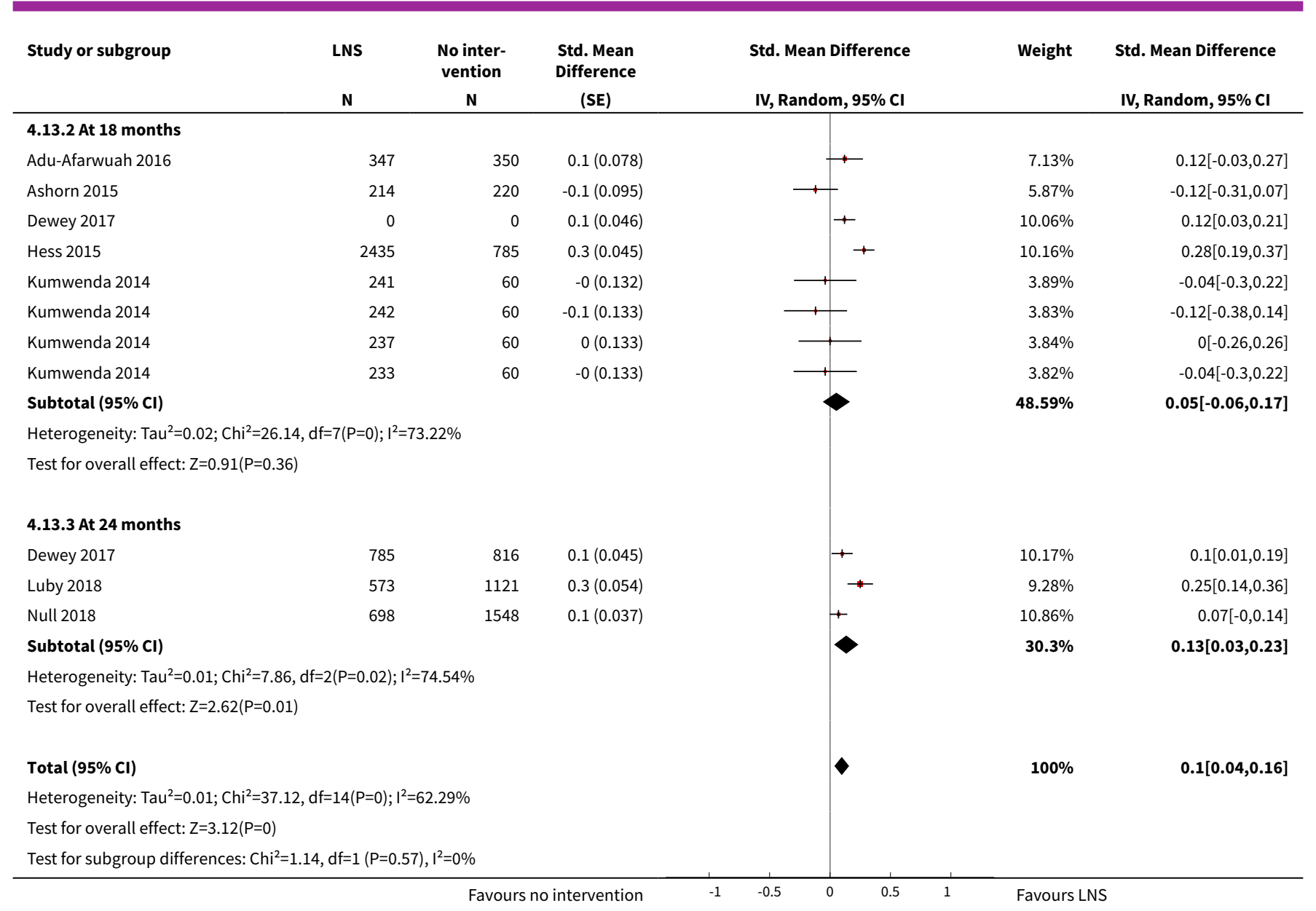

Analysis 4.14. Comparison 4 LNS versus no intervention: Subgroup analysis by age at follow-up, Outcome 14 WHZ.

\begin{tabular}{|c|c|c|c|c|c|c|}
\hline Study or subgroup & $\begin{array}{l}\text { LNS } \\
\mathrm{N}\end{array}$ & $\begin{array}{c}\text { No inter- } \\
\text { vention } \\
\mathbf{N}\end{array}$ & $\begin{array}{l}\text { Std. Mean } \\
\text { Difference } \\
\quad \text { (SE) }\end{array}$ & $\begin{array}{l}\text { Std. Mean Difference } \\
\text { IV, Random, } 95 \% \mathrm{Cl}\end{array}$ & Weight & $\begin{array}{l}\text { Std. Mean Difference } \\
\text { IV, Random, } 95 \% \mathrm{CI}\end{array}$ \\
\hline \multicolumn{7}{|c|}{ 4.14.1 At 12 to 15 months } \\
\hline Adu-Afarwuah 2007 & 97 & 81 & $0.3(0.166)$ & 1 & $4.39 \%$ & $0.31[-0.01,0.63]$ \\
\hline Mangani 2015 & 212 & 104 & $0(0.097)$ & $\rightarrow$ & $6.19 \%$ & $0.04[-0.15,0.23]$ \\
\hline Mangani 2015 & 210 & 105 & $0.1(0.095)$ & $\longrightarrow$ & $6.24 \%$ & $0.09[-0.1,0.28]$ \\
\hline \multicolumn{7}{|c|}{ Heterogeneity: $\mathrm{Tau}^{2}=0 ; \mathrm{Chi}^{2}=2, \mathrm{df}=2(\mathrm{P}=0.37) ; \mathrm{I}^{2}=0.12 \%$} \\
\hline \multicolumn{7}{|c|}{ Test for overall effect: $Z=1.6(P=0.11)$} \\
\hline \multicolumn{7}{|l|}{ 4.14.2 At 18 months } \\
\hline Adu-Afarwuah 2016 & 347 & 350 & $0.1(0.077)$ & + & $6.74 \%$ & $0.05[-0.1,0.2]$ \\
\hline Ashorn 2015 & 214 & 220 & $-0.1(0.096)$ & $\rightarrow$ & $6.23 \%$ & $-0.13[-0.32,0.06]$ \\
\hline Hess 2015 & 2435 & 785 & $0.2(0.041)$ & + & $7.52 \%$ & $0.2[0.12,0.28]$ \\
\hline Kumwenda 2014 & 242 & 60 & $-0(0.092)$ & $\leftarrow$ & $6.33 \%$ & $-0.01[-0.19,0.17]$ \\
\hline Kumwenda 2014 & 237 & 60 & $0.1(0.086)$ & + & $6.48 \%$ & $0.06[-0.11,0.23]$ \\
\hline Kumwenda 2014 & 233 & 60 & $0.1(0.086)$ & + & $6.48 \%$ & $0.09[-0.08,0.26]$ \\
\hline Kumwenda 2014 & 241 & 60 & $0.1(0.087)$ & + & $6.47 \%$ & $0.14[-0.03,0.31]$ \\
\hline
\end{tabular}




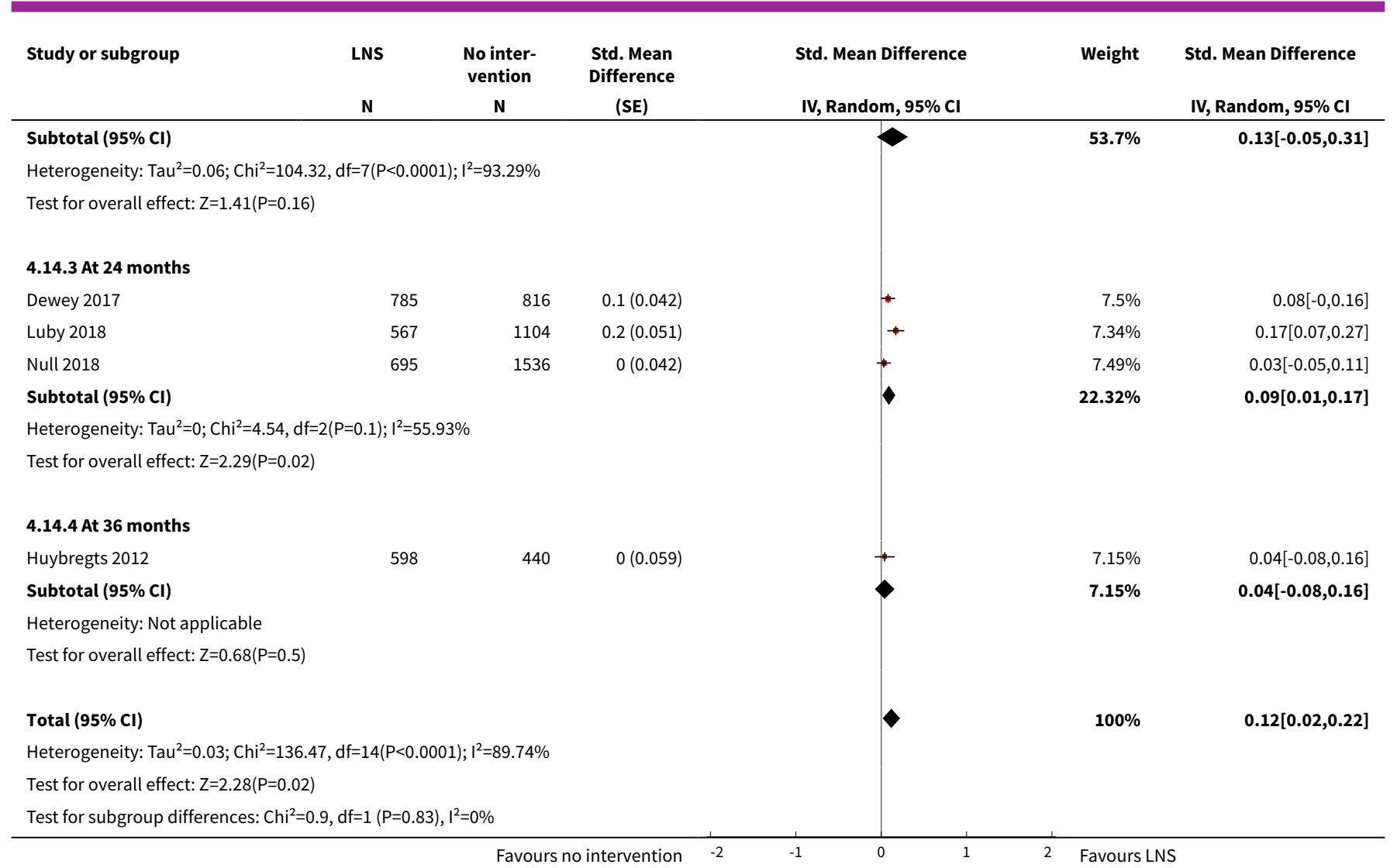

\section{Comparison 5. LNS versus fortified blended food (FBF)}

\begin{tabular}{|c|c|c|c|c|}
\hline $\begin{array}{l}\text { Outcome or sub- } \\
\text { group title }\end{array}$ & No. of studies & $\begin{array}{l}\text { No. of partici- } \\
\text { pants }\end{array}$ & Statistical method & Effect size \\
\hline 1 Moderate stunting & 3 & 2828 & Risk Ratio (Random, 95\% Cl) & $0.89[0.82,0.97]$ \\
\hline 2 Severe stunting & 2 & 729 & Risk Ratio (Random, 95\% Cl) & $0.41[0.12,1.42]$ \\
\hline 3 Moderate wasting & 2 & 2290 & Risk Ratio (Random, 95\% Cl) & $0.79[0.65,0.97]$ \\
\hline 4 Severe wasting & 2 & 735 & Risk Ratio (Random, 95\% Cl) & $0.64[0.19,2.18]$ \\
\hline $\begin{array}{l}5 \text { Moderate under- } \\
\text { weight }\end{array}$ & 2 & 2280 & Risk Ratio (Random, 95\% Cl) & $0.81[0.73,0.91]$ \\
\hline $\begin{array}{l}6 \text { Severe under- } \\
\text { weight }\end{array}$ & 1 & & Risk Ratio (Random, 95\% Cl) & Totals not selected \\
\hline 7 MUAC & 2 & 1512 & $\begin{array}{l}\text { Std. Mean Difference (IV, Random, 95\% } \\
\mathrm{Cl} \text { ) }\end{array}$ & $0.02[-0.08,0.12]$ \\
\hline 8 Haemoglobin $(\mathrm{g} / \mathrm{L})$ & 1 & 182 & Mean Difference (IV, Random, 95\% CI) & $0.29[-6.00,6.59]$ \\
\hline $9 \mathrm{HAZ}$ & 4 & 4047 & $\begin{array}{l}\text { Std. Mean Difference (IV, Random, 95\% } \\
\mathrm{CI} \text { ) }\end{array}$ & $0.06[0.00,0.13]$ \\
\hline
\end{tabular}




\begin{tabular}{lllll}
\hline $\begin{array}{l}\text { Outcome or sub- } \\
\text { group title }\end{array}$ & No. of studies & $\begin{array}{l}\text { No. of partici- } \\
\text { pants }\end{array}$ & Statistical method & Effect size \\
\hline $10 \mathrm{WAZ}$ & 3 & 1933 & Mean Difference (IV, Random, 95\% CI) & $0.05[-0.04,0.14]$ \\
\hline $11 \mathrm{WHZ}$ & 3 & 1933 & $\begin{array}{l}\text { Std. Mean Difference (IV, Random, 95\% } \\
\text { CI) }\end{array}$ & $0.07[-0.02,0.16]$ \\
\hline
\end{tabular}

\section{Analysis 5.1. Comparison 5 LNS versus fortified blended food (FBF), Outcome 1 Moderate stunting.}

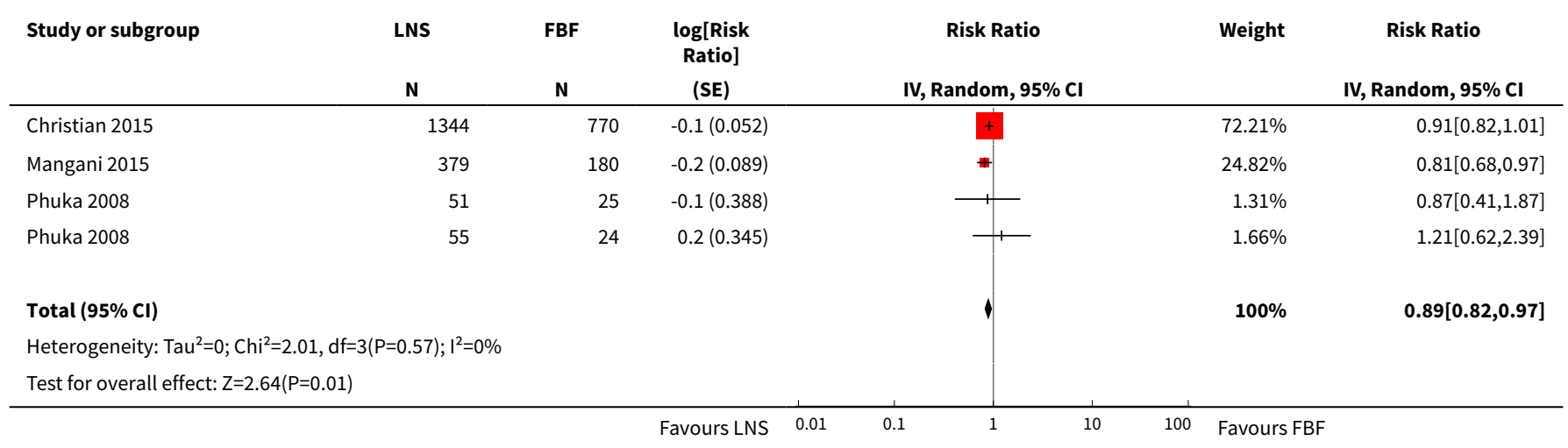

Analysis 5.2. Comparison 5 LNS versus fortified blended food (FBF), Outcome 2 Severe stunting.

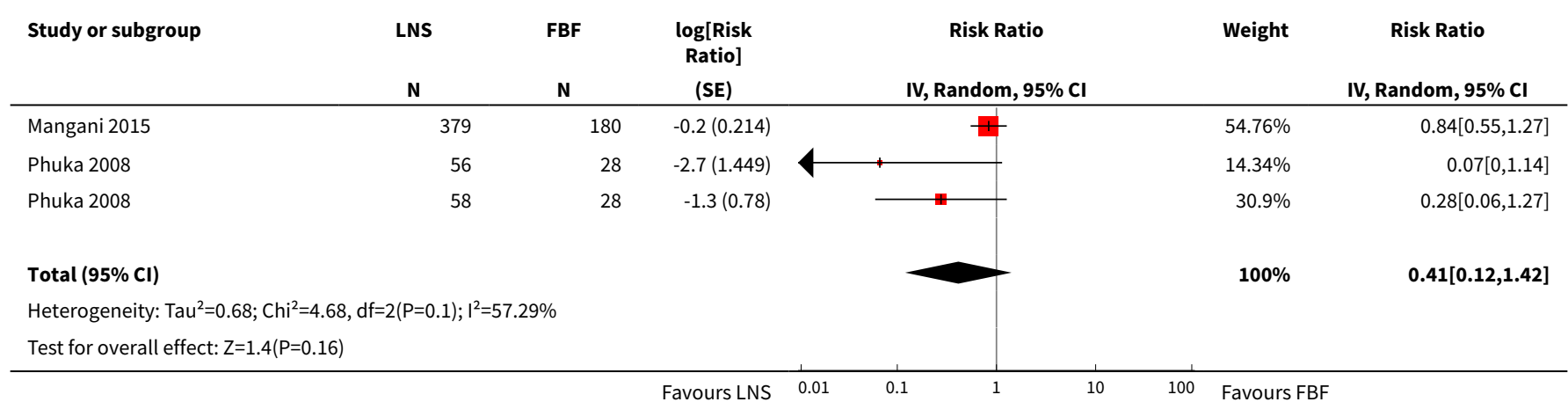

Analysis 5.3. Comparison 5 LNS versus fortified blended food (FBF), Outcome 3 Moderate wasting.

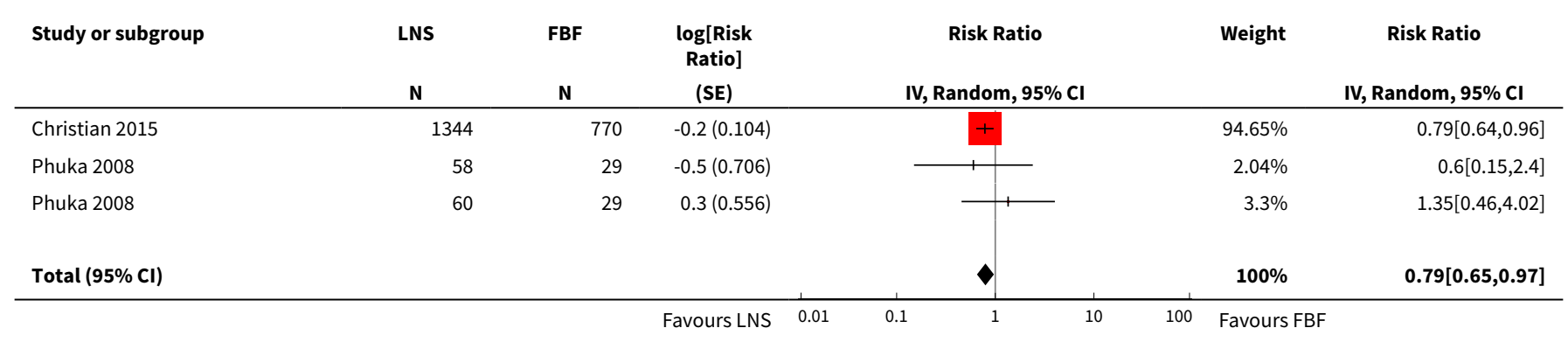




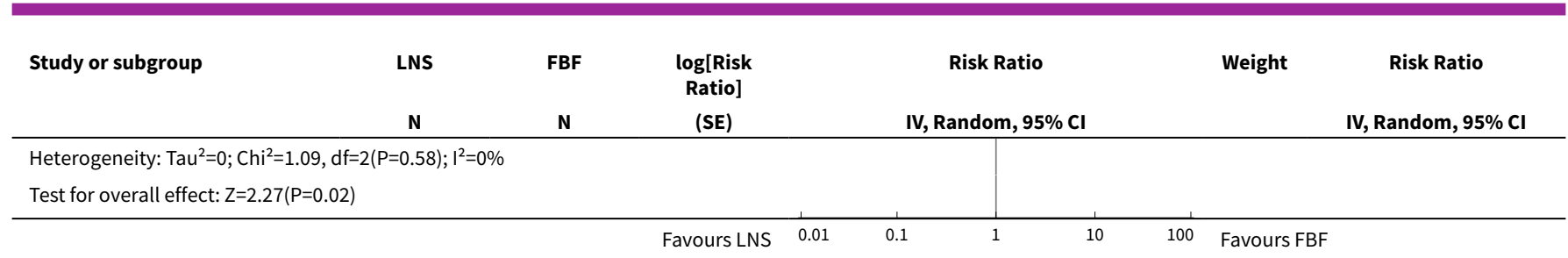

Analysis 5.4. Comparison 5 LNS versus fortified blended food (FBF), Outcome 4 Severe wasting.

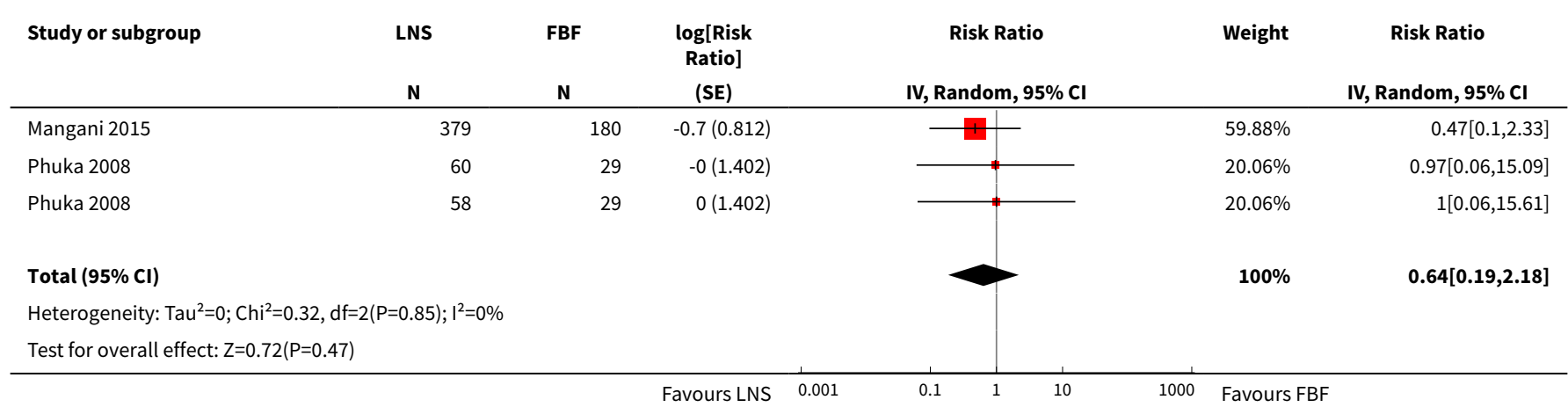

\section{Analysis 5.5. Comparison 5 LNS versus fortified blended food (FBF), Outcome 5 Moderate underweight.}

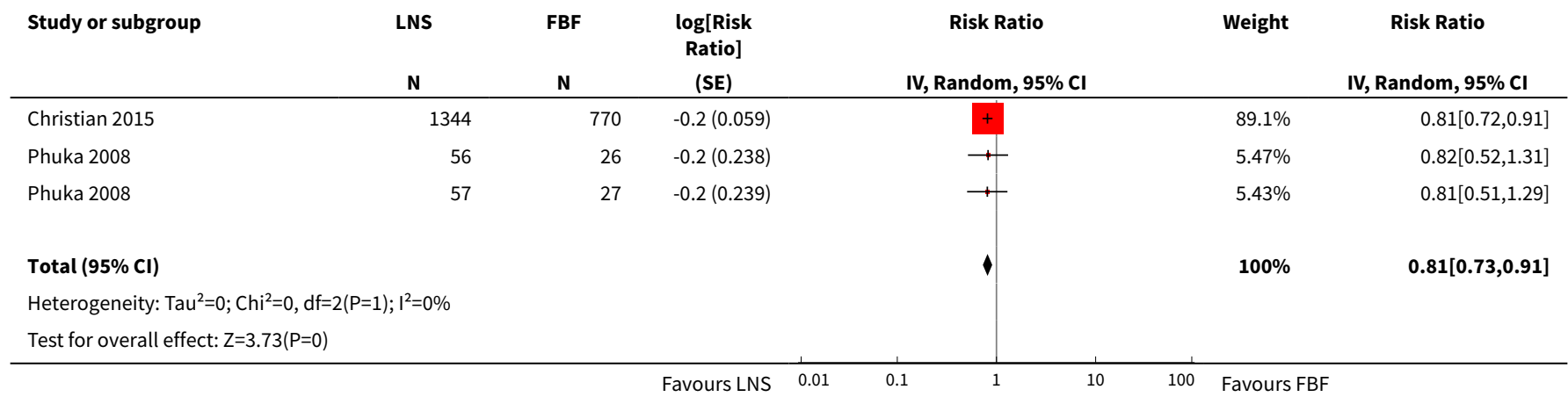

Analysis 5.6. Comparison 5 LNS versus fortified blended food (FBF), Outcome 6 Severe underweight.

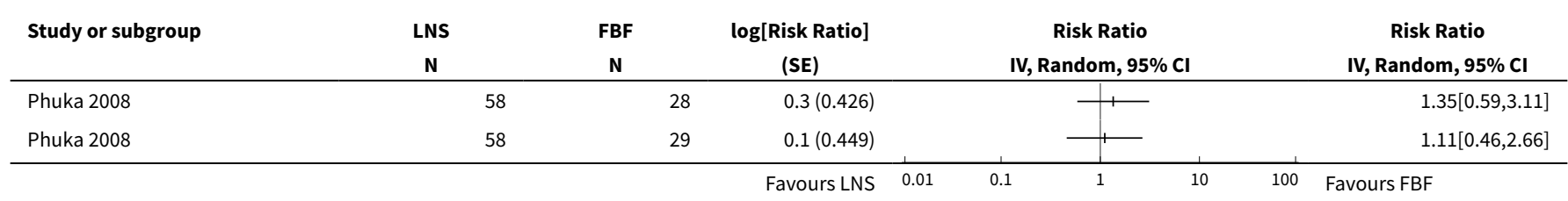


Analysis 5.7. Comparison 5 LNS versus fortified blended food (FBF), Outcome 7 MUAC.

\begin{tabular}{|c|c|c|c|c|c|c|c|}
\hline \multirow[t]{2}{*}{ Study or subgroup } & \multicolumn{2}{|c|}{ LNS } & \multicolumn{2}{|c|}{ FBF } & \multirow{2}{*}{$\begin{array}{c}\text { Std. Mean Difference } \\
\text { Random, } 95 \% \mathrm{Cl}\end{array}$} & \multirow[t]{2}{*}{ Weight } & \multirow{2}{*}{$\begin{array}{c}\text { Std. Mean Difference } \\
\text { Random, } 95 \% \mathrm{Cl}\end{array}$} \\
\hline & $\mathbf{N}$ & Mean(SD) & $\mathbf{N}$ & Mean(SD) & & & \\
\hline Bisimwa 2012 & 656 & $138.1(12)$ & 674 & $137.8(11.8)$ & & $89.13 \%$ & $0.03[-0.08,0.13]$ \\
\hline Phuka 2008 & 60 & $1(0.8)$ & 30 & $1.1(9)$ & & $5.36 \%$ & $-0.02[-0.46,0.42]$ \\
\hline Phuka 2008 & 61 & $1(1.1)$ & 31 & $1.1(9)$ & & $5.51 \%$ & $-0.02[-0.45,0.41]$ \\
\hline Total $* \star \star$ & 777 & & 735 & & & $100 \%$ & $0.02[-0.08,0.12]$ \\
\hline Test for overall effect & & & & & & & \\
\hline
\end{tabular}

Analysis 5.8. Comparison 5 LNS versus fortified blended food (FBF), Outcome 8 Haemoglobin (g/L).

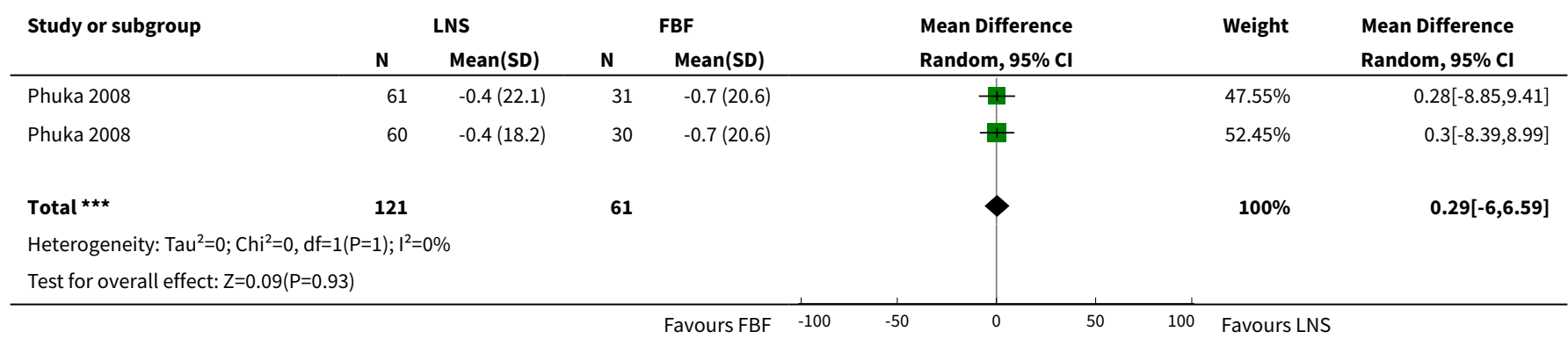

Analysis 5.9. Comparison 5 LNS versus fortified blended food (FBF), Outcome 9 HAZ.

\begin{tabular}{|c|c|c|c|c|c|c|c|}
\hline \multirow[t]{2}{*}{ Study or subgroup } & \multicolumn{2}{|c|}{ LNS } & \multicolumn{2}{|c|}{ FBF } & \multirow{2}{*}{$\begin{array}{c}\text { Std. Mean Difference } \\
\text { Random, } 95 \% \mathrm{Cl}\end{array}$} & \multirow[t]{2}{*}{ Weight } & \multirow{2}{*}{$\begin{array}{c}\text { Std. Mean Difference } \\
\text { Random, } 95 \% \mathrm{CI}\end{array}$} \\
\hline & $\mathbf{N}$ & $\operatorname{Mean}(S D)$ & $\mathbf{N}$ & Mean(SD) & & & \\
\hline Bisimwa 2012 & 656 & $-2(1.1)$ & 674 & $-2(1.2)$ & $\#$ & $34.54 \%$ & $0[-0.11,0.11]$ \\
\hline Christian 2015 & 1344 & $-1.8(1)$ & 770 & $-1.9(1)$ & + & $49.96 \%$ & $0.07[-0.02,0.16]$ \\
\hline Mangani 2015 & 212 & $-0.2(0.7)$ & 209 & $-0.4(0.7)$ & $\rightarrow$ & $11.15 \%$ & $0.22[0.03,0.41]$ \\
\hline Phuka 2008 & 61 & $-0.6(1.2)$ & 30 & $-0.7(1)$ & + & $2.16 \%$ & $0.13[-0.31,0.57]$ \\
\hline Phuka 2008 & 60 & $-0.6(0.9)$ & 31 & $-0.7(1)$ & -1 & $2.2 \%$ & $0.11[-0.32,0.55]$ \\
\hline Total $\star \star \star ~$ & 2333 & & 1714 & & 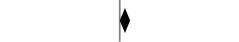 & $100 \%$ & $0.06[0,0.13]$ \\
\hline \multicolumn{8}{|c|}{ Heterogeneity: $\mathrm{Tau}^{2}=0 ; \mathrm{Chi}^{2}=4.07, \mathrm{df}=4(\mathrm{P}=0.4) ; \mathrm{I}^{2}=1.67 \%$} \\
\hline \multicolumn{3}{|c|}{ Test for overall effect: $Z=1.98(P=0.05)$} & & & & & \\
\hline
\end{tabular}

Analysis 5.10. Comparison 5 LNS versus fortified blended food (FBF), Outcome 10 WAZ.

\begin{tabular}{|c|c|c|c|c|c|c|c|}
\hline \multirow[t]{2}{*}{ Study or subgroup } & \multicolumn{2}{|c|}{ LNS } & \multicolumn{2}{|c|}{ FBF } & \multirow{2}{*}{$\begin{array}{l}\text { Mean Difference } \\
\text { Random, } 95 \% \mathrm{CI}\end{array}$} & \multirow[t]{2}{*}{ Weight } & \multirow{2}{*}{$\begin{array}{l}\text { Mean Difference } \\
\text { Random, } 95 \% \mathrm{CI}\end{array}$} \\
\hline & $\mathbf{N}$ & Mean(SD) & $\mathbf{N}$ & Mean(SD) & & & \\
\hline Bisimwa 2012 & 656 & $-1(1)$ & 674 & $-1(1)$ & + & $55.21 \%$ & $0[-0.11,0.11]$ \\
\hline Mangani 2015 & 212 & $-0.2(0.8)$ & 209 & $-0.4(0.9)$ & —- & $28.05 \%$ & $0.16[0,0.32]$ \\
\hline Phuka 2008 & 61 & $-1.2(0.9)$ & 30 & $-1.3(0.6)$ & + & $7.19 \%$ & $0.11[-0.21,0.43]$ \\
\hline
\end{tabular}




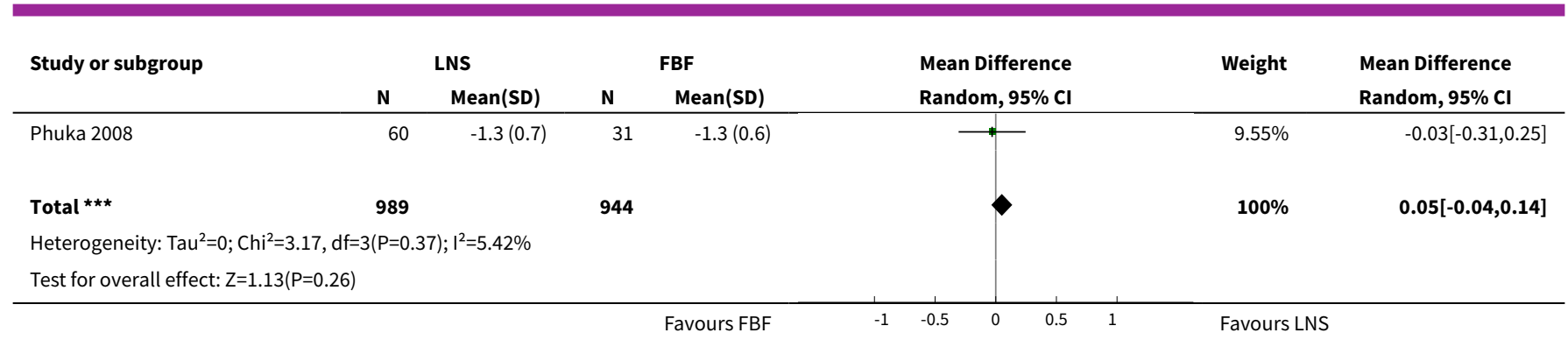

Analysis 5.11. Comparison 5 LNS versus fortified blended food (FBF), Outcome 11 WHZ.

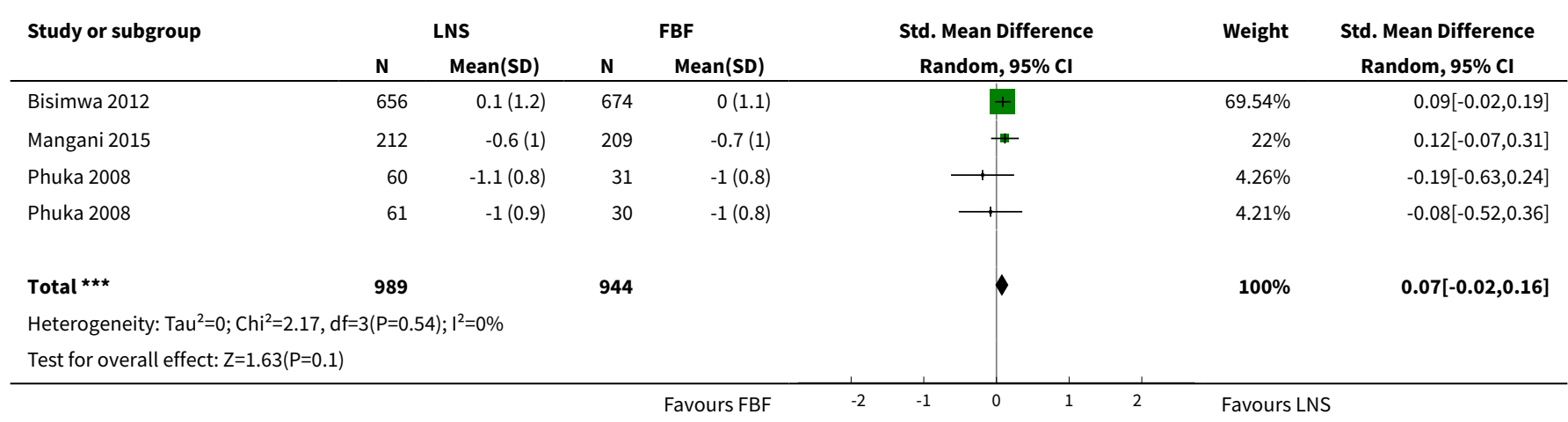

\section{Comparison 6. LNS versus micronutrient powders (MNP)}

\begin{tabular}{|c|c|c|c|c|}
\hline $\begin{array}{l}\text { Outcome or subgroup } \\
\text { title }\end{array}$ & No. of studies & $\begin{array}{l}\text { No. of partici- } \\
\text { pants }\end{array}$ & Statistical method & Effect size \\
\hline 1 Moderate stunting & 3 & 2365 & Risk Ratio (Random, 95\% Cl) & $0.92[0.82,1.02]$ \\
\hline 2 Moderate wasting & 2 & 2004 & Risk Ratio (Random, 95\% Cl) & $0.97[0.77,1.23]$ \\
\hline 3 Moderate underweight & 2 & 2004 & Risk Ratio (Random, 95\% Cl) & $0.88[0.78,0.99]$ \\
\hline 4 Anaemia & 2 & 557 & Risk Ratio (Random, 95\% Cl) & $0.38[0.21,0.68]$ \\
\hline $\begin{array}{l}5 \text { Anaemia: Sensitivity } \\
\text { analysis }\end{array}$ & 1 & & Risk Ratio (Random, 95\% Cl) & $0.56[0.27,1.14]$ \\
\hline $\begin{array}{l}6 \text { Serum haemoglobin }(\mathrm{g} / \\
\mathrm{L})\end{array}$ & 2 & 557 & Mean Difference (Random, 95\% Cl) & $5.13[2.00,8.26]$ \\
\hline $\begin{array}{l}7 \text { Serum haemoglobin (g/ } \\
\text { L): Sensitivity analysis }\end{array}$ & 1 & & Mean Difference (Random, 95\% Cl) & $3.6[-0.13,7.33]$ \\
\hline $8 \mathrm{HAZ}$ & 3 & 2362 & $\begin{array}{l}\text { Std. Mean Difference (IV, Random, 95\% } \\
\mathrm{CI} \text { ) }\end{array}$ & $0.10[-0.08,0.27]$ \\
\hline $\begin{array}{l}9 \text { HAZ: Sensitivity analy- } \\
\text { sis }\end{array}$ & 2 & 2001 & $\begin{array}{l}\text { Std. Mean Difference (IV, Random, 95\% } \\
\mathrm{CI} \text { ) }\end{array}$ & $0.16[0.03,0.28]$ \\
\hline
\end{tabular}




\begin{tabular}{lllll}
\hline $\begin{array}{l}\text { Outcome or subgroup } \\
\text { title }\end{array}$ & No. of studies & $\begin{array}{l}\text { No. of partici- } \\
\text { pants }\end{array}$ & Statistical method & Effect size \\
\hline 10 WAZ & 3 & 2362 & $\begin{array}{l}\text { Std. Mean Difference (IV, Random, 95\% } \\
\text { Cl) }\end{array}$ & $0.12[0.02,0.21]$ \\
\hline $\begin{array}{l}11 \text { WAZ: Sensitivity analy- } \\
\text { sis }\end{array}$ & 2 & 2001 & $\begin{array}{l}\text { Std. Mean Difference (IV, Random, 95\% } \\
\text { Cl) }\end{array}$ & $0.14[0.05,0.23]$ \\
\hline 12 WHZ & 3 & Std. Mean Difference (IV, Random, 95\% & $0.05[-0.06,0.17]$ \\
\hline $\begin{array}{l}13 \text { WHZ: Sensitivity } \\
\text { analysis }\end{array}$ & 2 & 2362 & Cl) & \\
\hline
\end{tabular}

Analysis 6.1. Comparison 6 LNS versus micronutrient powders (MNP), Outcome 1 Moderate stunting.

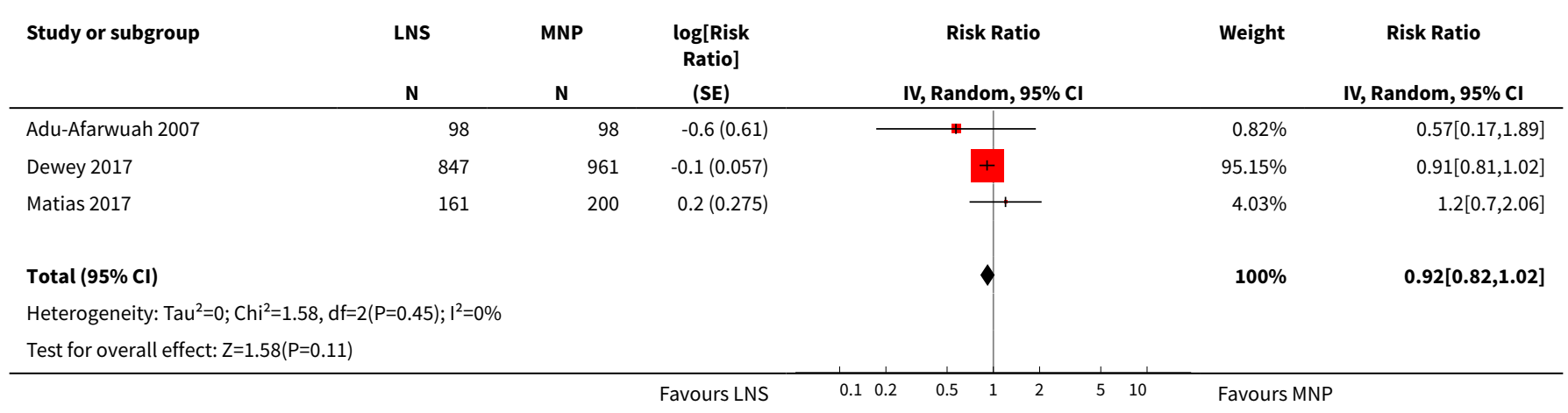

Analysis 6.2. Comparison 6 LNS versus micronutrient powders (MNP), Outcome 2 Moderate wasting.

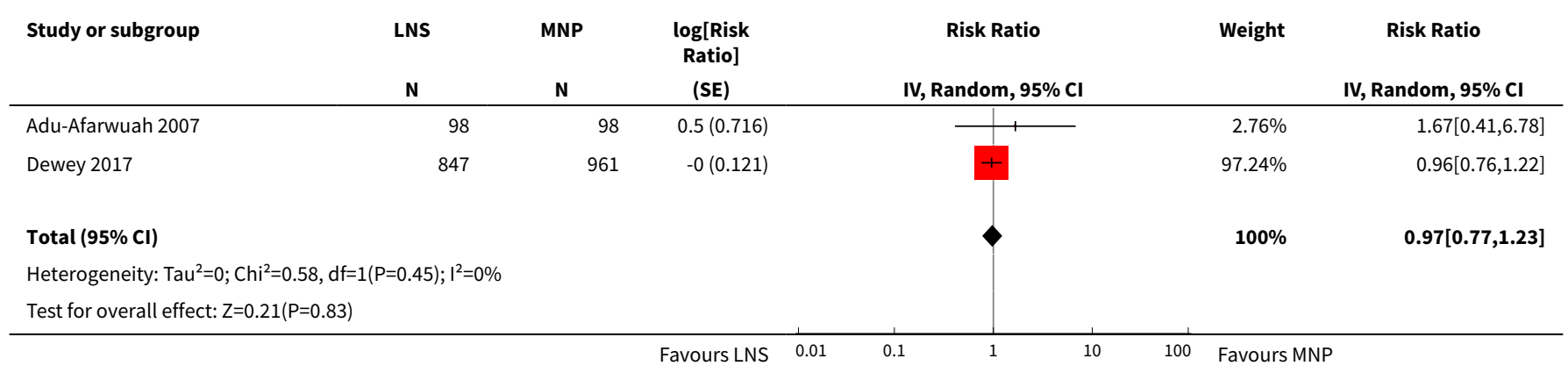

Analysis 6.3. Comparison 6 LNS versus micronutrient powders (MNP), Outcome 3 Moderate underweight.

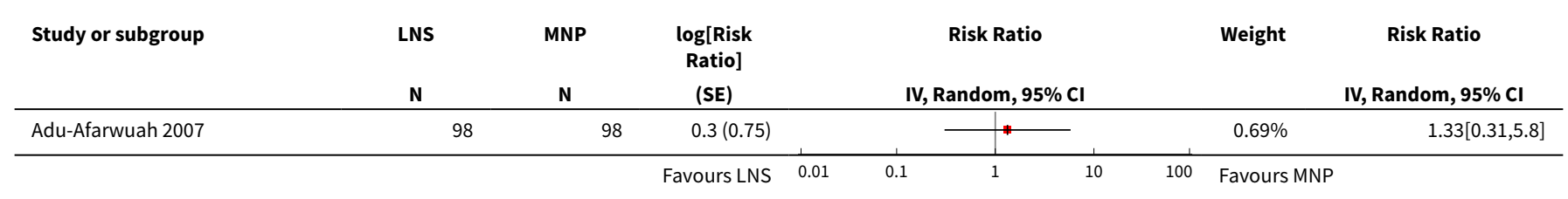




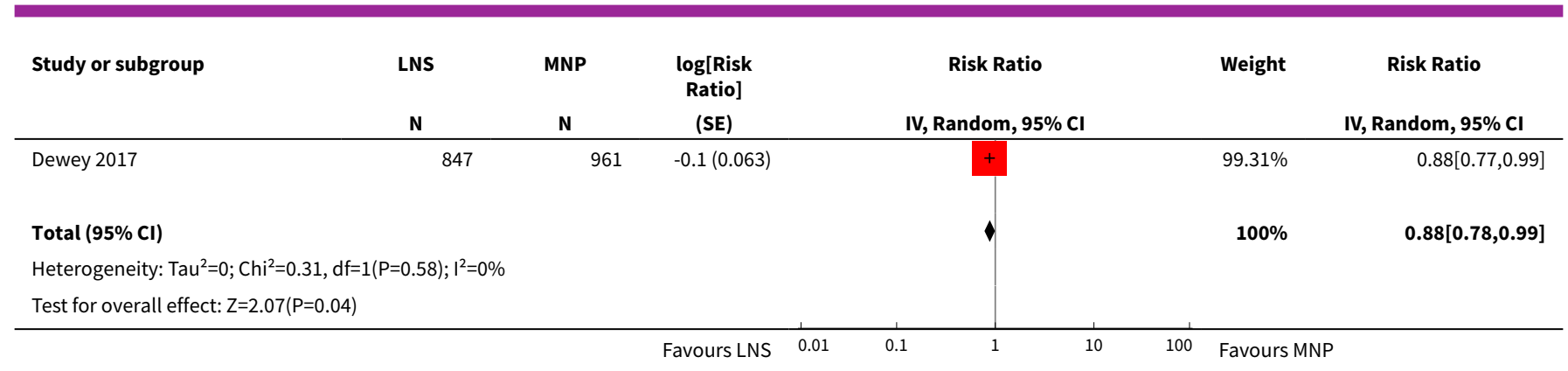

Analysis 6.4. Comparison 6 LNS versus micronutrient powders (MNP), Outcome 4 Anaemia.

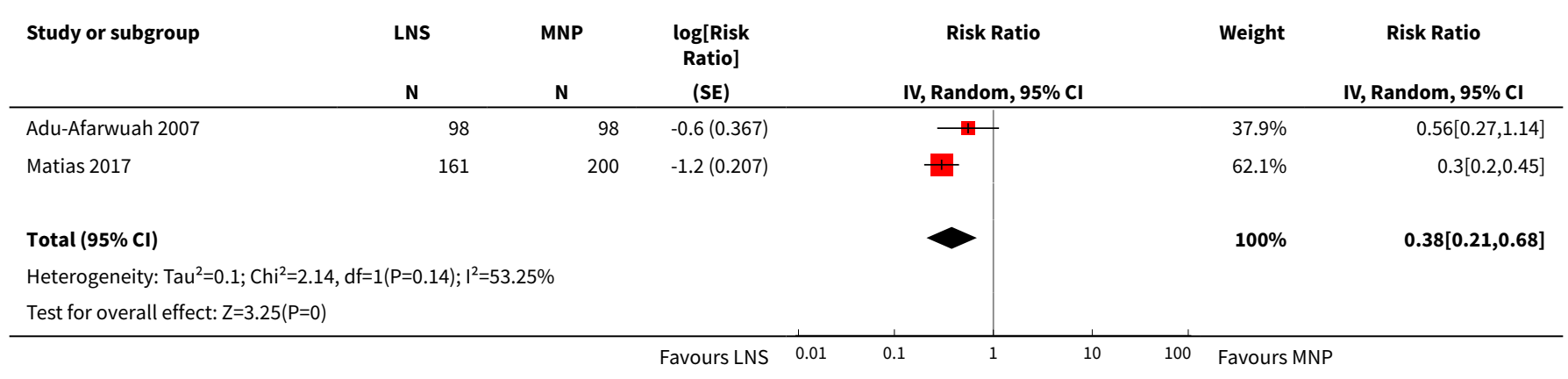

Analysis 6.5. Comparison 6 LNS versus micronutrient powders (MNP), Outcome 5 Anaemia: Sensitivity analysis.

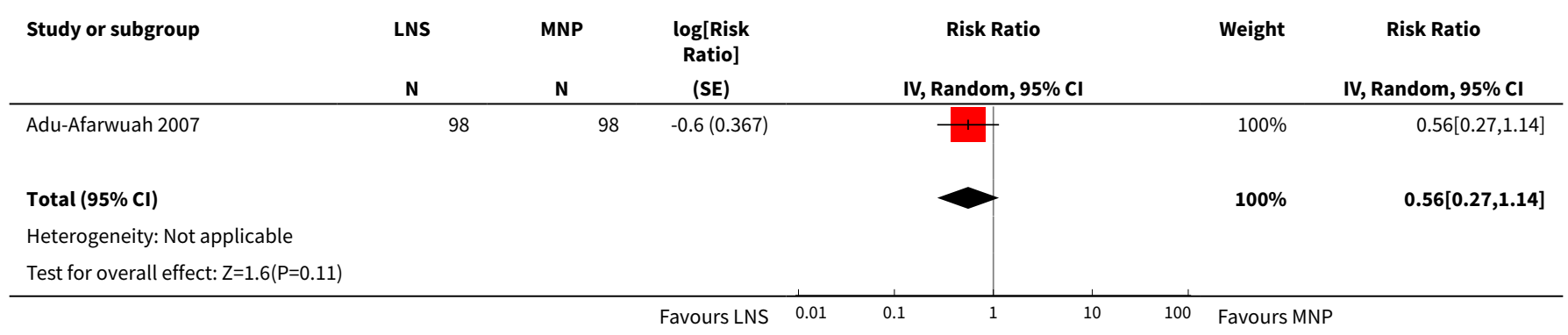

Analysis 6.6. Comparison 6 LNS versus micronutrient powders (MNP), Outcome 6 Serum haemoglobin (g/L).

\begin{tabular}{|c|c|c|c|c|c|c|}
\hline \multirow[t]{2}{*}{ Study or subgroup } & LNS & MNP & \multirow{2}{*}{$\begin{array}{l}\text { Mean Dif- } \\
\text { ference } \\
\text { (SE) }\end{array}$} & \multirow{2}{*}{$\begin{array}{c}\text { Mean Difference } \\
\text { IV, Random, } 95 \% \mathrm{CI}\end{array}$} & \multirow[t]{2}{*}{ Weight } & \multirow{2}{*}{$\begin{array}{c}\text { Mean Difference } \\
\text { IV, Random, } 95 \% \mathrm{Cl}\end{array}$} \\
\hline & $\mathbf{N}$ & $\mathbf{N}$ & & & & \\
\hline Adu-Afarwuah 2007 & 98 & 98 & $3.6(1.902)$ & 1 & $52.18 \%$ & $3.6[-0.13,7.33]$ \\
\hline Matias 2017 & 161 & 200 & $6.8(2.016)$ & & $47.82 \%$ & $6.8[2.85,10.75]$ \\
\hline Total $(95 \% \mathrm{Cl})$ & & & & & $100 \%$ & $5.13[2,8.26]$ \\
\hline Test for overall effect & & & & & & \\
\hline
\end{tabular}


Analysis 6.7. Comparison 6 LNS versus micronutrient powders

(MNP), Outcome 7 Serum haemoglobin (g/L): Sensitivity analysis.

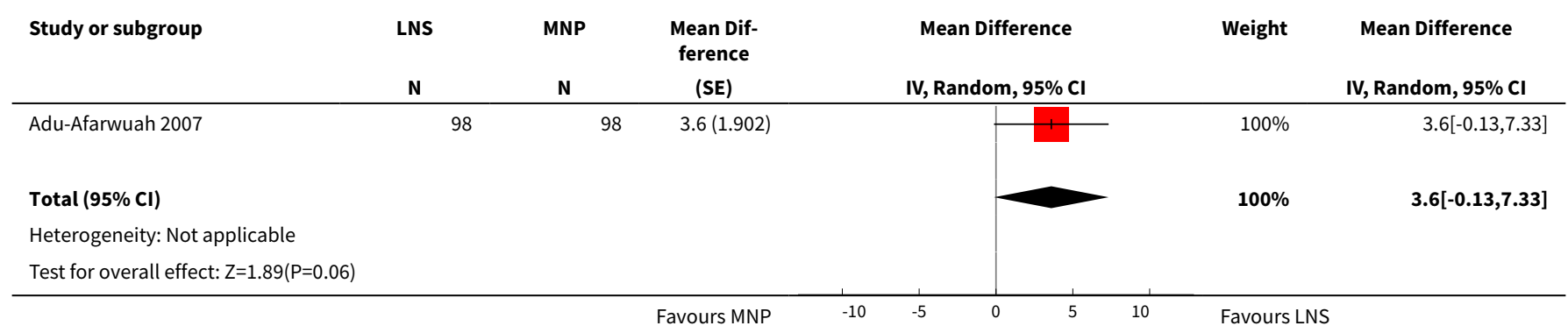

Analysis 6.8. Comparison 6 LNS versus micronutrient powders (MNP), Outcome 8 HAZ.

\begin{tabular}{|c|c|c|c|c|c|c|c|}
\hline \multirow[t]{2}{*}{ Study or subgroup } & \multicolumn{2}{|c|}{ LNS } & \multicolumn{2}{|c|}{ MNP } & \multirow{2}{*}{$\begin{array}{c}\text { Std. Mean Difference } \\
\text { Random, } 95 \% \mathrm{Cl}\end{array}$} & \multirow[t]{2}{*}{ Weight } & \multirow{2}{*}{$\begin{array}{c}\text { Std. Mean Difference } \\
\text { Random, } 95 \% \mathrm{Cl} \\
\end{array}$} \\
\hline & $\mathbf{N}$ & Mean(SD) & $\mathbf{N}$ & Mean(SD) & & & \\
\hline Adu-Afarwuah 2007 & 97 & $-0.2(0.5)$ & 96 & $-0.4(0.5)$ & - & $22.61 \%$ & $0.3[0.01,0.58]$ \\
\hline Dewey 2017 & 847 & $-1.7(1)$ & 961 & $-1.8(1)$ & + & $46.54 \%$ & $0.13[0.03,0.22]$ \\
\hline Matias 2017 & 161 & $-1.2(1)$ & 200 & $-1.1(1.1)$ & 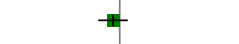 & $30.85 \%$ & $-0.09[-0.3,0.11]$ \\
\hline 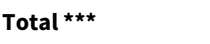 & 1105 & & 1257 & & 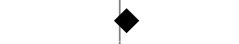 & $100 \%$ & $0.1[-0.08,0.27]$ \\
\hline Test for overall effect & & & & & & & \\
\hline
\end{tabular}

Analysis 6.9. Comparison 6 LNS versus micronutrient powders (MNP), Outcome 9 HAZ: Sensitivity analysis.

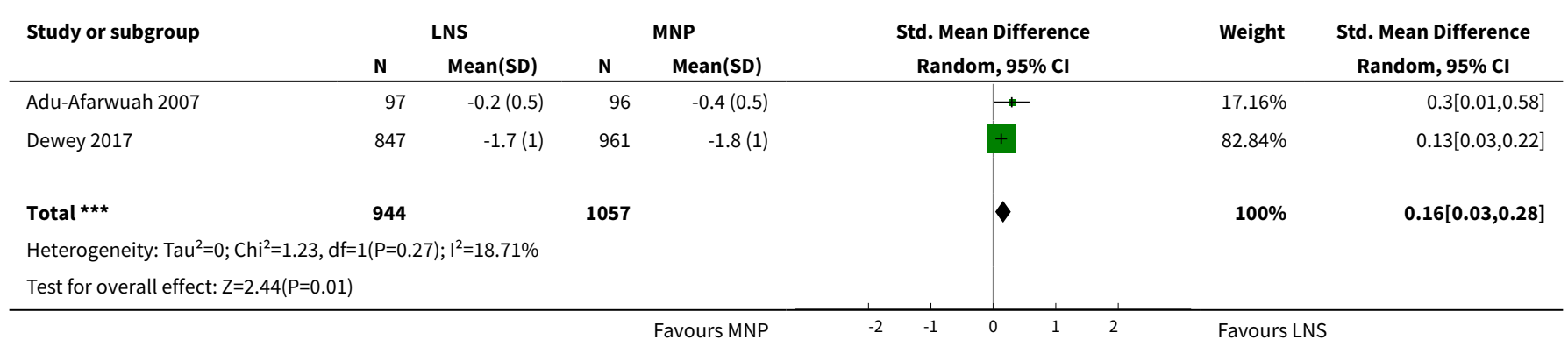

Analysis 6.10. Comparison 6 LNS versus micronutrient powders (MNP), Outcome 10 WAZ.

\begin{tabular}{|c|c|c|c|c|c|c|c|}
\hline \multirow[t]{2}{*}{ Study or subgroup } & \multicolumn{2}{|c|}{ LNS } & \multicolumn{2}{|c|}{ MNP } & \multirow{2}{*}{$\begin{array}{c}\text { Std. Mean Difference } \\
\text { Random, } 95 \% \mathrm{CI}\end{array}$} & \multirow[t]{2}{*}{ Weight } & \multirow{2}{*}{$\begin{array}{c}\text { Std. Mean Difference } \\
\text { Random, } 95 \% \mathrm{Cl}\end{array}$} \\
\hline & $\mathbf{N}$ & $\operatorname{Mean}(S D)$ & $\mathbf{N}$ & Mean(SD) & & & \\
\hline Adu-Afarwuah 2007 & 97 & $-0.5(0.5)$ & 96 & $-0.6(0.5)$ & + & $10.47 \%$ & $0.26[-0.03,0.54]$ \\
\hline Dewey 2017 & 847 & $-1.6(0.9)$ & 961 & $-1.8(0.9)$ & & $70.75 \%$ & $0.13[0.04,0.22]$ \\
\hline \multirow[t]{2}{*}{ Matias 2017} & 161 & $-0.2(0.9)$ & 200 & $-0.2(1)$ & + & $18.77 \%$ & $0[-0.21,0.21]$ \\
\hline & & & & avours MNP & $\begin{array}{ll}-2 & -1 \\
\end{array}$ & Favours L & \\
\hline
\end{tabular}




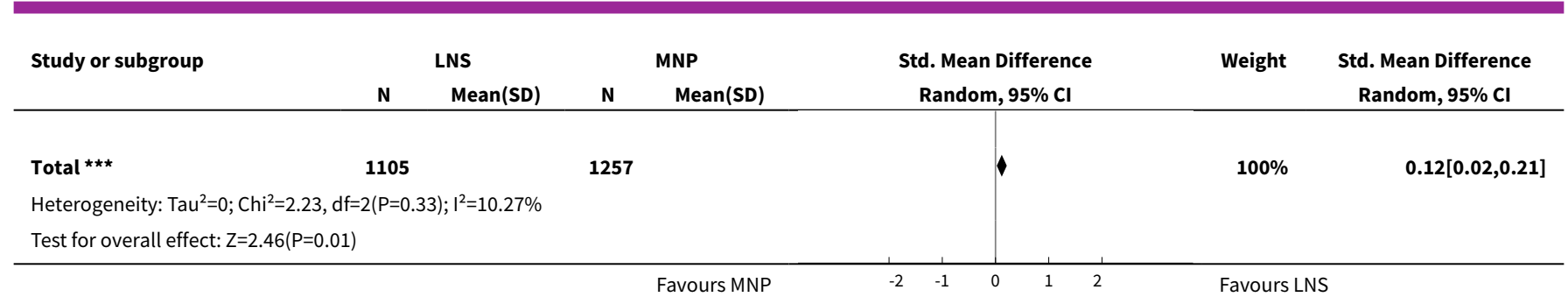

Analysis 6.11. Comparison 6 LNS versus micronutrient powders (MNP), Outcome 11 WAZ: Sensitivity analysis.

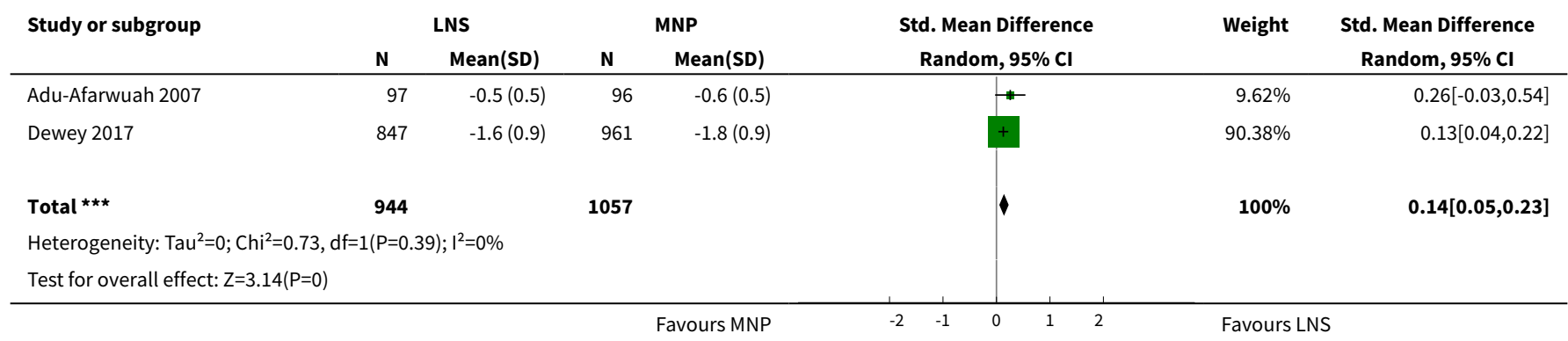

Analysis 6.12. Comparison 6 LNS versus micronutrient powders (MNP), Outcome 12 WHZ.

\begin{tabular}{|c|c|c|c|c|c|c|c|}
\hline \multirow[t]{2}{*}{ Study or subgroup } & \multicolumn{2}{|c|}{ LNS } & \multicolumn{2}{|c|}{ MNP } & \multirow{2}{*}{$\begin{array}{c}\text { Std. Mean Difference } \\
\text { Random, } 95 \% \mathrm{Cl}\end{array}$} & \multirow[t]{2}{*}{ Weight } & \multirow{2}{*}{$\begin{array}{c}\text { Std. Mean Difference } \\
\text { Random, } 95 \% \mathrm{Cl}\end{array}$} \\
\hline & $\mathbf{N}$ & Mean(SD) & $\mathbf{N}$ & $\operatorname{Mean}(S D)$ & & & \\
\hline Adu-Afarwuah 2007 & 97 & $-0.5(0.7)$ & 96 & $-0.6(0.7)$ & + & $14.61 \%$ & $0.14[-0.15,0.42]$ \\
\hline Dewey 2017 & 847 & $-1(0.9)$ & 961 & $-1.1(0.9)$ & + & $61.34 \%$ & $0.09[-0,0.18]$ \\
\hline Matias 2017 & 161 & $0.5(1)$ & 200 & $0.6(1.1)$ & * & $24.04 \%$ & $-0.09[-0.3,0.11]$ \\
\hline Total $\star \star \star ~$ & 1105 & & 1257 & & $\checkmark$ & $100 \%$ & $0.05[-0.06,0.17]$ \\
\hline \multicolumn{8}{|c|}{ Heterogeneity: $\mathrm{Tau}^{2}=0 ; \mathrm{Chi}^{2}=2.8, \mathrm{df}=2(\mathrm{P}=0.25) ; \mathrm{I}^{2}=28.69 \%$} \\
\hline \multicolumn{8}{|c|}{ Test for overall effect: $\mathrm{Z}=0.89(\mathrm{P}=0.38)$} \\
\hline
\end{tabular}

Analysis 6.13. Comparison 6 LNS versus micronutrient powders (MNP), Outcome 13 WHZ: Sensitivity analysis.

\begin{tabular}{|c|c|c|c|c|c|c|c|}
\hline \multirow[t]{2}{*}{ Study or subgroup } & \multicolumn{2}{|c|}{ LNS } & \multicolumn{2}{|c|}{ MNP } & \multirow{2}{*}{$\begin{array}{c}\text { Std. Mean Difference } \\
\text { Random, } 95 \% \mathrm{CI}\end{array}$} & \multirow[t]{2}{*}{ Weight } & \multirow{2}{*}{$\begin{array}{c}\text { Std. Mean Difference } \\
\text { Random, } 95 \% \mathrm{Cl}\end{array}$} \\
\hline & $\mathbf{N}$ & Mean(SD) & $\mathbf{N}$ & Mean(SD) & & & \\
\hline Adu-Afarwuah 2007 & 97 & $-0.5(0.7)$ & 96 & $-0.6(0.7)$ & + & $9.67 \%$ & $0.14[-0.15,0.42]$ \\
\hline Dewey 2017 & 847 & $-1(0.9)$ & 961 & $-1.1(0.9)$ & + & $90.33 \%$ & $0.09[-0,0.18]$ \\
\hline Total $\star \star \star$ & 944 & & 1057 & & $\checkmark$ & $100 \%$ & $0.1[0.01,0.18]$ \\
\hline \multicolumn{8}{|c|}{ Heterogeneity: $\mathrm{Tau}^{2}=0 ; \mathrm{Chi}^{2}=0.09, \mathrm{df}=1(\mathrm{P}=0.77) ; \mathrm{I}^{2}=0 \%$} \\
\hline \multicolumn{3}{|c|}{ Test for overall effect: $Z=2.12(P=0.03)$} & & & & & \\
\hline
\end{tabular}




\section{ADDITIONAL TABLES}

Table 1. Unused methods

\begin{tabular}{ll}
\hline Method & Approach \\
\hline $\begin{array}{l}\text { Measures of treatment ef- } \\
\text { fects }\end{array}$ & Rates \\
& $\begin{array}{l}\text { If rates represent events that could occur more than once per participant, we will report the rate } \\
\text { difference using the methodologies described in Deeks } 2011 .\end{array}$ \\
\end{tabular}

Unit of analysis issues

\section{Cluster-randomised trials}

We will follow the methods described in theCochrane Handbook for Systematic Reviews of Interventions (Higgins 2011b), and adjust the sample sizes or standard errors of cluster-randomised trials by using an estimate of the intra-cluster correlation co-efficient (ICC) derived from the study (if possible), from a similar trial or from a study of a similar population. If we use ICCs from other sources, we will report this in the results section, and conduct sensitivity analyses to investigate the effect of variation in the ICC.

We will acknowledge heterogeneity in the randomisation unit and perform a sensitivity analysis to investigate the effects of the randomisation unit in the future updates of the review, if applicable.

\section{Dealing with missing data}

\section{Assessment of reporting bias}

Subgroup analysis and investigation of heterogeneity
If we find studies with high levels of missing data, we will explore the effect in the overall assessment of treatment effect by removing such studies and conducting a sensitivity analysis.

\section{If we include 10 or more studies in a meta-analysis, we will investigate reporting biases (such as publication bias) using funnel plots. We will assess funnel plot asymmetry visually, and use formal tests for funnel plot asymmetry. For continuous outcomes, we will use the test proposed by Egger 1997. For dichotomous outcomes, we will use the test proposed by Harbord 2006. If asymmetry is detected in any of these tests or is suggested by a visual assessment, we will perform exploratory analyses to investigate it.}
We will conduct exploratory subgroup analyses on the primary outcomes, irrespective of hetero- geneity, when there are more than three studies contributing data. We will conduct the following analyses.

1. Breastfeeding practices (breastfed versus not breastfed)

2. Frequency of intervention (daily versus weekly versus flexible) 3. Living in an emergency-affected country (Wisner 2002), or in a refugee or internally displaced per-
sons' camp (yes verus no)

4. Anaemic status of participants at start of intervention (anaemic (defined as haemoglobin values $<110 \mathrm{~g} / \mathrm{L}$ ) versus non-anaemic or unknown status).

\section{Sensitivity analysis \\ We will carry out a sensitivity analysis to examine: \\ 1. the effect of removing non-randomised studies from the analysis; and \\ 2. the effects of different ICCS, and the randomisation unit, for cluster trials (if these are included).}

ICC: Intra-class correlation coefficient; WHO: World Health Organization.

Table 2. Composition of LNS

\begin{tabular}{lllll}
\hline Study & Total energy & Lipid content & Protein Content & Micronutrients \\
\hline $\begin{array}{llll}\text { Adu-Afarwuah } \\
2007\end{array}$ & $108 \mathrm{kcal}$ & $\begin{array}{l}\text { linoleic acid } \\
(1.29 \mathrm{~g}) ; \text { linolenic }\end{array}$ & Not specified & $\begin{array}{l}\text { Carotene }(400 \mu \mathrm{\mu g} \text { RE); vitamin C (30 mg); folic acid } \\
(80 \mathrm{~g}) ; \text { thiamine }(0.3 \mathrm{mg}) ; \text { riboflavin }(0.4 \mathrm{mg}) ; \text { vit- }\end{array}$ \\
& $\begin{array}{ll}\text { acid }(0.29 \mathrm{~g}) \\
\text { amin B3 }(4 \mathrm{mg}) ; \text { pantothenic acid }(1.8 \mathrm{mg}) ; \text { vita- }\end{array}$ & & min B6 $(0.3 \mathrm{mg}) ;$ vitamin B12 (0.5 g); iron sulphate \\
\hline
\end{tabular}

Preventive lipid-based nutrient supplements given with complementary foods to infants and young children 6 to 23 months of age for 
(9 mg); zinc sulphate (4 mg); calcium phosphate $(100 \mathrm{mg})$; potassium (152 mg); copper sulphate (0.2 $\mathrm{mg})$; sodium selenite (10 ug); potassium iodate (90 ug); phosphate (82 mg); magnesium (16 mg); manganese $(0.08 \mathrm{mg})$; phytate $(82 \mathrm{mg}$ )

\begin{tabular}{llll}
\hline $\begin{array}{l}\text { Adu-Afarwuah } \\
2016\end{array}$ & $\begin{array}{l}118 \mathrm{kcal}(20 \mathrm{~g} / \\
\text { day) }\end{array}$ & $9.6 \mathrm{~g}$ & $2.6 \mathrm{~g}$ \\
\hline
\end{tabular}

Linoleic acid (4.46 g); $\alpha$-linolenic acid $(0.58 \mathrm{~g})$; vit2016 day) amin A (400 $\mathrm{mg}$ retinol equivalents); thiamine (0.3 $\mathrm{mg})$; riboflavin (0.4 $\mathrm{mg})$; niacin $(4 \mathrm{mg})$; vitamin B6 (0.3 mg); vitamin B12 (0.5 mg); vitamin C (30 mg); vitamin D (5 mg); vitamin E (6 mg); vitamin K (30 $\mathrm{mg})$; folic acid ( $80 \mathrm{mg})$; pantothenic acid $(1.8 \mathrm{mg})$; iron (6 mg); zinc ( $8 \mathrm{mg}$ ); copper (0.34 mg); calcium (280 mg); phosphorus (190 mg); potassium (200 $\mathrm{mg}$ ); magnesium (40 mg); selenium (20 mg); iodine (90 mg); manganese (1.2 mg)

\begin{tabular}{llll}
\hline Ashorn 2015 & $\begin{array}{l}118 \mathrm{kcal}(20 \mathrm{~g} / \\
\text { day) }\end{array}$ & $9.6 \mathrm{~g}$ & $2.6 \mathrm{~g}$
\end{tabular}

$2.6 \mathrm{~g}$

(and

Linoleic acid (4.46 g); a-linolenic acid (0.58 g); vitamin A (400 mg RE); vitamin C (30 mg); vitamin B1 (0.3 mg); vitamin B2 (0.4 mg); niacin (4 mg); folic acid $(80 \mathrm{mg})$; pantothenic acid $(1.8 \mathrm{mg}) ;$ vitamin B6 (0.3 mg); vitamin B12 (0.5 mg); vitamin D (5 mg); vitamin $\mathrm{E}(6 \mathrm{mg})$; vitamin $\mathrm{K}(30 \mathrm{mg})$; iron (6 mg); zinc (8 mg); copper (0.34 mg); calcium (280 mg); phosphorus (190 mg); potassium (200 mg); magnesium (40 mg); selenium (20 mg); iodine $(90 \mathrm{mg})$; manganese $(1.2 \mathrm{mg})$

\begin{tabular}{ll}
\hline Bisimwa $2012 \quad \begin{array}{l}275 \mathrm{kcal}(50 \mathrm{~g} / \quad \text { Not specified } \\
\text { day) }\end{array}$ & Not specified
\end{tabular}

Vitamin A (412 IU); vitamin D (307 IU); vitamin C (75 $\mathrm{mg})$; thiamine $(0.7 \mathrm{mg})$; riboflavin $(1.0 \mathrm{mg})$; vitamin B12 $(1.0 \mu \mathrm{g})$; pyridoxine $(0.24 \mathrm{mg})$; niacin $(10.3 \mathrm{mg})$; pantothenic acid (3.8 mg); folic acid $(0.07 \mathrm{mg})$; vitamin $\mathrm{K}(0.01 \mathrm{mg})$; calcium (514 mg); phosphorus (265 mg); iron (9.5 mg); zinc (8.3 mg); copper (0.3 $\mathrm{mg})$; iodine $(0.07 \mathrm{mg})$; selenium $(0.003 \mathrm{mg})$; magnesium (23.4 mg); phytic acid $\left(0.425 \mathrm{mg}^{2}\right)$; phytic acid:iron molar ratio ${ }^{2}$ (2.1); phytic acid:zinc molar ratio $^{2}(1.9)$

\begin{tabular}{|c|c|c|c|c|}
\hline Christian 2015 & $\begin{array}{l}250 \mathrm{kcal}(46 \mathrm{~g} / \\
\text { day) }\end{array}$ & Not specified & Not specified & Not specified \\
\hline Dewey 2017 & $\begin{array}{l}118 \mathrm{kcal}(20 \mathrm{~g} / \\
\text { day) }\end{array}$ & $9.6 \mathrm{~g}$ & $2.6 \mathrm{~g}$ & 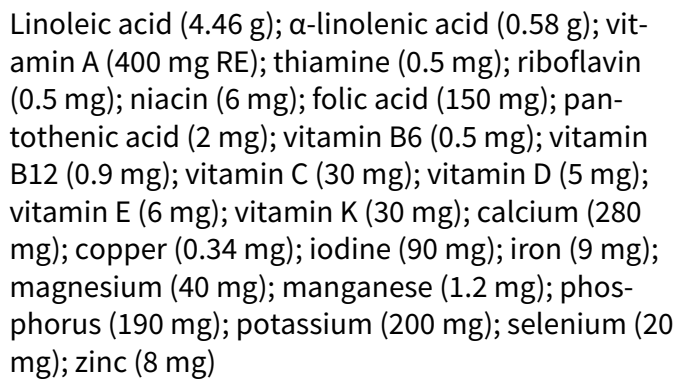 \\
\hline Hess 2015 & $\begin{array}{l}118 \mathrm{kcal}(20 \mathrm{~g} / \\
\text { day) }\end{array}$ & $9.6 \mathrm{~g}$ & $2.6 \mathrm{~g}$ & 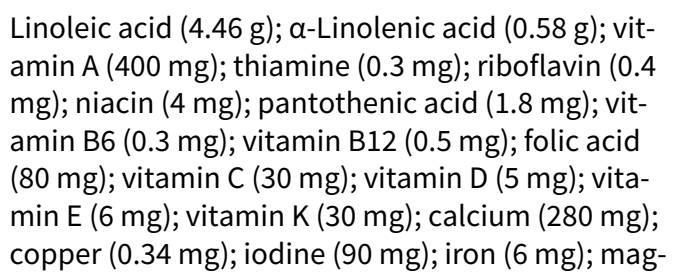 \\
\hline
\end{tabular}

Preventive lipid-based nutrient supplements given with complementary foods to infants and young children 6 to 23 months of age for health, nutrition, and developmental outcomes (Review)

Copyright @ 2019 The Cochrane Collaboration. Published by John Wiley \& Sons, Ltd. 
Table 2. Composition of LNS

(Continued)

nesium (40 mg); manganese (1.2 mg); phosphorus (190 mg); potassium (200 mg); selenium (20 $\mu \mathrm{g})$; zinc (0 mg)

\begin{tabular}{|c|c|}
\hline Huybregts 2012 & $247 \mathrm{kcal}$ (46 g/ \\
\hline
\end{tabular}

Linoleic acid ( $2 \mathrm{~g}$ ); $\alpha$-linolenic acid ( $0.3 \mathrm{~g})$; vitamin A (400 mg); vitamin E (6 mg); thiamine (0.5 mg); niacin (6 mg); pantothenic acid (2 mg); vitamin B6 (0.5 mg); folic acid (160 mg); vitamin B12 (0.9 mg); vitamin C (30 mg); magnesium (60 mg); zinc (4 mg); iron (9 mg); copper (0.3 mg); potassium (310 mg); calcium (387 mg); phosphorus (275 mg); selenium (17 mg); manganese $(0.17 \mathrm{mg})$; iodine $(90 \mathrm{mg}$ )

$\begin{array}{llll}\text { Iannotti } 2014 & \begin{array}{l}108 \mathrm{kcal}(20 \mathrm{~g} / \\ \text { day) }\end{array} & 7.08 \mathrm{~g} & 2.56 \mathrm{~g}\end{array}$

Linoleic acid (1.29 g); a-linoleic acid (0.29 g); vitamin A (400 mg); thiamine (0.3 mg); riboflavin (0.4 $\mathrm{mg})$; niacin (4 mg); pantothenic acid (1.8 $\mathrm{mg})$; vitamin B6 (0.3 mg); vitamin B12 (0.5 mg); folic acid (80 $\mathrm{mg})$; vitamin C (30 mg); calcium (100 mg); copper $(0.2 \mathrm{mg})$; iodine $(90 \mathrm{mg})$; iron $(9 \mathrm{mg})$; magnesium (16 mg); manganese (0.08 mg); phosphorus (82.2 $\mathrm{mg})$; potassium (152 mg); selenium (10 mg); zinc (4 $\mathrm{mg}$ )

$\begin{array}{llll}\text { Kumwenda } 2014 & \begin{array}{l}55 \mathrm{kcal}(10 \mathrm{~g} / \\ \text { day) }\end{array} & 4.7 \mathrm{~g} & 1.3 \mathrm{~g}\end{array}$
$.3 \mathrm{~g} \quad$ Linoleic acid (2.22 g); $\alpha$-linolenic acid (0.29 g); vit- amin A (400 $\mu \mathrm{g}$ RE); vitamin C (30 mg); vitamin B1 (0.3 mg); vitamin B2 (0.4 mg); niacin (4 mg); folic acid $(80 \mu \mathrm{g})$; pantothenic acid (1.8 $\mathrm{mg})$; vitamin B6 (0.3 mg); vitamin B12 (0.5 $\mathrm{\mu g})$; vitamin D (200 IU); vitamin E (6 $6 \mathrm{mg})$; vitamin $\mathrm{K}(30 \mu \mathrm{g})$; iron (6 mg); zinc ( $8 \mathrm{mg})$; copper (0.34 mg); calcium (240 mg); phosphorus (208 mg); potassium (265 mg); mag- nesium (50 mg); selenium (20 $\mu \mathrm{g})$; iodine (90 $\mu \mathrm{g})$; manganese $(1.2 \mathrm{mg})$; phytate $(28 \mathrm{mg}$ )

$17 \mathrm{kcal}(20 \mathrm{~g} / \quad 9.5 \mathrm{~g}$
day)

Linoleic acid (4.44 g); a-linolenic acid (0.58 g); vitamin A (400 $\mu \mathrm{g}$ RE); vitamin C (30 mg); vitamin B1 (0.3 mg); vitamin B2 (0.4 mg); niacin (4 mg); folic acid $(80 \mu \mathrm{g})$; pantothenic acid (1.8 $\mathrm{mg})$; vitamin B6 (0.3 mg); vitamin B12 (0.5 $\mathrm{mg})$; vitamin D (200 IU); vitamin $\mathrm{E}(66 \mathrm{mg})$; vitamin $\mathrm{K}(30 \mu \mathrm{g})$; iron $(6 \mathrm{mg})$; zinc (8 mg); copper (0.34 mg); calcium (240 mg); phosphorus (208 mg); potassium (265 mg); magnesium (50 mg); selenium (20 $\mu \mathrm{g})$; iodine $(90 \mu \mathrm{g})$; manganese (1.2 mg); phytate (56 mg)

\begin{tabular}{|c|c|c|c|}
\hline $\begin{array}{l}241 \text { kcal (40 g/ } \\
\text { day) }\end{array}$ & $18.9 \mathrm{~g}$ & $5 \mathrm{~g}$ & 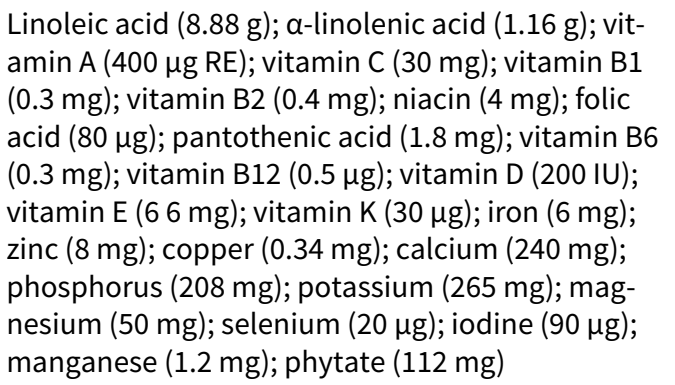 \\
\hline $\begin{array}{l}118 \mathrm{kcal}(20 \mathrm{~g} / \\
\text { day) }\end{array}$ & $9.6 \mathrm{~g}$ & $2.6 \mathrm{~g}$ & $\begin{array}{l}\text { Linoleic acid (4.46 g); Alpha-linolenic acid (0.58 g); } \\
\text { Vitamin A }(400 \mu \mathrm{g}) \text {; Vitamin D }(5 \mu \mathrm{g}) \text {; Vitamin E ( } 6 \\
\mathrm{mg}) \text {; Vitamin K ( } 30 \mu \mathrm{g}) \text {; Vitamin C (30 mg) Folic acid } \\
(150 \mu \mathrm{g}) \text {; Thiamine (B1) (0.5 mg); Riboflavin (B2) }\end{array}$ \\
\hline
\end{tabular}

Preventive lipid-based nutrient supplements given with complementary foods to infants and young children 6 to 23 months of age for 
(0.5 mg); Niacin (6 mg); Pantothenic acid (B5) (2 $\mathrm{mg})$; Vitamin B6 (0.5 mg); Vitamin B12 $(0.9 \mu \mathrm{g})$; Calcium (280 mg); Copper (0.34 mg); lodine (90 $\mu \mathrm{g})$; Iron (9 mg); Magnesium (40 mg); Manganese (1.2 $\mathrm{mg}$ ); Phosphorous (190 mg); Potassium (200 mg); Selenium $(20 \mu \mathrm{g})$; Zinc (8 mg)

\begin{tabular}{lll}
\hline Mangani 2015 & Milk-LNS $\quad 17.9 \mathrm{~g}$ & $8.2 \mathrm{~g}$ \\
& $\begin{array}{l}284.8 \mathrm{kcal}(54 \mathrm{~g} / \\
\text { day) }\end{array}$
\end{tabular}

Retinol (400 $\mu \mathrm{g} \mathrm{RE})$; folate $(160 \mu \mathrm{g})$; niacin $(6 \mathrm{mg})$; pantothenic acid (2 mg); riboflavin (0.5 mg); thiamine $(0.5 \mathrm{mg})$; vitamin B6 $(0.5 \mathrm{mg})$; vitamin B12 $(0.9 \mu \mathrm{g})$; vitamin C (30 mg); vitamin D $(5 \mu \mathrm{g})$; calcium (366 mg); copper (0.4 mg); iodine $(90 \mu \mathrm{g})$; iron (6 mg); magnesium (78.5 mg); selenium (20 $\mu \mathrm{g})$; zinc (6.0 mg); phosphorus (185.6 mg); potassium (318.6 mg); manganese (0.60 mg)

Soy-LNS $18.5 \mathrm{~g}$
$\begin{aligned} & 276.1 \mathrm{kcal}(54 \mathrm{~g} / \\ & \text { day) }\end{aligned}$

Retinol (400 $\mu \mathrm{g} \mathrm{RE})$; folate $(160 \mu \mathrm{g})$; niacin (6 mg); pantothenic acid ( $2 \mathrm{mg})$; riboflavin $(0.5 \mathrm{mg})$; thiamine $(0.5 \mathrm{mg})$; vitamin B6 (0.5 mg); vitamin B12 $(0.9 \mu \mathrm{g})$; vitamin C (30 mg); vitamin D $(5 \mu \mathrm{g})$; calcium (366 mg); copper $(0.4 \mathrm{mg})$; iodine $(90 \mu \mathrm{g})$; iron (6 mg); magnesium (78.5 mg); selenium (20 $\mu \mathrm{g})$; zinc $(6.0 \mathrm{mg})$; phosphorus (185.6 $\mathrm{mg})$; potassium (307.3 mg); manganese (0.60 mg)

$\begin{array}{ll}\text { Matias } 2017 & \begin{array}{l}110 \mathrm{kcal}(20 \mathrm{~g} / \\ \text { day })\end{array}\end{array}$

Linoleic acid (1.29 g); $\alpha$-linolenic acid (0.29 g); folic acid $(80 \mu \mathrm{g})$; niacin $(4 \mathrm{mg})$; pantothenic acid (1.8 $\mathrm{mg}$ ); riboflavin $(0.4 \mathrm{mg})$; thiamine $(0.3 \mathrm{mg})$; vitamin A $(400 \mu \mathrm{g})$; vitamin B12 $(0.5 \mu \mathrm{g})$; vitamin B6 (0.3 $\mathrm{mg}$ ); vitamin C (30 mg); calcium (100 mg); copper $(0.2 \mathrm{mg})$; iodine $(90 \mu \mathrm{g})$; iron $(9 \mathrm{mg})$; magnesium (16 $\mathrm{mg})$; manganese $(0,08 \mathrm{mg})$; phosphorous ( $82 \mathrm{mg})$; potassium (152 mg); selenium (10 $\mu \mathrm{g})$; zinc (4 mg)

\begin{tabular}{|c|c|c|}
\hline Null 2018 & $\begin{array}{l}118 \mathrm{kcal}(20 \mathrm{~g} / \\
\text { day) }\end{array}$ & $9.6 \mathrm{~g}$ \\
\hline
\end{tabular}

Linoleic acid (4.46 g); Alpha-linolenic acid (0.58 g); Vitamin A (400 $\mu \mathrm{g})$; Vitamin D (5 $\mu \mathrm{g})$; Vitamin E (6 $\mathrm{mg})$; Vitamin K (30 $\mu \mathrm{g})$; Vitamin C (30 mg) Folic acid $(150 \mu \mathrm{g})$; Thiamine (B1) $(0.5 \mathrm{mg})$; Riboflavin (B2) (0.5 mg); Niacin (6 mg); Pantothenic acid (B5) (2 $\mathrm{mg})$; Vitamin B6 (0.5 mg); Vitamin B12 $(0.9 \mu \mathrm{g})$; Calcium (280 mg); Copper (0.34 mg); lodine (90 $\mu \mathrm{g})$; Iron (9 mg); Magnesium (40 mg); Manganese (1.2 $\mathrm{mg}$ ); Phosphorous (190 mg); Potassium (200 mg); Selenium $(20 \mu \mathrm{g})$; Zinc (8 mg)

\begin{tabular}{llll}
\hline Olney 2018 & $\begin{array}{l}118 \mathrm{kcal}(20 \mathrm{~g} / \\
\text { day) }\end{array}$ & $9.6 \mathrm{~g}$ & $2.6 \mathrm{~g}$
\end{tabular}

Linoleic acid (4.5 g); $\alpha$-linolenic acid ( $0.6 \mathrm{~g})$; vitamin A (400 $\mu \mathrm{g})$; vitamin C (30 mg); vitamin D (5 mg); vitamin $\mathrm{E}(6 \mathrm{mg})$; vitamin $\mathrm{K}(30 \mathrm{mg})$; thiamine $(0.5$ $\mathrm{mg}$ ); riboflavin (0.5 mg); niacin (6 mg); pantothenic acid (2 mg); vitamin B6 (0.5 mg); folic acid (150 $\mu \mathrm{g})$; vitamin B12 $(0.9 \mu \mathrm{g})$; iron $(9 \mathrm{mg})$; zinc $(8 \mathrm{mg})$; copper $(0.3 \mathrm{mg})$; selenium $(20 \mu \mathrm{g})$; iodine $(90 \mu \mathrm{g})$; calcium (280 mg); magnesium (40 mg); manganese (1.2 $\mathrm{mg}$ ); phosphorus (190 mg); potassium (200 mg)

$\begin{array}{llll}\text { Phuka } 2008 & \begin{array}{l}130 \mathrm{kcal}(25 \mathrm{~g} / \\ \text { day) }\end{array} & 8.3 \mathrm{~g} & 3.8 \mathrm{~g}\end{array}$

Retinol (400 mg RE); folate (160 mg); niacin (6 mg); pantothenic acid ( $2 \mathrm{mg})$; riboflavin $(0.5 \mathrm{mg})$; thiamine (0.5 mg); vitamin B 6 (0.5 mg); vitamin B12 $(0.9 \mathrm{mg})$; vitamin C (30 mg); vitamin D (5 mg); calcium (283 mg); copper (0.5 mg); iodine (90 mg); iron 
Table 2. Composition of LNS

(8 mg); magnesium (60 mg); selenium (17 mg); zinc (8.4 mg)

$\begin{array}{lll}264 \mathrm{kcal}(50 \mathrm{~g} / & 16.5 \mathrm{~g} & 7.6 \mathrm{~g}\end{array}$

Retinol (400 mg RE); folate (160 mg); niacin (6 mg); pantothenic acid ( $2 \mathrm{mg})$; riboflavin $(0.5 \mathrm{mg})$; thiamine $(0.5 \mathrm{mg})$; vitamin B6 (0.5 mg); vitamin B12 (0.9 mg); vitamin C (30 mg); vitamin D (5 mg); calcium (366 mg); copper (0.4 mg); iodine (90 mg); iron (8 mg); magnesium (60 mg); selenium (17 mg); zinc (8.4 mg)

\begin{tabular}{|c|c|c|c|c|}
\hline Siega-Riz 2014 & $\begin{array}{l}247 \mathrm{kcal}(46.4 \mathrm{~g} / \\
\text { day) }\end{array}$ & $16 \mathrm{~g}$ & $5.9 \mathrm{~g}$ & $\begin{array}{l}\text { Vitamin A ( } 400 \mu \mathrm{g}) \text {; vitamin B12 }(0.9 \mu \mathrm{g}) \text {; iron (9 } \\
\mathrm{mg}) ; \text { zinc }(9 \mathrm{mg})\end{array}$ \\
\hline
\end{tabular}

g: gram;

LNS: lipid-based nutrient supplements

mg: milligram

\section{AP PE N DICES}

\section{Appendix 1. Search strategies for retracted studies}

\section{MEDLINE Ovid 1946 to February Week 42019}

Searched 5 March 2019. 45 records were retrieved, none of which related to included studies in this review.

\section{1 exp Lipids/}

2 fatty acid\$.tw,kf.

3 Docosahexaenoic acid.tw,kf.

4 Eicosapentaenoic Acid\$.tw,kf.

5 PUFAS.tw,kf.

6 lipid.tw,kf.

7 (omega $3 \$$ or omega $6 \$$ ).tw,kf.

8 (soy\$ or peanut or groundnut or whey or sesame or cashew or chickpea or oil\$).tw,kf.

9 or/1-8

10 Dietary Supplements/

11 Food, fortified/

12 ((diet\$ or food\$) adj3 (fortif\$ or enrich\$ or supplement\$)).tw,kf.

13 (complement\$ adj3 (food\$ or feed\$)).tw,kf.

14 "Ready to use".tw,kf.

15 (RUSF or RUTF).tw,kf.

16 "point of use".tw,kf.)

17 (home\$ adj2 fortif\$).tw,kf.

18 or $/ 10-17$

199 and 18

20 (lipid\$ adj3 nutrient\$).tw,kf.

21 (lipid\$ adj3 supplement\$).tw,kf.

22 lipid based.tw,kf.

23 (lipid\$ adj3 fortif\$).tw,kf.

24 (lipid\$ adj3 enrich\$).tw,kf.

25 (lipid\$ adj2 emuls\$).tw,kf.

26 (lipid\$ adj2 formulation\$).tw,kf.

27 (Lipid\$ adj3 powder\$).tw,kf.

28 (lipid adj3 spread\$).tw,kf.

29 (lipid\$ adj3 paste\$).tw,kf.

30 (Nutributter\$ or Plumpy\$).tw,kf.

31 (LBNS\$ or LNS\$1 or iLiNS).tw,kf.

32 or/20-31

3319 or 32 (43056)

Preventive lipid-based nutrient supplements given with complementary foods to infants and young children 6 to 23 months of age for health, nutrition, and developmental outcomes (Review)

Copyright $\odot 2019$ The Cochrane Collaboration. Published by John Wiley \& Sons, Ltd. 
34 Infant/ (753966)

35 (baby or babies or infant\$ or toddler\$ or child\$).tw. (1425823)

3634 or 35 (1743645)

3733 and 36 (3151)

38 exp animals/ not humans.sh.

3937 not 38

40 (comment or "corrected and republished article" or editorial or "expression of concern" or published erratum or retracted publication or "retraction of publication").pt.

41 (comment or corrected or correction or corrigendum or "expression of concern" or erratum or retracted or retraction).ti.

4240 or 41

4339 and 42

\section{Embase Ovid 1974 to 2019 March 04}

Searched 5 March 2019. records were retreived, none of which related to included studies in this review.

1 exp Lipids/

2 fatty acid\$.tw,kw.

3 Docosahexaenoic acid.tw,kw.

4 Eicosapentaenoic Acid\$.tw,kw.

5 PUFA\$.tw,kw.

6 lipid\$.tw,kw.

7 (omega $3 \$$ or omega $6 \$$ ).tw,kw.

8 (soy\$ or peanut or groundnut or whey or sesame or cashew or chickpea or oil\$).tw,kw.

9 or/1-8

10 dietary supplement/

11 fortified food/

12 ((diet\$ or food\$) adj3 (fortif\$ or enrich\$ or supplement\$)).tw,kw.

13 (complement\$ adj3 (food\$ or feed\$)).tw,kw.

14 "Ready to use".tw,kw.

15 (RUSF or RUTF).tw,kw.

16 "point of use".tw,kw.

17 (home\$ adj2 fortif\$).tw,kw.

18 or/10-17

199 and 18

20 lipid based.tw,kw.

21 (lipid\$ adj3 nutrient\$).tw,kw.

22 (lipid\$ adj3 supplement\$).tw,kw.

23 (lipid\$ adj3 fortif\$).tw,kw.

24 (lipid\$ adj3 enrich\$).tw,kw.

25 (lipid\$ adj2 emuls\$).tw,kw.

26 (lipid\$ adj2 formulation\$).tw,kw.

27 (Lipid\$ adj3 powder\$).tw,kw.

28 (lipid adj3 spread\$).tw,kw.

29 (lipid\$ adj3 paste\$).tw,kw.

30 (Nutributter\$ or Plumpy\$).tw,kw.

31 (LBNS\$ or LNS\$1 or iLiNS).tw,kw.

32 or $/ 20-31$

3319 or 32

34 infant/

35 (baby or babies or infant\$ or toddler\$ or child\$).tw.

3634 or 35

3733 and 36

38 exp animals/ or exp invertebrate/ or animal experiment/ or animal model/ or animal tissue/ or animal cell/ or nonhuman/)

39 human/ or normal human/ or human cell/

4038 not 39

4137 not 40

42 exp erratum/

43 erratum.pt.

44 yes.nr.

45 tombstone.pt.

46 (comment or corrected or correction or corrigendum or "expression of concern" or erratum or retracted or retraction).ti.

47 or $/ 42-46$

Preventive lipid-based nutrient supplements given with complementary foods to infants and young children 6 to 23 months of age for health, nutrition, and developmental outcomes (Review)

Copyright (c) 2019 The Cochrane Collaboration. Published by John Wiley \& Sons, Ltd. 
4841 and 47

\section{Retraction Watch Database retractiondatabase.org/RetractionSearch.aspx?}

Searched 5 March 2019.

We searched for retractions of all reports of included studies using PubMed ID (where a PubMed ID existed for the report), and ran separate searches for the title of each paper. When searching by title alone retrieved a large number of records, we searched by a combination of title and surname of first author.

\section{Appendix 2. Search strategies for intervention studies}

\section{Cochrane Central Register of Controlled Trials (CENTRAL) in the Cochrane Library}

$\# 1$ [mh Lipids]

\#2(fatty next acid*)

\#3((Docosahexaenoic or Eicosapentaenoic) next acid $\left.{ }^{\star}\right)$

\#4(PUFA or PUFAs)

\#5lipid*

\#6(omega next $\left(3^{\star}\right.$ or $\left.6^{\star}\right)$ )

$\# 7$ (soy ${ }^{\star}$ or peanut or groundnut or whey or sesame or cashew or chickpea or oil ${ }^{\star}$ )

\#8\{or \#1-\#7\}

\#9[mh "Dietary Supplements"]

$\# 10$ [mh "Food, fortified"]

$\# 11\left(\left(\right.\right.$ diet $^{\star}$ or food $\left.^{\star}\right)$ near/3 (fortif* or enrich $^{\star}$ or supplement $\left.{ }^{\star}\right)$ )

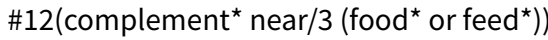

\#13"Ready to use"

\#14"point of use"

\#15(RUSF or RUTF)

$\# 16$ (home $^{\star}$ near/2 fortif ${ }^{\star}$ )

\#17\{0r \#9-\#16\}

$\# 18 \# 8$ and \#17

\#19(lipid next based)

\#20(lipid* near/3 supplement ${ }^{\star}$ )

\#21(lipid* near/3 nutrient* ${ }^{\star}$

\#22 (lipid* near/3 fortif*)

\#23(lipid* near/3 formulation ${ }^{\star}$ )

\#24(lipid* near/3 enrich ${ }^{\star}$ )

\#25(lipid* near/3 emuls*)

\#26(lipid* near/3 powder*)

\#27(lipid* near/3 spread*)

\#28(lipid* near/3 paste*)

\#29(Nutributter* or Plumpy ${ }^{\star}$ )

\#30(LNS or iLiNS)

\#31\{or \#19-\#30\}

\#32\#18 or \#31

\#33[mh ^Infant]

\#34infant* ${ }^{\star}$ or toddler* or baby or babies or child*

\#35\#33 or \#34

\#36\#32 and \#35 in Trials

\section{MEDLINE Ovid}

1 exp Lipids/

2 fatty acid\$.tw,kf.

3 Docosahexaenoic acid.tw,kf.

4 Eicosapentaenoic Acid\$.tw,kf.

5 PUFAS.tw,kf.

6 lipid.tw,kf.

7 (omega $3 \$$ or omega $6 \$$ ).tw,kf.

8 (soy\$ or peanut or groundnut or whey or sesame or cashew or chickpea or oil\$).tw,kf.

9 or/1-8

10 Dietary Supplements/

11 Food, fortified/

Preventive lipid-based nutrient supplements given with complementary foods to infants and young children 6 to 23 months of age for

Copyright (c) 2019 The Cochrane Collaboration. Published by John Wiley \& Sons, Ltd. 
12 ((diet\$ or food\$) adj3 (fortif\$ or enrich\$ or supplement\$)).tw,kf.

13 (complement\$ adj3 (food\$ or feed\$)).tw,kf.

14 "Ready to use".tw,kf.

15 (RUSF or RUTF).tw,kf.

16 "point of use".tw,kf.

17 (home\$ adj2 fortif\$).tw,kf.

18 or $/ 10-17$

199 and 18

20 (lipid\$ adj3 nutrient\$).tw,kf.

21 (lipid\$ adj3 supplement\$).tw,kf.

22 lipid based.tw,kf.

23 (lipid\$ adj3 fortif\$).tw,kf.

24 (lipid\$ adj3 enrich\$).tw,kf.

25 (lipid\$ adj2 emuls\$).tw,kf.

26 (lipid\$ adj2 formulation\$).tw,kf.

27 (lipid\$ adj3 powder\$).tw,kf.

28 (lipid adj3 spread\$).tw,kf.

29 (lipid\$ adj3 paste\$).tw,kf.

30 (Nutributter\$ or Plumpy\$).tw,kf.

31 (LBNS\$ or LNS\$1 or iLiNS).tw,kf.

32 or $/ 20-31$

3319 or 32

34 Infant/

35 (baby or babies or infant\$ or toddler\$ or child\$).tw.

3634 or 35

3733 and 36

38 exp animals/ not humans.sh.

3937 not 38

\section{MEDLINE In-Process and Other Non-Indexed Citations Ovid}

1 lipid based.tw,kf.

2 (lipid\$ adj3 nutrient\$).tw,kf.

3 (lipid\$ adj3 supplement\$).tw,kf.

4 (lipid\$ adj3 fortif\$).tw,kf.

5 (lipid\$ adj3 enrich\$).tw,kf.

6 (lipid\$ adj2 emuls\$).tw,kf.

7 (lipid\$ adj2 formulation\$).tw,kf.

8 (Lipid\$ adj3 powder\$).tw,kf.

9 (lipid adj3 spread\$).tw,kf.

10 (lipid\$ adj3 paste\$).tw,kf.

11 (Nutributter\$ or Plumpy\$).tw,kf.

12 (LBNS\$ or LNS\$1 or iLiNS).tw,kf.

13 or $/ 1-12$

14 ((diet\$ or food\$) adj3 (fortif\$ or enrich\$ or supplement\$)).tw,kf.

15 (complement\$ adj3 (food\$ or feed\$)).tw,kf.

16 (RUSF or RUTF).tw,kf.

17 "point of use".tw,kf.

18 (home adj2 fortif\$).tw,kf.

19 lipid\$.tw,kf.

20 fatty acid\$.tw,kf.

21 Docosahexaenoic acid\$.tw,kf.

22 Eicosapentaenoic Acid\$.tw,kf.

23 PUFA\$.tw,kf.

24 (omega $3 \$$ or omega $6 \$$ ).tw, kf.

25 (soy\$ or peanut or groundnut or whey or sesame or cashew or chickpea or oil\$).tw,kf.

26 or/14-18

27 or/19-25

2826 and 27

2913 or 28

30 (baby or babies or infant\$ or toddler\$ or child\$).tw.

3129 and 30

Preventive lipid-based nutrient supplements given with complementary foods to infants and young children 6 to 23 months of age for

Copyright (c) 2019 The Cochrane Collaboration. Published by John Wiley \& Sons, Ltd. 


\section{MEDLINE E-pub ahead of print Ovid}

1 lipid based.tw,kf.

2 (lipid\$ adj3 nutrient\$).tw,kf.

3 (lipid\$ adj3 supplement\$).tw,kf.

4 (lipid\$ adj3 fortif\$).tw,kf.

5 (lipid\$ adj3 enrich\$).tw,kf.

6 (lipid\$ adj2 emuls\$).tw,kf.

7 (lipid\$ adj2 formulation\$).tw,kf.

8 (Lipid\$ adj3 powder\$).tw,kf.

9 (lipid adj3 spread\$).tw,kf.

10 (lipid\$ adj3 paste\$).tw,kf.

11 (Nutributter\$ or Plumpy\$).tw,kf.

12 (LBNS\$ or LNS\$1 or iLiNS).tw,kf.

13 or/1-12

14 ((diet\$ or food\$) adj3 (fortif\$ or enrich\$ or supplement\$)).tw,kf.

15 (complement\$ adj3 (food\$ or feed\$)).tw,kf.

16 (RUSF or RUTF).tw,kf.

17 "point of use".tw,kf.

18 (home adj2 fortif\$).tw,kf.

19 lipid\$.tw,kf.

20 fatty acid\$.tw,kf.

21 Docosahexaenoic acid\$.tw, kf.

22 Eicosapentaenoic Acid\$.tw,kf.

23 PUFA\$.tw,kf.

24 (omega $3 \$$ or omega $6 \$$ ).tw, kf.

25 (soy\$ or peanut or groundnut or whey or sesame or cashew or chickpea or oil\$).tw,kf.

26 or/14-18

27 or $/ 19-25$

2826 and 27

2913 or 28

30 (baby or babies or infant\$ or toddler\$ or child\$).tw.

3129 and 30

\section{Embase Ovid}

1 exp Lipids/

2 fatty acid\$.tw,kw.

3 Docosahexaenoic acid.tw,kw.

4 Eicosapentaenoic Acid\$.tw,kw.

5 PUFA\$.tw,kw.

6 lipid\$.tw,kw.

7 (omega $3 \$$ or omega $6 \$$ ).tw,kw.

8 (soy\$ or peanut or groundnut or whey or sesame or cashew or chickpea or oil\$).tw,kw.

9 or $/ 1-8$

10 dietary supplement/

11 fortified food/

12 ((diet\$ or food\$) adj3 (fortif\$ or enrich\$ or supplement\$)).tw,kw.

13 (complement\$ adj3 (food\$ or feed\$)).tw,kw.

14 "Ready to use".tw,kw.

15 (RUSF or RUTF).tw,kw.

16 "point of use".tw,kw.

17 (home\$ adj2 fortif\$).tw,kw.

18 or $/ 10-17$

199 and 18

20 lipid based.tw,kw.

21 (lipid\$ adj3 nutrient\$).tw,kw.

22 (lipid\$ adj3 supplement\$).tw,kw.

23 (lipid\$ adj3 fortif\$).tw,kw.

24 (lipid\$ adj3 enrich\$).tw,kw.

25 (lipid\$ adj2 emuls\$).tw,kw.

26 (lipid\$ adj2 formulation\$).tw,kw.

27 (Lipid\$ adj3 powder\$).tw,kw.

Preventive lipid-based nutrient supplements given with complementary foods to infants and young children 6 to 23 months of age for

Copyright (c) 2019 The Cochrane Collaboration. Published by John Wiley \& Sons, Ltd. 
28 (lipid adj3 spread\$).tw,kw.

29 (lipid\$ adj3 paste\$).tw,kw.

30 (Nutributter\$ or Plumpy\$).tw,kw.

31 (LBNS\$ or LNS\$1 or iLiNS).tw,kw.

32 or/20-31

3319 or 32

34 infant/

35 (baby or babies or infant\$ or toddler\$ or child\$).tw.

3634 or 35

3733 and 36

38 exp animals/ or exp invertebrate/ or animal experiment/ or animal model/ or animal tissue/ or animal cell/ or nonhuman/

39 human/ or normal human/ or human cell/

4038 not 39

4137 not 40

\section{CINAHL Plus EBSCOhost (Cumulative Index to Nursing and Allied Health Literature}

S1(MH "Lipids+")

S2TI (lipid*) or AB (lipid*)

S3TI(Docosahexaenoic acid $\left.{ }^{\star}\right)$ OR AB(Docosahexaenoic acid ${ }^{\star}$ )

S4TI( Eicosapentaenoic acid*) OR AB( Eicosapentaenoic acid*)

S5TI(PUFA*) OR AB(PUFA* $)$

S6TI(omega $3^{\star}$ or omega $6^{\star}$ ) OR AB (omega $3^{\star}$ or omega $6^{\star}$ )

$\mathrm{S} 7 \mathrm{TI}$ (soy* or peanut or groundnut or whey or sesame or cashew or chickpea or oil ${ }^{\star}$ ) or AB(soy* or peanut or groundnut or whey or sesame or cashew or chickpea or oil $\left.{ }^{\star}\right)$

S8TI(fatty acid*) OR AB (fatty acid* $)$

S9S1 OR S2 OR S3 OR S4 OR S5 OR S6 OR S7 OR S8

S10(MH "Dietary Supplements")

S11(MH "Dietary Supplementation")

S12(MH "Food, Fortified")

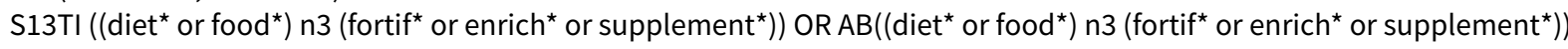

S14TI (complement ${ }^{\star}$ N3 $\left(\right.$ food $^{*}$ or feed $\left.{ }^{\star}\right)$ ) or AB (complement ${ }^{\star}$ N3 $\left(\right.$ food $^{\star}$ or feed $\left.^{\star}\right)$ )

S15"Ready to use"

S16(RUSF or RUTF)

S17"point of use"

S18TI (home* N2 fortif*) OR AB(home* N2 fortif*)

S19S10 OR S11 OR S12 OR S13 OR S14 OR S15 OR S16 OR S17 OR S18

S20S9 AND S19

S21TI (lipid based) or AB (lipid based)

S22TI(lipid* N3 supplement ${ }^{\star}$ ) OR AB( lipid* N3 supplement ${ }^{\star}$ )

S23TI(lipid ${ }^{\star}$ N3 nutrient $\left.{ }^{\star}\right)$ OR AB (lipid ${ }^{\star}$ N3 nutrient ${ }^{\star}$ )

S24TI(lipid ${ }^{\star}$ N3 fortif*) OR AB (lipid* N3 fortif*)

S25TI(lipid* N3 formulation*) OR AB (lipid* N3 formulation*)

S26TI(lipid* N3 enrich*) OR AB(lipid* N3 enrich*)

S27TI(lipid* N3 emuls*) OR AB(lipid* N3 emuls*)

S28TI(lipid* N3 powder*) OR AB(lipid* N3 powder*)

S29TI(lipid N3 spread*) OR AB(lipid N3 spread*)

S30TI(lipid* N3 paste*) OR AB(lipid* N3 paste*)

S31Nutributter*

S32 Plumpy*

S33TI(LNS 1 or iLiNS) OR AB( LNS 1 or iLiNS)

S34S21 OR S22 OR S23 OR S24 OR S25 OR S26 OR S27 OR S28 OR S29 OR S 30 OR S 31 OR S 32 OR S 33

S35S20 OR S34

S36(MH "Infant")

S37TI(baby or babies or infant* or toddler $^{\star}$ or child ${ }^{\star}$ ) OR AB (baby or babies or infant* or toddler or child $^{\star}$ )

S38S36 OR S37

S39S35 AND S38

\section{Science Citation Index (SCI) and Social Sciences Citation Index (SSCI) Web of Science}

\#5 \#4 AND \#3

\#4 TS=(infant ${ }^{\star}$ OR child $^{\star}$ OR toddler ${ }^{\star}$ or baby or babies)

\#3 \#2 OR \#1

Preventive lipid-based nutrient supplements given with complementary foods to infants and young children 6 to 23 months of age for

Copyright (c) 2019 The Cochrane Collaboration. Published by John Wiley \& Sons, Ltd. 
\#2 TS=(Nutributter* OR Plumpy* OR LNS OR iLiNS OR "lipid based" )

\#1 TS=(( "fatty acid*" OR PUFA OR PUFAs OR "omega $3^{\star " ~ O R ~ " o m e g a ~} 6 *$ " OR soy* OR peanut* OR groundnut* OR whey* OR sesame* OR

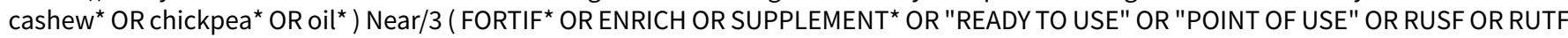
OR PASTE* OR SPREAD* OR FORMULAT* OR EMULS* OR NUTRIENT* OR POWDER $\left.{ }^{\star}\right)$ )

\section{Conference Proceedings Citation Index - Science (CPCI-S) and Conference Proceedings Citation Index - Social Science \& Humanities (CPCI-SS\&H) Web of Science}

\section{\#5 \#4 AND \#3}

DocType=All document types; Language=All languages; \#4 TS=(infant* OR child* OR toddler* or baby or babies) DocType=All document types; Language=All languages; \#3 \#2 OR \#1

DocType=All document types; Language=All languages; \#2 TS=(Nutributter* OR Plumpy* OR LNS OR iLiNS OR "lipid based" )

DocType=All document types; Language=All languages;

\#1 TS=(( "fatty acid*" OR PUFA OR PUFAs OR "omega $3^{\star *}$ OR "omega 6 " OR soy* OR peanut* OR groundnut* OR whey* OR sesame* OR

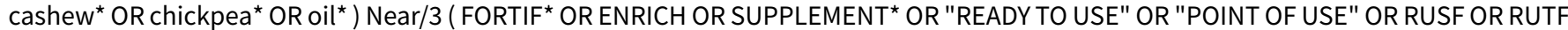
OR PASTE* OR SPREAD* OR FORMULAT* OR EMULS* OR NUTRIENT* OR POWDER $\left.{ }^{\star}\right)$ )

DocType=All document types; Language=All languages;

\section{Cochrane Database of Systematic Reviews (CDSR), part of the Cochrane Library}

$\# 1$ [mh Lipids]

\#3((Docosahexaenoic or Eicosapentaenoic) next acid $\left.{ }^{\star}\right): t i, a b$ \#4(PUFA or PUFAs):ti, ab

\#5lipid*:ti,ab 23529

\#6(omega next $\left(3^{\star}\right.$ or $\left.\left.6^{\star}\right)\right)$ :ti,ab

$\# 7\left(\right.$ soy $^{\star}$ or peanut or groundnut or whey or sesame or cashew or chickpea or oil $\left.{ }^{\star}\right): t i, a b$

\#8\{or \#1-\#7\}

\#9[mh "Dietary Supplements"]

\#10[mh "Food, fortified"]

$\# 11$ ((diet ${ }^{\star}$ or food $\left.{ }^{\star}\right)$ near/3 (fortif* or enrich* or supplement $\left.\left.{ }^{\star}\right)\right)$ :ti,ab

\#12(complement ${ }^{\star}$ near $/ 3$ (food ${ }^{\star}$ or feed $\left.^{\star}\right)$ ):ti,ab

\#13"Ready to use":ti,ab

\#14"point of use":ti,ab

\#15(RUSF or RUTF):ti,ab

\#16(home* near/2 fortif*):ti,ab

$\# 17$ or \#9-\#16\}

\#18\#8 and \#17

\#19(lipid next based):ti,ab

\#20(lipid* near/3 supplement ${ }^{\star}$ ):ti,ab

\#21(lipid* near/3 nutrient ${ }^{\star}$ ):ti,ab

\#22(lipid* near/3 fortif*):ti,ab

\#23(lipid* near/3 formulation*):ti,ab

\#24(lipid* near/3 enrich*):ti,ab

\#25(lipid* near/3 emuls*):ti,ab

\#26(lipid* near/3 powder $\left.{ }^{\star}\right): t i, a b$

\#27(lipid* near/3 spread*):ti,ab

\#28(lipid* near/3 paste*):ti,ab

\#29(Nutributter* or Plumpy*):ti,ab

\#30(LNS*1 or iLiNS):ti,ab

\#31\{or \#19-\#30\}

\#32\#18 or \#31

\#33[mh ^Infant]

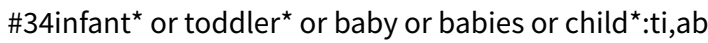

\#35\#33 or \#34

\#36\#32 and \#35 in Cochrane Reviews (Reviews and Protocols)

\section{Database of Abstracts of Reviews of Effect (DARE), part of the Cochrane Library}

$\# 1$ [mh Lipids]

\#3((Docosahexaenoic or Eicosapentaenoic) next acid*):ti,ab \#4(PUFA or PUFAs):ti,ab

Preventive lipid-based nutrient supplements given with complementary foods to infants and young children 6 to 23 months of age for health, nutrition, and developmental outcomes (Review)

Copyright (c) 2019 The Cochrane Collaboration. Published by John Wiley \& Sons, Ltd. 
\#5lipid*:ti,ab 23529

\#6(omega next $\left(3^{\star}\right.$ or $\left.\left.6^{\star}\right)\right)$ :ti,ab

$\# 7$ (soy* or peanut or groundnut or whey or sesame or cashew or chickpea or oil ${ }^{\star}$ ):ti,ab

\#8\{or \#1-\#7\}

\#9[mh "Dietary Supplements"]

$\# 10$ [mh "Food, fortified"]

$\# 11$ ((diet ${ }^{\star}$ or food $\left.{ }^{\star}\right)$ near/3 (fortif* or enrich* or supplement $\left.\left.{ }^{\star}\right)\right):$ ti,ab

\#12(complement* near/3 (food* or feed $\left.{ }^{\star}\right)$ ):ti,ab

\#13"Ready to use":ti,ab

\#14"point of use":ti,ab

\#15(RUSF or RUTF):ti,ab

\#16(home* near/2 fortif*):ti,ab

\#17\{0r \#9-\#16\}

\#18\#8 and \#17

\#19(lipid next based):ti,ab

\#20(lipid* near/3 supplement ${ }^{\star}$ ):ti,ab

\#21(lipid* near/3 nutrient $\left.{ }^{\star}\right)$ :ti,ab

\#22(lipid ${ }^{\star}$ near/3 fortif*):ti,ab

\#23(lipid* near/3 formulation ${ }^{\star}$ ):ti,ab

\#24(lipid* near/3 enrich*):ti,ab

\#25(lipid* near/3 emuls*):ti,ab

\#26(lipid ${ }^{\star}$ near/3 powder $\left.{ }^{\star}\right):$ ti,ab

\#27(lipid ${ }^{\star}$ near/3 spread*):ti,ab

\#28(lipid* near/3 paste*):ti,ab

\#29(Nutributter ${ }^{\star}$ or Plumpy*):ti,ab

\#30(LNS*1 or iLiNS):ti,ab

\#31\{or \#19-\#30\}

\#32\#18 or \#31

\#33[mh ^Infant]

\#34infant* or toddler* or baby or babies or child*:ti,ab

\#35\#33 or \#34

\#36\#32 and \#35 in Other Reviews

\section{Epistemonikos (epistemonikos.org)}

(title:(LIPID* OR FATTY ACID* OR OMEGA OR Docosahexaenoic OR Eicosapentaenoic OR soy* OR peanut OR groundnut OR whey OR sesame OR cashew OR chickpea OR oil*) OR abstract:(LIPID* OR FATTY ACID* OR OMEGA OR Docosahexaenoic OR Eicosapentaenoic OR soy* OR peanut OR groundnut OR whey OR sesame OR cashew OR chickpea OR oil*)) AND (title:(fortif* OR enrich* OR supplement* OR "Ready to use" OR "point of use" OR RUSF OR RUTF) OR abstract:(fortif* OR enrich* OR supplement* OR "Ready to use" OR "point of use" OR RUSF OR RUTF)) AND title:(babies OR children OR infant*)

\section{LIMITED TO}

PUBLICATION TYPE: SYSTEMATIC REVIEW

SYSTEMATIC REVIEW QUESTION : INTERVENTIONS

\section{POPLINE (www.popline.org)}

((ALL FIELDS(lipid* OR "fatty acid*" OR PUFA OR PUFAs OR "omega $3^{\star} "$ OR "omega 6 *" OR soy* OR peanut* OR groundnut* OR whey* OR sesame* OR cashew* OR chickpea* OR oil*) AND ALL FIELDS (FORTIF* OR ENRICH OR SUPPLEMENT* OR "READY TO USE" OR "POINT OF USE" OR RUSF OR RUTF OR PASTE* OR SPREAD* OR FORMULAT* OR EMULS* OR NUTRIENT* OR POWDER*)) OR (Nutributter* OR Plumpy* OR LNS OR iLiNS)) AND ALL FIELDS (infan* OR child* OR baby OR babies OR toddler*)

\section{ClinicalTrials.gov (clinicaltrials.gov)}

Interventional Studies | LIPID-BASED OR LNS OR iLINS OR "NUTRIENT SUPPLEMENT" OR PASTE OR SPREAD OR BLEND OR NUTRIBUTTER OR Plumpy OR PLUMPYNUT | Child

\section{World Health Organization International Clinical Trials Registry Platform (WHO ICTRP; who.int/trialsearch)}

(LIPID BASED OR LNS OR iLINS OR NUTRIENT SUPPLEMENT OR SPREAD OR paste OR BLEND OR NUTRIBUTTER OR Plumpy OR PLUMPYNUT) NOT (teeth OR oral heath OR dentistry)

\section{IBECS (Índice Bibliográfico Español en Ciencias de la Salud; ibecs.isciii.es)}

WORD| "lipid based" OR LNS OR iLiNS OR "nutrient supplement" OR paste OR spread OR blend OR nutributter OR Plumpy OR plumpynut

Preventive lipid-based nutrient supplements given with complementary foods to infants and young children 6 to 23 months of age for 
AND

WORD $\mid$ infan* OR child* OR baby OR babies OR toddler*

\section{SciELO (Scientific Electronic Library Online; www.scielo.br)}

"lipid based" OR LNS OR iLiNS OR "nutrient supplement" OR paste OR spread OR blend OR nutributter OR Plumpy OR plumpynut [All indexes]

AND

infan* OR child* OR baby OR babies OR toddler ${ }^{\star}$ [All indexes]

AIM (Africa Index Medicus; search.bvsalud.org/ghl/?lang=en\&submit=Search\&where=REGIONAL)

(lipid based OR LNS OR iLiNS OR nutrient supplement OR paste OR spread OR blend OR nutributter OR Plumpy OR plumpynut) AND (infan OR child OR baby OR babies OR toddler)

IMEMR (Index Medicus for the Eastern Mediterranean Region; search.bvsalud.org/ghl/? lang=en\&submit=Search\&where=REGIONAL)

(lipid based OR LNS OR iLiNS OR nutrient supplement OR paste OR spread OR blend OR nutributter OR Plumpy OR plumpynut) [Title]

AND

(infan OR child OR baby OR babies OR toddler) [Title]

LILACS (Latin American and Caribbean Health Sciences Literature; lilacs.bvsalud.org/en)

(tw:("lipid based" OR LNS OR iLiNS OR "nutrient supplement" OR paste OR spread OR blend OR nutributter OR Plumpy OR plumpynut) AND (tw:(infan* OR child* OR baby OR babies OR toddler*)

\section{PAHO/WHO Institutional Repository for Information Sharing (iris.paho.org/xmlui)}

("lipid based" OR LNS OR iLiNS OR "nutrient supplement" OR paste OR spread OR blend OR nutributter OR Plumpy OR plumpynut) AND (infan* OR child* OR baby OR babies OR toddler*)

WHOLIS (WHO Library Database; search.bvsalud.org/ghl/?lang=en\&submit=Search\&where=REGIONAL)

words or phrase "lipid based" OR LNS OR iLiNS OR "nutrient supplement" OR paste OR spread OR blend OR nutributter OR Plumpy OR plumpynut AND words or phrase infan* OR child* OR baby OR babies OR toddler*

WPRIM (Western Pacific Index Medicus; search.bvsalud.org/ghl/?lang=en\&submit=Search\&where=REGIONAL)

("lipid based" OR LNS OR iLiNS OR "nutrient supplement" OR paste OR spread OR blend OR nutributter OR Plumpy OR plumpynut) AND (infan* OR child* OR baby OR babies OR toddler*)

IMSEAR (Index Medicus for the South-East Asian Region; search.bvsalud.org/ghl/?lang=en\&submit=Search\&where=REGIONAL)

("lipid based" OR LNS OR iLiNS OR "nutrient supplement" OR paste OR spread OR blend OR nutributter OR Plumpy OR plumpynut) [Title] AND (infan* OR child* OR baby OR babies OR toddler*) [Title]

IndMED (indmed.nic.in/indmed.html)

(lipid based OR LNS OR iLiNS OR nutrient supplement OR paste OR spread OR blend OR nutributter OR Plumpy OR plumpynut) AND (infan OR child OR baby OR babies OR toddler)

\section{Native Health Research Database (hscssl.unm.edu/nhd)}

Keywords: (Supplement AND child)

\section{Appendix 3. Extraction sheet}

\section{Author:}

Year of publication:

Preventive lipid-based nutrient supplements given with complementary foods to infants and young children 6 to 23 months of age for 
(Continued)

Journal:

\section{Study funded by:}

\section{Country:}

\section{Study design:}

\section{Setting (Hospital/Outpatient/Community):}

\section{Setting (Stable/Emergency):}

\section{Target population:}

\begin{tabular}{|c|c|c|}
\hline \multirow[t]{4}{*}{ Baseline Indicators } & \multicolumn{2}{|l|}{ Anemia: } \\
\hline & \multicolumn{2}{|l|}{ BMI: } \\
\hline & \multicolumn{2}{|l|}{ MUAC: } \\
\hline & \multicolumn{2}{|l|}{ Other: } \\
\hline \multirow{10}{*}{$\begin{array}{l}\text { Intervention/Compari- } \\
\text { son Groups }\end{array}$} & \multicolumn{2}{|l|}{ Intervention/Comparison: } \\
\hline & \multicolumn{2}{|c|}{ Supplementary food composition/Nutrient density: } \\
\hline & \multicolumn{2}{|l|}{ Produced by: } \\
\hline & \multicolumn{2}{|l|}{ Dose/Frequency: } \\
\hline & \multicolumn{2}{|l|}{ Duration of intervention: } \\
\hline & \multicolumn{2}{|l|}{ Period of intervention: } \\
\hline & \multicolumn{2}{|l|}{ Intervention given through: } \\
\hline & \multicolumn{2}{|l|}{ Composition of LNS/Nutrient density: } \\
\hline & \multicolumn{2}{|l|}{ Duration of follow-up: } \\
\hline & \multicolumn{2}{|l|}{ Number of participants: } \\
\hline \multirow[t]{7}{*}{ Risk of Bias } & \multirow{2}{*}{$\begin{array}{l}\text { Randomization/Sequence genera- } \\
\text { tion }\end{array}$} & Author judgment for risk of bias: Low, High or Unclear \\
\hline & & Quote from the paper: \\
\hline & \multirow[t]{2}{*}{ Allocation concealment } & Author judgment for risk of bias: Low, High or Unclear \\
\hline & & Quote from the paper: \\
\hline & \multirow[t]{2}{*}{ Assessment blinding - Outcome } & Author judgment for risk of bias: Low, High or Unclear \\
\hline & & Quote from the paper: \\
\hline & Blinding of participants/personnel & Author judgment for risk of bias: Low, High or Unclear \\
\hline
\end{tabular}


Quote from the paper:

\begin{tabular}{ll}
\hline Selective reporting & Author judgment for risk of bias: Low, High or Unclear \\
\cline { 2 - 2 } & Quote from the paper: \\
\hline Attribution/ Loss to follow up & Author judgment for risk of bias: Low, High or Unclear \\
\cline { 2 - 2 } & Quote from the paper: \\
\hline Other bias & Author judgment for risk of bias: Low, High or Unclear \\
\cline { 2 - 2 } & Quote from the paper: \\
\hline Orimary/Secondary: & \\
\hline Units: & \\
\hline Numbers (for all groups) & \\
\hline
\end{tabular}

\section{Limitations:}

Comments:

\section{Footnotes}

BMI: Body mass index.

LNS: Lipid-based nutrient supplements.

MUAC: Mid-upper arm circumference.

\section{Appendix 4. Criteria for assessing risk of bias in RCTs}

\section{Random sequence generation (checking for possible selection bias)}

We assessed whether the method used to generate the allocation sequence was described in sufficient detail to allow an assessment of whether it produced comparable groups.

1. Low risk of bias: any truly random process (for example, random number table, computer random number generator)

2. High risk of bias: any non-random process (for example, odd or even date of birth, hospital or clinic record number)

3. Unclear risk of bias: insufficient information provided to permit judgement of high or low risk of bias

\section{Allocation concealment (checking for possible selection bias)}

We assessed whether the method used to conceal the allocation sequence was described in sufficient detail to determine whether intervention allocations could have been foreseen in advance of, or during, enrolment.

1. Low risk of bias: telephone or central randomisation; consecutively numbered, sealed, opaque envelopes

2. High risk of bias: open random allocation, unsealed or non-opaque envelopes

3. Unclear risk of bias: insufficient information provided to permit judgement of high or low risk of bias

\section{Blinding of participants and personnel (checking for possible performance bias)}

We described all measures used, if any, to blind study participants and personnel from knowledge of which intervention a participant received.

We assessed the risk of performance bias associated with blinding of participants and personnel as follows. 
1. Low risk of bias: blinding of participants and personnel and unlikely that the blinding could have been broken, or no blinding or incomplete blinding but outcome unlikely to have been influenced

2. High risk of bias: participants and personnel not blinded, incomplete or broken blinding and outcome likely to have been influenced

3. Unclear risk of bias: insufficient information provided to permit judgement of high or low risk of bias

Whilst assessed separately, we combined the results into a single evaluation of risk of bias associated with blinding (Higgins 2011 a).

\section{Blinding of outcome assessment (checking for possible detection bias)}

We described all measures used, if any, to blind outcome assessors from knowledge of which intervention a participant received.

1. Low risk of bias: blinding of outcome assessment and unlikely that the blinding could have been broken, or no blinding but measurement unlikely to have been influenced

2. High risk of bias: no blinding of outcome assessment, measurement likely to have been influenced by lack of blinding, or blinding could have been broken

3. Unclear risk of bias: insufficient information provided to permit judgement of high or low risk of bias

\section{Incomplete outcome data (checking for possible attrition bias through withdrawals, dropouts, protocol deviations)}

We assessed the outcomes in each included study as follows.

1. Low risk of bias: no missing outcome data or missing outcome data were unlikely to bias the results based on the following considerations; study authors provided transparent documentation of participant flow throughout the study, the proportion of missing data was similar in the intervention and control groups, the reasons for missing data were provided and balanced across intervention and control groups, or the reasons for missing data were not likely to bias the results (for example, moving house)

2. High risk of bias: missing outcome data were likely to bias the result, 'as-treated (per protocol)' analysis was performed with substantial differences between the intervention received and that assigned at randomisation, or potentially inappropriate methods for imputation were used

3. Unclear risk of bias: insufficient information provided to permit judgement of high or low risk of bias

\section{Selective outcome reporting (checking for possible reporting bias)}

Selective reporting can lead to reporting bias. We compared the methods to the results and looked for outcomes that were measured (or likely to have been measured) but not reported.

1. Low risk of bias: all of the study's pre-specified outcomes and expected outcomes of interest to the review were reported

2. High risk of bias: not all of the study's pre-specified outcomes were reported, one or more reported primary outcomes were not prespecified, outcomes of interest were reported incompletely and so could not be used, or the study failed to include the results of a key outcome that was expected to be reported

3. Unclear risk of bias: insufficient information provided to permit judgement of high or low risk of bias

Other sources of bias (checking for other possible sources of bias not covered by the domains above)

We assessed if the study was free of other potential bias as follows.

1. Low risk of bias: similarity between outcome measures at baseline, similarity between potential confounding variables at baseline, or adequate protection of study arms against contamination

2. High risk of bias: no similarity between outcome measures at baseline, no similarity between potential confounding variables at baseline, or inadequate protection of study arms against contamination

3. Unclear risk of bias: insufficient information provided to permit judgement of high or low risk of bias

\section{WHAT'S NEW}

\begin{tabular}{lll}
\hline Date & Event & Description \\
\hline 13 May 2019 & Amended & Some typos corrected in the results. No change to conclusions. \\
\hline
\end{tabular}

\section{H I S T O R Y}

Protocol first published: Issue 3, 2017

Preventive lipid-based nutrient supplements given with complementary foods to infants and young children 6 to 23 months of age for 


\begin{tabular}{lll}
\hline Date & Event & Description \\
\hline 27 March 2019 & Amended & $\begin{array}{l}\text { Major change to protocol. Due to the identification of a substan- } \\
\text { tial number of RCTs and quasi-RCTs at review stage, the decision } \\
\text { was taken to restrict the inclusion criteria to these types of stud- } \\
\text { ies. }\end{array}$ \\
\hline
\end{tabular}

\section{CONTRIBUTIONS OF AUTHORS}

All review authors contributed to the development of the review.

Rehana A Salam (RAS), Yousaf Bashir, Sana Sadiq Sheikh and Afsah Zulfiqar Bhutta selected which studies to include, obtained copies of the studies and extracted data from the studies.

Jai K Das (JKD) and RAS entered data into RevMan, carried out the analysis and interpreted the results.

JKD, RAS, Zita Weise Prinzo and Zulfiqar A Bhutta (ZAB) drafted the final review.

As the contact author, ZAB has overall responsibility for the review.

\section{DECLARATIONS OF INTEREST}

We certify that we have no affiliations with, or involvement in, any organisation or entity with a direct financial interest in the subject matter of the review (e.g. employment, consultancy, stock ownership, honoraria, expert testimony).

Jai K Das - none known.

Rehana A Salam - none known.

Yousaf Bashir Hadi - none known.

Sana Sadiq Sheikh - none known.

Afsah Zulfiqar Bhutta - none known.

Zita Weise Prinzo is a full-time member of staff of the WHO.

Zulfiqar A Bhutta's institution was awarded a grant from the WHO to undertake this review.

The review authors alone are responsible for the views expressed in this publication; the views do not necessarily represent the official position, decisions, policy or views of the WHO.

\section{SOURCES OF SUPPORT}

\section{Internal sources}

- Evidence and Programme Guidance, Department of Nutrition for Health and Development, World Health Organization (WHO), Switzerland.

Zita Weise Prinzo is a full-time member of staff at the WHO

- Aga Khan University, Karachi, Pakistan.

Jai K Das, Rehana A Salam, Sana Sadiq Sheikh and Zulfiqar A Bhutta are full-time employees of Aga Khan University, Karachi

\section{External sources}

- The Bill \& Melinda Gates Foundation, USA.

WHO thanks the Bill \& Melinda Gates Foundation for supporting the preparation of systematic reviews of the evidence of the effects and harms of nutrition and nutrition-sensitive interventions.

- Evidence and Programme Guidance, Department of Nutrition for Health and Development, WHO, Switzerland.

WHO provided financial support for this work. 
- Nutrition International, Canada.

WHO thank Nutrition International for supporting the Evidence and Programme Guidance Unit, Department of Nutrition for Health and Development, for the preparation of systematic reviews of the evidence of the effects and harms of nutrition and nutrition-sensitive interventions.

\section{DIFFERENCES BETWEEN PROTOCOLANDREVIEW}

\section{Subgroup analysis and investigation of heterogeneity}

In our protocol (Das 2017), we had planned to conduct an analysis by 'duration of intervention' according to the following subgroups: 'less than three months versus three to six months versus six months or more'. However, in the review, we changed these subgroups to 'up to 6 months versus 6-12 months versus more than 12 months', according to the findings of the included studies and to be more clinically relevant.

We had also planned to conduct an analysis by 'age at follow-up' according to ' 6 to 11 months versus 12 to 23 months versus other' (Das 2017), but changed these in the review to the following subgroups: 'at 12 months versus at 18 months versus at 24 months versus at 36 months'. Again, this was done according to the findings of the included studies identified at the review stage, and to be more clinically relevant.

We could not conduct the following, preplanned subgroup analyses (Das 2017), since the included studies did not report this information.

1. Breastfeeding practices (breastfed versus not breastfed)

2. Frequency of intervention (daily versus weekly versus flexible)

3. Living in an emergency-affected country (Wisner 2002) or in a refugee or internally displaced persons' camp (yes versus no)

4. Anaemic status of participants at start of intervention (anaemic (defined as haemoglobin values $<110 \mathrm{~g} / \mathrm{L}$ ) versus non anaemic or unknown status)

These exploratory analyses have been archived in Table 1 for use in future updates of this review.

\section{Search methods}

We searched the Database of Abstracts of Reviews of Effects (DARE) to find other reviews.

\section{Inclusion criteria: Type of study}

In our protocol, we had specified that we would be including randomised controlled trials (RCTs), quasi-RCTs, controlled before-and-after studies (CBAs), and interrupted time series (ITS); however since almost all the included studies in the review were RCTs, we decided to restrict our inclusion to RCTs and quasi-RCTs only, to strengthen the quality of the evidence.

\section{INDEX TERMS}

\section{Medical Subject Headings (MeSH)}

*Infant Nutritional Physiological Phenomena; *Nutritional Status; Child Development [ ${ }^{*}$ physiology]; Dietary Fats [ ${ }^{*}$ administration \& dosage]; Dietary Supplements; Food, Formulated; Food, Fortified

\section{MeSH check words}

Child, Preschool; Humans; Infant 